\title{
Modular Approach to the Synthesis of 2-D Angular fused acenes
}

\author{
Mikhail Feofanov ${ }^{\dagger}$, Vladimir Akhmetov ${ }^{\dagger}$, Dmitry Sharapa ${ }^{\ddagger}$ and Konstantin Amsharov* ${ }^{\star}$, \\ † Friedrich-Alexander University Erlangen-Nuernberg, Department of Chemistry and Pharmacy, Organic \\ Chemistry II, \\ Nikolaus-Fiebiger Str. 10, 91058 Erlangen, Germany. \\ ‡ Institut für Katalyseforschung und -technologie, Karlsruhe Institute of Technology, Hermann-von-Helmholtz- \\ Platz 1, 76344 Eggenstein-Leopoldshafen, Germany. \\ $\S$ Institute of Chemistry, Organic Chemistry, Martin-Luther-University Halle-Wittenberg, Kurt-Mothes-Strasse \\ 2, D-o612o Halle, Germany
}

\section{Table of contents}

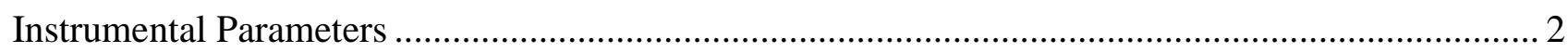

Possible PAHs through combination of building blocks............................................................. 4

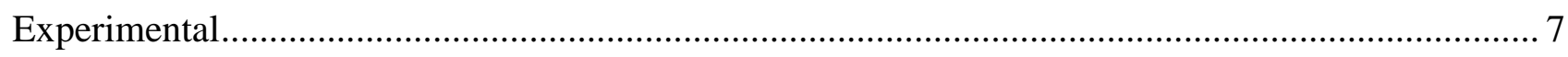

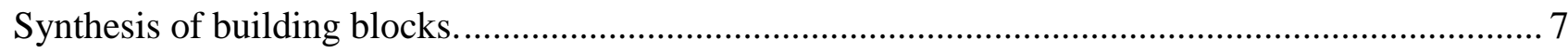

Synthesis of precursors P1-P9 for DPEX reaction. ........................................................... 12

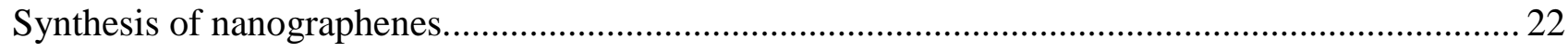

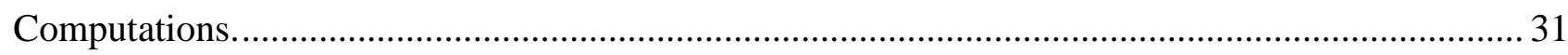

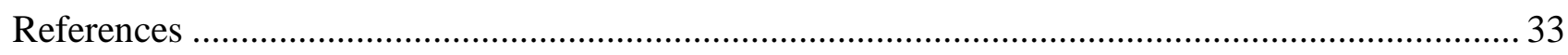

Spectral appendix $\left({ }^{1} \mathrm{H},{ }^{13} \mathrm{C}\right.$ NMR, LDI, HRMS, UV, Fluo, HPLC $)$.......................................... 35

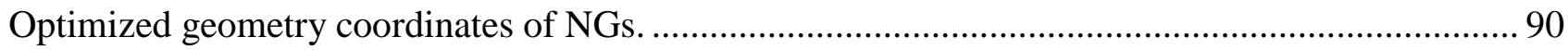




\section{Instrumental Parameters}

All chemicals and solvents were purchased in reagent grade from commercial suppliers (Acros $₫$, Sigma-Aldrich ${ }^{\circledR}$ or Fluka ${ }^{\circledR}$, Fluorochem ${ }^{\circledR}$, Merck ${ }^{\circledR}$, ChemPur $\left.{ }^{\circledR}\right)$ and used as received, unless otherwise specified. Solvents in HPLC grade were purchased from VWR® and Sigma-Aldrich ${ }^{\circledR}$. Flash column chromatography was performed on a Interchim PuriFlash XS420 using flash grade silica gel from (Machery-Nagel 60 M (40-63 mm, deactivated)).

NMR spectra were recorded on a Bruker Avance 400 at $400 \mathrm{MHz}\left({ }^{1} \mathrm{H}\right.$ NMR) and $100 \mathrm{MHz}\left({ }^{13} \mathrm{C}\right.$ NMR), Bruker Avance 300 operating at $300 \mathrm{MHz}$ ( ${ }^{1} \mathrm{H}$ NMR), a Bruker Avance Neo 500, operating at $500 \mathrm{MHz}\left({ }^{1} \mathrm{H}\right.$ NMR) at $120{ }^{\circ} \mathrm{C}$ and a Bruker Avance Neo 600, operating at $600 \mathrm{MHz}\left({ }^{1} \mathrm{H} \mathrm{NMR}\right)$ and $150 \mathrm{MHz}\left({ }^{13} \mathrm{C} \mathrm{NMR}\right)$ at room temperature. The signals were referenced to residual solvent peaks (in parts per million (ppm) ${ }^{1} \mathrm{H}$ : $\mathrm{CDCl}_{3}, 7.27$ ppm; $\mathrm{CD}_{2} \mathrm{Cl}_{2}, 5.32$ ppm; $\mathrm{C}_{6} \mathrm{D}_{6}, 7.16$ ppm; o-DCB-d ${ }^{4}, 6.93$ ppm; $\mathrm{C}_{2} \mathrm{D}_{2} \mathrm{Cl}_{4}, 6.00$ ppm; DMSO-d ${ }^{6}, 2.50$ ppm; Acetone- $\mathrm{d}_{6}, 2.05$ ppm; ${ }^{13} \mathrm{C}: \mathrm{CDCl}_{3}, 77.0 \mathrm{ppm}$; $\mathrm{C}_{2} \mathrm{D}_{2} \mathrm{Cl}_{4}, 73.8 \mathrm{ppm} ; \mathrm{CD}_{2} \mathrm{Cl}_{2}, 53.84 \mathrm{ppm}$; acetone- $\mathrm{d}_{6}, 29.9 \mathrm{ppm}$ ). Coupling constants were assigned as observed. The obtained spectra were evaluated with the program MestReNova.

(MA)LDI-MS spectra were recorded on a Shimadzu Biotech AXIMA Confidence MALDI-TOF.

High resolution APPI spectra were recorded on a Bruker ESI TOF maXis $4 \mathrm{G}$ instrument. The data was evaluated with the program Bruker Compass DataAnalysis 4.2.

HPLC measurements were performed on a Shimadzu Prominence Liquid Chromatograph LC-20AT with communication bus module CBM-20A, diode array detector SPDM20A, the degassing unit DGU-20A5 R, column oven CTO-20AC or CTO-20A, respectively and with auto sampler SIL-20A HT. For separation a Cosmosil 5-PBR column $(4.6 \mathrm{~mm}$ x $250 \mathrm{~mm})$ from Nacalai Tesque was used. As eluent a toluene/MeOH/ or toluene/1,2-dichlorobenzene mixture or pure toluene or 1,2dichlorobenzene was used (UV/Vis detection). The data was evaluated with the programs Shimadzu LCsolution and Shimadzu LabSolutions.

Fluorescence spectra were recorded on a Shimadzu RF-5301PC spectrofuorophotometer at rt.

Electrochemical data were obtained in o-dichlorobenzene solution of $\mathrm{Bu}_{4} \mathrm{NPF}_{6}(0.1 \mathrm{M})$. Cyclic voltammagrams were obtained using a glassy carbon working electrode, a Pt counter electrode, and a non-aqueous silver $\left(0.01 \mathrm{M} \mathrm{AgNO}_{3}, 0.1 \mathrm{TBAP}\right.$ in acetonitrile) reference electrode. The data were evaluated with the programs Gamry Echem Analyst v.7.07. 
TLC analyses were carried out with TLC sheets coated with silica gel with fluorescent indicator 254 nm from Machery-Nagel (ALUGRAM® SIL G/UV254) and visualized via UV-light of 254nm or 366 nm. 


\section{Possible PAHs through combination of building blocks}

Table 1. Theoretically possible PAHs which can be obtained by combination of 10 DHA and 2 FBA

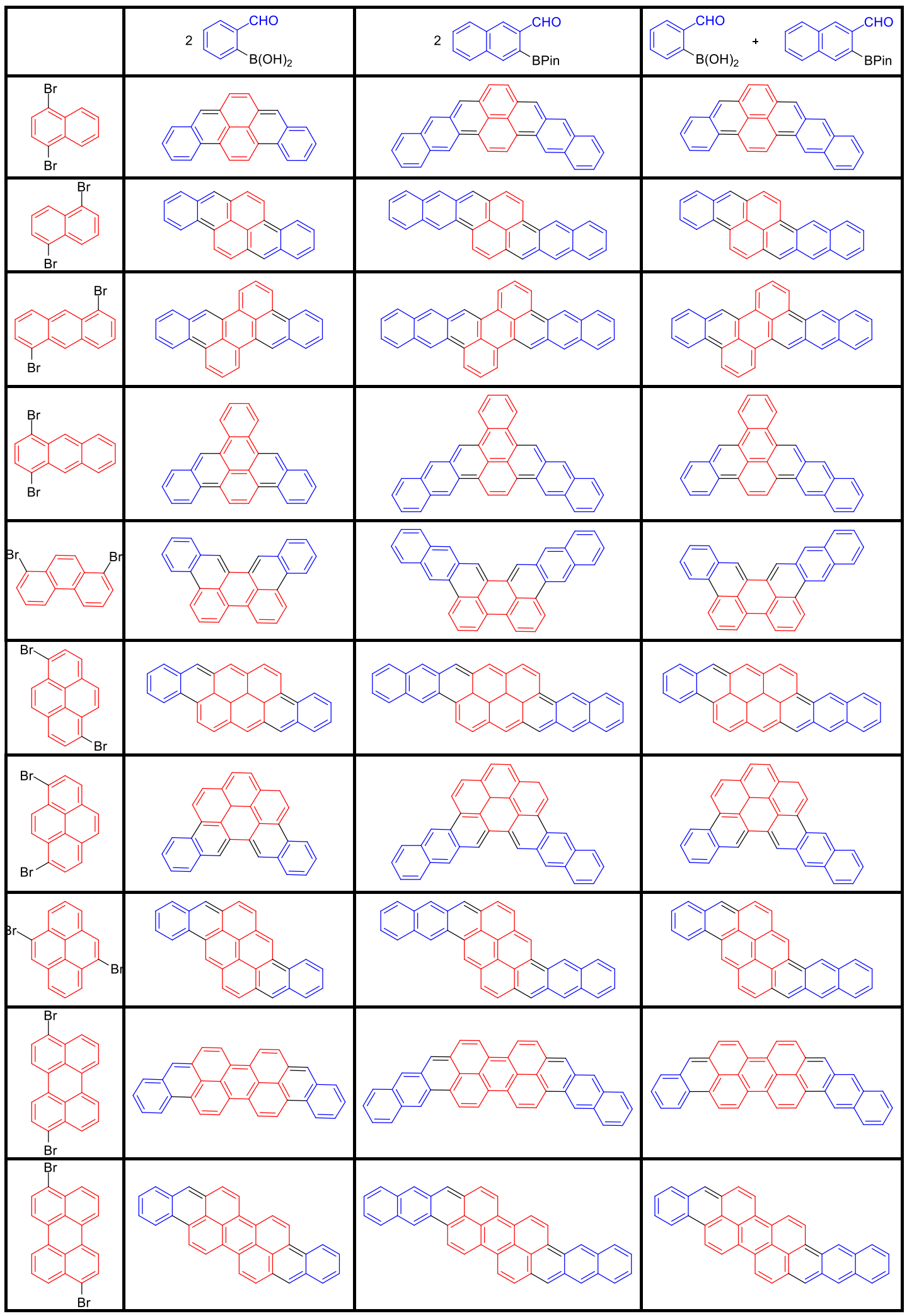


Table 2. Theoretically possible PAHs which can be obtained by combination of 1010 DHA, 2 FBA and 2 BS.

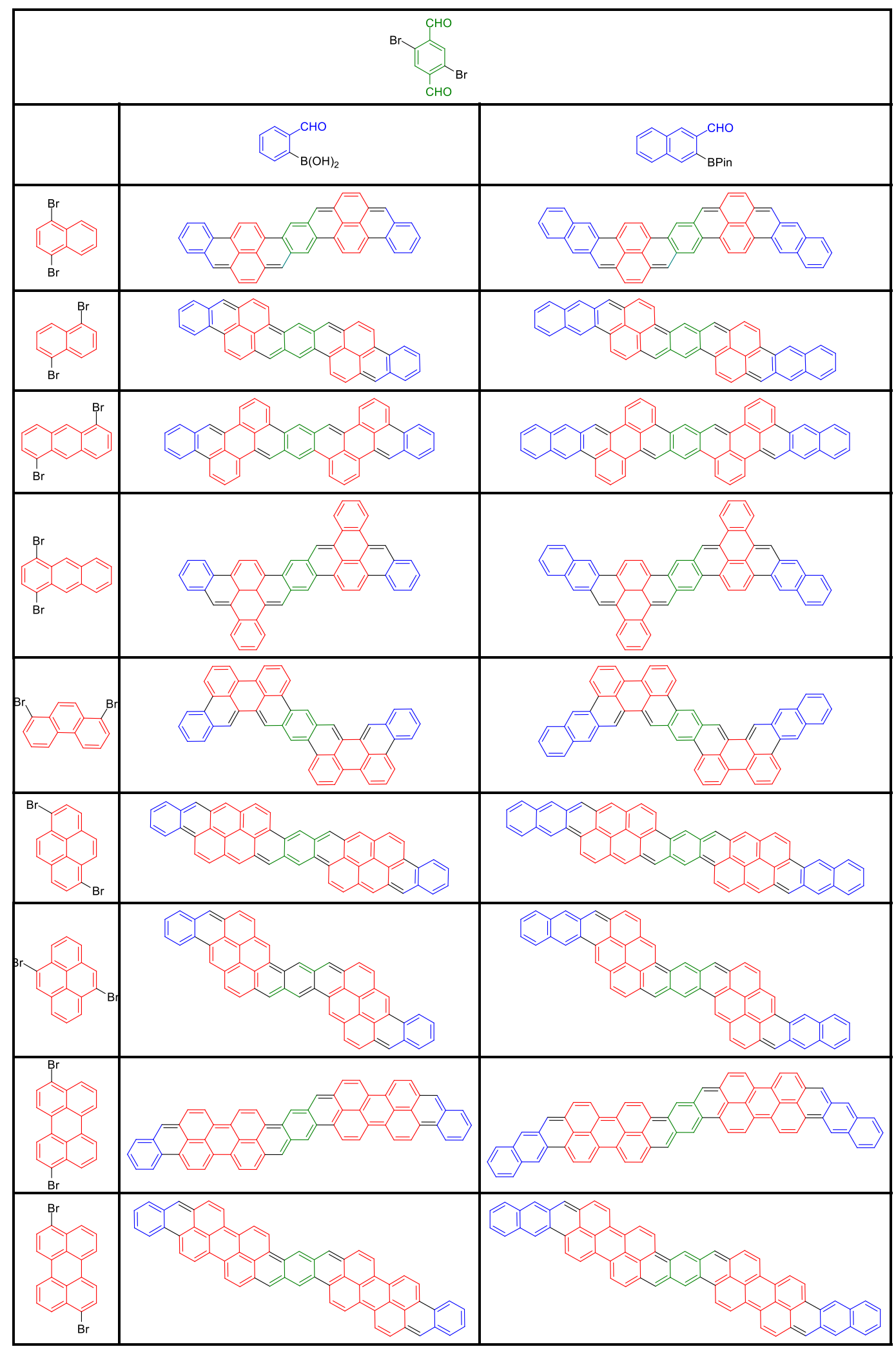




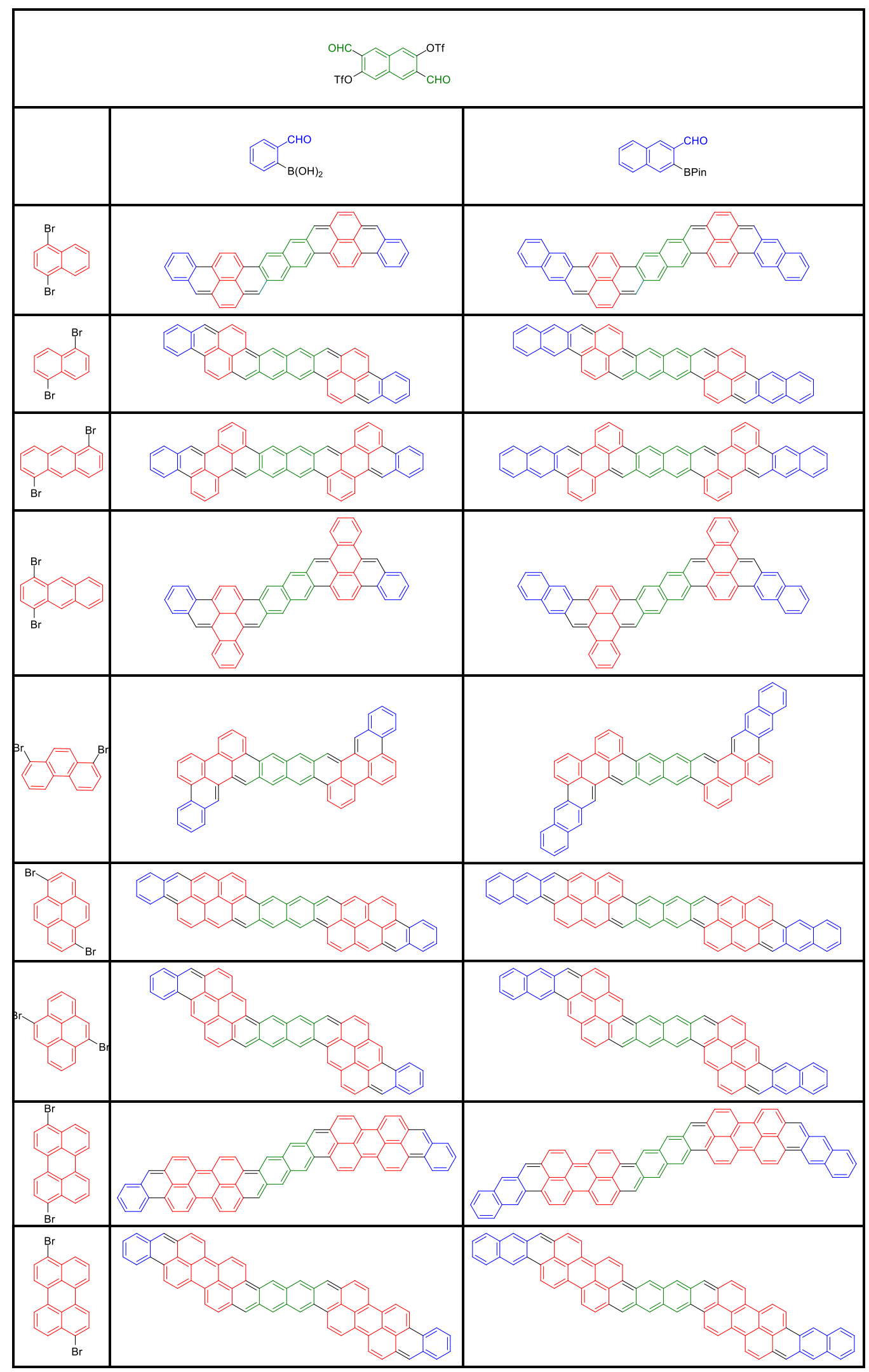




\section{Experimental}

\section{Synthesis of building blocks.}<smiles>CC(C)c1ccc2c(c1)C(=O)c1c(N)ccc(N)c1C2=O</smiles>

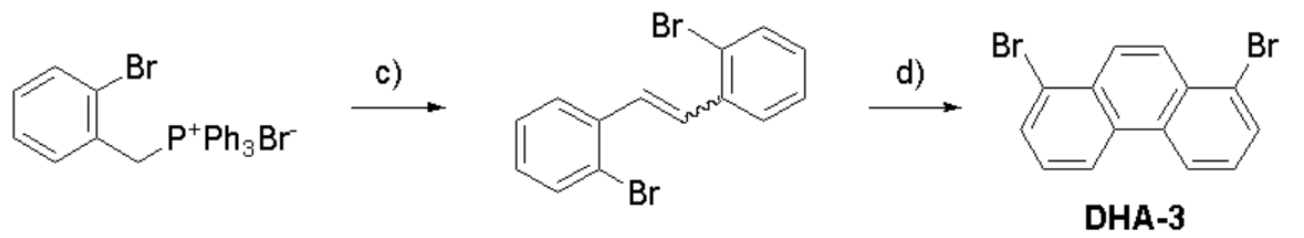<smiles>c1cc2c3c(ccc4c3c1CCC4)CCC2</smiles>

e)<smiles>Brc1cc2c3c(c(Br)cc4c3c1CCC4)CCC2</smiles><smiles>Brc1cc2cccc3c(Br)cc4cccc1c4c23</smiles>

DHA-4<smiles>Cc1cccc2c(N)cccc12</smiles><smiles>Cc1cc(Br)c(C)cc1Br</smiles>

BS-1

Figure 1. Synthesis of DHA-1, DHA-2, DGA-3, DGA-4, DGA-5, DGA-5a, BS-1. a) 1. $t$-BuONO, $\mathrm{CuBr}_{2}, \mathrm{MeCN}$. 2. $\mathrm{NaBH}_{4}, i$-PrOH. 3. $\mathrm{SnCl}_{2}, \mathrm{AcOH} ; 35 \%$. b) 1. $t$-BuONO, $\mathrm{CuBr}_{2}, \mathrm{MeCN}$. 2) $\mathrm{NaBH}_{4}$, $i$-PrOH. 3) $\mathrm{SnCl}_{2}, \mathrm{AcOH} ; 23 \%$. c) 2-bromobenzaldehyde, KOt-Bu, THF; 77\%. d) $h v, \mathrm{I}_{2}$, cyclohexane; 48\%. e) $\mathrm{Br}_{2}, \mathrm{DCM} ; 35 \%$. f) DDQ, toluene; 44\%. g) $\mathrm{NaNO}_{2}, \mathrm{H}_{2} \mathrm{SO}_{4}, \mathrm{CuBr}, \mathrm{HBr} ; 15 \%$. h) $\mathrm{NaNO}_{2}$, $\mathrm{H}_{2} \mathrm{SO}_{4}, \mathrm{KI} ; 47 \%$. i) $1 . \mathrm{H}_{2} \mathrm{SO}_{4}, \mathrm{CrO}_{3}, \mathrm{CH}_{3} \mathrm{COOH},\left(\mathrm{CH}_{3} \mathrm{CO}\right)_{2} \mathrm{O} .2 . \mathrm{H}_{2} \mathrm{O}, \mathrm{EtOH}, \mathrm{H}_{2} \mathrm{SO}_{4} ; 48 \%$. 
FBA-2 ${ }^{1}$, DHA-3 ${ }^{2}$, DHA-5a ${ }^{1}$ were synthesized according to literature methods.

\section{1,4-dibromoanthracene (DHA-1 $)^{3}$.}

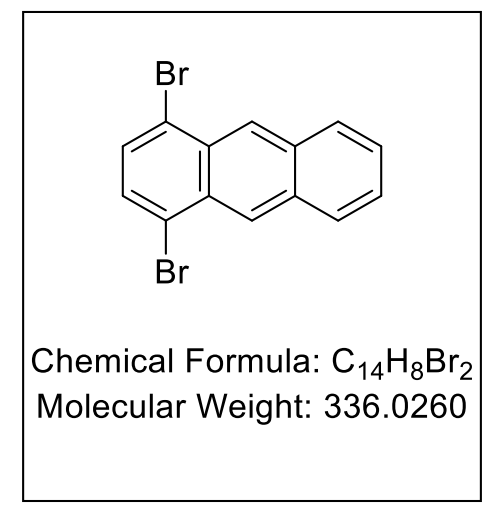

A mixture of 1,4-diaminoanthraquinone (2.01 g, $8.39 \mathrm{mmol})$, copper(II) bromide $(4.22 \mathrm{~g}, 18.8 \mathrm{mmol})$ and acetonitrile $(16 \mathrm{~mL})$ in a $100 \mathrm{~mL}$ two-necked round bottom flask was stirred with a stir for a short while at room temperature. Then the reaction temperature was increased up to $65^{\circ} \mathrm{C}$, and tert-butyl nitrite $(16 \mathrm{~mL})$ was added into the mixture dropwisely by a dropping funnel. After a 5-hour reaction at $65^{\circ} \mathrm{C}$, the mixture was cooled down to room temperature. The acetonitrile was removed by a rotary evaporator, and the residue was extracted with dilute aqueous sulfuric acid solution and dichloromethane. The organic layer was collected and dried with anhydrous magnesium sulfate. The organic solvent was removed by rotary evaporator, and the residue was purified via column chromatography yielding 1,4-dibromoanthraquinone as yellow solid. A mixture of 1,4-dibromoanthraquinone $(2.01 \mathrm{~g}, 5.46 \mathrm{mmol})$, sodium borohydride $(0.82 \mathrm{~g}, 21.6 \mathrm{mmol})$ and isopropanol $(50 \mathrm{~mL})$ in a $100 \mathrm{~mL}$ round bottom flask was stirred with a stir bar for a short while at room temperature. Then the mixture was kept in an ice-water bath for 3 hours, and put at room temperature for another 1 hour. An extra $100 \mathrm{~mL}$ of water was added into the reaction and a white solid was formed and collected. Afterwards a mixture of the obtained solid, tin(II) chloride (2.8 g, $14.8 \mathrm{mmol})$ and acetic acid $(40 \mathrm{~mL})$ was refluxed for 4 hours. Then the mixture was cooled down to room temperature, and a $100 \mathrm{~mL}$ of water was added into the mixture to afford a solid. This solid was collected and futher purifed by column chromatography (Isohexane) yielding DHA-1 as a yellow solid $(0.65 \mathrm{~g}, 1.93 \mathrm{mmol})$ in a $35 \%$ yield.

$\mathbf{R}_{\mathbf{f}}\left(\mathbf{S i O}_{2}\right)$ : 0.60 (isohexane).

${ }^{1} \mathbf{H}$ NMR $\left(400 \mathrm{MHz}, \mathrm{CDCl}_{3}\right) \delta 8.84(\mathrm{~s}, 2 \mathrm{H}), 8.11(\mathrm{dd}, J=6.4,3.3 \mathrm{~Hz}, 2 \mathrm{H}), 7.63(\mathrm{~s}, 2 \mathrm{H}), 7.58(\mathrm{dd}, J=$ $6.5,3.2 \mathrm{~Hz}, 2 \mathrm{H})$.

${ }^{13} \mathrm{C}$ NMR $\left(101 \mathrm{MHz}, \mathrm{CDCl}_{3}\right) \delta 132.6,130.4,129,0,128.4,127.3,126.9,122.8$. 


\section{1,5-dibromoanthracene (DHA-2) ${ }^{4}$.}<smiles>Brc1cccc2cc3ccccc3cc12</smiles>

Chemical Formula: $\mathrm{C}_{14} \mathrm{H}_{8} \mathrm{Br} 2$ Molecular Weight: 336,0260

A mixture of 1,5-diaminoanthraquinone (5 g, $21 \mathrm{mmol})$, copper(II) bromide (10.1 g, $47.3 \mathrm{mmol})$ and acetonitrile $(100 \mathrm{~mL})$ in a $250 \mathrm{~mL}$ two-necked round bottom flask was stirred with a stir for a short while at room temperature. Tert-butylnitrite $(6.3 \mathrm{~mL}, 90 \%)$ was added under vigorous stirring and the mixture was heated to $65^{\circ} \mathrm{C}$ for $2.5 \mathrm{~h}$.. After the reaction, the mixture was cooled down to room temperature. The acetonitrile was removed by a rotary evaporator, and the residue was extracted with dilute aqueous sulfuric acid solution and dichloromethane. The organic layer was collected and dried with anhydrous magnesium sulfate. The organic solvent was removed by rotary evaporator, and the residue was purified via column chromatography yielding 1,5dibromoanthraquinone as yellow solid. A mixture of 1,4-dibromoanthraquinone (4 g, $5.46 \mathrm{mmol}$ ), sodium borohydride $(1.04 \mathrm{~g}, 27.4 \mathrm{mmol})$ and isopropanol $(75 \mathrm{~mL})$ in a $250 \mathrm{~mL}$ round bottom flask was stirred with a stir bar for a short while at room temperature. Then the mixture was kept in an icewater bath for 3 hours, and put at room temperature for another 1 hour. An extra $100 \mathrm{~mL}$ of water was added into the reaction and a white solid was formed and collected. Afterwards a mixture of the obtained solid, tin(II) chloride $(9.5 \mathrm{~g}, 50 \mathrm{mmol})$ and acetic acid $(100 \mathrm{~mL})$ was refluxed for 4 hours. Then the mixture was cooled down to room temperature, and a $100 \mathrm{~mL}$ of water was added into the mixture to afford a solid. This solid was collected and futher purifed by column chromatography (Isohexane) yielding BB-4 as a yellow solid (1.5 g, $4.82 \mathrm{mmol}$ ) in a $23 \%$ yield.

$\mathbf{R}_{\mathbf{f}}\left(\mathbf{S i O}_{2}\right): 0.70$ (isohexane).

${ }^{1} \mathbf{H}$ NMR $\left(400 \mathrm{MHz}_{\mathrm{CDCl}}\right) \delta 8.79(\mathrm{~s}, 2 \mathrm{H}), 8.05(\mathrm{~d}, J=8.6 \mathrm{~Hz}, 2 \mathrm{H}), 7.83(\mathrm{~d}, J=7.1 \mathrm{~Hz}, 2 \mathrm{H}), 7.35$ $(\mathrm{dd}, J=8.4,7.3 \mathrm{~Hz}, 2 \mathrm{H})$.

${ }^{13}$ C NMR (101 MHz, $\left.\mathrm{CDCl}_{3}\right) \delta 133.0,130.4,130.0,128.8,127.1,126.0,122.5$. 


\section{4,9-dibromo-1,2,3,6,7,8-hexahydropyrene ${ }^{5}$.}<smiles>Brc1cc2c3c(c(Br)cc4c3c1CCC4)CCC2</smiles>

Chemical Formula: $\mathrm{C}_{16} \mathrm{H}_{14} \mathrm{Br}_{2}$ Molecular Weight: 366,0960
$0.5 \mathrm{~g}$ of $1,2,3,6,7,8$-hexahydropyrene $(2.4 \mathrm{mmol})$ was added to $5 \mathrm{ml}$ of $\mathrm{CH}_{2} \mathrm{Cl}_{2}$ and stirred. $0,25 \mathrm{ml}$ of $\mathrm{Br}_{2}$ was added to $1 \mathrm{ml}$ of $\mathrm{CH}_{2} \mathrm{Cl}_{2}$, and this solution was slowly added by dropping. After 30 minutes of reaction, the solid was filtered and dried in vacuo. 4,9-dibromo1,2,3,6,7,8-hexahydropyrene was obtained as a white solid. (600 mg, $35 \%)$. 1H-NMR (300 MHz, CDCl3, $): 7.40(\mathrm{~s}, 2 \mathrm{H}), 3.06(\mathrm{t}, 4 \mathrm{H})$,

2.97(t, 4H), 1.99(m, 4H); EI+-Mass: 366.

${ }^{1}$ H NMR (400 MHz, C6 $\left.\mathrm{D}_{6}\right) \delta 7.25(\mathrm{~s}, 2 \mathrm{H}), 2.88(\mathrm{t}, J=6.3 \mathrm{~Hz}, 4 \mathrm{H}), 2.50-2.43(\mathrm{~m}, 4 \mathrm{H}), 1.62-1.50$ (m, 4H).

${ }^{13}$ C NMR (101 MHz, $\left.\mathrm{CDCl}_{3}\right) \delta 135.5,133.0,130.6,128.4,120.7,31.3,30.9,22.6$.

\section{4,9-dibromopyrene (DHA-3) $)^{5}$.}

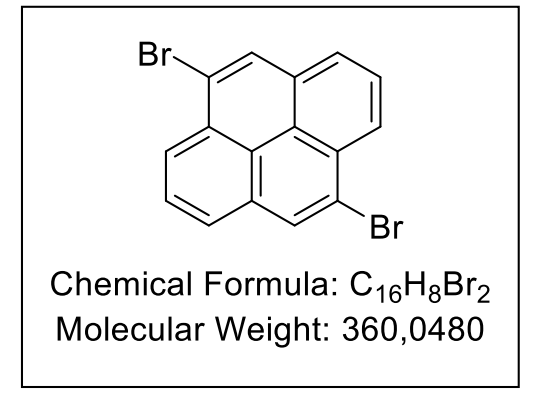

solid (250 mg, 44\%).

$\mathbf{R}_{\mathbf{f}}\left(\mathbf{S i O}_{2}\right)$ : 0.76 (Isohexane).

${ }^{1} \mathbf{H}$ NMR $\left(400 \mathrm{MHz}, \mathrm{CDCl}_{3}\right) \delta 8.65(\mathrm{~d}, J=7.9 \mathrm{~Hz}, 2 \mathrm{H}), 8.48(\mathrm{~s}, 2 \mathrm{H}), 8.21(\mathrm{~d}, J=7.5 \mathrm{~Hz}, 2 \mathrm{H}), 8.13$ (t, $J=7.8 \mathrm{~Hz}, 2 \mathrm{H}$ ).

${ }^{13}$ C NMR $\left(101 \mathrm{MHz} \mathrm{CDCl}_{3}\right) \delta 131.4,130.9,129.8,127.1,125.8,125.8,124.5,122.5$.
$0.5 \mathrm{~g}$ of 4,9-dibromo-1,2,3,6,7,8-hexahydropyrene $(1.37 \mathrm{mmol})$ and $0.93 \mathrm{mg}$ of DDQ were added to $10 \mathrm{ml}$ of anhydrous toluene under nitrogen atmosphere and stirred for reflux. After $4 \mathrm{~h}$ of reaction, temperature was reduced to room temperature. Toluene was evaporated under reduced pressure and the residue was purified by column chromatography (isohexane) yielding DHA-3 as a white 


\section{1,5-dibromonaphthalene (DHA-5) ${ }^{6}$.}

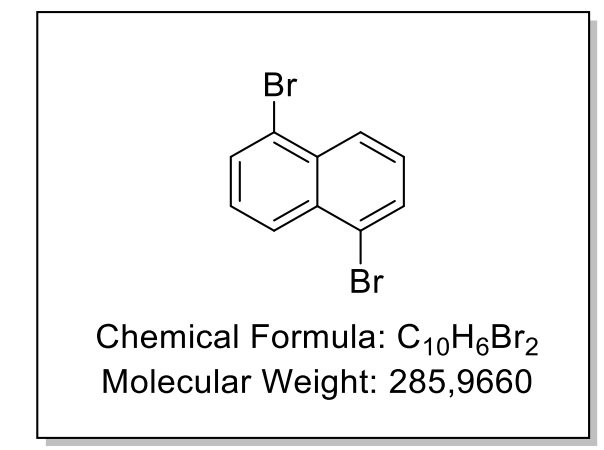

1,5-Diaminonaphthalene (4.00 g, $25 \mathrm{mmol})$ was combined with cooling with $100 \mathrm{~mL}$ of water and $6.6 \mathrm{~mL}$ of concentrated $\mathrm{H}_{2} \mathrm{SO}_{4}$. $\mathrm{NaNO}_{2}(3.9 \mathrm{~g}, 56 \mathrm{mmol})$ in water $(35 \mathrm{~mL})$ was charged in small portions, and the resulting mixture was stirred for $45 \mathrm{~min}$ at $0{ }^{\circ} \mathrm{C}$. This mixture was filtered, and the filtrate was added to $\mathrm{CuBr}$ (10.0 g, $70 \mathrm{mmol}$ ) which had been dissolved in a mixture of $48 \%$ $\mathrm{HBr}(150 \mathrm{~mL})$ and water $(150 \mathrm{~mL})$. After stirring at 0 "C $(1 \mathrm{~h})$, room temperature $(1-2 \mathrm{~h})$, and then $70{ }^{\circ} \mathrm{C}$ (30 min), nitrogen evolution had ceased. The reaction was extracted with DCM, and concentrated in vacuo and the product was purified by silica gel column chromatography, eluting with isohexane yielding BB-7a as white solid in 15\% (1 g, $3.50 \mathrm{mmol})$.

${ }^{1} \mathbf{H}$ NMR $\left(300 \mathrm{MHz}, \mathrm{CDCl}_{3}\right) \delta 8.26(\mathrm{~d}, J=8.7 \mathrm{~Hz}, 1 \mathrm{H}), 7.85(\mathrm{dd}, J=7.3,0.7 \mathrm{~Hz}, 1 \mathrm{H}), 7.43(\mathrm{dd}, J=$ 8.3, $7.5 \mathrm{~Hz}, 1 \mathrm{H})$.

${ }^{13} \mathrm{C}$ NMR $\left(76 \mathrm{MHz}, \mathrm{CDCl}_{3}\right) \delta 133.1,130.9,127.4,127.3,123.0$.

\section{2,5-dibromoterephthalaldehyde (BS-1) ${ }^{7}$}<smiles>O=Cc1cc(Br)c(C=O)cc1Br</smiles>

Chemical Formula: $\mathrm{C}_{8} \mathrm{H}_{4} \mathrm{Br}_{2} \mathrm{O}_{2}$ Molecular Weight: 291.9260
Sulfuric acid $(28 \mathrm{ml})$ was added dropwise to a suspension containing 1,4-Dibromo-2,5-dimethylbenzene (8.0 g), acetic acid $(40 \mathrm{ml})$ and acetic anhydride $(80 \mathrm{ml})$ at $0{ }^{\circ} \mathrm{C} . \mathrm{CrO}_{3}(12 \mathrm{~g})$ was then added to the mixture in portions. The resulting mixture was stirred vigorously at this temperature for a further $5 \mathrm{~h}$ until the reaction was completed. The greenish slurry was poured into ice water and

filtered. The white solid was washed with water. The diacetate was then hydrolyzed by refluxing with a mixture of water $(40 \mathrm{ml})$, ethanol $(40 \mathrm{ml})$ and sulfuric acid $(4 \mathrm{ml})$ for $5 \mathrm{~h}$. After the mixture had cooled, the pale yellow product was separated by filtration. The crude product was purified by recrystallization from chloroform $(4.3 \mathrm{~g}$, yield $48.6 \%)$

${ }^{1} \mathbf{H}$ NMR $\left(400 \mathrm{MHz}, 283 \mathrm{~K}, \mathrm{CDCl}_{3}\right) \delta 10.34(\mathrm{~s} ; 2 \mathrm{H}), 8.16(\mathrm{~s} ; 2 \mathrm{H})$.

${ }^{13} \mathrm{C}$ NMR $\left(101 \mathrm{MHz}, \mathrm{CDCl}_{3}\right) \delta 189.8,137.3,135.0,125.5$. 


\section{Synthesis of precursors P1-P9 for DPEX reaction.}

2,2'-(anthracene-1,4-diyl)dibenzaldehyde (P1).

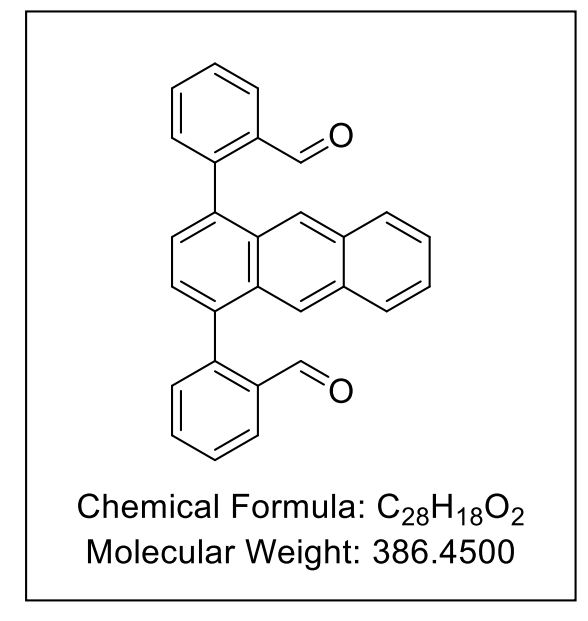

A $100 \mathrm{~mL}$ round bottom flask equipped with a magnetic stir bar and a condenser was charged with 1,4-dibromoanthracene (DHA1) $(336 \mathrm{mg}, 1 \mathrm{mmol})$, 2-formylphenylboronic acid (FBA-1) (375 mg, $2.5 \mathrm{mmol}), \mathrm{K}_{2} \mathrm{CO}_{3}(1.11 \mathrm{~g}, 7.67 \mathrm{mmol})$ and $\mathrm{Pd}\left(\mathrm{PPh}_{3}\right)_{4}$ $(85.0 \mathrm{mg}, 74 \mu \mathrm{mol})$. The solids were suspended in $3: 1 / 0.3$ toluene $/ \mathrm{MeOH} / \mathrm{H}_{2} \mathrm{O}(18.5 \mathrm{~mL})$, degassed and the atmosphere was exchanged by argon. The mixture was brought to reflux for $16 \mathrm{~h}$. The mixture was diluted with DCM $(50 \mathrm{~mL})$ and washed with $\mathrm{H}_{2} \mathrm{O}(2 \times 50 \mathrm{~mL})$. The aqueous layer was extracted with DCM (1x

$80 \mathrm{~mL}$ ) and the combined organics were dried over $\mathrm{MgSO}_{4}$. The solvent was evaporated and the product was purified by silica gel column chromatography, eluting with isohexane- isohexane:EtOAc (4:1) yielding $\mathbf{P 1}$ as pale yellow solid in $87 \%$ (338 $\mathrm{mg}, 0.87 \mathrm{mmol}$ )

$\mathbf{R}_{\mathbf{f}}\left(\mathbf{S i O}_{2}\right): 0.28$ (4:1 isohexanes/EtOAc).

${ }^{1} \mathbf{H}$ NMR $\left(400 \mathrm{MHz}, \mathrm{CDCl}_{3}\right) \delta 9.77(\mathrm{~d}, J=0.7 \mathrm{~Hz}, 1 \mathrm{H}), 9.76(\mathrm{~d}, J=0.7 \mathrm{~Hz}, 1 \mathrm{H}), 8.21(\mathrm{dt}, J=7.8,1.6$ $\mathrm{Hz}, 2 \mathrm{H}), 8.12$ (s, 2H), $7.86-7.77$ (m, 4H), 7.69 (t, $J=7.9 \mathrm{~Hz}, 2 \mathrm{H}), 7.61$ (dd, $J=9.4,4.2 \mathrm{~Hz}, 2 \mathrm{H}$ ), $7.48(\mathrm{~d}, J=2.1 \mathrm{~Hz}, 2 \mathrm{H}), 7.46-7.41(\mathrm{~m}, 2 \mathrm{H})$.

${ }^{13}$ C NMR (101 MHz, Acetone) $\delta$ 192.1, 191.9, 144.70, 144.68, 137.46, 137.42, 136.24, 136.17, 134.94, 134.91, 133.0, 132.87, 132.82, 132.45, 132.40, 129.6, 129.2, 128.14, 128.13, 128.0, 127.3, $126.51,126.49$.

HRMS (APPI; Toluene): Chemical Formula: $\mathrm{C}_{28} \mathrm{H}_{18} \mathrm{O}_{2}$ calc. 386.1301, found 386.1326. 


\section{2,2'-(anthracene-1,5-diyl)dibenzaldehyde (P2).}

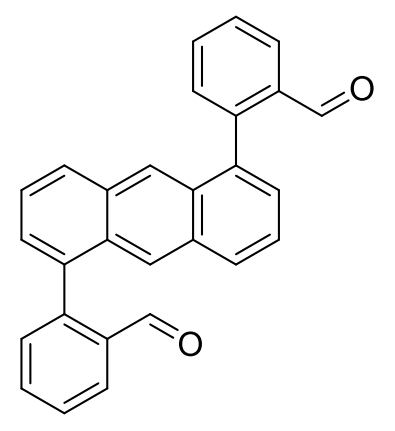

Chemical Formula: $\mathrm{C}_{28} \mathrm{H}_{18} \mathrm{O}_{2}$ Molecular Weight: 386.4500
A $100 \mathrm{~mL}$ round bottom flask equipped with a magnetic stir bar and a condenser was charged with 1,5-dibromoanthracene (DHA2) (336 mg, $1 \mathrm{mmol}$ ), 2-formylphenylboronic acid (FBA-1) (375 mg, $2.5 \mathrm{mmol}), \mathrm{K}_{2} \mathrm{CO}_{3}(1.11 \mathrm{~g}, 7.67 \mathrm{mmol})$ and $\mathrm{Pd}\left(\mathrm{PPh}_{3}\right)_{4}$ (85.0 $\mathrm{mg}, 74 \mu \mathrm{mol})$. The solids were suspended in 3:1/0.3 toluene/ $\mathrm{MeOH} / \mathrm{H}_{2} \mathrm{O}(18.5 \mathrm{~mL})$, degassed and the atmosphere was exchanged by argon. The mixture was brought to reflux for $16 \mathrm{~h}$. The mixture was diluted with DCM $(50 \mathrm{~mL})$ and washed with $\mathrm{H}_{2} \mathrm{O}(2 \times 50 \mathrm{~mL})$. The aqueous layer was extracted with DCM (1x

$80 \mathrm{~mL}$ ) and the combined organics were dried over $\mathrm{MgSO}_{4}$. The solvent was evaporated and the product was purified by silica gel column chromatography, eluting with isohexane- isohexane:EtOAc (4:1) yielding $\mathbf{P 2}$ as pale yellow solid in $18 \%$ (70 $\mathrm{mg}, 0.18 \mathrm{mmol})$

$\mathbf{R}_{\mathbf{f}}\left(\mathbf{S i O}_{2}\right)$ : 0.20 (4:1 isohexanes/EtOAc).

${ }^{1} \mathbf{H}$ NMR $\left(400 \mathrm{MHz}, \mathrm{CD}_{2} \mathrm{Cl}_{2}\right) \delta 9.69(\mathrm{dd}, J=2.4,0.7 \mathrm{~Hz}, 2 \mathrm{H}), 8.22-8.17(\mathrm{~m}, 4 \mathrm{H}), 7.96(\mathrm{~d}, J=8.6$ $\mathrm{Hz}, 2 \mathrm{H}), 7.84(\mathrm{tt}, J=7.5,1.5 \mathrm{~Hz}, 2 \mathrm{H}), 7.72(\mathrm{t}, J=7.6 \mathrm{~Hz}, 2 \mathrm{H}), 7.60$ (ddd, $J=7.5,2.0,0.9 \mathrm{~Hz}, 2 \mathrm{H})$, $7.56(\mathrm{dd}, J=8.5,6.7 \mathrm{~Hz}, 2 \mathrm{H}), 7.47(\mathrm{dd}, J=6.7,1.0 \mathrm{~Hz}, 2 \mathrm{H})$.

${ }^{13} \mathrm{C}$ NMR $\left(101 \mathrm{MHz}, \mathrm{CD}_{2} \mathrm{Cl}_{2}\right) \delta 192.38,192.36,144.6,136.28,136.26,135.78,135.75,134.5,134.4$, $132.55,132.52,132.1,129.73,129.71,129.1,128.90,128.86,127.91,127.87,126.2,125.5$.

HRMS (APPI; Toluene): Chemical Formula: $\mathrm{C}_{28} \mathrm{H}_{18} \mathrm{O}_{2}$ calc. 386.1301, found 386.1305. 


\section{3,3'-(anthracene-1,5-diyl)bis(2-naphthaldehyde) (P3).}<smiles>O=Cc1cc2ccccc2cc1-c1cccc2cc3c(-c4cc5ccccc5cc4C=O)cccc3cc12</smiles>

Chemical Formula: $\mathrm{C}_{36} \mathrm{H}_{22} \mathrm{O}_{2}$ Molecular Weight: 486,5700
A $25 \mathrm{~mL}$ round bottom flask equipped with a magnetic stir bar and a condenser was charged with 1,5dibromonaphtalene (DHA-2) (64 mg, 0,19 $\mu \mathrm{mol})$, 2formylnaphthyl-3-boronic acid pinacol ester (FBA-2) $(80$ $\mathrm{mg}, 286 \mu \mathrm{mol}), \mathrm{K}_{2} \mathrm{CO}_{3}(116 \mathrm{mg}, 790 \mu \mathrm{mol})$ and $\mathrm{Pd}\left(\mathrm{PPh}_{3}\right)_{4}$ ( $7 \mathrm{mg}, 8 \mu \mathrm{mol})$. The solids were suspended in $2: 1: 1$ toluene $/ \mathrm{MeOH} / \mathrm{H}_{2} \mathrm{O} \quad(3.5 \mathrm{~mL})$, degassed and the atmosphere was exchanged by argon. The mixture was brought to reflux for $16 \mathrm{~h}$. The mixture was diluted with DCM $(30 \mathrm{~mL})$ and washed with $\mathrm{H}_{2} \mathrm{O}(50 \mathrm{~mL})$. The aqueous layer was extracted with DCM (1x $50 \mathrm{~mL})$ and the combined organics were dried over $\mathrm{MgSO}_{4}$. The solvent was evaporated and the product was purified by silica gel column chromatography, eluting with isohexane-isohexane:EtOAc (5:1) yielding P3 as pale yellow solid in $40 \%$ (26 mg, $53.0 \mathrm{mmol})$

$\mathbf{R}_{\mathbf{f}}\left(\mathbf{S i O}_{2}\right)$ : 0.36 (4:1 isohexanes/EtOAc).

${ }^{1} \mathbf{H}$ NMR $\left(300 \mathrm{MHz}, \mathrm{CDCl}_{3}\right) \delta 9.79(\mathrm{~s}, 0.8 \mathrm{H}), 9.76(\mathrm{~s}, 1 \mathrm{H}), 8.75(\mathrm{~d}, J=4.1 \mathrm{~Hz}, 2 \mathrm{H}), 8.18(\mathrm{~d}, J=8.1$ $\mathrm{Hz}, 2 \mathrm{H}), 8.15(\mathrm{~d}, J=3.6 \mathrm{~Hz}, 2 \mathrm{H}), 8.04-7.94(\mathrm{~m}, 4 \mathrm{H}), 7.91-7.85(\mathrm{~m}, 2 \mathrm{H}), 7.77-7.65$ (m, 4H), 7.51 $(\mathrm{dd}, J=4.0,1.1 \mathrm{~Hz}, 4 \mathrm{H})$.

${ }^{13} \mathrm{C}$ NMR $\left(76 \mathrm{MHz}, \mathrm{CDCl}_{3}\right) \delta 192.05,192.03,138.72,138.68,135.95,135.93,135.88,132.99$, $132.95,132.32$, 132.31, 131.9, 131.79, 131.76, 130.9, 130.8, 130.1, 130.0, 129.6, 129.4, 129.34, $129.28,129.21,129.18,128.3,128.2,128.02,127.95,127.27,127.25,125.6,125.0$.

HRMS (APPI; Toluene): Chemical Formula: $\mathrm{C}_{36} \mathrm{H}_{22} \mathrm{O}_{2}$, calc. 486.1612, found 486.1614 . 


\section{2,2'-(phenanthrene-1,8-diyl)dibenzaldehyde (P4).}

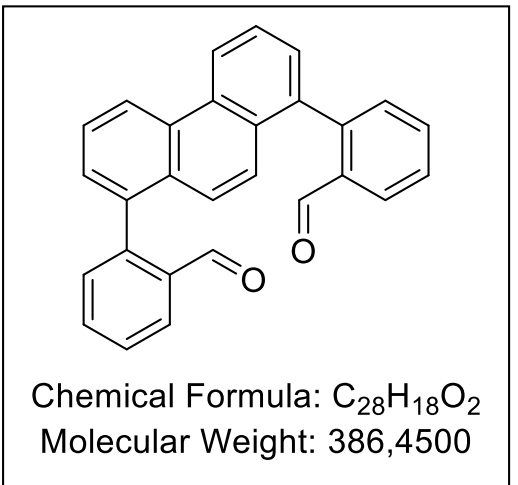

A $100 \mathrm{~mL}$ round bottom flask equipped with a magnetic stir bar and a condenser was charged with 1,8-dibromophenanthrene (DHA-3) (150 mg, $\quad 0.45 \mathrm{mmol}), \quad 2$-formylphenylboronic acid (FBA-1) (165 mg, $1.13 \mathrm{mmol}), \mathrm{K}_{2} \mathrm{CO}_{3}(500 \mathrm{mg}, 3.45 \mathrm{mmol})$ and $\mathrm{Pd}\left(\mathrm{PPh}_{3}\right)_{4}$ (37.0 $\mathrm{mg}, 33 \mu \mathrm{mol})$. The solids were suspended in $3: 1 / 0.3$ toluene $/ \mathrm{MeOH} / \mathrm{H}_{2} \mathrm{O}(8.8 \mathrm{~mL})$, degassed and the atmosphere was exchanged by argon. The mixture was brought to reflux for $16 \mathrm{~h}$. The mixture was diluted with DCM $(50 \mathrm{~mL})$ and washed with $\mathrm{H}_{2} \mathrm{O}(2 \times 50 \mathrm{~mL})$. The aqueous layer was extracted with DCM $(1 \times 80 \mathrm{~mL})$ and the combined organics were dried over $\mathrm{MgSO}_{4}$. The solvent was evaporated and the product was purified by silica gel column chromatography, eluting with isohexaneisohexane:EtOAc (4:1) yielding $\mathbf{P 4}$ as pale yellow solid in $86 \%$ yield (150 $\mathrm{mg}, 0.39 \mathrm{mmol})$.

$\mathbf{R f}_{\mathbf{f}}\left(\mathbf{S i O}_{2}\right): 0.22$ (4:1 isohexanes/EtOAc).

${ }^{1}$ H NMR (400 MHz, Acetone) $\delta 9.65(\mathrm{~d}, J=0.7 \mathrm{~Hz}, 1 \mathrm{H}), 9.64(\mathrm{~d}, J=0.7 \mathrm{~Hz}, 1 \mathrm{H}), 9.10(\mathrm{dd}, J=8.4$, $0.5 \mathrm{~Hz}, 2 \mathrm{H}), 8.04(\mathrm{dd}, J=7.9,1.1 \mathrm{~Hz}, 2 \mathrm{H}), 7.88(\mathrm{ddd}, J=8.3,7.2,0.9 \mathrm{~Hz}, 2 \mathrm{H}), 7.80$ (tt, $J=7.5,1.6$ $\mathrm{Hz}, 2 \mathrm{H}), 7.71-7.64(\mathrm{~m}, 4 \mathrm{H}), 7.53-7.48(\mathrm{~m}, 2 \mathrm{H}), 7.37(\mathrm{~d}, J=6.0 \mathrm{~Hz}, 2 \mathrm{H})$.

${ }^{13}$ C NMR (101 MHz, Acetone) $\delta$ 191.7, 137.50, 137.48, 135.89, 135.88, 134.8, 134.7, 132.83, 132.81, $131.53,131.49,131.44,130.3,130.2,129.44,129.43,128.10,128.06,127.50,127.46,125.7,124.6$.

HRMS (APPI; Toluene): Chemical Formula: $\mathrm{C}_{28} \mathrm{H}_{18} \mathrm{O}_{2}$ calc. 386.1301, found 386.1309.

\section{2,2'-(pyrene-4,9-diyl)dibenzaldehyde (P5).}

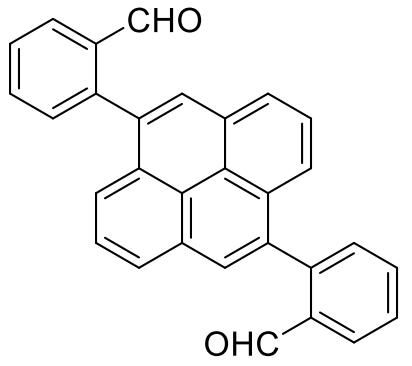

Chemical Formula: $\mathrm{C}_{30} \mathrm{H}_{18} \mathrm{O}_{2}$ Molecular Weight: 410,4720
A $100 \mathrm{~mL}$ round bottom flask equipped with a magnetic stir bar and a condenser was charged with 4,9-dibromopyrene (DHA-4) (60 mg, $0.17 \mathrm{mmol}$ ), 2-formylphenylboronic acid (FBA-1) (60 mg, $0.41 \mathrm{mmol}), \mathrm{K}_{2} \mathrm{CO}_{3}(120 \mathrm{mg}, 0.82 \mathrm{mmol})$ and $\mathrm{Pd}\left(\mathrm{PPh}_{3}\right)_{4}$ ( $5 \mathrm{mg}, 4.5 \mu \mathrm{mol})$. The solids were suspended in $2: 1 / 0.2$ toluene $/ \mathrm{MeOH} / \mathrm{H}_{2} \mathrm{O}(3.2 \mathrm{~mL})$, degassed and the atmosphere was exchanged by argon. The mixture was brought to reflux for $16 \mathrm{~h}$.

The mixture was diluted with DCM $(25 \mathrm{~mL})$ and washed with $\mathrm{H}_{2} \mathrm{O}(2 \times 30 \mathrm{~mL})$. The aqueous layer was extracted with DCM (1x $50 \mathrm{~mL})$ and the combined organics were dried over $\mathrm{MgSO}_{4}$. The solvent 
was evaporated and the product was purified by silica gel column chromatography, eluting with isohexane- isohexane:EtOAc (4:1) P5 as pale yellow solid in 35\% yield (25 mg, $0.39 \mathrm{mmol}$ )

$\mathbf{R}_{\mathbf{f}}\left(\mathbf{S i O}_{2}\right): 0.18$ (4:1 isohexanes/EtOAc).

${ }^{1} \mathbf{H}$ NMR $\left(400 \mathrm{MHz}, \mathrm{CDCl}_{3}\right) \delta 9.76(\mathrm{~d}, J=0.7 \mathrm{~Hz}, 1 \mathrm{H}), 9.74(\mathrm{~d}, J=0.7 \mathrm{~Hz}, 1 \mathrm{H}), 8.17-8.10(\mathrm{~m}, 4 \mathrm{H})$, $8.01(\mathrm{~s}, 2 \mathrm{H}), 7.90(\mathrm{t}, J=7.7 \mathrm{~Hz}, 2 \mathrm{H}), 7.78(\mathrm{~d}, J=7.9 \mathrm{~Hz}, 2 \mathrm{H}), 7.71(\mathrm{tdd}, J=7.5,3.4,1.4 \mathrm{~Hz}, 2 \mathrm{H})$, $7.62-7.53(\mathrm{~m}, 4 \mathrm{H})$.

${ }^{13} \mathrm{C}$ NMR $\left(101 \mathrm{MHz}, \mathrm{CDCl}_{3}\right) \delta 191.92,191.88,143.85,143.83,135.24,135.18,135.1,134.0,133.9$, $131.81,131.79,131.4,130.4,129.6,128.6,127.5,126.7,126.1,124.5,124.35,124.34$.

HRMS (APPI; Toluene): Chemical Formula: $\mathrm{C}_{30} \mathrm{H}_{18} \mathrm{O}_{2}$ calc. 410.1301, found 410.1305.

\section{3,3'-(pyrene-4,9-diyl)bis(2-naphthaldehyde) (P6).}

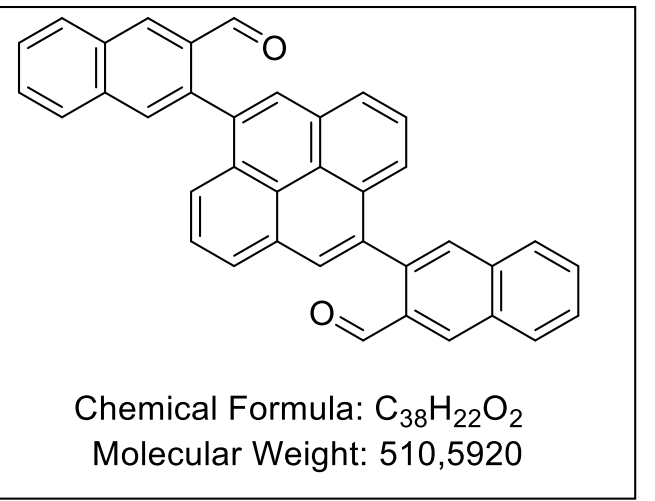

atmosphere was exchanged by argon. The mixture was brought to reflux for $16 \mathrm{~h}$. The mixture was diluted with DCM (25 mL) and washed with $\mathrm{H}_{2} \mathrm{O}(2 \times 30 \mathrm{~mL})$. The aqueous layer was extracted with DCM (1x $50 \mathrm{~mL})$ and the combined organics were dried over $\mathrm{MgSO}_{4}$. The solvent was evaporated and the product was purified by silica gel column chromatography, eluting with isohexaneisohexane:EtOAc (4:1) yielding $\mathbf{P 6}$ as pale yellow solid in 12\% yield (5 mg, 9,8 $\mu \mathrm{mol}$ )

$\mathbf{R}_{\mathbf{f}}\left(\mathbf{S i O}_{2}\right)$ : 0.22 (4:1 isohexanes/EtOAc).

${ }^{1}$ H NMR $\left(500 \mathrm{MHz}, \mathrm{DMSO}, 120{ }^{\circ} \mathrm{C}\right) \delta 9.94(\mathrm{~d}, J=8.1 \mathrm{~Hz}, 2 \mathrm{H}), 8.74(\mathrm{~s}, 4 \mathrm{H}), 8.37(\mathrm{~d}, J=7.4 \mathrm{~Hz}$, 2H), $8.32-8.27(\mathrm{~m}, 4 \mathrm{H}), 8.21(\mathrm{~s}, 2 \mathrm{H}), 8.12(\mathrm{~d}, J=8.9 \mathrm{~Hz}, 2 \mathrm{H}), 8.02(\mathrm{t}, J=7.7 \mathrm{~Hz}, 2 \mathrm{H}), 7.79$ (ddd, $J$ $=8.1,6.8,1.4 \mathrm{~Hz}, 2 \mathrm{H}), 7.75(\mathrm{ddd}, J=8.1,6.9,1.3 \mathrm{~Hz}, 2 \mathrm{H})$.

HRMS (APPI; Toluene): Chemical Formula: $\mathrm{C}_{38} \mathrm{H}_{22} \mathrm{O}_{2}$ calc. 510.1614, found 510.1609. 


\section{[2,1':5',2''-ternaphthalene]-3,3"-dicarbaldehyde (P7).}

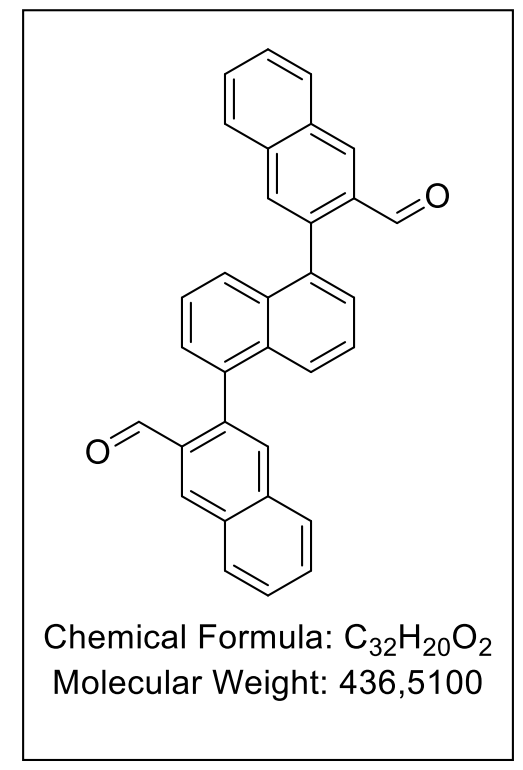

A $25 \mathrm{~mL}$ round bottom flask equipped with a magnetic stir bar and a condenser was charged with 1,5-diiodonaphtalene (DHA-5a) (27 mg, $71 \mu \mathrm{mol}), 2$-formylnaphthyl-3-boronic acid pinacol ester (FBA-2) (45 mg, $161 \mu \mathrm{mol}), \mathrm{K}_{2} \mathrm{CO}_{3}(66 \mathrm{mg}, 446 \mu \mathrm{mol})$ and $\mathrm{Pd}\left(\mathrm{PPh}_{3}\right)_{4}(4.5 \mathrm{mg}, 3.9 \mu \mathrm{mol})$. The solids were suspended in 2:1 toluene/MeOH $(1.5 \mathrm{~mL})$, degassed and the atmosphere was exchanged by argon. The mixture was brought to reflux for $16 \mathrm{~h}$. The mixture was diluted with DCM $(20 \mathrm{~mL})$ and washed with $\mathrm{H}_{2} \mathrm{O}$ $(50 \mathrm{~mL})$. The aqueous layer was extracted with DCM $(1 \times 50 \mathrm{~mL})$ and the combined organics were dried over $\mathrm{MgSO}_{4}$. The solvent was evaporated and the product was purified by silica gel column chromatography, eluting with isohexane-isohexane:EtOAc (4:1) yielding P7 as pale yellow solid in $42 \%$ (13 mg, $29.8 \mathrm{mmol})$

$\mathbf{R}_{\mathbf{f}}\left(\mathbf{S i O}_{2}\right)$ : 0.32 (4:1 isohexanes/EtOAc).

${ }^{1} \mathbf{H}$ NMR $\left(400 \mathrm{MHz} \mathrm{CDCl}_{3}, 293 \mathrm{~K}\right): \delta 9.74(\mathrm{~s}, 1 \mathrm{H}), 9.71(\mathrm{~s}, 1 \mathrm{H}), 8.62(\mathrm{~d}, J=6.5 \mathrm{~Hz}, 2 \mathrm{H}), 8.06(\mathrm{~d}, J$ $=8.1 \mathrm{~Hz}, 2 \mathrm{H}), 7.88(\mathrm{dd}, J=18.8,11.8 \mathrm{~Hz}, 4 \mathrm{H}), 7.63(\mathrm{ddd}, J=9.6,5.8,2.4 \mathrm{~Hz}, 2 \mathrm{H}), 7.56(\mathrm{t}, J=12.6$ $\mathrm{Hz}, 4 \mathrm{H}), 7.44$ (ddd, $J=15.1,10.4,5.5 \mathrm{~Hz}, 4 \mathrm{H})$.

${ }^{13} \mathrm{C}$ NMR (101 MHz, $\left.\mathrm{CDCl}_{3}, 293 \mathrm{~K}\right): \delta$ 192.1, 191.9, 138.71, 138.69, 136.5, 135.8, 135.77, 133.0, $132.8,132.23,132.21,130.9,130.8,130.00,129.95,129.6,129.50,129.4,129.3,128.5,128.4,128.0$, $127.9,127.3,126.50,126.46,126.01,125.95$.

HRMS (APPI; Toluene): Chemical Formula: $\mathrm{C}_{32} \mathrm{H}_{20} \mathrm{O}_{2}$, calc. 436.1458, found 436.1460.

\section{2-(5-iodonaphthalen-1-yl)benzaldehyde.}

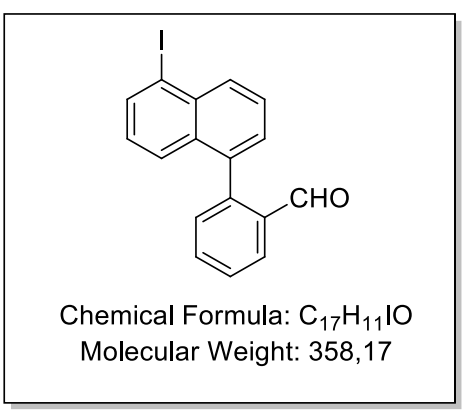

A $100 \mathrm{~mL}$ round bottom flask equipped with a magnetic stir bar and a condenser was charged with 1,5-diiodonaphtalene (DHA-5a) (190 mg, $0.5 \mathrm{mmol}$ ), 2-formylphenylboronic acid (FBA-1) (75 mg, $0.5 \mathrm{mmol}$ ), $\mathrm{K}_{2} \mathrm{CO}_{3}(345 \mathrm{mg}, 2.45 \mathrm{mmol})$ and $\mathrm{Pd}\left(\mathrm{PPh}_{3}\right)_{4}(11.5 \mathrm{mg}, 10 \mu \mathrm{mol})$. The solids were suspended in 2:1/0.3 toluene/MeOH/ $\mathrm{H}_{2} \mathrm{O}(5 \mathrm{~mL})$, degassed and the atmosphere was exchanged by argon. The mixture was brought to reflux for $16 \mathrm{~h}$. The mixture was diluted with DCM $(50 \mathrm{~mL})$ and washed with $\mathrm{H}_{2} \mathrm{O}(50 \mathrm{~mL})$. The 
aqueous layer was extracted with DCM (2x $50 \mathrm{~mL})$ and the combined organics were dried over $\mathrm{MgSO}_{4}$. The solvent was evaporated and the product was purified by silica gel column chromatography, eluting with isohexane-isohexane:EtOAc (4:1) yielding 2-(5-bromonaphthalen-1yl)benzaldehyde as oil in 56\% (100 $\mathrm{mg}, 0.28 \mathrm{mmol})$.

${ }^{1} \mathbf{H}$ NMR $\left(400 \mathrm{MHz}, \mathrm{CDCl}_{3}\right) \delta 9.60(\mathrm{~d}, J=0.7 \mathrm{~Hz}, 1 \mathrm{H}), 8.25(\mathrm{dt}, J=8.7,1.0 \mathrm{~Hz}, 1 \mathrm{H}), 8.14-8.11(\mathrm{~m}$; 2H), $7.72(\mathrm{td}, J=7.5,1.5 \mathrm{~Hz}, 1 \mathrm{H}), 7.69-7.59(\mathrm{~m}, 2 \mathrm{H}), 7.51-7.46(\mathrm{~m}, 2 \mathrm{H}), 7.44(\mathrm{dd}, J=7.6,0.9$ $\mathrm{Hz}, 1 \mathrm{H}), 7.11(\mathrm{dd}, J=8.5,7.3 \mathrm{~Hz}, 1 \mathrm{H})$.

${ }^{13} \mathrm{C}$ NMR $\left(101 \mathrm{MHz}, \mathrm{CDCl}_{3}\right) \delta$ 191.6, 143.5, 137.9, 136.1, 134.8, 134.2, 133.7, 133.4, 133.0, 131.7, $129.0,128.4,127.6,127.3,126.9,126.8,100.2$.

HRMS (APPI; Toluene): Chemical Formula: $\mathrm{C}_{17} \mathrm{H}_{11} \mathrm{IO}$, calc. 358.1736, found 358.1739.

\section{5-(2-formylphenyl)-[1,2'-binaphthalene]-3'-carbaldehyde (P8).}

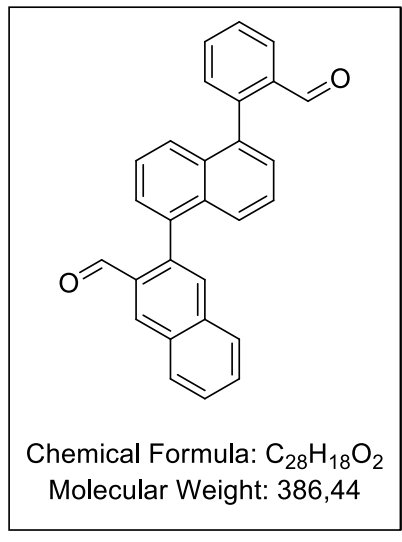

A $25 \mathrm{~mL}$ round bottom flask equipped with a magnetic stir bar and a condenser was charged with 2-(5-iodonaphthalen-1-yl)benzaldehyde. (96 mg, $27 \mu \mathrm{mol}), 2$-formylnaphthyl-3-boronic acid pinacol ester (FBA-2) (90 mg, $322 \mu \mathrm{mol}), \mathrm{K}_{2} \mathrm{CO}_{3}(120 \mathrm{mg}, 802 \mu \mathrm{mol})$ and $\mathrm{Pd}\left(\mathrm{PPh}_{3}\right)_{4}(8 \mathrm{mg}$, $6.9 \mu \mathrm{mol})$. The solids were suspended in $2: 1$ toluene/MeOH $(5 \mathrm{~mL})$, degassed and the atmosphere was exchanged by argon. The mixture was brought to reflux for $16 \mathrm{~h}$. The mixture was diluted with DCM $(20 \mathrm{~mL})$ and washed with $\mathrm{H}_{2} \mathrm{O}(50 \mathrm{~mL})$. The aqueous layer was extracted with DCM (1x

$50 \mathrm{~mL}$ ) and the combined organics were dried over $\mathrm{MgSO}_{4}$. The solvent was evaporated and the product was purified by silica gel column chromatography, eluting with isohexane-isohexane:EtOAc (4:1) yielding P8 as pale yellow solid in 86\% (90 mg, $23.3 \mathrm{mmol})$

$\mathbf{R}_{\mathbf{f}}\left(\mathbf{S i O}_{2}\right)$ : 0.32 (4:1 isohexanes/EtOAc).

${ }^{1} \mathbf{H}$ NMR $\left(300 \mathrm{MHz}, \mathrm{CDCl}_{3}\right) \delta 9.71(\mathrm{~d}, J=3.5 \mathrm{~Hz}, 1 \mathrm{H}), 9.63(\mathrm{dd}, J=13.2,0.9 \mathrm{~Hz}, 1 \mathrm{H}), 8.61(\mathrm{~d}, J=$ $4.0 \mathrm{~Hz}, 1 \mathrm{H}), 8.12-8.03(\mathrm{~m}, 2 \mathrm{H}), 7.93-7.82(\mathrm{~m}, 2 \mathrm{H}), 7.74-7.34(\mathrm{~m}, 11 \mathrm{H})$.

${ }^{13} \mathrm{C}$ NMR $\left(76 \mathrm{MHz}, \mathrm{CDCl}_{3}\right) \delta 192.02,191.99,191.8,144.2,144.1,138.61,138.59,136.5,135.98$, $135.97,135.8,135.7,134.87,134.85,133.8,133.7,133.0,132.7,132.68,132.67,132.21,132.20$, $131.8,131.7,130.83,130.79,130.0,129.9,129.6,129.5,129.4,129.3,128.6,128.5,128.4,128.0$, $127.9,127.3,127.2,126.58,126.56,126.43,126.40,126.01,125.98,125.9,125.8$. 
HRMS (APPI; Toluene): Chemical Formula: $\mathrm{C}_{28} \mathrm{H}_{18} \mathrm{O}_{2}$, calc.386.1301, found 386.1311.

\section{2-(5-bromonaphthalen-1-yl)benzaldehyde.}<smiles>O=Cc1ccccc1-c1cccc2c(Br)cccc12</smiles>

Chemical Formula: $\mathrm{C}_{17} \mathrm{H}_{11} \mathrm{BrO}$ Molecular Weight: 311,1780
A $100 \mathrm{~mL}$ round bottom flask equipped with a magnetic stir bar and a condenser was charged with 1,5-dibromonaphtalene (DHA-5) (700 mg, $2.45 \mathrm{mmol}$ ), 2-formylphenylboronic acid (FBA-1) (375 mg, $2.5 \mathrm{mmol}), \mathrm{K}_{2} \mathrm{CO}_{3}(1.69 \mathrm{~g}, 11.67 \mathrm{mmol})$ and $\mathrm{Pd}\left(\mathrm{PPh}_{3}\right)_{4}(56 \mathrm{mg}, 49 \mu \mathrm{mol})$. The solids were suspended in $2: 1 / 0.3$ toluene/ $\mathrm{MeOH} / \mathrm{H}_{2} \mathrm{O} \quad(25 \mathrm{~mL})$, degassed and the atmosphere was exchanged by argon. The mixture was brought to reflux for $16 \mathrm{~h}$. The mixture was diluted with DCM $(50 \mathrm{~mL})$ and washed with $\mathrm{H}_{2} \mathrm{O}(50 \mathrm{~mL})$. The aqueous layer was extracted with DCM $(2 \times 50 \mathrm{~mL})$ and the combined organics were dried over $\mathrm{MgSO}_{4}$. The solvent was evaporated and the product was purified by silica gel column chromatography, eluting with isohexane-isohexane:EtOAc (4:1) yielding 2-(5-bromonaphthalen-1yl)benzaldehyde as white solid in $99 \%$ (750 $\mathrm{mg}, 2.41 \mathrm{mmol}$ ).

${ }^{1} \mathbf{H}$ NMR $\left(400 \mathrm{MHz}, \mathrm{CDCl}_{3}\right) \delta 9.61(\mathrm{~d}, J=0.7 \mathrm{~Hz}, 1 \mathrm{H}), 8.40(\mathrm{dt}, J=8.7,1.0 \mathrm{~Hz}, 1 \mathrm{H}), 8.13(\mathrm{dd}, J=$ 7.8, 1.2 Hz, 1H), $7.83(\mathrm{dd}, J=7.4,1.0 \mathrm{~Hz}, 1 \mathrm{H}), 7.70(\mathrm{ddd}, J=15.8,8.0,6.5 \mathrm{~Hz}, 2 \mathrm{H}), 7.65-7.59(\mathrm{~m}$, $1 \mathrm{H}), 7.52-7.42(\mathrm{~m}, 3 \mathrm{H}), 7.30-7.23(\mathrm{~m}, 1 \mathrm{H})$.

${ }^{13} \mathrm{C}$ NMR $\left(101 \mathrm{MHz}, \mathrm{CDCl}_{3}\right) \delta 191.7,143.7,136.0,134.8,134.0,133.7,132.0,131.7,130.4,129.0$, $128.5,127.9,127.4,126.9,126.4,125.9,123.5$.

HRMS (APPI; Toluene): Chemical Formula: $\mathrm{C}_{17} \mathrm{H}_{11} \mathrm{BrO}_{2}$, calc. 309.9988, found 309.9985. 
2-(5-(4,4,5,5-tetramethyl-1,3,2-dioxaborolan-2-yl)naphthalen-1-yl)benzaldehyde.

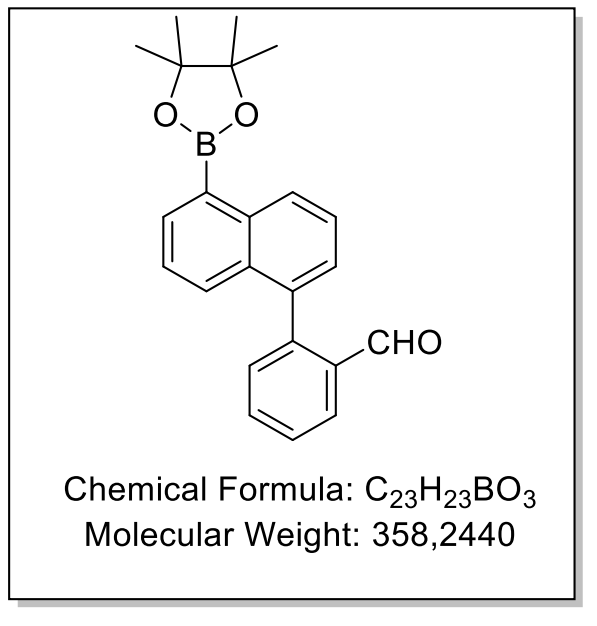

isohexane- isohexane:EtOAc (4:1), yielding 2-(5-(4,4,5,5-tetramethyl-1,3,2-dioxaborolan-2yl)naphthalen-1-yl)benzaldehyde as yellow oil which slowly solidifies to an yellow solid in $46 \%$ (400 mg, $1.11 \mathrm{mmol}$ )

${ }^{1} \mathbf{H}$ NMR $\left(400 \mathrm{MHz}, \mathrm{CDCl}_{3}\right) \delta 9.62(\mathrm{~d}, J=0.8 \mathrm{~Hz}, 1 \mathrm{H}), 8.90(\mathrm{t}, J=0.9 \mathrm{~Hz}, 1 \mathrm{H}), 8.12(\mathrm{dd}, J=7.1,1.2$ $\mathrm{Hz}, 2 \mathrm{H}), 7.70(\mathrm{td}, J=7.5,1.5 \mathrm{~Hz}, 1 \mathrm{H}), 7.66-7.56(\mathrm{~m}, 3 \mathrm{H}), 7.48-7.39(\mathrm{~m}, 3 \mathrm{H}), 1.46(\mathrm{~s}, 12 \mathrm{H})$.

${ }^{13} \mathrm{C}$ NMR $\left(101 \mathrm{MHz}, \mathrm{CDCl}_{3}\right) \delta 192.1,144.7,136.9,135.9,135.6,134.9,133.6,132.5,131.8,129.3$, $129.1,128.1,127.9,127.0,125.8,125.4,83.9,25.0,24.9$.

HRMS (APPI; Toluene): Chemical Formula: $\mathrm{C}_{17} \mathrm{H}_{11} \mathrm{BrO}_{2}$, calc. 357.9849, found 357.9844. 


\section{2,5-bis(5-(2-formylphenyl)naphthalen-1-yl)terephthalaldehyde (P9).}

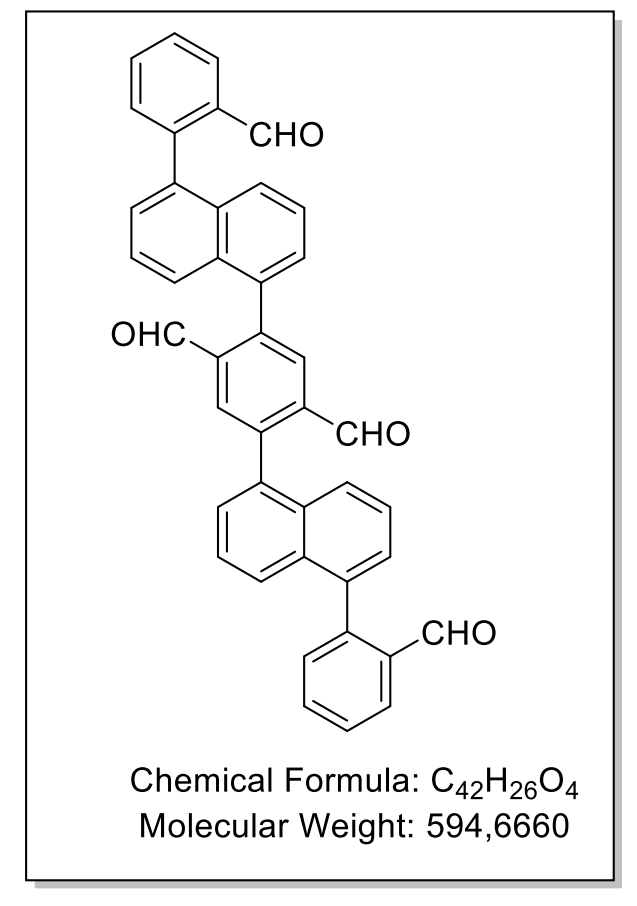

A $100 \mathrm{~mL}$ round bottom flask equipped with a magnetic stir bar and a condenser was charged with 2,5dibromoterephthalaldehyde (BS-1) $(68 \mathrm{mg}, 0.23 \mathrm{mmol}), 2-(5-$ (4,4,5,5-tetramethyl-1,3,2-dioxaborolan-2-yl)naphthalen-1yl)benzalde hyde $(300 \mathrm{mg}, 0.83 \mathrm{mmol}), \mathrm{K}_{2} \mathrm{CO}_{3}(236 \mathrm{mg}$, $1.62 \mathrm{mmol})$ and $\mathrm{Pd}\left(\mathrm{PPh}_{3}\right)_{4}(63 \mathrm{mg}, 55 \mu \mathrm{mol})$. The solids were suspended in 3:1:1 toluene/EtOH/ $\mathrm{H}_{2} \mathrm{O}(10 \mathrm{~mL})$, degassed and the atmosphere was exchanged by argon. The mixture was brought to reflux for $16 \mathrm{~h}$. The mixture was diluted with DCM (50 mL) and washed with $\mathrm{H}_{2} \mathrm{O}(50 \mathrm{~mL})$. The aqueous layer was extracted with DCM (2x $50 \mathrm{~mL})$ and the combined organics were dried over $\mathrm{MgSO}_{4}$. The solvent was evaporated and the product was purified by silica gel column chromatography, eluting with isohexane-isohexane:EtOAc (4:1) yielding P9 as white solid in 27\% (37 mg, $0.06 \mathrm{mmol}$ ). ${ }^{1} \mathbf{H}$ NMR $\left(300 \mathrm{MHz}, \mathrm{CDCl}_{3}\right) \delta 9.95-9.81(\mathrm{~m}, 2 \mathrm{H}), 9.74(\mathrm{dd}, J=4.6,2.4 \mathrm{~Hz}, 2 \mathrm{H}), 8.29(\mathrm{dd}, J=7.1$, $3.6 \mathrm{~Hz}, 2 \mathrm{H}), 8.18(\mathrm{~d}, J=7.5 \mathrm{~Hz}, 2 \mathrm{H}), 7.83-7.74(\mathrm{~m}, 2 \mathrm{H}), 7.62$ (ddd, $J=27.5,13.8,6.2 \mathrm{~Hz}, 18 \mathrm{H})$.

${ }^{13}$ C NMR (101 MHz, $\left.\mathrm{CDCl}_{3}\right) \delta$ 191.8, 191.2, 191.0, 143.83, 143.82, 143.46, 143.45, 143.43, 143.40, 137.93, 137.90, 136.37, 136.34, 134.9, 134.8, 134.61, 134.58, 133.84, 133.81, 132.91, 132.89, 131.8, $131.7,130.88,130.86,128.78,128.76,128.5,127.4,127.25,127.23,126.34,126.31,126.1,126.03$, 126.02 .

HRMS (APPI; Toluene): Chemical Formula: $\mathrm{C}_{42} \mathrm{H}_{28} \mathrm{O}_{4}$, calc. 594.1826, found 594.1823. 


\section{Synthesis of nanographenes.}

\section{Dibenzo[h,rst $]$ pentaphene (NG1).}

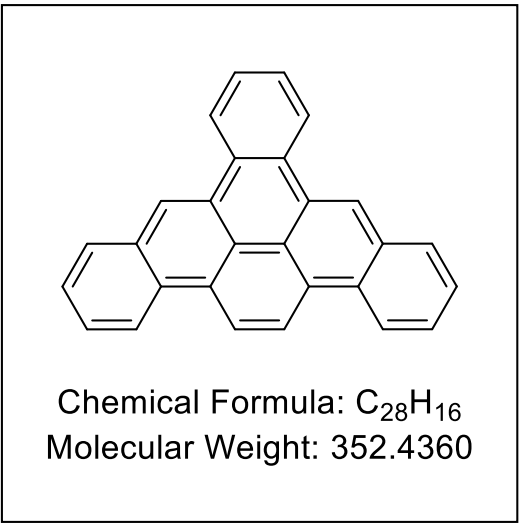

A $250 \mathrm{~mL}$ round bottom flask equipped with a magnetic stir bar was charged with the 2,2'-(anthracene-1,4-diyl)dibenzaldehyde (P1) (20.0 mg; $51.8 \mu \mathrm{mol})$, and dissolved in $\mathrm{CH}_{2} \mathrm{Cl}_{2}(100 \mathrm{~mL})$. While stirring, a solution of $\mathrm{SnCl}_{2} 2 \mathrm{H}_{2} \mathrm{O}(500 \mathrm{mg}, 2.22 \mathrm{mmol})$ in $i-\mathrm{PrOH}$ $(2.0 \mathrm{~mL})$ was added at $\mathrm{rt}$. Then, conc. $\mathrm{H}_{2} \mathrm{SO}_{4}(1.0 \mathrm{~mL})$ was added and the mixture was allowed to stir at $\mathrm{rt}$ with protection from daylight for $18 \mathrm{~h}$. The mixture was quenched with water $(100 \mathrm{~mL})$. Vigorous mixing was necessary at this point. The layers were separated, and the aqueous layer was extracted with $\mathrm{CH}_{2} \mathrm{Cl}_{2}(50 \mathrm{~mL})$. The organic layers were combined, and DCM was evaporated under reduced pressure. The residue was purified by silicagel column chromatography, eluting with isohexane-DCM 10:1, yielding NG1 as an off-white solid in 43 $\%$ (8 mg, $22.7 \mu \mathrm{mol})$.

$\mathbf{R}_{\mathbf{f}}\left(\mathbf{S i O}_{2}\right): 0.70$ (9:1 isohexanes/DCM).

${ }^{1} \mathbf{H}$ NMR $\left(400 \mathrm{MHz}, o-\mathrm{C}_{6} \mathrm{D}_{4} \mathrm{Cl}_{2}\right) \delta 9.10(\mathrm{~s}, 2 \mathrm{H}), 8.99(\mathrm{~s}, 2 \mathrm{H}), 8.92(\mathrm{~d}, J=7.7 \mathrm{~Hz}, 2 \mathrm{H}), 8.71(\mathrm{dd}, J=$ $5.2,3.5 \mathrm{~Hz}, 2 \mathrm{H}), 8.22(\mathrm{~d}, J=7.2 \mathrm{~Hz}, 2 \mathrm{H}), 7.82-7.70(\mathrm{~m}, 4 \mathrm{H}), 7.65(\mathrm{dd}, J=5.6,2.9 \mathrm{~Hz}, 2 \mathrm{H})$.

${ }^{13} \mathrm{C}$ NMR (101 MHz, $\left.\mathrm{C}_{2} \mathrm{D}_{2} \mathrm{Cl}_{4}\right) \delta 131.2,130.5,129.0,128.8,128.3,128.1,126.8,126.7,123.9,123.8$, $122.8,122.1,120.7,120.2$.

MS (LDI): $m / z$ (rel. int. $)=352.49[\mathrm{M}]^{+}(100)$.

HRMS (APPI; Toluene): Chemical Formula: $\mathrm{C}_{28} \mathrm{H}_{16}$ calc. 352.1247, found 352.1248.

UV/Vis (Toluene, $293 \mathrm{~K}): \lambda[\mathrm{nm}])=297,307,323,348,365,385$.

Fluo $\left(\mathrm{CH}_{2} \mathrm{Cl}_{2}, 293 \mathrm{~K}\right): \lambda$ [nm] (rel. int. [\%]) = 419 (100), 445 (90), 473 (26), 505 (4.5). 


\section{Dibenzo[fg,qr]pentacene (NG2).}

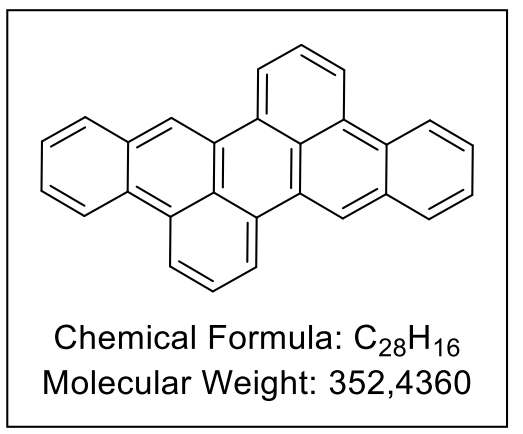

A $250 \mathrm{~mL}$ round bottom flask equipped with a magnetic stir bar was charged with the 2,2'-(anthracene-1,5-diyl)dibenzaldehyde (P2) (20.0 mg; $51.8 \mu \mathrm{mol}$ ), and dissolved in $\mathrm{CH}_{2} \mathrm{Cl}_{2}(100 \mathrm{~mL})$. While stirring, a solution of $\mathrm{SnCl}_{2} 2 \mathrm{H}_{2} \mathrm{O}(500 \mathrm{mg}, 2.22 \mathrm{mmol})$ in $i-\mathrm{PrOH}$ $(2.0 \mathrm{~mL})$ was added at rt. Then, conc. $\mathrm{H}_{2} \mathrm{SO}_{4}(1.0 \mathrm{~mL})$ was added and the mixture was allowed to stir at rt with protection from daylight for

$18 \mathrm{~h}$. The mixture was quenched with water $(100 \mathrm{~mL})$. Vigorous mixing was necessary at this point. The layers were separated, and the aqueous layer was extracted with $\mathrm{CH}_{2} \mathrm{Cl}_{2}(50 \mathrm{~mL})$. The organic layers were combined, and DCM was evaporated under reduced pressure. The residue was purified by silicagel column chromatography, eluting with isohexane-DCM 10:1, yielding NG2 as a yellowbrown solid in $53 \%(10 \mathrm{mg}, 28 \mu \mathrm{mol})$.

$\mathbf{R}_{\mathbf{f}}\left(\mathbf{S i O}_{2}\right): 0.70$ (9:1 isohexanes/DCM).

${ }^{1} \mathbf{H}$ NMR $\left(400 \mathrm{MHz}, \mathrm{CD}_{2} \mathrm{Cl}_{2}\right) \delta 8.61(\mathrm{~d}, J=8.1 \mathrm{~Hz}, 2 \mathrm{H}), 8.57(\mathrm{~d}, J=9.1 \mathrm{~Hz}, 2 \mathrm{H}), 8.53-8.47(\mathrm{~m}$, $4 \mathrm{H}), 7.94-7.89(\mathrm{~m}, 2 \mathrm{H}), 7.73(\mathrm{t}, J=8.0 \mathrm{~Hz}, 2 \mathrm{H}), 7.60-7.52$ (m, 4H).

${ }^{13}$ C NMR $\left(151 \mathrm{MHz}, \mathrm{CD}_{2} \mathrm{Cl}_{2}\right) \delta 132.7,131.7,131.53,130.45,129.47,129.41,127.7,127.6,127.5$, $127.4,123.20,123.18,122.1,121.8$.

MS (LDI): $m / z($ rel. int. $)=352.02[\mathrm{M}]^{+}(100)$.

HRMS (APPI; Toluene): Chemical Formula: $\mathrm{C}_{28} \mathrm{H}_{16}$ calc. 352.1247, found 352.1250.

UV/Vis (Toluene, $293 \mathrm{~K}): \lambda[\mathrm{nm}])=290,302,386,307,433$.

Fluo $\left(\mathrm{CH}_{2} \mathrm{Cl}_{2}, 293 \mathrm{~K}\right): \lambda[\mathrm{nm}]($ rel. int. [\%]) $=449$ (75), 470 (100), 499 (29). 


\section{Dibenzo[hi,wx]heptacene (NG3).}

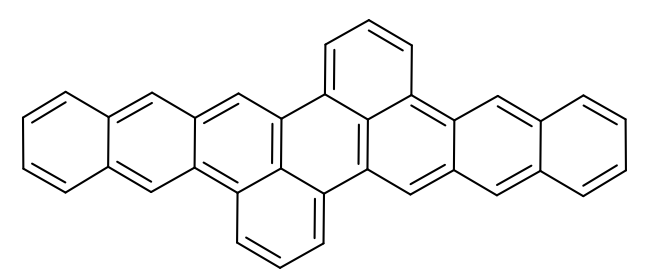

Chemical Formula: $\mathrm{C}_{36} \mathrm{H}_{20}$ Molecular Weight: 452,5560
A $250 \mathrm{~mL}$ round bottom flask equipped with a magnetic stir bar was charged with the precursor 3,3'(anthracene-1,5-diyl)bis(2-naphthaldehyde) (P3) (10.0 $\mathrm{mg} ; 20.5 \mu \mathrm{mol})$ and dissolved in $\mathrm{CH}_{2} \mathrm{Cl}_{2}(50 \mathrm{~mL})$. While stirring, a solution of $\mathrm{SnCl}_{2} \cdot 2 \mathrm{H}_{2} \mathrm{O}(250 \mathrm{mg}$, $1.11 \mathrm{mmol})$ in $i$-PrOH $(1.0 \mathrm{~mL})$ was added at $\mathrm{rt}$. Then,

conc. $\mathrm{H}_{2} \mathrm{SO}_{4}(0.5 \mathrm{~mL})$ was added and the mixture is allowed to stir at $\mathrm{rt}$ with protection from daylight for $18 \mathrm{~h}$. The mixture was quenched with water $(100 \mathrm{~mL})$. Vigorous mixing was necessary at this point. The layers were separated, and the aqueous layer was extracted with $\mathrm{CH}_{2} \mathrm{Cl}_{2}(50 \mathrm{~mL})$. The combined $\mathrm{CH}_{2} \mathrm{Cl}_{2}$ layers were diluted with $\mathrm{MeOH}(15 \mathrm{~mL})$ and the $\mathrm{CH}_{2} \mathrm{Cl}_{2}$ was removed on the rotavap at atmospheric pressure at $50{ }^{\circ} \mathrm{C}$. The formed precipitate in the $\mathrm{MeOH}$ layer was centrifuged, the $\mathrm{MeOH}$ layer was decanted and the solid was washed again with $\mathrm{MeOH}$. The NG3 was dried in vacuo and obtained as a yellow-green solid in $97 \%$ yield (9 mg $19.9 \mu \mathrm{mol})$.

${ }^{1} \mathrm{H}$ NMR $\left(400 \mathrm{MHz}, \mathrm{C}_{2} \mathrm{D}_{2} \mathrm{Cl}_{4}\right) \delta 9.10(\mathrm{~s}, 2 \mathrm{H}), 8.85(\mathrm{~d}, J=8.1 \mathrm{~Hz}, 2 \mathrm{H}), 8.62(\mathrm{~m}, 4 \mathrm{H}), 8.45(\mathrm{~s}, 2 \mathrm{H})$, $8.14-8.09(\mathrm{~m}, 2 \mathrm{H}), 8.07-8.02(\mathrm{~m}, 2 \mathrm{H}), 7.85(\mathrm{t}, J=7.9 \mathrm{~Hz}, 2 \mathrm{H}), 7.55(\mathrm{dd}, J=6.4,3.2 \mathrm{~Hz}, 4 \mathrm{H})$.

MS (LDI): $m / z$ (rel. int. $)=452$

HRMS (APPI; Toluene): Chemical Formula: $\mathrm{C}_{36} \mathrm{H}_{20}$ calc. 452.1560, found 452.1551.

UV/Vis (Toluene/MeOH, $293 \mathrm{~K}): \lambda[\mathrm{nm}])=290,318,343,354,369,395,417,444$.

Fluo $\left(\mathrm{CH}_{2} \mathrm{Cl}_{2}, 293 \mathrm{~K}\right): \lambda$ [nm] (rel. int. [\%]) = 488 (100), 514 (58), 564 (15). 


\section{Dibenzo[fg,ij]pentaphene (NG4).}

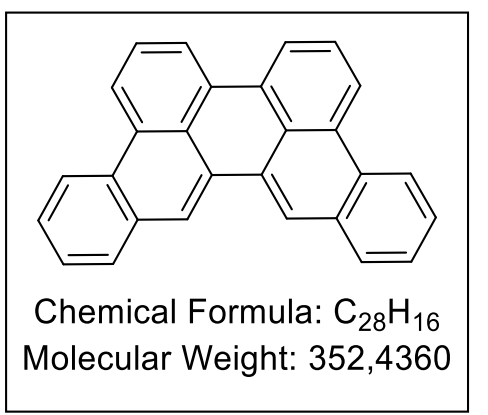

A $250 \mathrm{~mL}$ round bottom flask equipped with a magnetic stir bar was charged with the precursor 2,2'-(phenanthrene-1,8diyl)dibenzaldehyde (P4) $(20.0 \mathrm{mg} ; 51.8 \mu \mathrm{mol})$ and dissolved in $\mathrm{CH}_{2} \mathrm{Cl}_{2}(100 \mathrm{~mL})$. While stirring, a solution of $\mathrm{SnCl}_{2} \cdot 2 \mathrm{H}_{2} \mathrm{O}(500 \mathrm{mg}$, $2.22 \mathrm{mmol})$ in $i$-PrOH $(2.0 \mathrm{~mL})$ was added at $\mathrm{rt}$. Then, conc. $\mathrm{H}_{2} \mathrm{SO}_{4}$

$(1 \mathrm{~mL})$ was added and the mixture is allowed to stir at $\mathrm{rt}$ with protection from daylight for $18 \mathrm{~h}$. The mixture was quenched with water $(100 \mathrm{~mL})$. Vigorous mixing was necessary at this point. The layers were separated, and the aqueous layer was extracted with $\mathrm{CH}_{2} \mathrm{Cl}_{2}$ $(50 \mathrm{~mL})$. The organic layers were combined, and DCM was evaporated under reduced pressure yielding NG4 as a yellow-green solid in $98 \%$ yield $(18 \mathrm{mg}, 51 \mu \mathrm{mol})$.

${ }^{1}$ H NMR $\left(400 \mathrm{MHz}, \mathrm{o}-\mathrm{C}_{6} \mathrm{D}_{4} \mathrm{Cl}_{2}\right) \delta 8.59(\mathrm{~s}, 2 \mathrm{H}), 8.53-8.47(\mathrm{~m}, 6 \mathrm{H}), 8.28(\mathrm{~d}, J=7.5 \mathrm{~Hz}, 8 \mathrm{H}), 7.92$ $(\mathrm{dq}, J=6.9,3.5 \mathrm{~Hz}, 2 \mathrm{H}), 7.65(\mathrm{t}, J=7.9 \mathrm{~Hz}, 2 \mathrm{H}), 7.61-7.54(\mathrm{~m}, 4 \mathrm{H})$.

${ }^{13} \mathrm{C}$ NMR $\left(101 \mathrm{MHz}, \mathrm{C}_{2} \mathrm{D}_{2} \mathrm{Cl}_{4}\right) \delta 131.9,131.0,130.9,129.8,128.9,128.8,127.2,127.1,126.9,126.8$, $122.7,122.6,121.5,121.3$.

MS (LDI): $m / z($ rel. int. $)=352.48[\mathrm{M}]^{+}(100)$.

HRMS (APPI; Toluene): Chemical Formula: $\mathrm{C}_{28} \mathrm{H}_{16}$ calc. 352.1247, found 352.1251.

UV/Vis (Toluene, $293 \mathrm{~K}): \lambda[\mathrm{nm}])=290,302,391,412,430$.

Fluo $\left(\mathrm{CH}_{2} \mathrm{Cl}_{2}, 293 \mathrm{~K}\right): \lambda$ [nm] (rel. int. [\%]) = 458 (65), 477 (100), 510 (34). 


\section{Pyranthrene (NG5).}

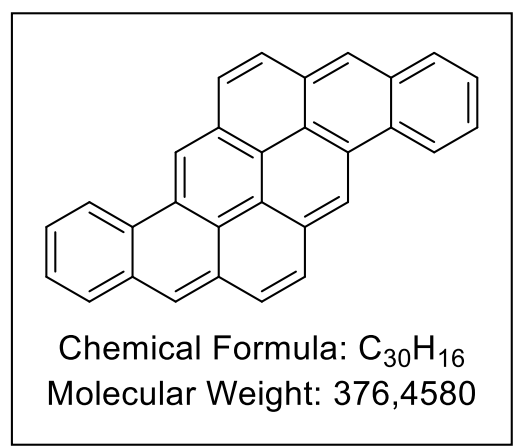

$250 \mathrm{~mL}$ round bottom flask equipped with a magnetic stir bar was charged with the precursor 2,2'-(pyrene-4,9-diyl)dibenzaldehyde (P5) $(10.0 \mathrm{mg} ; 24.4 \mu \mathrm{mol})$ and dissolved in $\mathrm{CH}_{2} \mathrm{Cl}_{2}(50 \mathrm{~mL})$. While stirring, a solution of $\mathrm{SnCl}_{2} 2 \mathrm{H}_{2} \mathrm{O}(250 \mathrm{mg}, 1.11 \mathrm{mmol})$ in $i$-PrOH $(1.0 \mathrm{~mL})$ was added at $\mathrm{rt}$. Then, conc. $\mathrm{H}_{2} \mathrm{SO}_{4}(0.5 \mathrm{~mL})$ was added and the mixture is allowed to stir at $\mathrm{rt}$ with protection from daylight for $18 \mathrm{~h}$. The mixture was quenched with water $(100 \mathrm{~mL})$. Vigorous mixing was necessary at this point. The layers were separated, and the aqueous layer was extracted with $\mathrm{CH}_{2} \mathrm{Cl}_{2}(50 \mathrm{~mL})$. The combined $\mathrm{CH}_{2} \mathrm{Cl}_{2}$ layers were diluted with $\mathrm{MeOH}(15 \mathrm{~mL})$ and the $\mathrm{CH}_{2} \mathrm{Cl}_{2}$ was removed on the rotavap at atmospheric pressure at $50{ }^{\circ} \mathrm{C}$. The formed precipitate in the $\mathrm{MeOH}$ layer was centrifuged, the $\mathrm{MeOH}$ layer was decanted and the solid was washed again with $\mathrm{MeOH}$. The NG5 was dried in vacuo and obtained as a red solid in $92 \%$ yield $(8.5 \mathrm{mg}, 22.4 \mu \mathrm{mol})$.

${ }^{1} \mathrm{H}$ NMR $\left(400 \mathrm{MHz}, \mathrm{CD}_{2} \mathrm{Cl}_{2}\right) \delta 9.64(\mathrm{~s}, 2 \mathrm{H}), 9.32(\mathrm{~d}, J=7.9 \mathrm{~Hz}, 2 \mathrm{H}), 8.56(\mathrm{~s}, 2 \mathrm{H}), 8.38(\mathrm{~d}, J=7.2$ $\mathrm{Hz}, 2 \mathrm{H}), 8.25$ (d, $J=9.2 \mathrm{~Hz}, 2 \mathrm{H}), 8.14(\mathrm{~d}, J=9.0 \mathrm{~Hz}, 2 \mathrm{H}), 8.00-7.93$ (m, 2H), 7.88 (dd, $J=12.5$, $5.6 \mathrm{~Hz}, 2 \mathrm{H})$.

MS (LDI): $\mathrm{m} / z($ rel. int. $)=376.05[\mathrm{M}]^{+}(100)$.

HRMS (APPI; Toluene): Chemical Formula: $\mathrm{C}_{30} \mathrm{H}_{16}$ calc. 376.1247, found 376.1236.

UV/Vis (Toluene, $293 \mathrm{~K}): \lambda[\mathrm{nm}])=288,303,320,335,354,383,407,431,461$.

Fluo $\left(\mathrm{CH}_{2} \mathrm{Cl}_{2}, 293 \mathrm{~K}\right): \lambda[\mathrm{nm}]($ rel. int. [\%]) $=476$ (71), $502(100), 558(21)$. 


\section{Dibenzo[b,o]pyranthrene (NG6).}

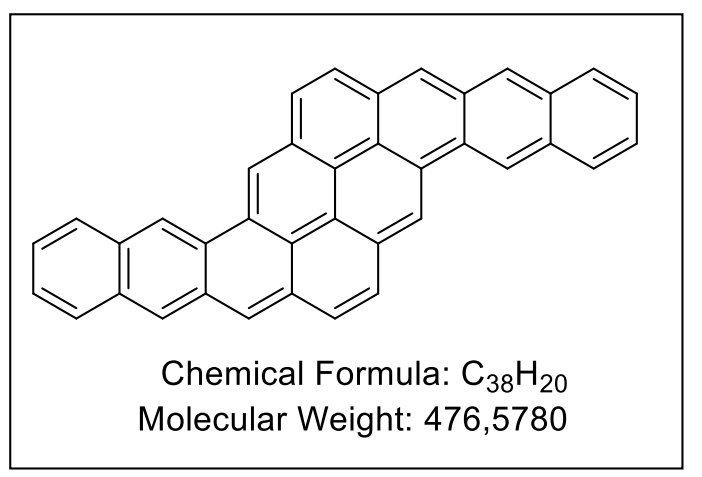

$100 \mathrm{~mL}$ round bottom flask equipped with a magnetic stir bar was charged with the precursor 3,3'-(pyrene-4,9diyl)bis(2-naphthaldehyde) (P6) $(5 \mathrm{mg} ; 0.98 \mu \mathrm{mol})$ and dissolved in $\mathrm{CH}_{2} \mathrm{Cl}_{2}(25 \mathrm{~mL})$. While stirring, a solution of $\mathrm{SnCl}_{2} 2 \mathrm{H}_{2} \mathrm{O}(125 \mathrm{mg}, 0.55 \mathrm{mmol})$ in $i-\mathrm{PrOH}(0.5 \mathrm{~mL})$ was added at $\mathrm{rt}$. Then, conc. $\mathrm{H}_{2} \mathrm{SO}_{4}(0.25 \mathrm{~mL})$ was added and the mixture is allowed to stir at $\mathrm{rt}$ with protection from daylight for $18 \mathrm{~h}$. The mixture was quenched with water $(50 \mathrm{~mL})$. Vigorous mixing was necessary at this point. The layers were separated, and the aqueous layer was extracted with $\mathrm{CH}_{2} \mathrm{Cl}_{2}(50 \mathrm{~mL})$. The combined $\mathrm{CH}_{2} \mathrm{Cl}_{2}$ layers were diluted with $\mathrm{MeOH}(15 \mathrm{~mL})$ and the $\mathrm{CH}_{2} \mathrm{Cl}_{2}$ was removed on the rotavap at atmospheric pressure at $50{ }^{\circ} \mathrm{C}$. The formed precipitate in the $\mathrm{MeOH}$ layer was centrifuged, the $\mathrm{MeOH}$ layer was decanted and the solid was washed again with $\mathrm{MeOH}$. The NG6 was dried in vacuo and obtained as a red solid in $90 \%$ yield $(4.2 \mathrm{mg}, 0.88 \mu \mathrm{mol})$.

${ }^{1} \mathbf{H}$ NMR $\left(500 \mathrm{MHz}, o-\mathrm{C}_{6} \mathrm{D}_{4} \mathrm{Cl}_{2}, 393 \mathrm{~K}\right) \delta 9.67(\mathrm{~s}, 2 \mathrm{H}), 9.53(\mathrm{~s}, 2 \mathrm{H}), 8.69(\mathrm{~s}, 2 \mathrm{H}), 8.38(\mathrm{~s}, 2 \mathrm{H}), 8.26$ (dd, $J=6.4,3.4 \mathrm{~Hz}, 2 \mathrm{H}), 8.15-8.08(\mathrm{~m}, 2 \mathrm{H}), 8.03(\mathrm{~d}, J=8.7 \mathrm{~Hz}, 2 \mathrm{H}), 7.91(\mathrm{~d}, J=9.1 \mathrm{~Hz}, 2 \mathrm{H}), 7.55$ $(\mathrm{dd}, J=6.7,3.0 \mathrm{~Hz}, 4 \mathrm{H})$.

MS (LDI): $m / z$ (rel. int. $)=476.10[\mathrm{M}]^{+}(100)$.

HRMS (MALDI): Chemical Formula: $\mathrm{C}_{38} \mathrm{H}_{20}$ calc. 476.1560, found 476.1572 .

UV/Vis (Toluene, $293 \mathrm{~K}): \lambda[\mathrm{nm}])=310,373,381,393,485,502,542$.

Fluo $(o-\mathrm{DCB}, 293 \mathrm{~K}): \lambda$ [nm] (rel. int. [\%]) = 557 (100), 598 (36), $653(8)$. 


\section{Tetraceno[2,1,12-qra]tetracene (NG7).}

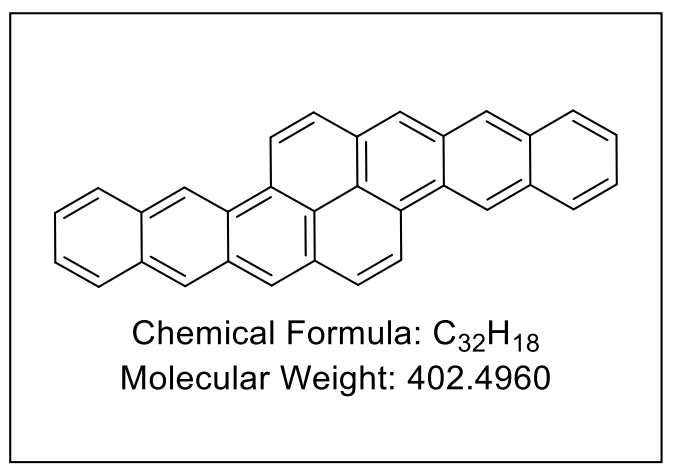

$250 \mathrm{~mL}$ round bottom flask equipped with a magnetic stir bar was charged with the precursor $\left[2,1^{\prime}: 5^{\prime}, 2^{\prime \prime}-\right.$ ternaphthalene]-3,3"-dicarbaldehyde. (13.0 mg; $29.8 \mu \mathrm{mol})$ and dissolved in $\mathrm{CH}_{2} \mathrm{Cl}_{2}(70 \mathrm{~mL})$. While stirring, a solution of $\mathrm{SnCl}_{2} 2 \mathrm{H}_{2} \mathrm{O}(325 \mathrm{mg}, 1.44 \mathrm{mmol})$ in $i-\mathrm{PrOH}(1.3 \mathrm{~mL})$ was added at rt. Then, conc. $\mathrm{H}_{2} \mathrm{SO}_{4}(0.65 \mathrm{~mL})$ was added and the mixture is allowed to stir at $\mathrm{rt}$ with protection from daylight for $18 \mathrm{~h}$. The mixture was quenched with water $(100 \mathrm{~mL})$. Vigorous mixing was necessary at this point. The layers were separated, and the aqueous layer was extracted with $\mathrm{CH}_{2} \mathrm{Cl}_{2}(50 \mathrm{~mL})$. The combined $\mathrm{CH}_{2} \mathrm{Cl}_{2}$ layers were diluted with $\mathrm{MeOH}(15 \mathrm{~mL})$ and the $\mathrm{CH}_{2} \mathrm{Cl}_{2}$ was removed on the rotavap at atmospheric pressure at $50{ }^{\circ} \mathrm{C}$. The formed precipitate in the $\mathrm{MeOH}$ layer was centrifuged, the $\mathrm{MeOH}$ layer was decanted and the solid was washed again with $\mathrm{MeOH}$. The NG7 was dried in vacuo and obtained as a dark blue solid in $92 \%$ yield $(11 \mathrm{mg}, 27.3 \mu \mathrm{mol})$.

${ }^{1} \mathrm{H}$ NMR $\left(500 \mathrm{MHz}, o-\mathrm{C}_{6} \mathrm{D}_{4} \mathrm{Cl}_{2}, 393 \mathrm{~K}\right) \delta 9.42(\mathrm{~s}, 1 \mathrm{H}), 8.94(\mathrm{~d}, J=9.4 \mathrm{~Hz}, 1 \mathrm{H}), 8.71(\mathrm{~s}, 1 \mathrm{H}), 8.63(\mathrm{~s}$, $1 \mathrm{H}), 8.22(\mathrm{~d}, J=9.5 \mathrm{~Hz}, 1 \mathrm{H}), 8.15-8.14(\mathrm{~m}, 1 \mathrm{H}), 8.03-8.02(\mathrm{~m}, 1 \mathrm{H}), 7.47-7.42(\mathrm{~m}, 2 \mathrm{H})$.

MS (LDI): $\mathrm{m} / z$ (rel. int. $)=402.06$

HRMS (APPI; Toluene): Chemical Formula: $\mathrm{C}_{32} \mathrm{H}_{18}$ calc. 402.1403, found 402.1388.

UV/Vis (Toluene, $293 \mathrm{~K}): \lambda[\mathrm{nm}])=316,330,346,384$, 495, 530, 573.

Fluo $\left(\mathrm{CH}_{2} \mathrm{Cl}_{2}, 293 \mathrm{~K}\right): \lambda$ [nm] (rel. int. [\%]) = $582(100), 631(59)$. 


\section{Anthra[2,1,9-qra]tetracene (NG8).}

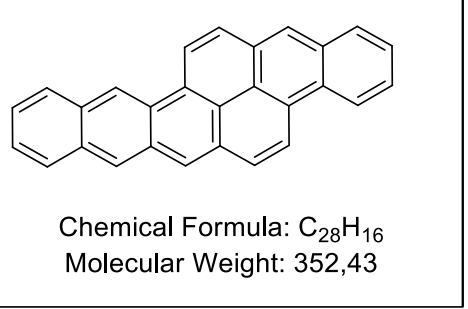

$250 \mathrm{~mL}$ round bottom flask equipped with a magnetic stir bar was charged with the precursor 5-(2-formylphenyl)-[1,2'binaphthalene]-3'-carbaldehyde (P8) $(20 \mathrm{mg} ; 51.8 \mu \mathrm{mol})$ and dissolved in $\mathrm{CH}_{2} \mathrm{Cl}_{2}(100 \mathrm{~mL})$. While stirring, a solution of $\mathrm{SnCl}_{2} \cdot 2 \mathrm{H}_{2} \mathrm{O}(500 \mathrm{mg}, 2.22 \mathrm{mmol})$ in $i-\mathrm{PrOH}(2.0 \mathrm{~mL})$ was added at rt. Then, conc. $\mathrm{H}_{2} \mathrm{SO}_{4}(1 \mathrm{~mL})$ was added and the mixture is allowed to stir at $\mathrm{rt}$ with protection from daylight for $18 \mathrm{~h}$. The mixture was quenched with water (100 mL). Vigorous mixing was necessary at this point. The layers were separated, and the aqueous layer was extracted with $\mathrm{CH}_{2} \mathrm{Cl}_{2}(50 \mathrm{~mL})$. The organic layers were combined, and DCM was evaporated under reduced pressure yielding NG8 as a red solid in 78\% yield (14 mg, $40 \mu \mathrm{mol})$.

${ }^{1} \mathbf{H}$ NMR $\left(300 \mathrm{MHz}, \mathrm{C}_{2} \mathrm{D}_{2} \mathrm{Cl}_{4}\right) \delta 9.64(\mathrm{~s}, 1 \mathrm{H}), 9.23(\mathrm{~d}, J=9.4 \mathrm{~Hz}, 1 \mathrm{H}), 8.99(\mathrm{~d}, J=8.4 \mathrm{~Hz}, 1 \mathrm{H}), 8.93$ (s, 1H), $8.87(\mathrm{~d}, J=9.5 \mathrm{~Hz}, 1 \mathrm{H}), 8.80(\mathrm{~s}, 1 \mathrm{H}), 8.73(\mathrm{~s}, 1 \mathrm{H}), 8.46(\mathrm{~d}, J=9.2 \mathrm{~Hz}, 1 \mathrm{H}), 8.39-8.20(\mathrm{~m}$, 4H), $7.91-7.75(\mathrm{~m}, 2 \mathrm{H}), 7.64(\mathrm{dd}, J=6.6,3.1 \mathrm{~Hz}, 2 \mathrm{H})$.

MS (LDI): $m / z$ (rel. int. $)=352.03$

HRMS (APPI; $\mathrm{CH}_{2} \mathrm{Cl}_{2}$ ): Chemical Formula: $\mathrm{C}_{28} \mathrm{H}_{16}$ calc. 352.1247, found 352.1241.

UV/Vis (Toluene, $293 \mathrm{~K}): \lambda[\mathrm{nm}])=311,323,349,367,426,452,482,516$.

Fluo $\left(\mathrm{CH}_{2} \mathrm{Cl}_{2}, 293 \mathrm{~K}\right): \lambda$ [nm] (rel. int. [\%]) = 530 (100), 561 (78), 605 (14). 


\section{Dianthra[2,1,9-jkl:2',1',9'-uva]pentacene (NG9).}

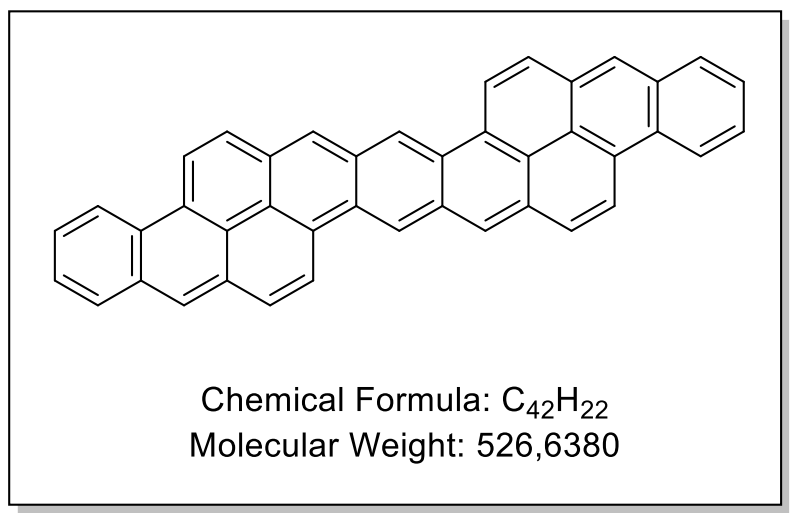

$250 \mathrm{~mL}$ round bottom flask equipped with a magnetic stir bar was charged with the precursor 2,5-bis(5-(2-formylphenyl)naphthalen-1-yl)tereph thalaldehyde (P8) (10.0 $\mathrm{mg} ; 16.8 \mu \mathrm{mol})$ and dissolved in $\mathrm{CH}_{2} \mathrm{Cl}_{2}(10 \mathrm{~mL})$. While stirring, a solution of $\mathrm{SnCl}_{2} 2 \mathrm{H}_{2} \mathrm{O}(500 \mathrm{mg}, 2.22 \mathrm{mmol})$ in $i$ $\mathrm{PrOH}(2.0 \mathrm{~mL})$ was added at rt. Then, conc. $\mathrm{H}_{2} \mathrm{SO}_{4}$ $(1.0 \mathrm{~mL})$ was added and the mixture is allowed to stir at $\mathrm{rt}$ with protection from daylight for $18 \mathrm{~h}$. The mixture was quenched with water $(100 \mathrm{~mL})$. Vigorous mixing was necessary at this point. The layers were separated, and the aqueous layer was extracted with $\mathrm{CH}_{2} \mathrm{Cl}_{2}(50 \mathrm{~mL})$. The combined $\mathrm{CH}_{2} \mathrm{Cl}_{2}$ layers were diluted with $\mathrm{MeOH}(15 \mathrm{~mL})$ and the $\mathrm{CH}_{2} \mathrm{Cl}_{2}$ was removed on the rotavap at atmospheric pressure at $50{ }^{\circ} \mathrm{C}$. The formed precipitate in the $\mathrm{MeOH}$ layer was centrifuged, the $\mathrm{MeOH}$ layer was decanted and the solid was washed again with $\mathrm{MeOH}$. The NG9 was dried, sublimated and obtained as a dark green solid in 79\% yield (7 mg, $13.2 \mu \mathrm{mol})$

MS (LDI): $m / z$ (rel. int. $)=526$

HRMS (MALDI): Chemical Formula: $\mathrm{C}_{42} \mathrm{H}_{24}$ calc. 526,1722, found 526.1720.

UV/Vis $(o-\mathrm{DCB}, 368 \mathrm{~K}): \lambda[\mathrm{nm}])=359,366,378,402,429,531,572,620$.

Fluo (o-DCB, $293 \mathrm{~K}): \lambda$ [nm] (rel. int. [\%]) = 631 (100), 682 (32), 730 (11). 


\section{Computations.}

All geometries were optimised with B3LYP functional and 6-311+G(d,p) basis set in Gaussian16 package ${ }^{1}$. Minimal character was proven by frequencies calculations at same level. All following singlepoint calculations were performed on these optimized geometries. Biradical character $y$ was calculated based on HONO and LUNO occupation numbers obtained by UHF/6-31+G(d,p) calculation with broken symmetry (guess=mix), applying formula of Nakano ${ }^{8}$. TD-calculations were performed with PBE, B3LYP and PBE0 functionals and cc-pVTZ basis set. Simulated UV-Vis spectra (TD-B3LYP and TD-PBE0, cc-pVTZ, 20 states that covers area till not less than $300 \mathrm{~nm}$ ) were combined with experimental and shown on the same figures below. Odd electron density ${ }^{9}(\mathrm{OED})$ was evaluated using the long-range corrected UBLYP with range-separating parameter $\mu$ of 0.33 bohr-1 method with $6-31+\mathrm{G}^{* 10}$ and $6-311+\mathrm{G}^{* * 11}$ basis sets (no significant difference from different basis sets was observed) with further treatment by Multiwfn software ${ }^{12}$. Fractional occupation number weighted electron density (FOD) analysis ${ }^{13,14}$ was performed in ORCA $^{15,16}$ with default settings (TPSS/def2-TZVP with a smearing temperature of $5000 \mathrm{~K}$ ).

\footnotetext{
${ }^{1}$ Gaussian 16, Revision B.01, M. J. Frisch, G. W. Trucks, H. B. Schlegel, G. E. Scuseria, M. A. Robb, J. R. Cheeseman, G. Scalmani, V. Barone, G. A. Petersson, H. Nakatsuji, X. Li, M. Caricato, A. V. Marenich, J. Bloino, B. G. Janesko, R. Gomperts, B. Mennuci, H. P. Hratchian, J. V. Ortiz, A. F. Izmaylov, J. L. Sonnenberg, D. Williams-Young, F. Ding, F. Lipparini, F. Egidi, J. Goings, B. Peng, A. Petrone, T. Henderson, D. Ranasinghe, V. G. Zakrzewski, J. Gao, N. Rega, G. Zheng, W. Liang, M. Hada, M. Ehara, K. Toyota, R. Fukuda, J. Hasegawa, M. Ishida, T. Nakajima, Y. Honda, O. Kitao, H. Nakai, T. Vreven, K. Throssell, J. A. Montgomery, Jr., J. E. Peralta, F. Ogliaro, M. J. Bearpark, J. J. Heyd, E. N. Brothers, K. N. Kudin, V. N. Staroverov, T. A. Keith, R. Kobayashi, J. Normand, K. Raghavachari, A. P. Rendell, J. C. Burant, S. S. Iyengar, J. Tomasi, M. Cossi, J. M. Millam, M. Klene, C. Adamo, R. Cammi, J. W. Ochterski, R. L. Martin, K. Morokuma, O. Farkas, J. B. Foresman, and D. J. Fox, Gaussian, Inc., Wallingford CT, 2016.
} 
Table S3. Optical, electrochemical and computational properties of NG1-9.

\begin{tabular}{|c|c|c|c|c|c|c|c|c|c|c|c|c|c|c|c|c|}
\hline & \multicolumn{2}{|c|}{ Occupation number } & $y$ & N_FOD & \multicolumn{2}{|c|}{$\begin{array}{c}\text { Optical gap } \\
\text { (experimental) }\end{array}$} & \multicolumn{2}{|c|}{ TD_PBE } & \multicolumn{2}{|c|}{ TD_PBE0 } & \multicolumn{2}{|c|}{ TD_B3LYP } & $\begin{array}{c}\begin{array}{c}\text { Electrochemical gap } \\
\text { (experimental) }\end{array} \\
\mathrm{eV}\end{array}$ & $\frac{\mathrm{PBE}}{\mathrm{eV}}$ & $\frac{\text { PBE0 }}{\mathrm{eV}}$ & $\frac{\text { B3LYP }}{\mathrm{eV}}$ \\
\hline NG1 & 1.55 & 0.45 & 0.15 & 0.365 & 3.14 & 395 & 2.80 & 443 & 3.24 & 383 & 3.15 & 394 & 2 & 2.33 & 3.70 & 3.42 \\
\hline NG4 & 1.51 & 0.49 & 0.19 & 0.4365 & 2.75 & 451 & 2.54 & 487 & 2.90 & 427 & 2.82 & 440 & 2.39 & 2.03 & 3.38 & 3.09 \\
\hline NG3 & 1.47 & 0.53 & 0.23 & 0.6809 & 2.68 & 461 & 2.19 & 566 & 2.81 & 441 & 2.71 & 458 & 2.11 & 1.92 & 3.22 & 2.94 \\
\hline NG5 & 1.42 & 0.58 & 0.28 & 0.583 & 2.64 & 470 & 2.36 & 525 & 2.68 & 462 & 2.61 & 475 & 3 & 1.74 & 2.93 & 2.68 \\
\hline NG6 & 1.36 & 0.64 & 0.37 & 0.9243 & 2.22 & 558 & 1.81 & 684 & 2.20 & 564 & 2.12 & 584 & 1.74 & 1.39 & 2.52 & 2.29 \\
\hline NG7 & 1.34 & 0.66 & 0.40 & 0.9201 & 2.10 & 590 & 1.63 & 763 & 1.99 & 624 & 1.92 & 646 & 1.72 & 1.24 & 2.41 & 2.17 \\
\hline NG9 & 1.31 & 0.69 & 0.43 & 1.1417 & 1.94 & 638 & 1.47 & 843 & 1.85 & 669 & 1.78 & 696 & 4 & 1.15 & 2.26 & 2.03 \\
\hline
\end{tabular}

${ }^{2}$ Electrochemical gap is bigger than electrochemical window of solvent.

${ }^{3}$ Electrochemical gap was not measured due to low solubility of NG5.

${ }^{4}$ Electrochemical gap was not measured due to low solubility of NG9. 


\section{References}

(1) Lungerich, D.; Papaianina, O.; Feofanov, M.; Liu, J.; Devarajulu, M.; Troyanov, S. I.; Maier, S.; Amsharov, K. Dehydrative $\pi$-Extension to Nanographenes with Zig-Zag Edges. Nat. Commun. 2018, $9(1), 4756$.

(2) Mallory, F. B.; Butler, K. E.; Evans, A. C.; Brondyke, E. J.; Mallory, C. W.; Yang, C.; Ellenstein, A. Phenacenes: A Family of Graphite Ribbons. 2. Syntheses of Some [7]Phenacenes and an [11]Phenacene by Stilbene-like Photocyclizations. J. Am. Chem. Soc. 1997, 119 (9), 2119-2124.

(3) Ho, J. H.; Chen, Y. H.; Chou, L. T.; Lai, P. W.; Chen, P. S. The Improvement of $\pi$-Conjugation by the Lateral Benzene of Anthracene and Naphthalene. Tetrahedron Lett. 2014, 55 (42), 5727-5731.

(4) Kang, S.; Lee, H.; Kim, B.; Kang, H.; Park, J. Synthesis and Property of New Blue Emitting Materials with Bulky Side Group. Mol. Cryst. Liq. Cryst. 2015, 618 (1), 66-73.

(5) Lee, J.; Park, J. Synthesis and Electroluminescence of Novel Pyrene-Fused Chromophores. Org. Lett. 2015, 17 (16), 3960-3963.

(6) Chambers, R. R.; Collins, C. J.; Maxwell, B. E. Reductive Debenzylation of 1-Benzylnaphthalene by a Na-K Alloy. J. Org. Chem. 1985, 50 (24), 4960-4963.

(7) Xie, Z.; Yang, B.; Liu, L.; Li, M.; Lin, D.; Ma, Y.; Cheng, G.; Liu, S. Experimental and Theoretical Studies of 2,5-Diphenyl-1,4-Distyrylbenzenes with All-Cis- And All-Trans Double Bonds: Chemical Structure Determination and Optical Properties. J. Phys. Org. Chem. 2005, 18 (9), 962-973.

(8) Kamada, K.; Ohta, K.; Shimizu, A.; Kubo, T.; Kishi, R.; Takahashi, H.; Botek, E.; Champagne, B.; Nakano, M. Singlet Diradical Character from Experiment. J. Phys. Chem. Lett. 2010, 1 (6), 937-940.

(9) Nakano, M.; Fukui, H.; Minami, T.; Yoneda, K.; Shigeta, Y.; Kishi, R.; Champagne, B.; Botek, E.; Kubo, T.; Ohta, K.; et al. (Hyper)Polarizability Density Analysis for Open-Shell Molecular Systems Based on Natural Orbitals and Occupation Numbers. Theor. Chem. Acc. 2011, 130 (4-6), 711-724.

(10) Fukuda, K.; Matsushita, N.; Minamida, Y.; Matsui, H.; Nagami, T.; Takamuku, S.; Kitagawa, Y.; Nakano, M. Impact of Diradical/Ionic Character on Third-Order Nonlinear Optical Property in Asymmetric Phenalenyl Dimers. ChemistrySelect 2017, 2 (6), 2084-2087.

(11) Rudebusch, G. E.; Zafra, J. L.; Jorner, K.; Fukuda, K.; Marshall, J. L.; Arrechea-Marcos, I.; Espejo, G. L.; Ponce Ortiz, R.; Gómez-García, C. J.; Zakharov, L. N.; et al. Diindeno-Fusion of an Anthracene as a Design Strategy for Stable Organic Biradicals. Nat. Chem. 2016, 8 (8), 753-759.

(12) Lu, T.; Chen, F. Multiwfn: A Multifunctional Wavefunction Analyzer. J. Comput. Chem. 2012, 33 (5), 580-592. 
(13) Grimme, S.; Hansen, A. A Practicable Real-Space Measure and Visualization of Static ElectronCorrelation Effects. Angew. Chemie Int. Ed. 2015, 54 (42), 12308-12313.

(14) Bauer, C. A.; Hansen, A.; Grimme, S. The Fractional Occupation Number Weighted Density as a Versatile Analysis Tool for Molecules with a Complicated Electronic Structure. Chem. - A Eur. J. 2017, 23 (25), 6150-6164.

(15) Neese, F. The ORCA Program System. Wiley Interdiscip. Rev. Comput. Mol. Sci. 2012, 2 (1), $73-78$.

(16) Neese, F. Software Update: The ORCA Program System, Version 4.0. Wiley Interdiscip. Rev. Comput. Mol. Sci. 2018, 8 (1), e1327. 


\section{Spectral appendix $\left({ }^{1} \mathrm{H},{ }^{13} \mathrm{C}\right.$ NMR, LDI, HRMS, UV, Fluo, HPLC).}

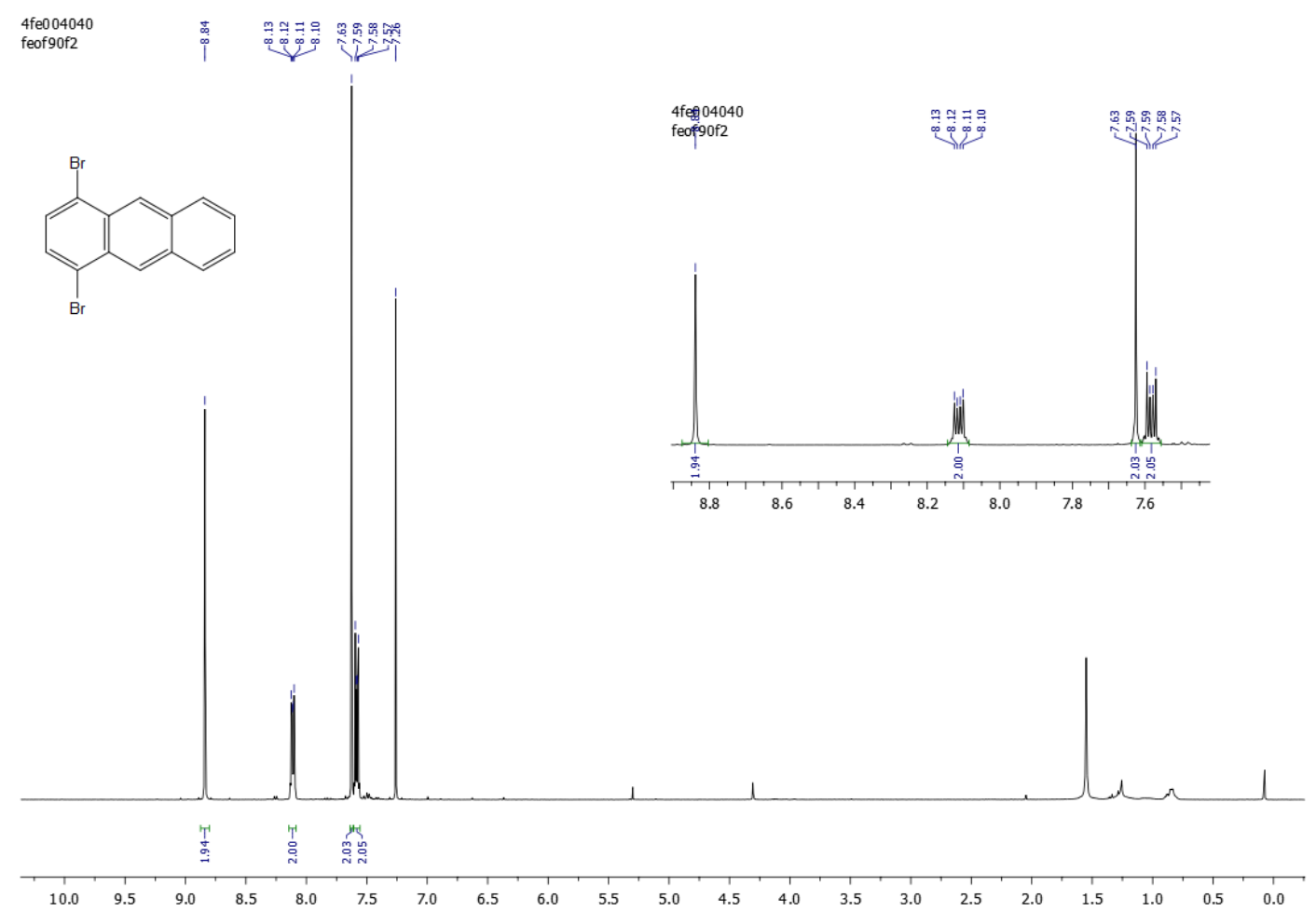

Figure S1. ${ }^{1} \mathrm{H}$ NMR (400 MHz, $\mathrm{CDCl}_{3}, 293 \mathrm{~K}$ ) spectrum of DHA-1.
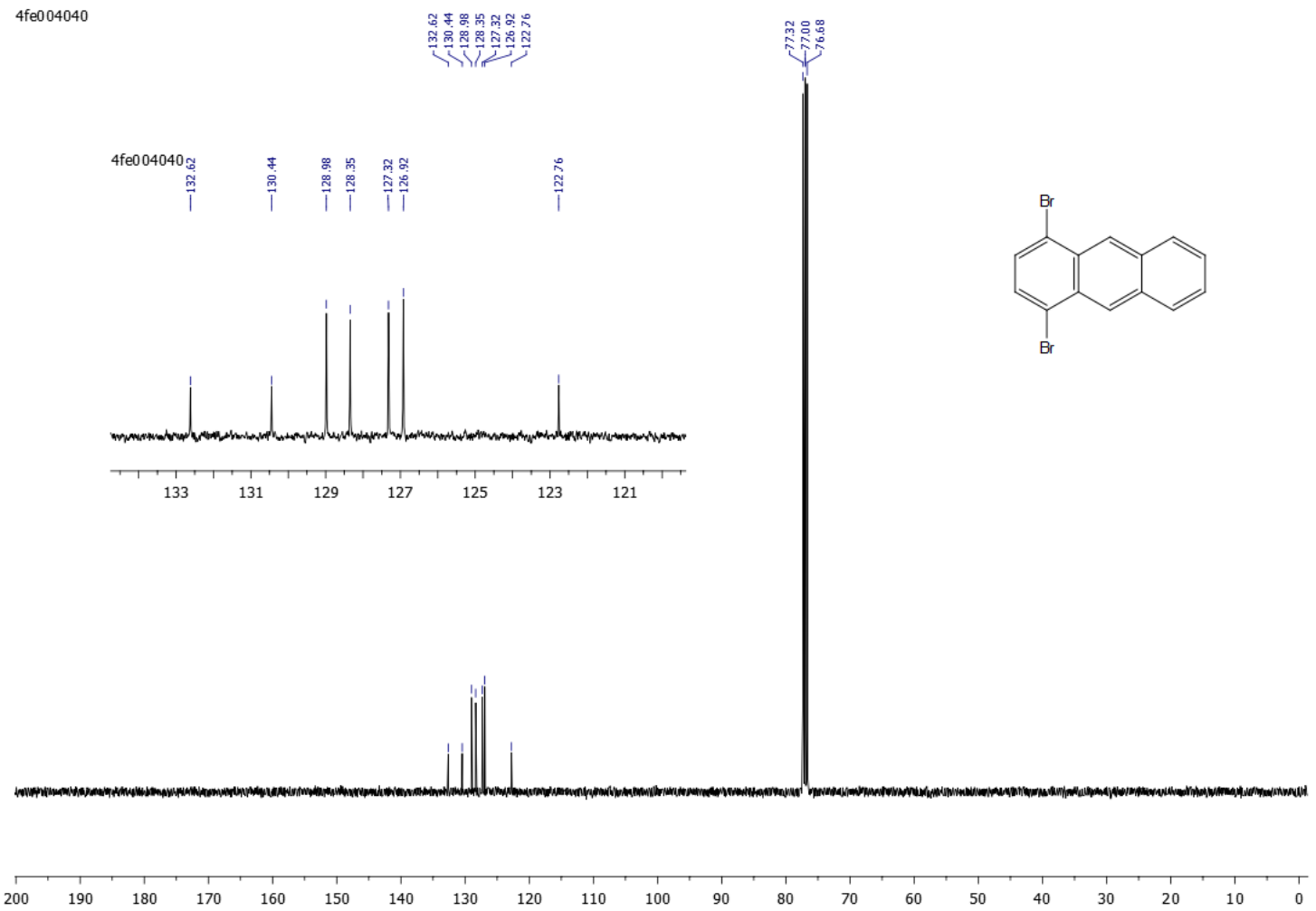

Figure S2. ${ }^{13} \mathrm{C}$ NMR (101 MHz, $\left.\mathrm{CDCl}_{3}, 293 \mathrm{~K}\right)$ spectrum of DHA-1. 
$4 \mathrm{fe} 005103$
$\mathrm{feof}-134$

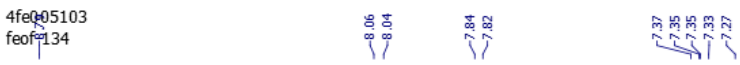
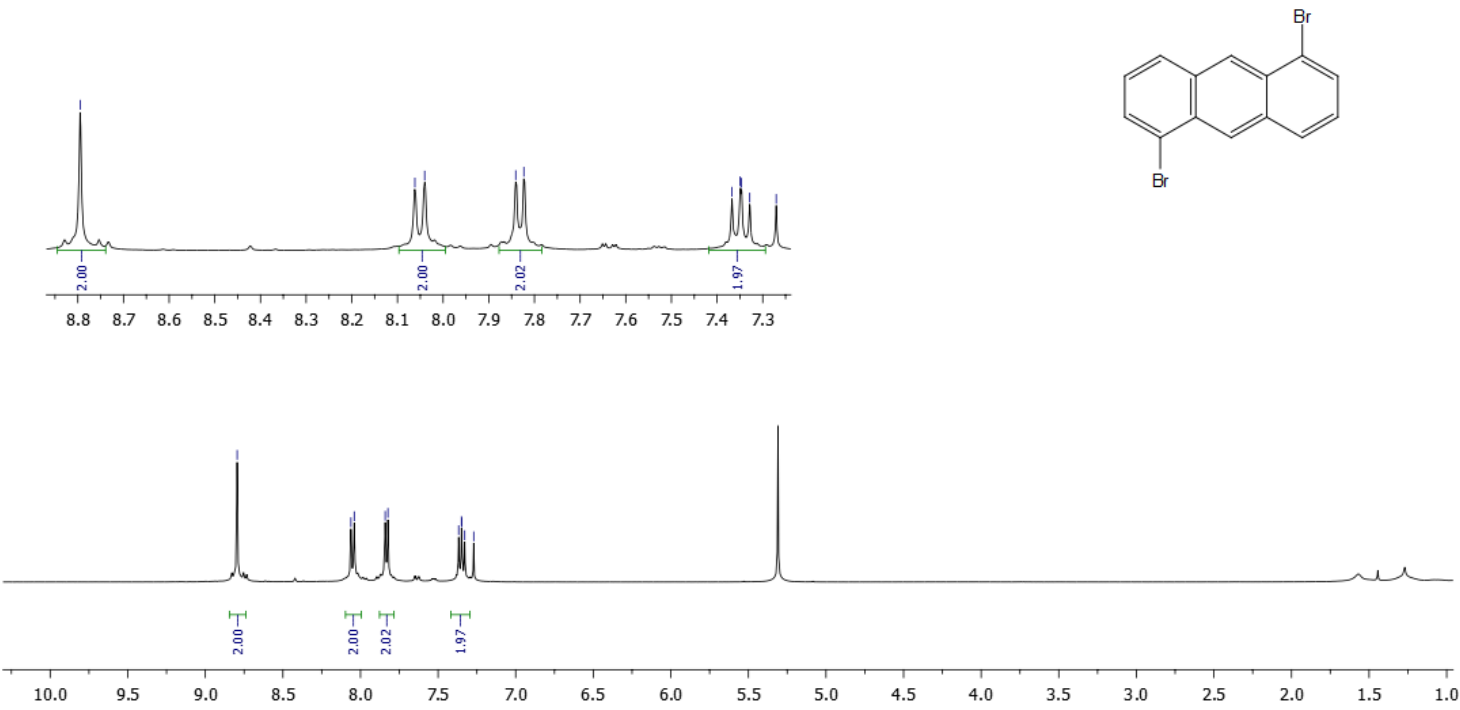

Figure S3. ${ }^{1} \mathrm{H}$ NMR (400 MHz, $\mathrm{CDCl}_{3}, 293 \mathrm{~K}$ ) spectrum of DHA-2.
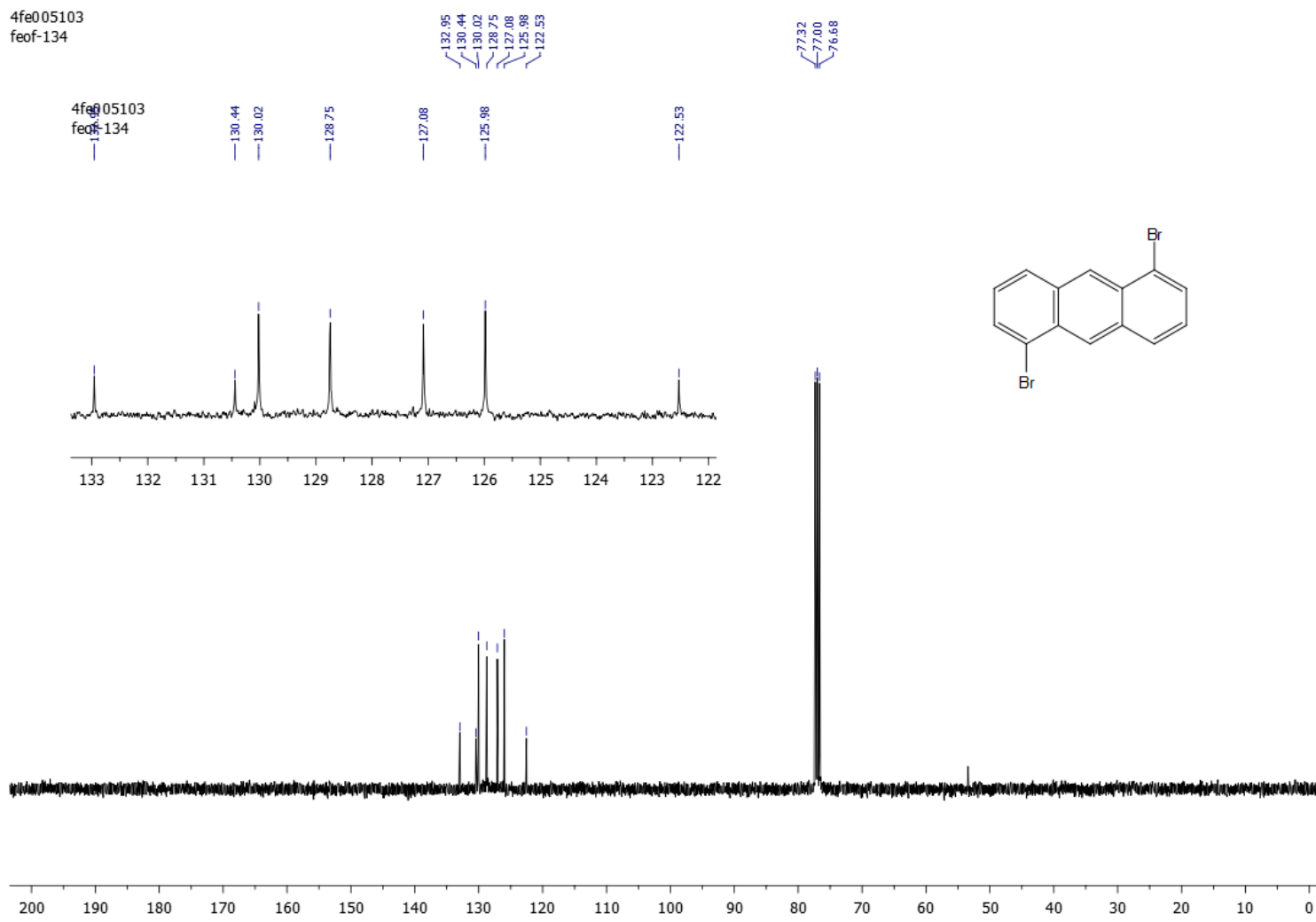

Figure S4. ${ }^{13} \mathrm{C}$ NMR (101 MHz, $\left.\mathrm{CDCl}_{3}, 293 \mathrm{~K}\right)$ spectrum of DHA-2. 


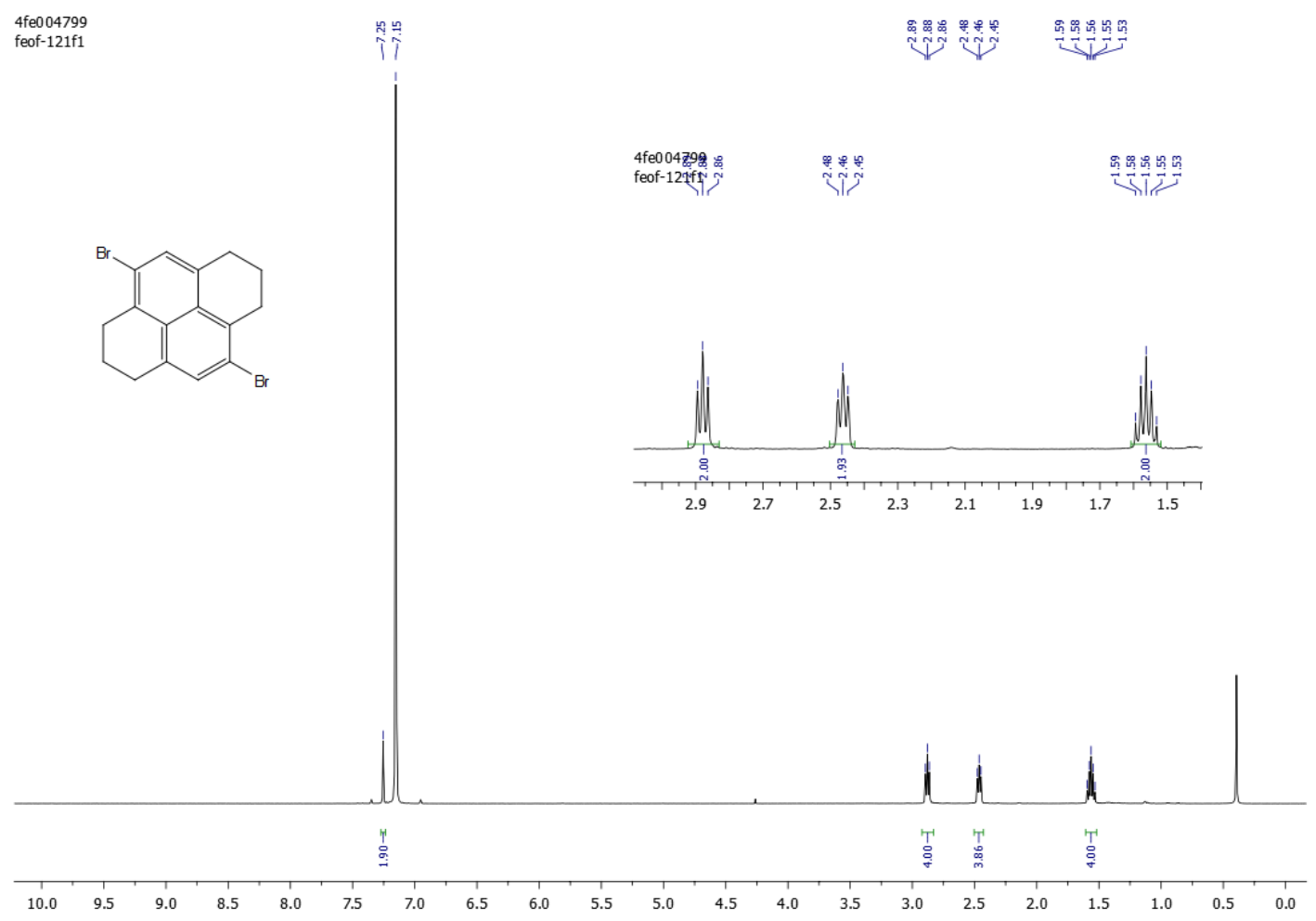

Figure S5. ${ }^{1} \mathrm{H}$ NMR (400 MHz, C6 $\left.\mathrm{D}_{6}, 293 \mathrm{~K}\right)$ spectrum of 4,9-dibromo-1,2,3,6,7,9-hexahydropyrene.
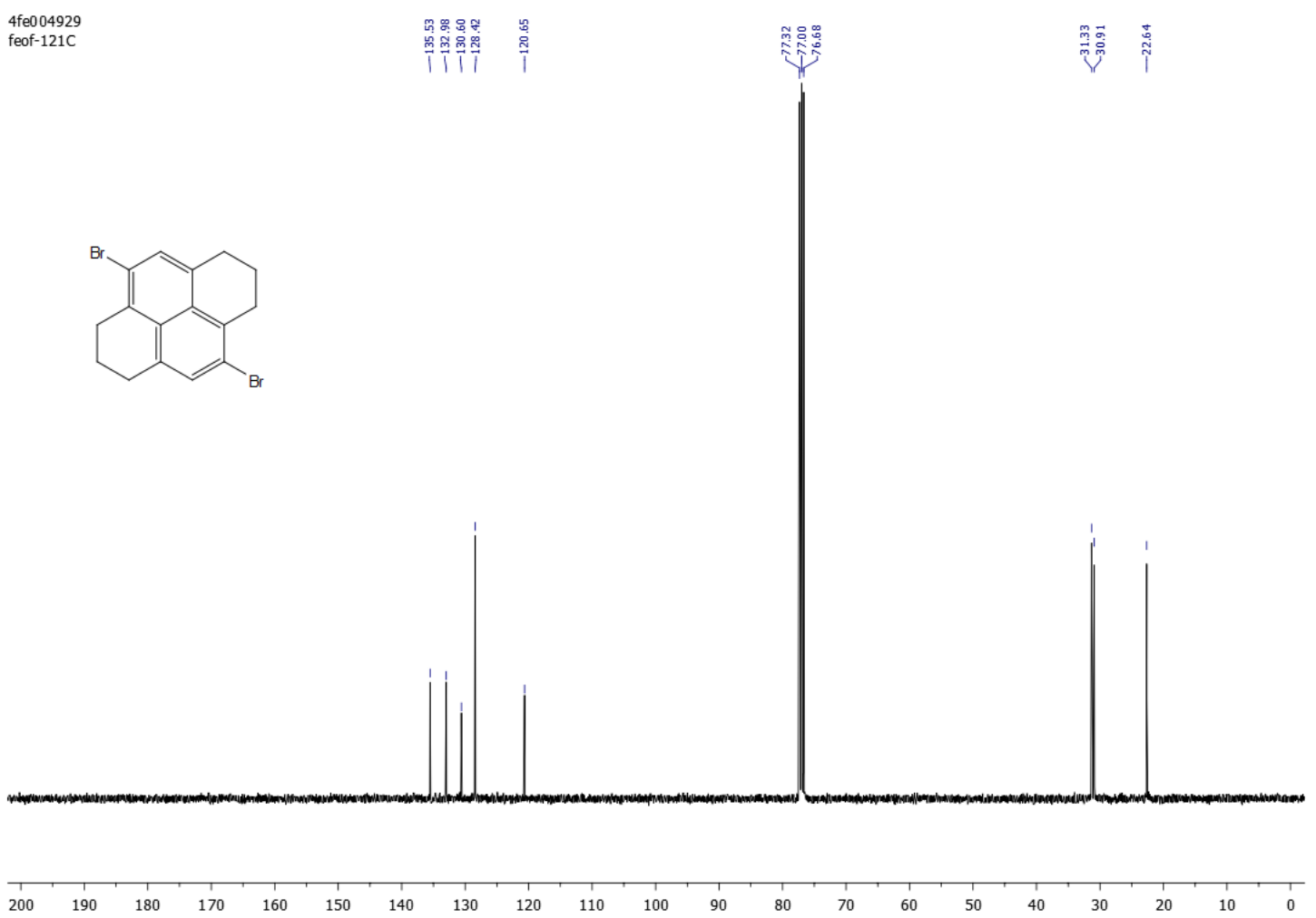

Figure S6. ${ }^{13} \mathrm{C} \mathrm{NMR}\left(101 \mathrm{MHz}, \mathrm{CDCl}_{3}, 293 \mathrm{~K}\right)$ spectrum of 4,9-dibromo-1,2,3,6,7,9hexahydropyrene. 

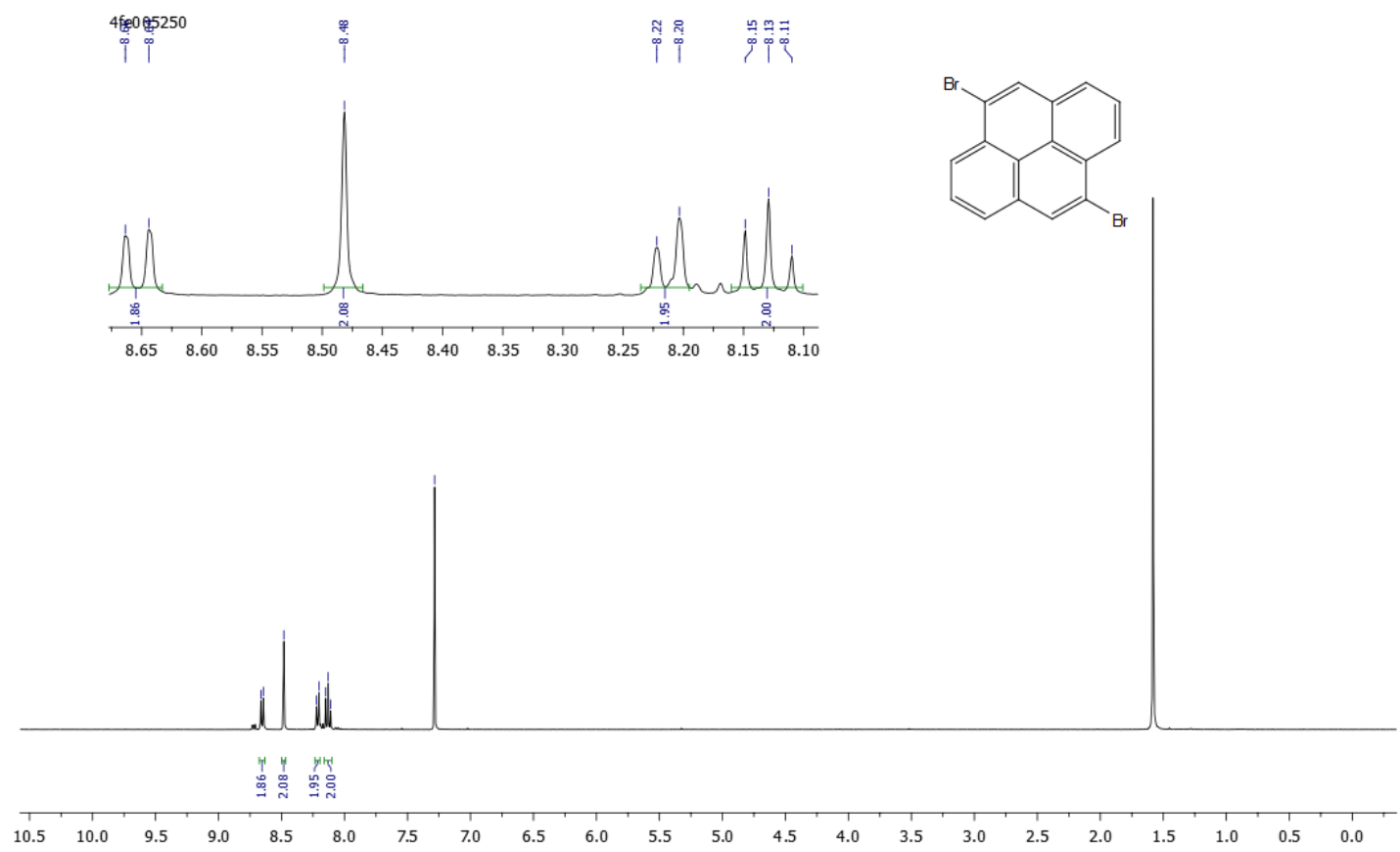

Figure S7. ${ }^{1} \mathrm{H}$ NMR (400 MHz, $\left.\mathrm{C}_{6} \mathrm{D}_{6}, 293 \mathrm{~K}\right)$ spectrum of DHA-4.

$4 \mathrm{fe} 005250$

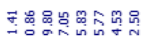

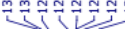

象是:

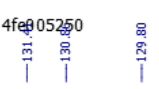

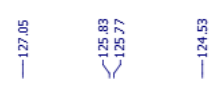

กับ
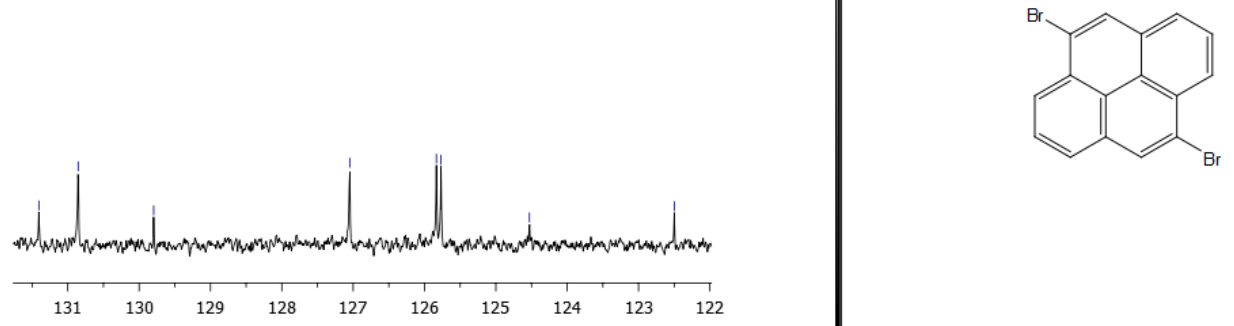

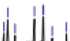

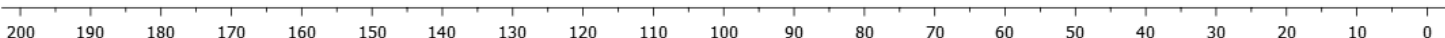

Figure S8. ${ }^{13} \mathrm{C}$ NMR (101 MHz, $\left.\mathrm{CDCl}_{3}, 293 \mathrm{~K}\right)$ spectrum of DHA-4. 


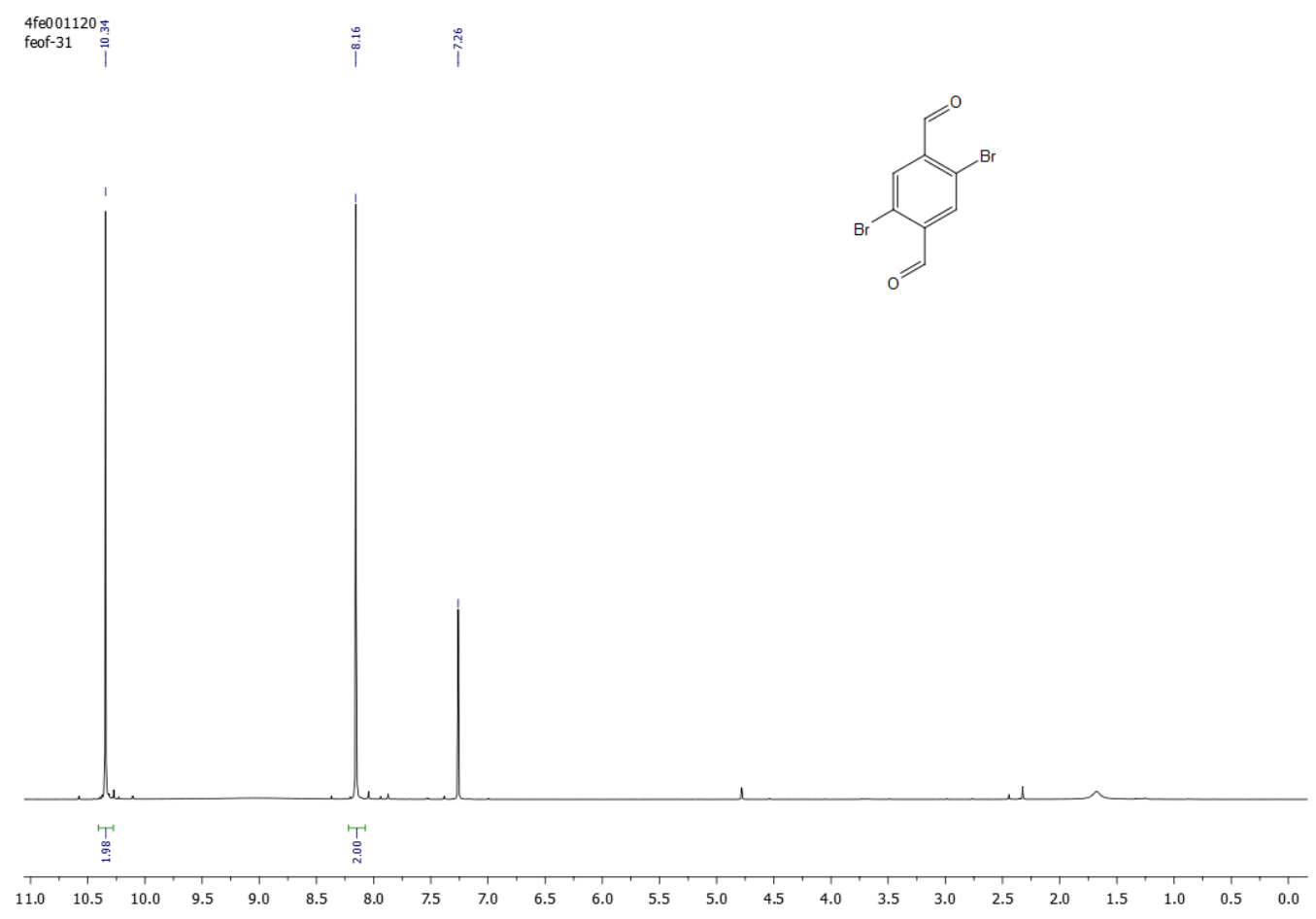

Figure S9. ${ }^{1} \mathrm{H} \mathrm{NMR}\left(400 \mathrm{MHz}, \mathrm{CDCl}_{3}, 293 \mathrm{~K}\right)$ spectrum of BS-1.
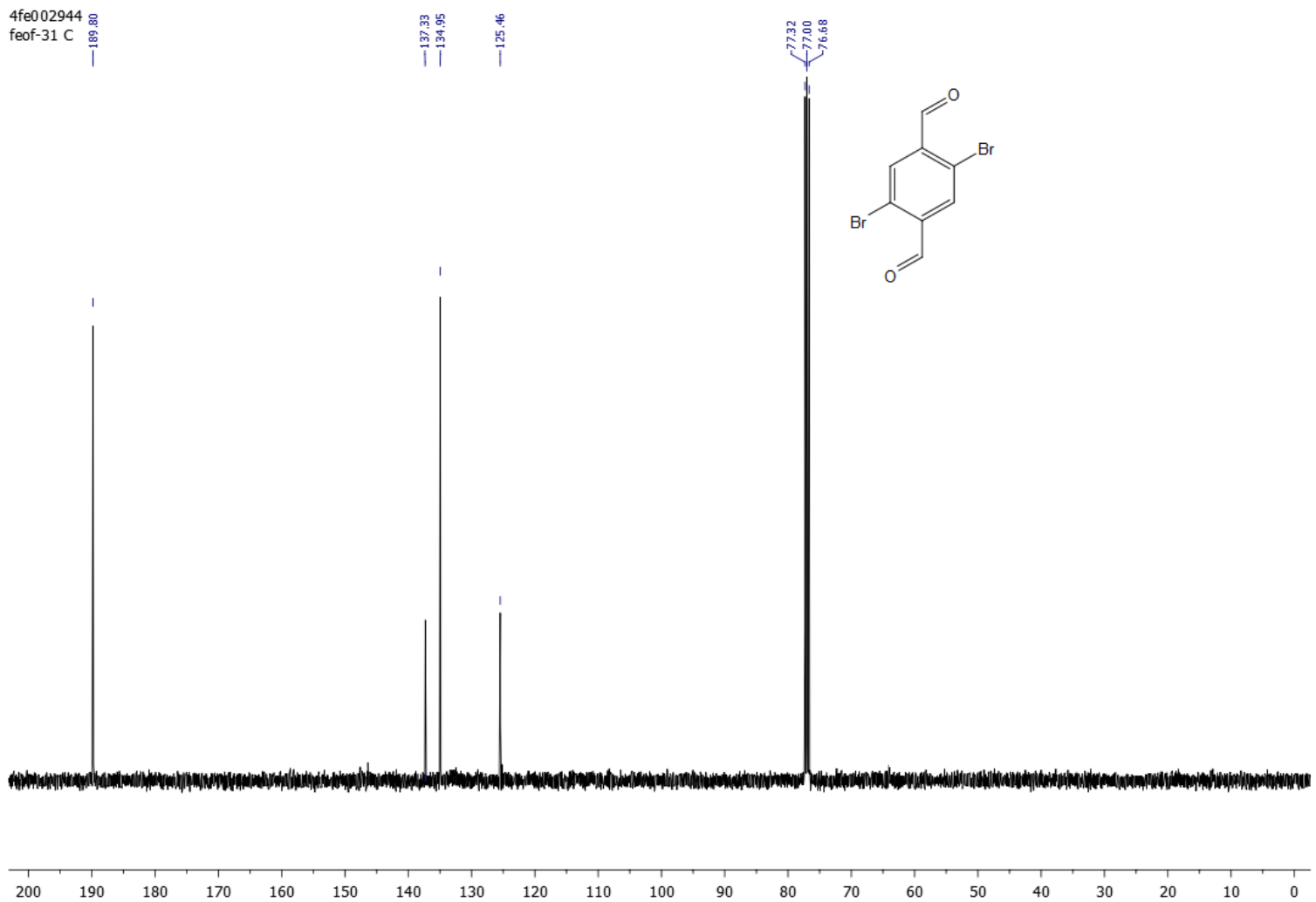

Figure S10. ${ }^{13} \mathrm{C}$ NMR $\left(101 \mathrm{MHz}, \mathrm{CDCl}_{3}, 293 \mathrm{~K}\right)$ spectrum of BS-1. 

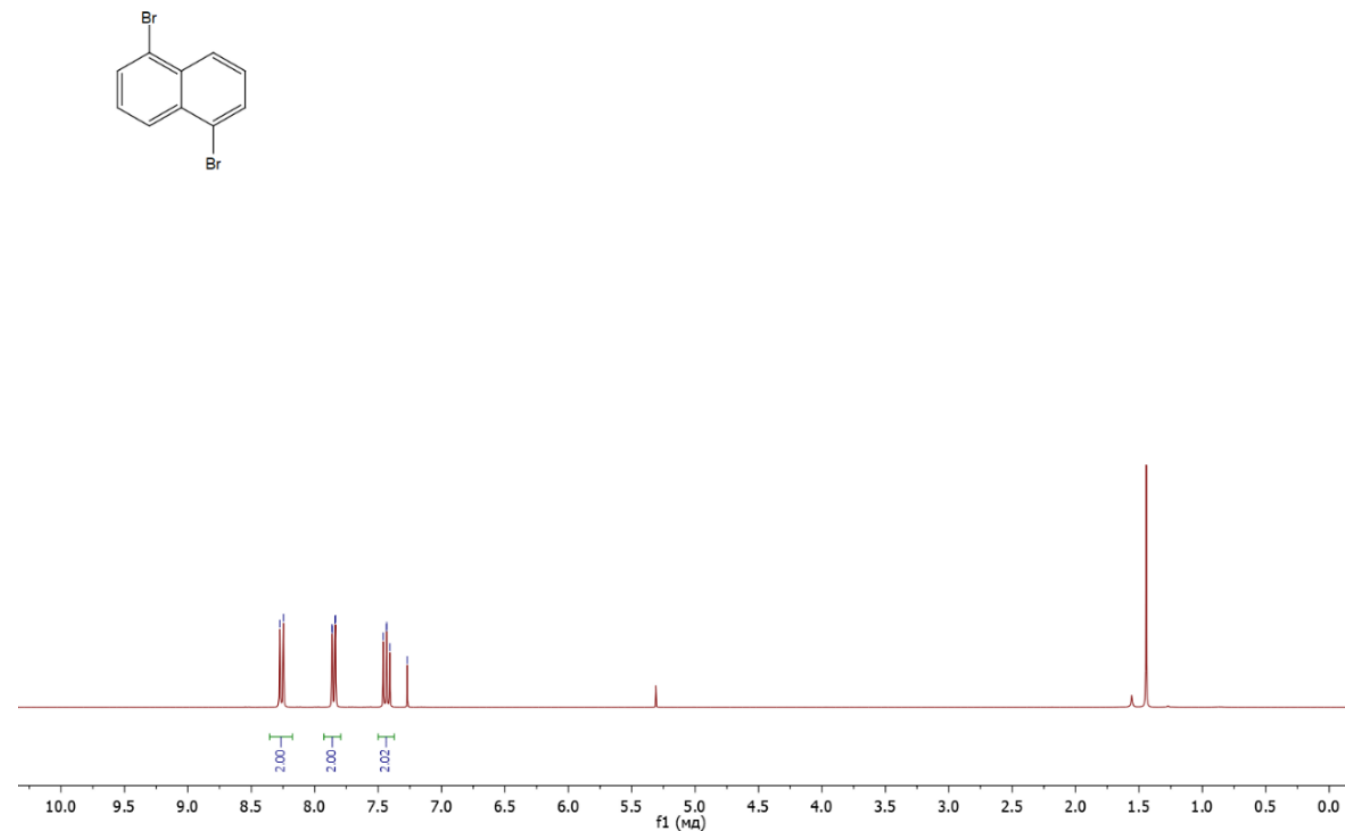

Figure S11. ${ }^{1} \mathrm{H}$ NMR (400 MHz, $\mathrm{CDCl}_{3}, 293 \mathrm{~K}$ ) spectrum of DHA-5.
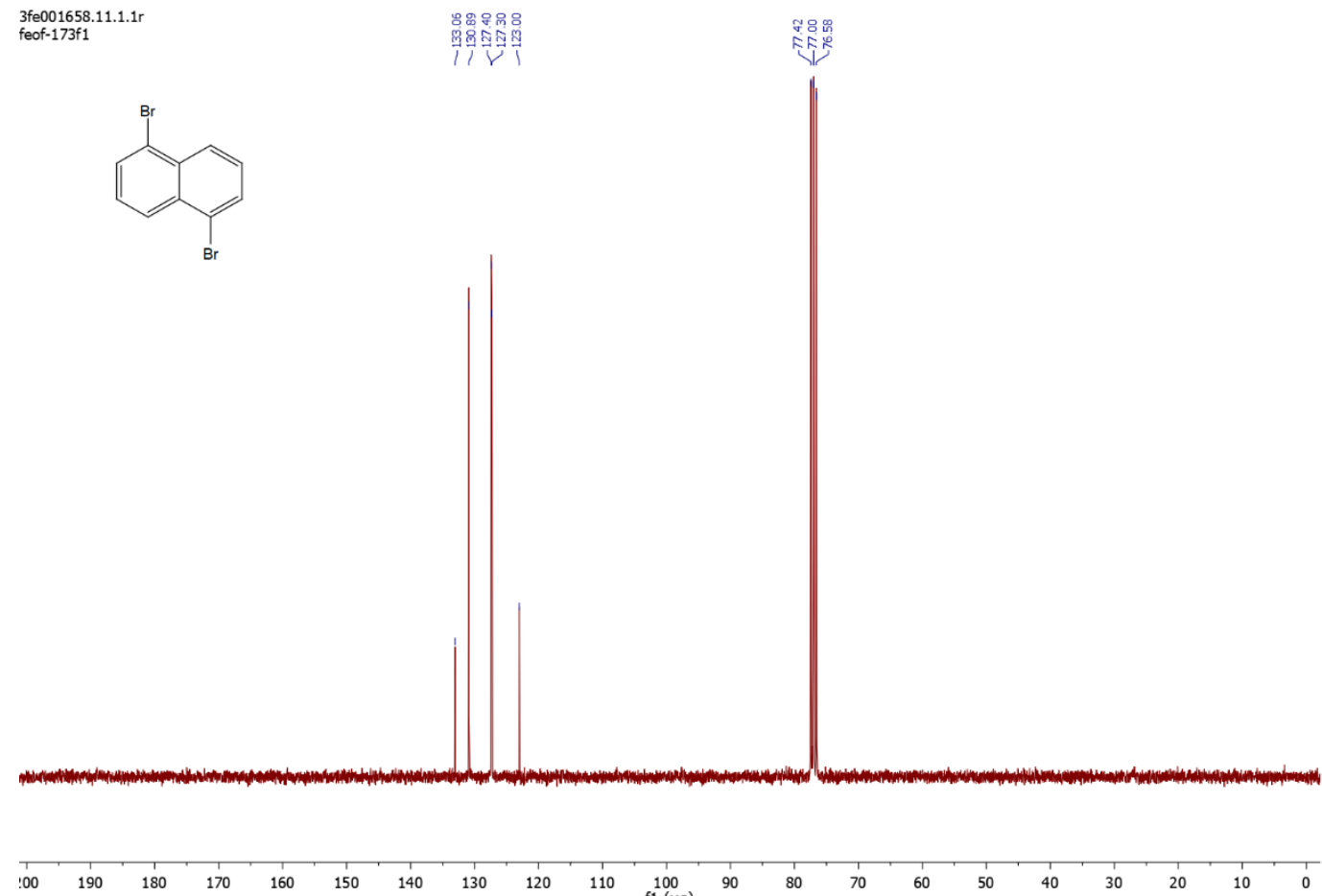

Figure S12. ${ }^{13} \mathrm{C}$ NMR $\left(101 \mathrm{MHz}, \mathrm{CDCl}_{3}, 293 \mathrm{~K}\right)$ spectrum of DHA-5. 

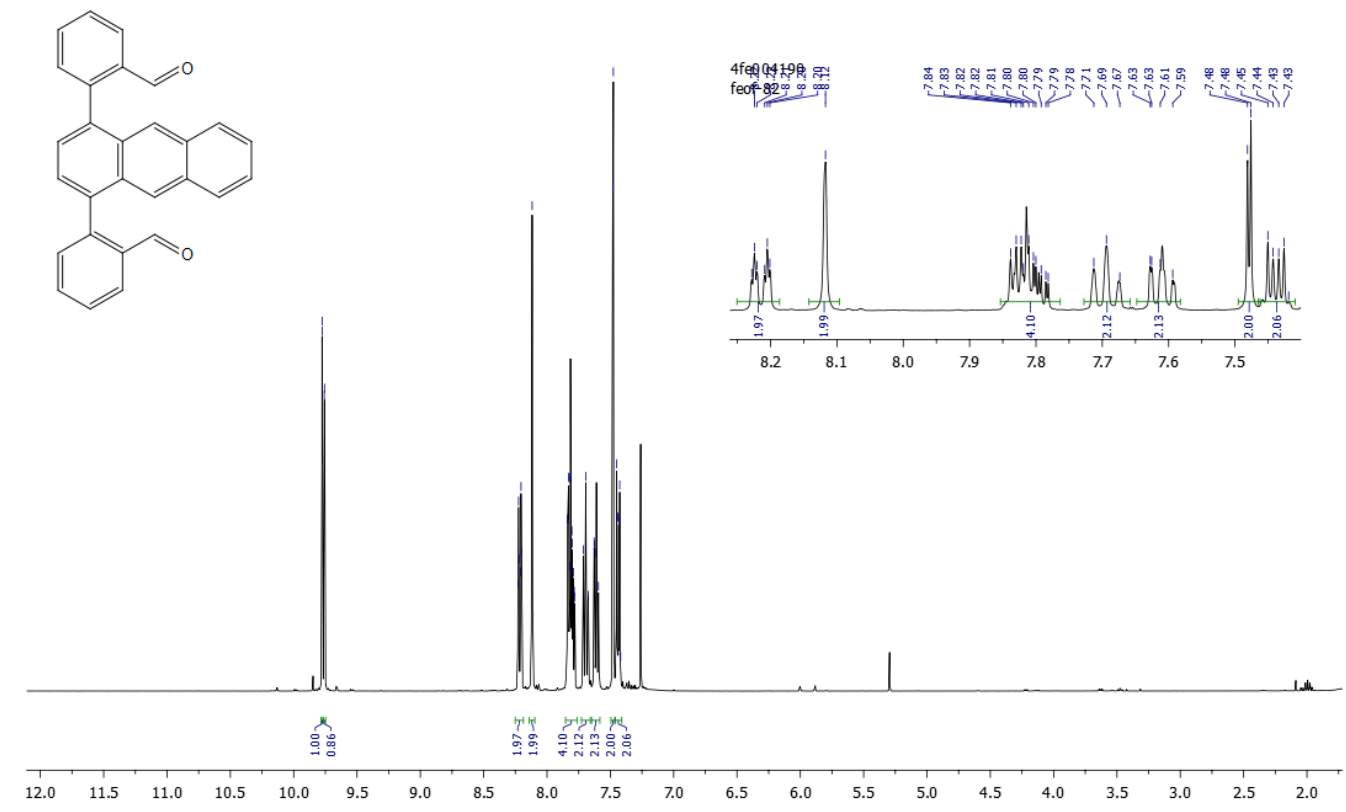

Figure S13. ${ }^{1} \mathrm{H} \mathrm{NMR}\left(400 \mathrm{MHz}, \mathrm{CDCl}_{3}, 293 \mathrm{~K}\right)$ spectrum of P1.
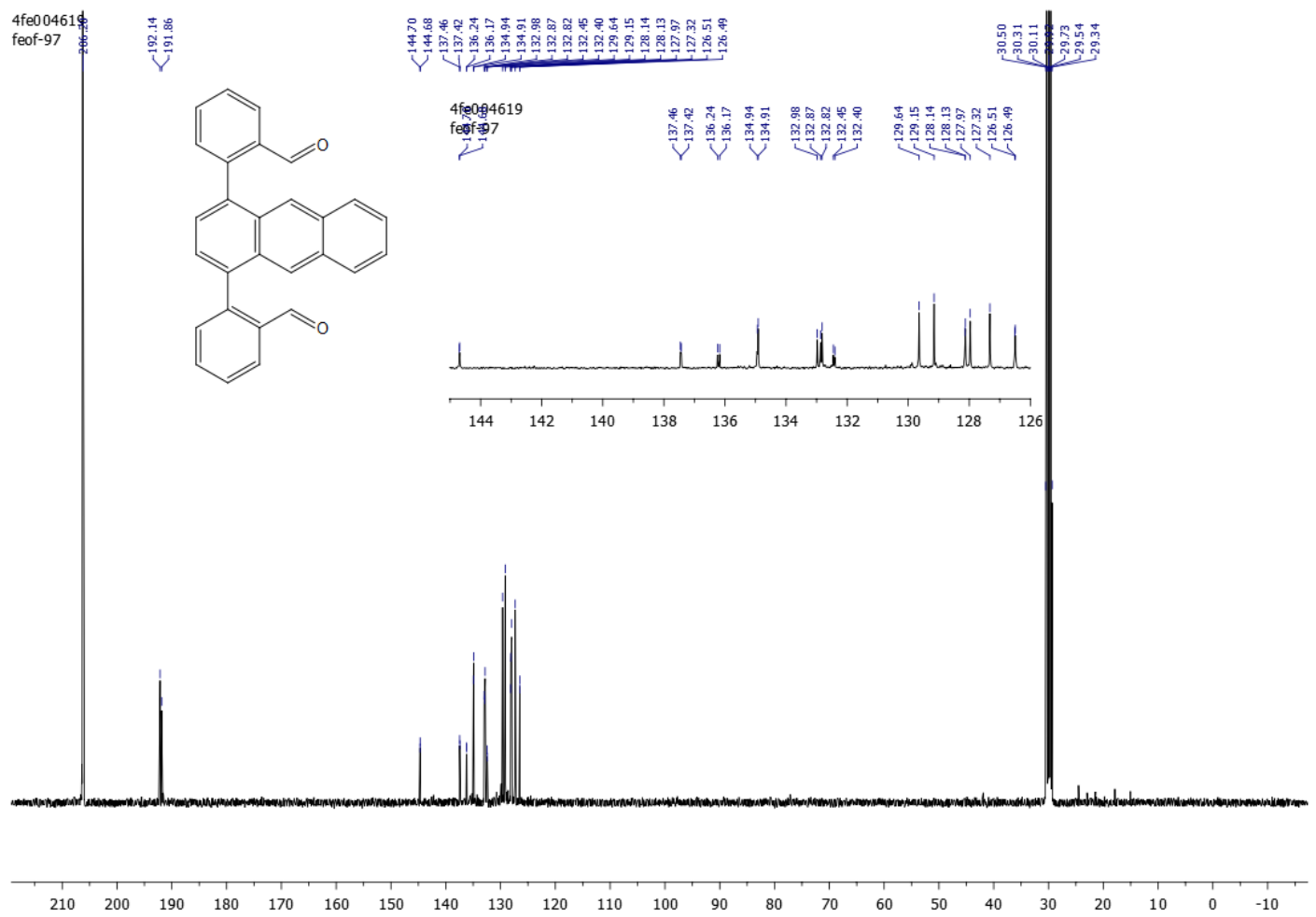

Figure S14. ${ }^{13} \mathrm{C}$ NMR (101 MHz, Acetone-d $6,293 \mathrm{~K}$ ) spectrum of P1. 


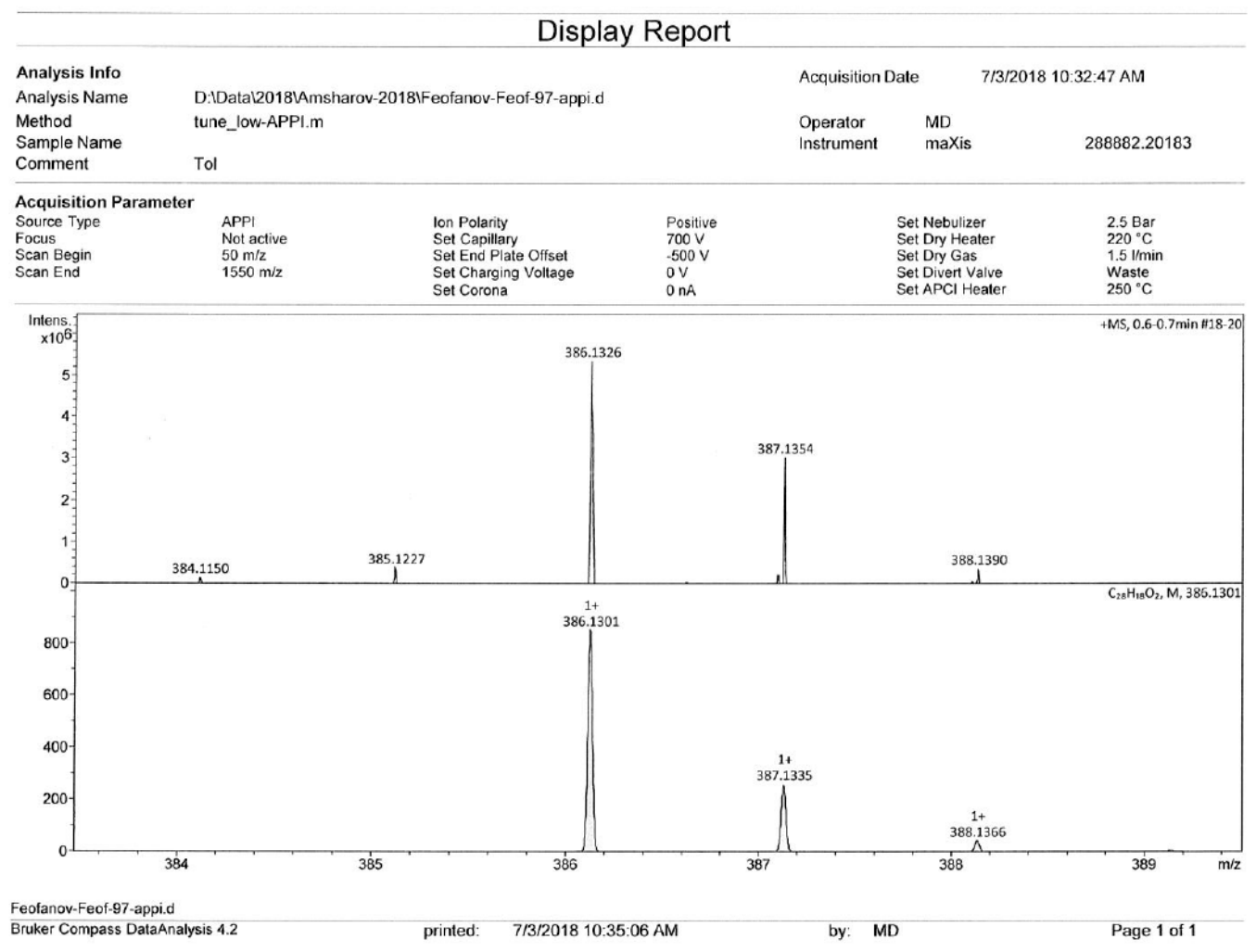

Figure S15. APPI-HRMS spectrum of P1.

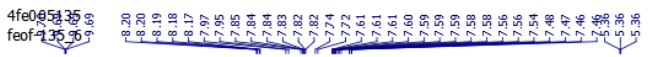
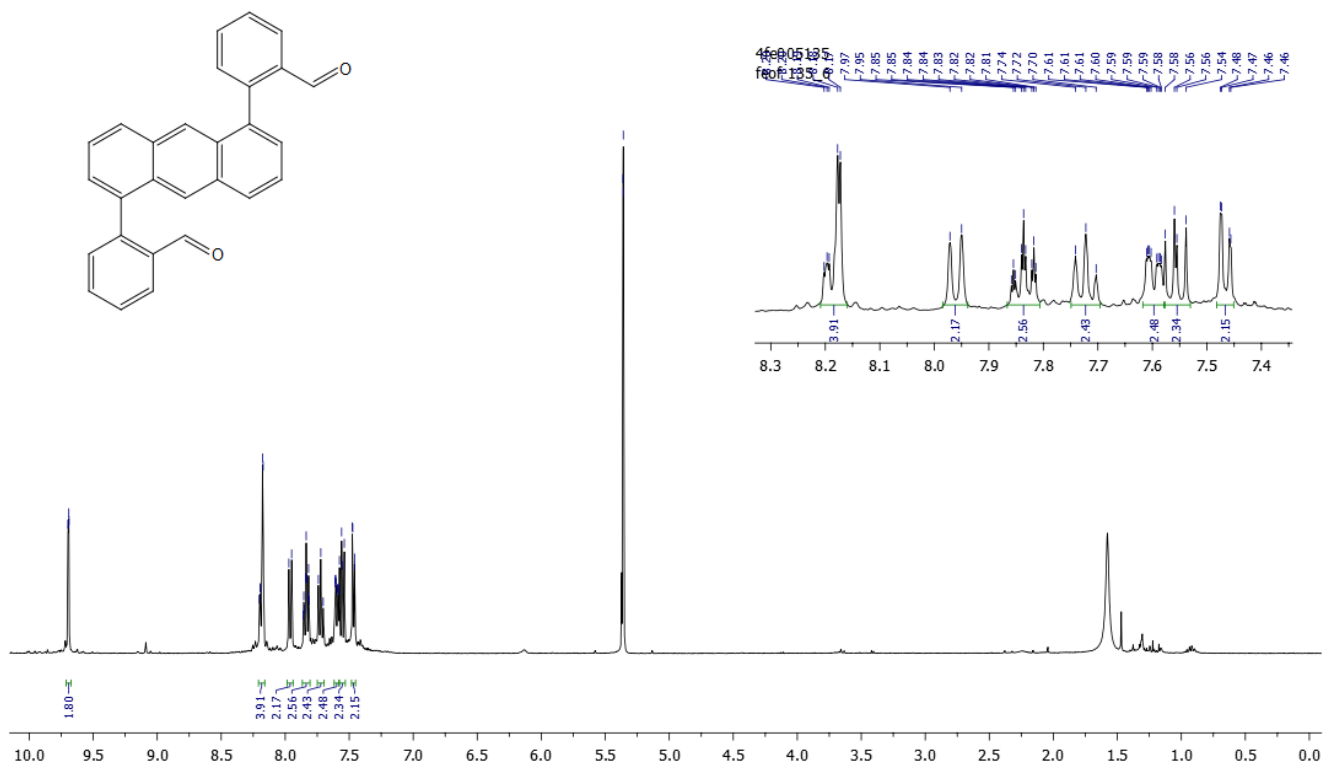

Figure S16. ${ }^{1} \mathrm{H} \mathrm{NMR}\left(400 \mathrm{MHz}, \mathrm{CD}_{2} \mathrm{Cl}_{2}, 293 \mathrm{~K}\right.$ ) spectrum of $\mathbf{P 2}$. 

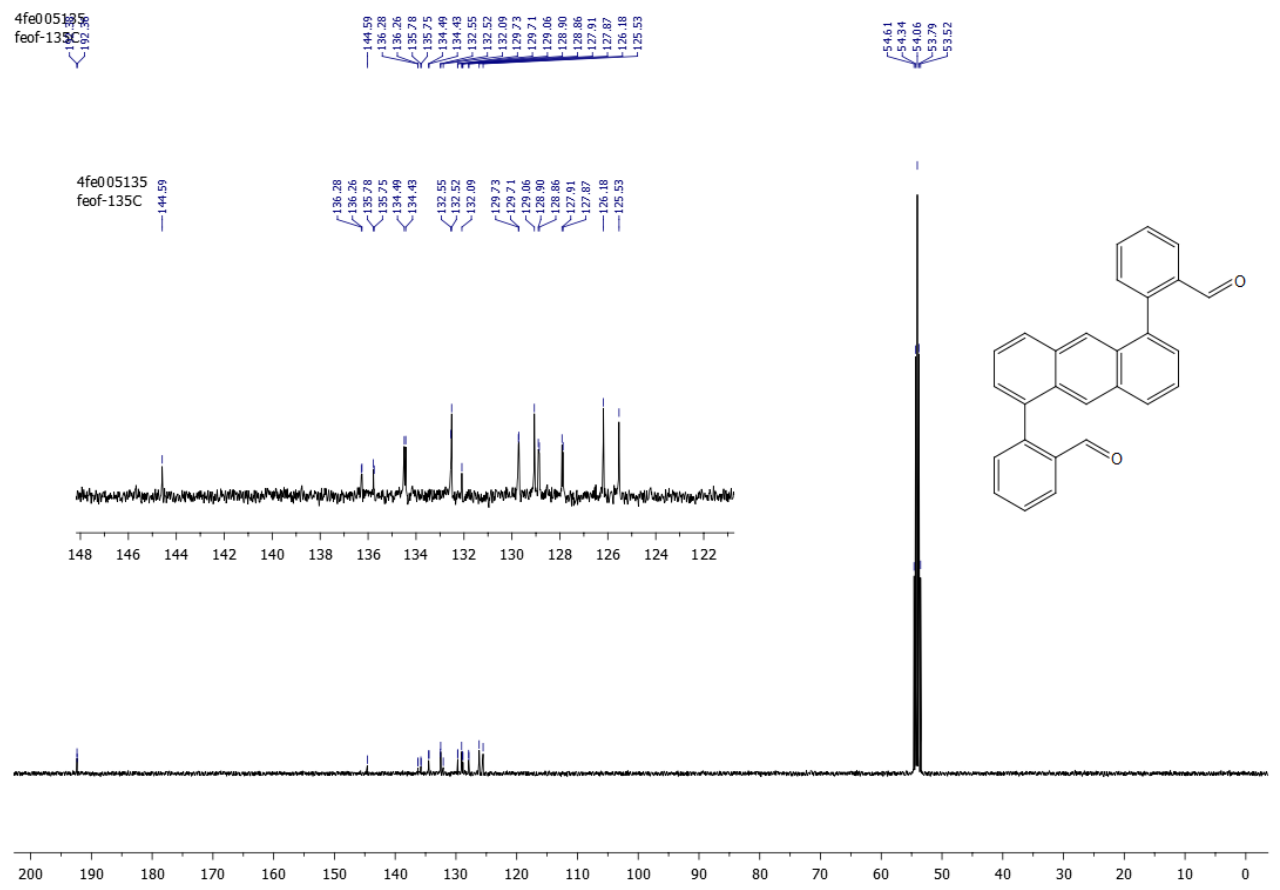

Figure S17. ${ }^{13} \mathrm{C}$ NMR (101 MHz, $\left.\mathrm{CD}_{2} \mathrm{Cl}_{2}, 293 \mathrm{~K}\right)$ spectrum of $\mathbf{P 2}$.

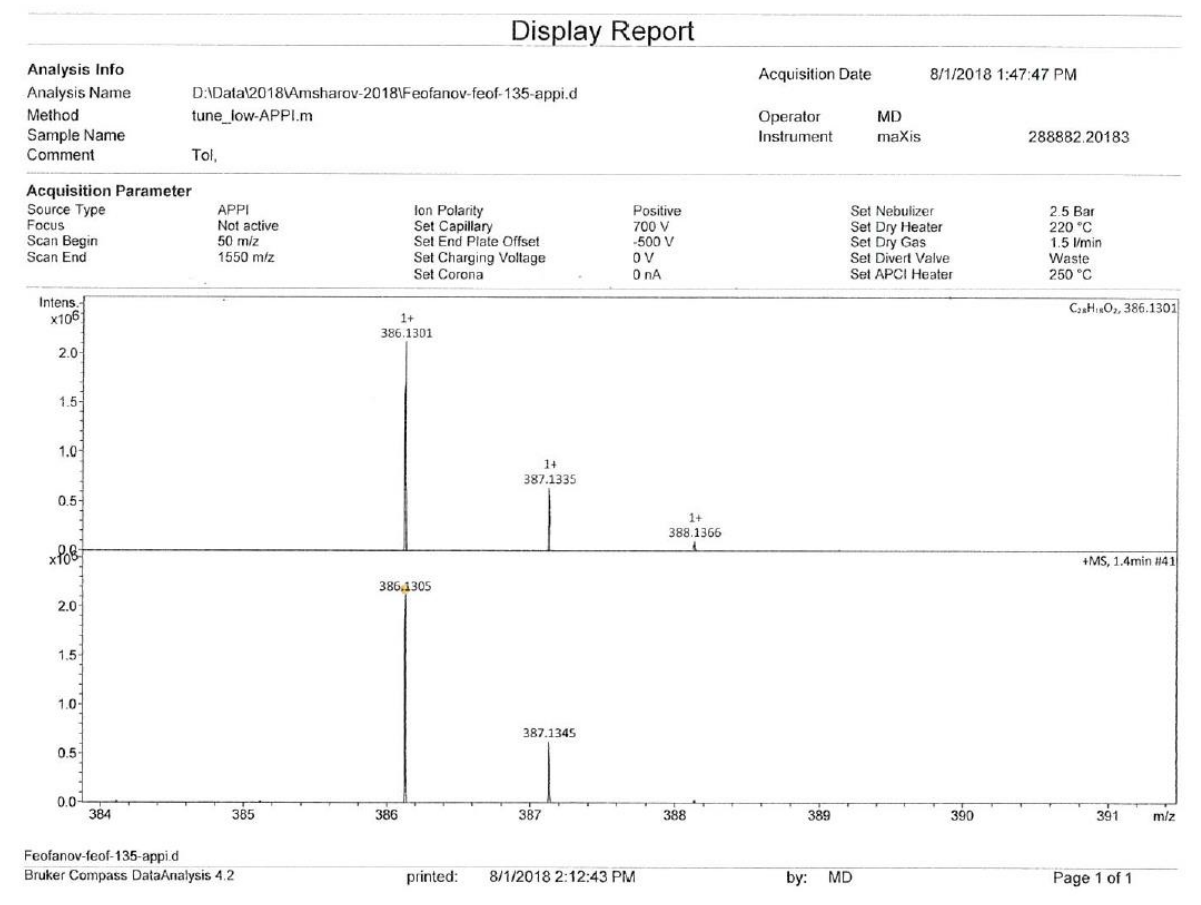

Figure S18. APPI-HRMS spectrum of $\mathbf{P 2}$. 


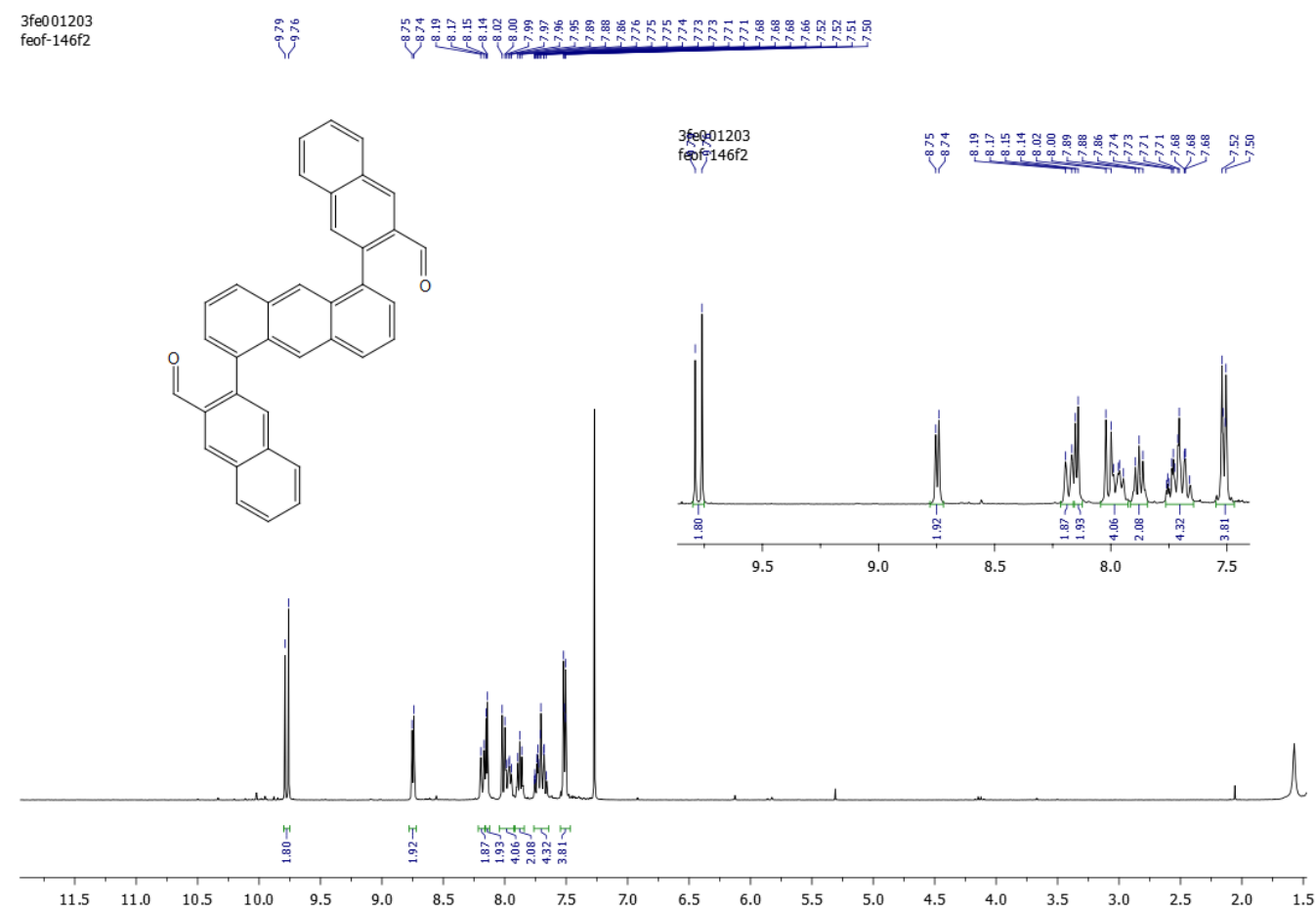

Figure S19. ${ }^{1} \mathrm{H} \mathrm{NMR}\left(400 \mathrm{MHz}, \mathrm{CDCl}_{3}, 293 \mathrm{~K}\right)$ spectrum of $\mathbf{P 3}$.
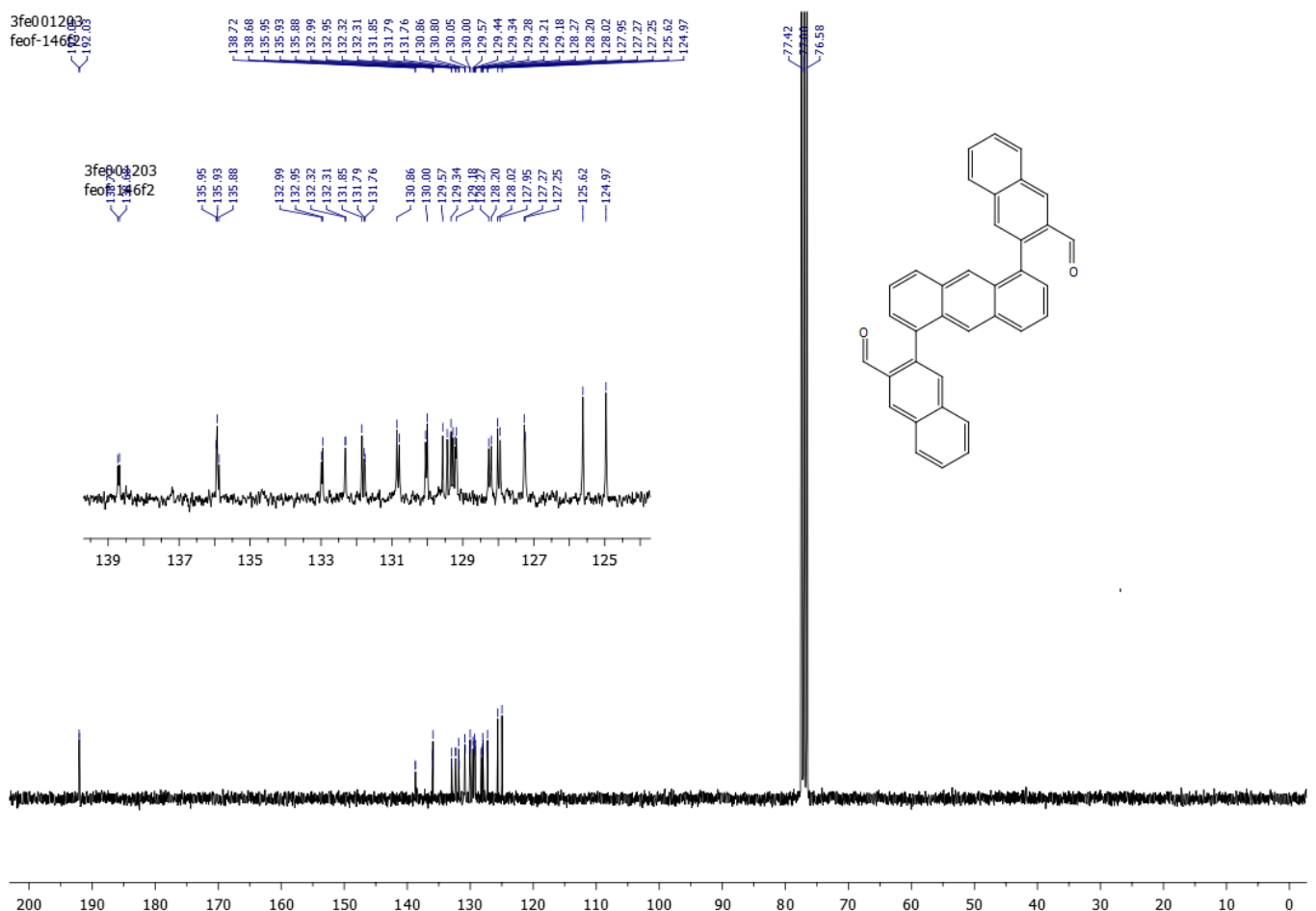

Figure S20. ${ }^{13} \mathrm{C}$ NMR (101 MHz, $\left.\mathrm{CDCl}_{3}, 293 \mathrm{~K}\right)$ spectrum of $\mathbf{P 3}$. 
Display Report

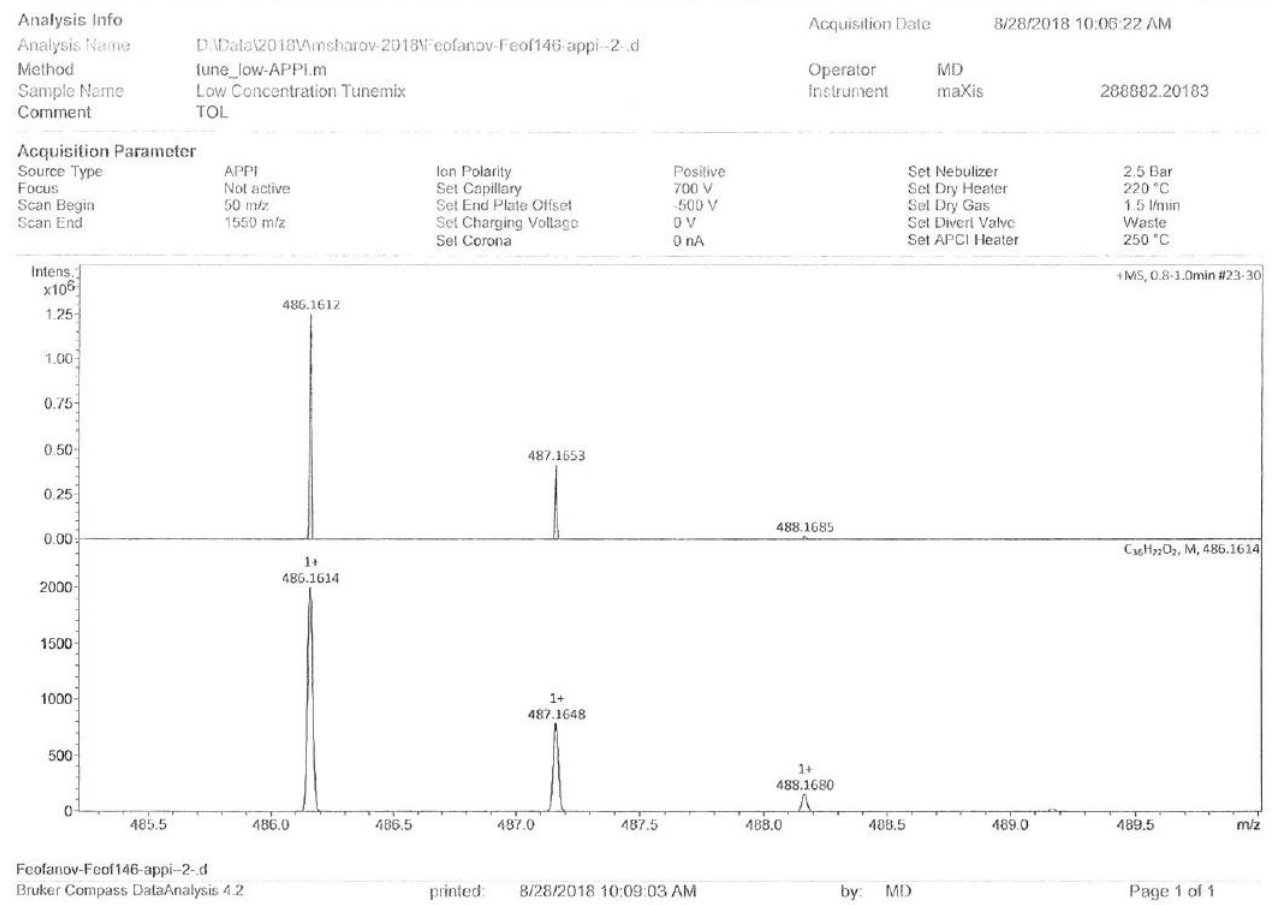

Figure S21. APPI-HRMS spectrum of P3.
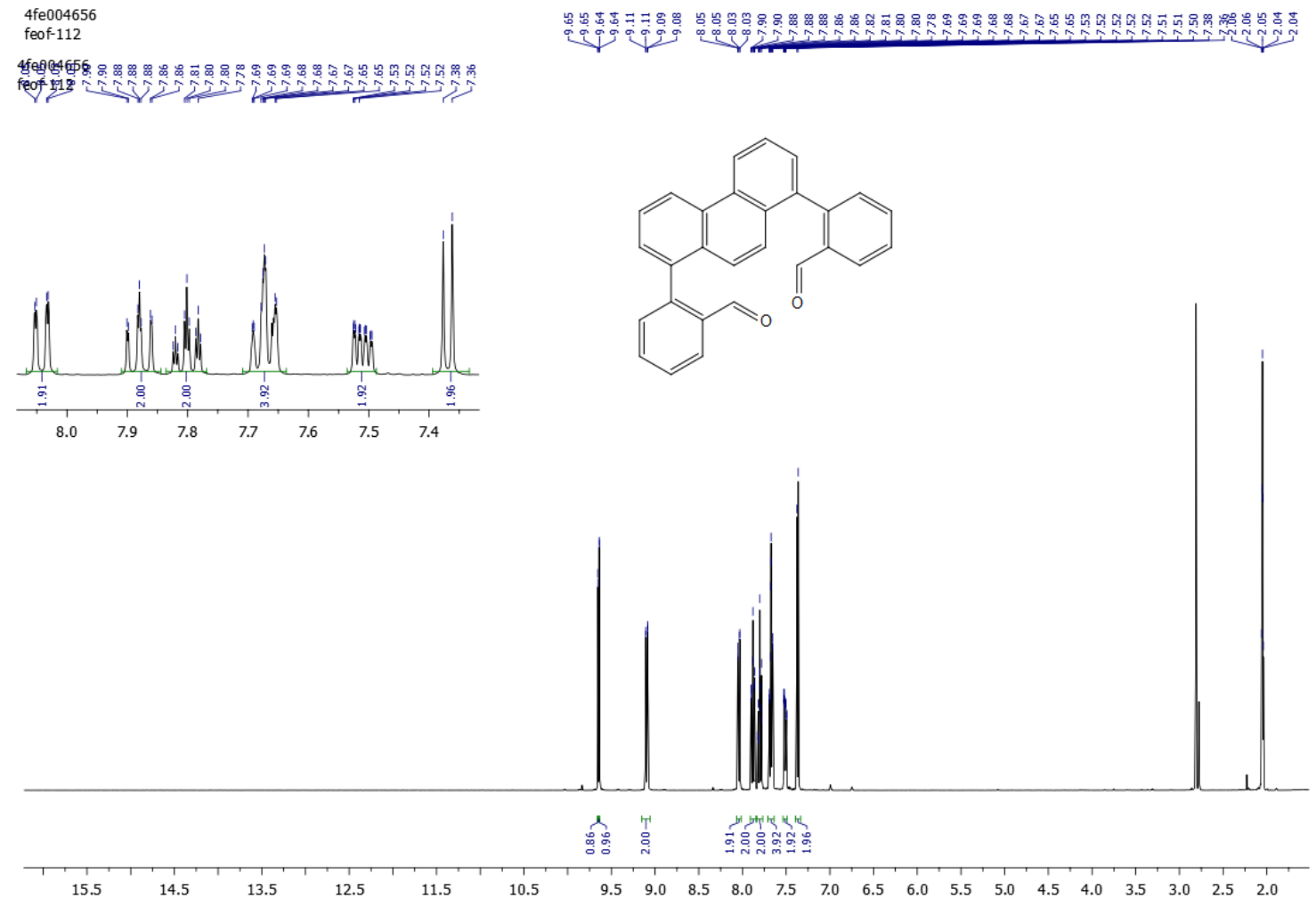

Figure S22. ${ }^{1} \mathrm{H}$ NMR (400 MHz, Acetone-d 6 , $293 \mathrm{~K}$ ) spectrum of $\mathbf{P 4 .}$ 


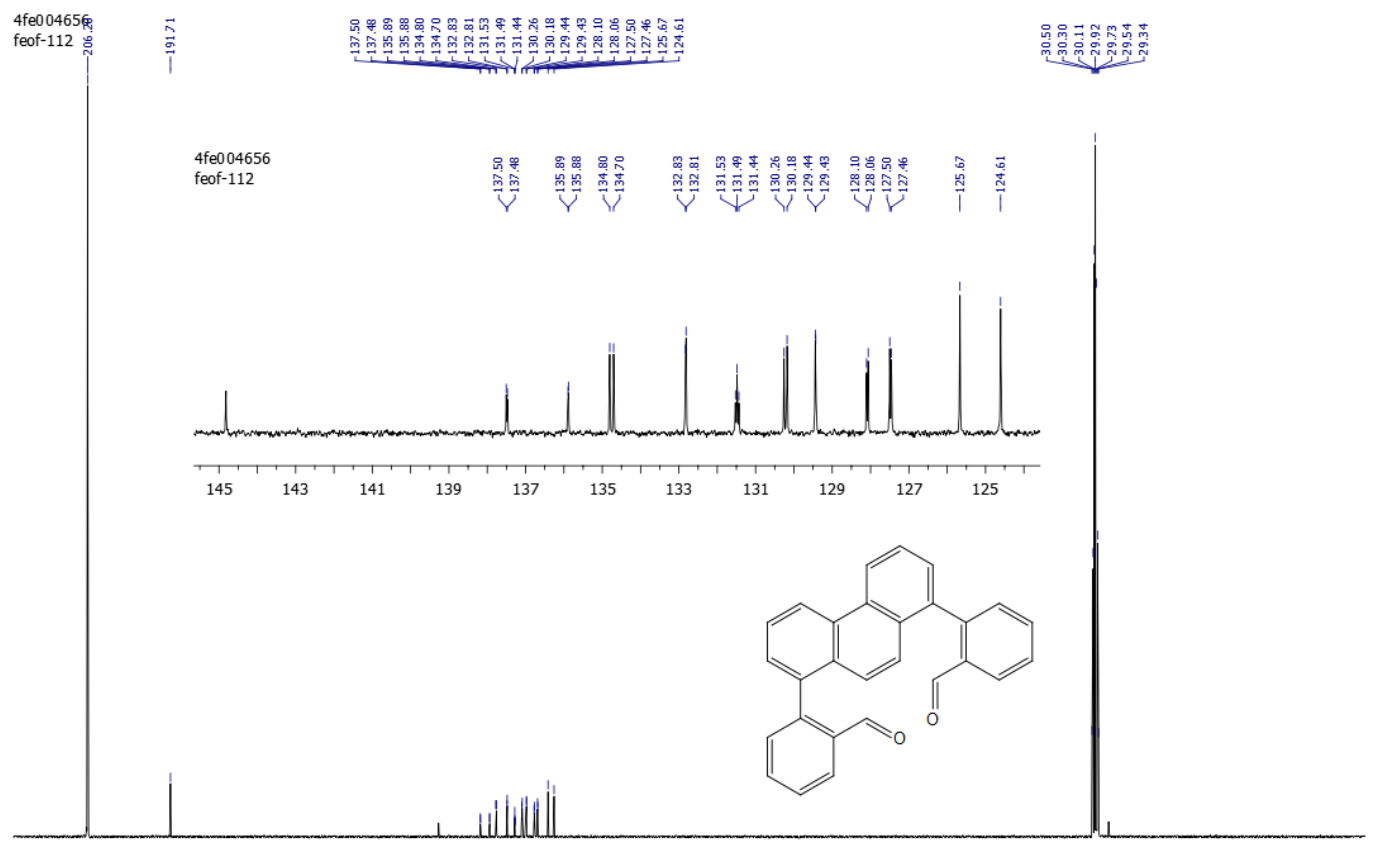

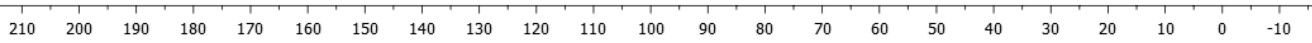

Figure S23. ${ }^{13} \mathrm{C}$ NMR (101 MHz, Acetone-d 6 , $\left.293 \mathrm{~K}\right)$ spectrum of P4.

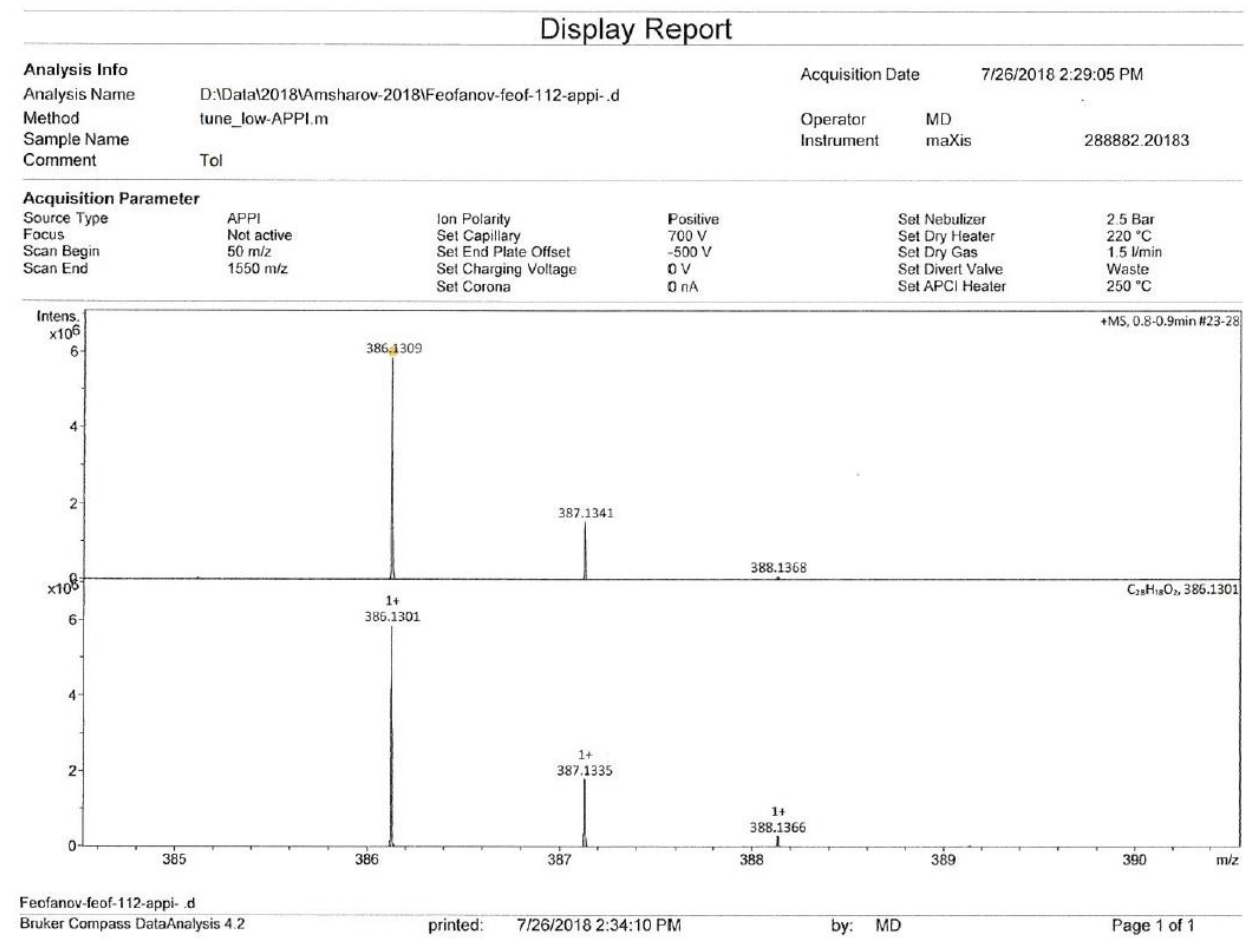

Figure S24. APPI-HRMS spectrum of P4. 


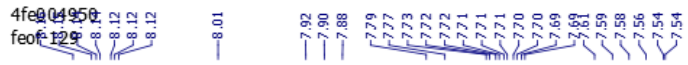
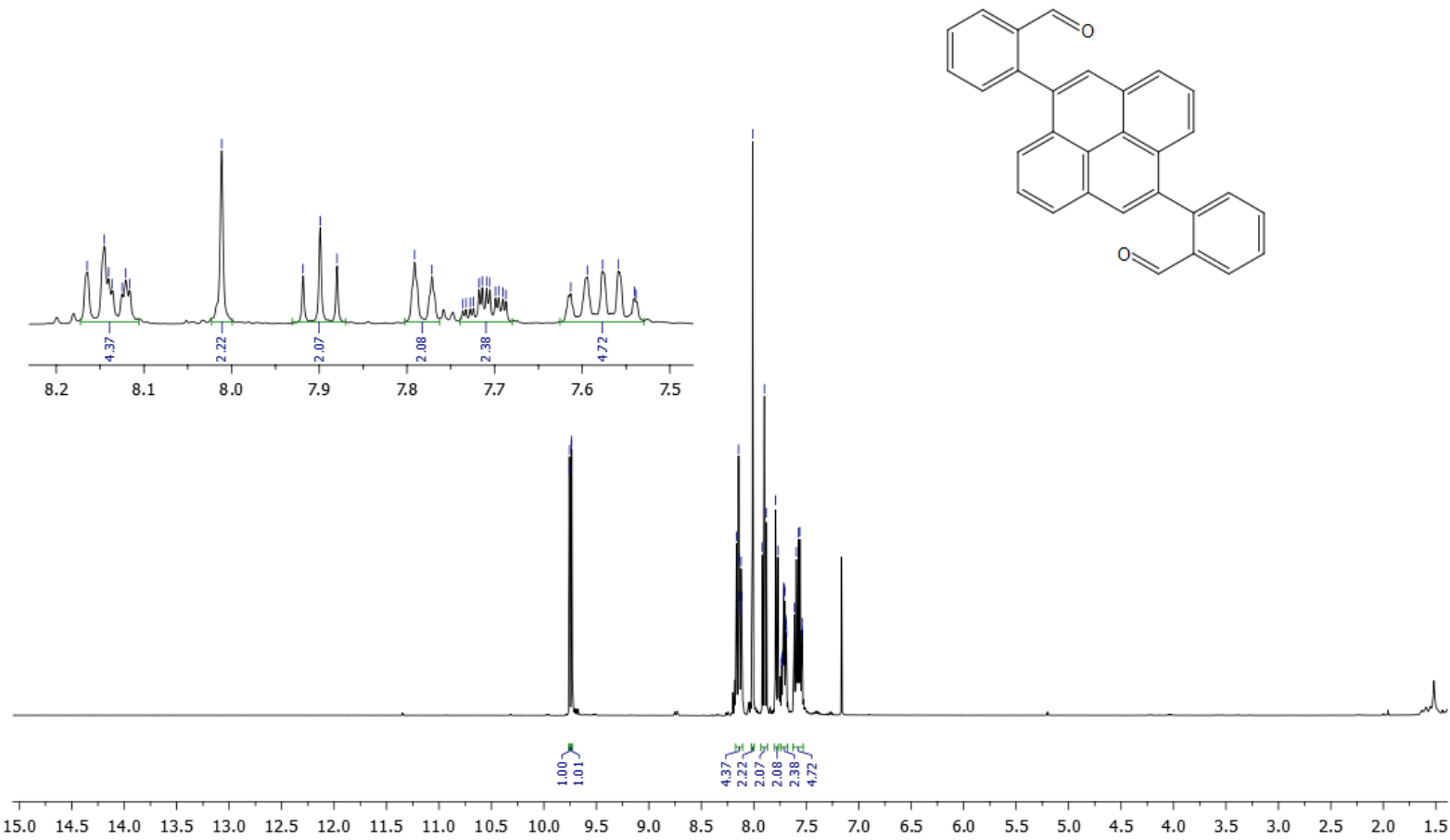

Figure S25. ${ }^{1} \mathrm{H} \mathrm{NMR}\left(400 \mathrm{MHz}, \mathrm{CDCl}_{3}, 293 \mathrm{~K}\right)$ spectrum of P5.

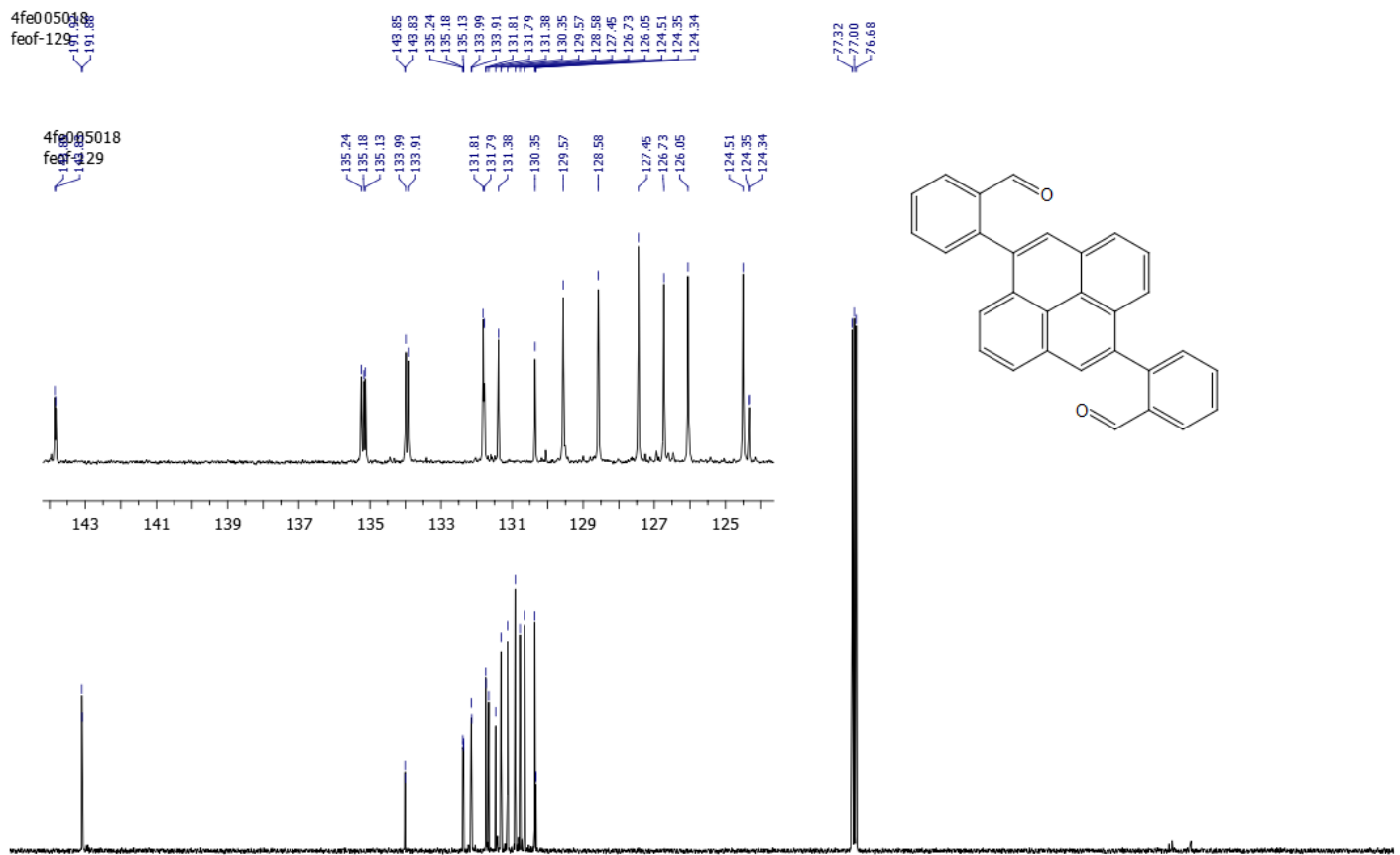

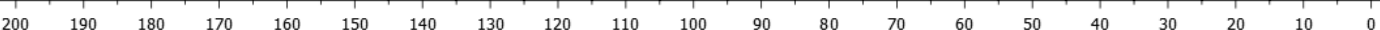

Figure S26. ${ }^{13} \mathrm{C}$ NMR (101 MHz, $\left.\mathrm{CDCl}_{3}, 293 \mathrm{~K}\right)$ spectrum of $\mathbf{P 5}$. 


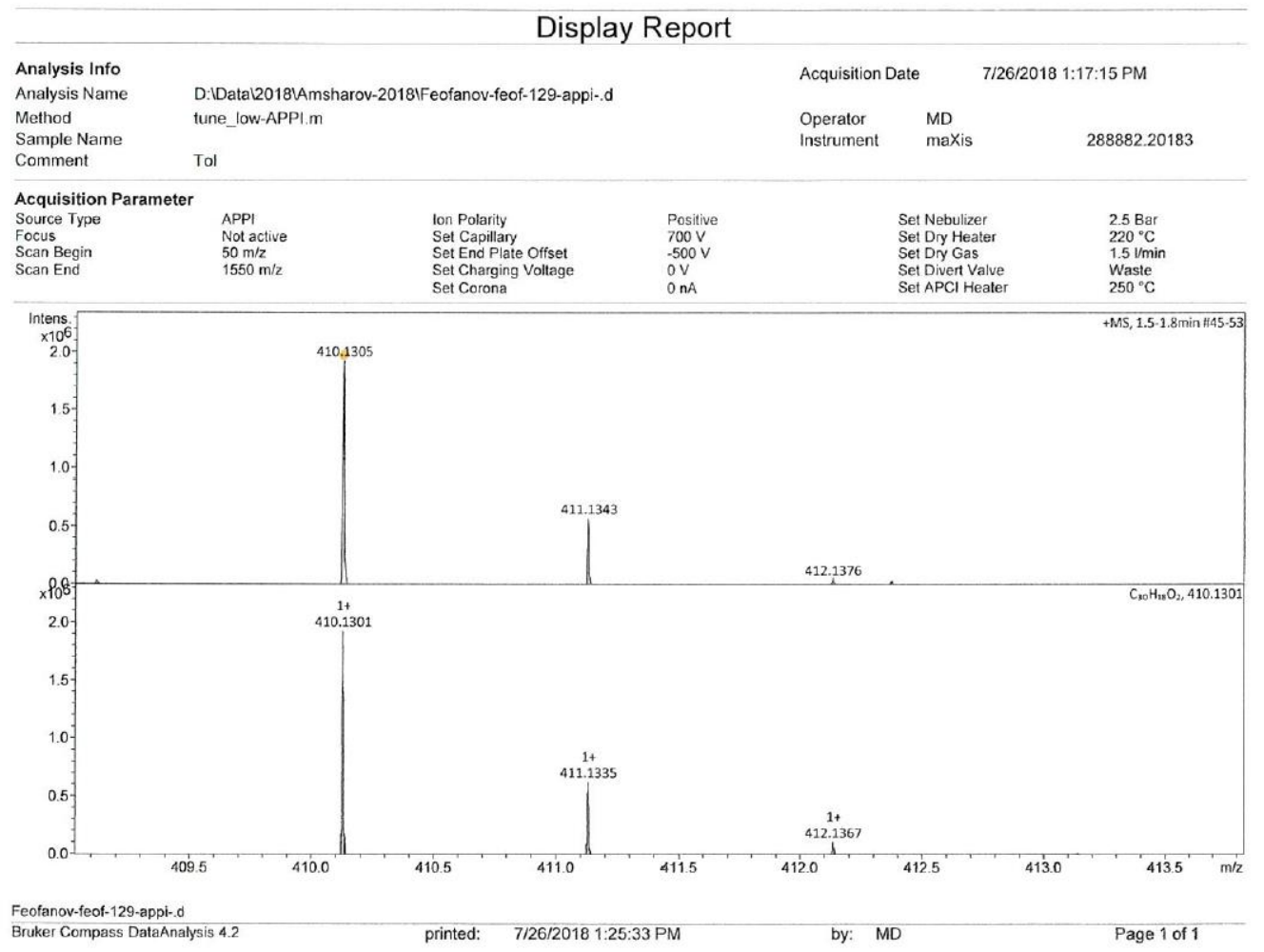

Figure S27. APPI-HRMS spectrum of P5.

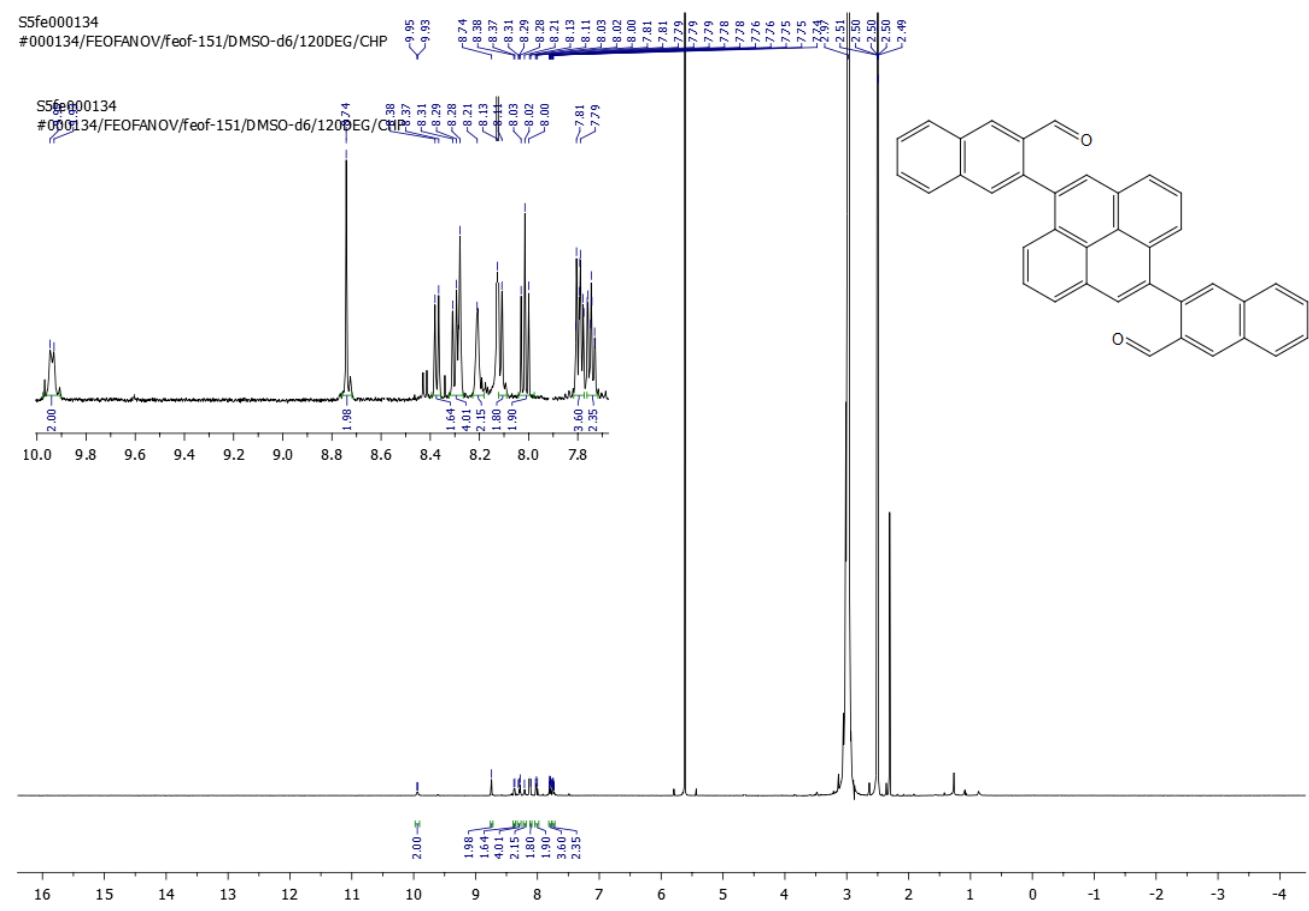

Figure S28. ${ }^{1} \mathrm{H}$ NMR (500 MHz, DMSO-d 6 , 393 K) spectrum of P6. 
Display Report

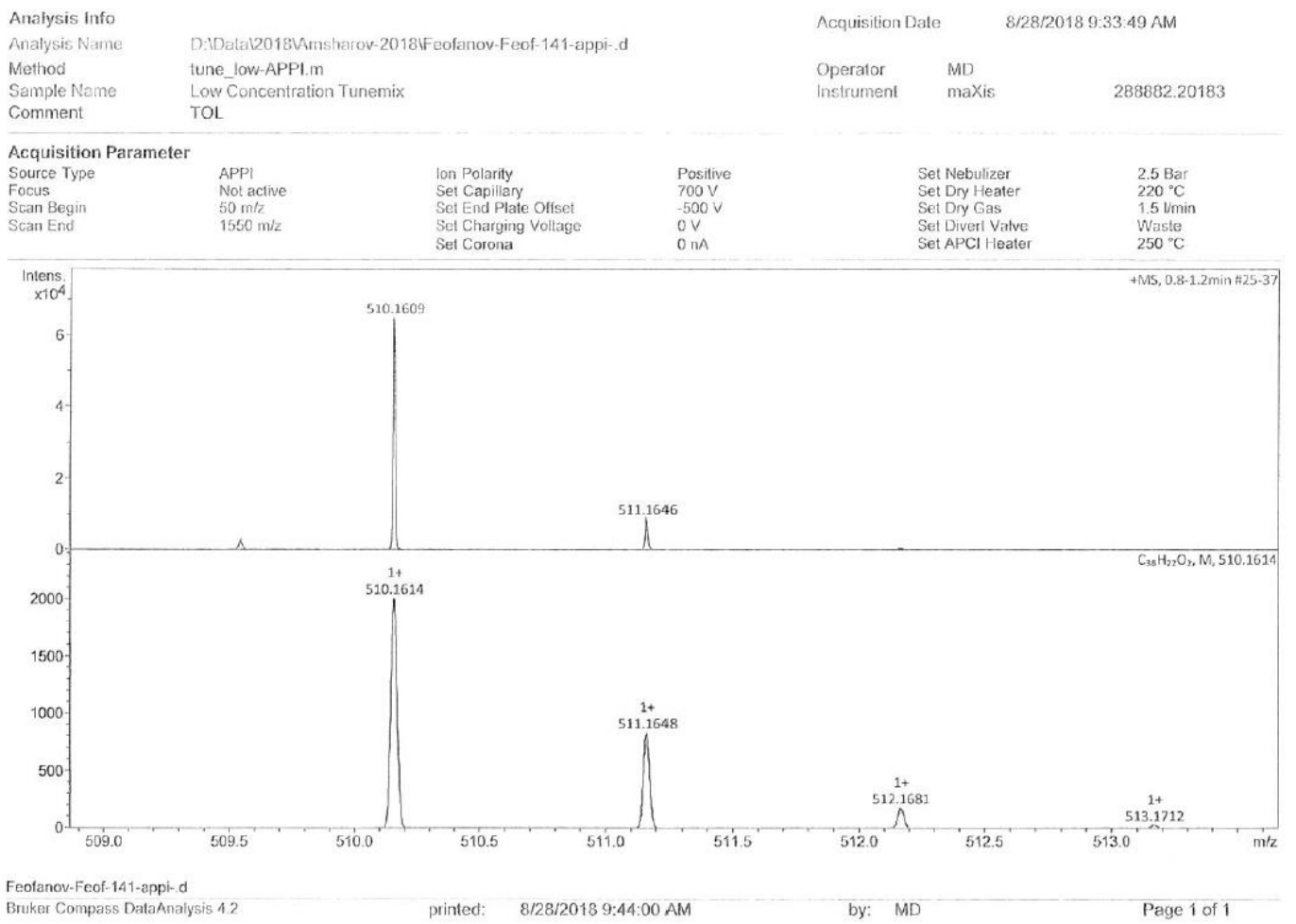

Figure S29. APPI-HRMS spectrum of $\mathbf{P 6}$.

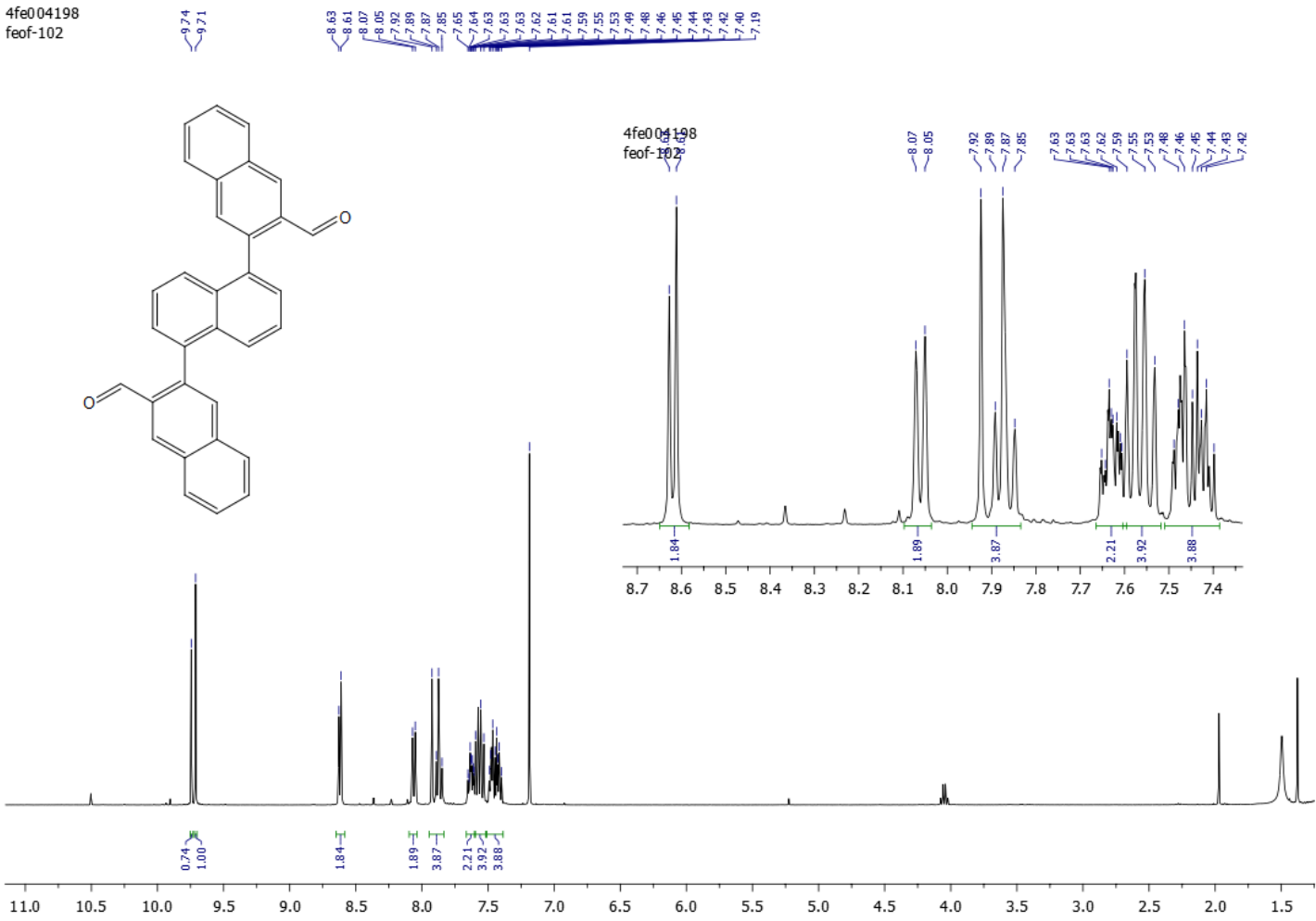

Figure S30. ${ }^{1} \mathrm{H}$ NMR (400 MHz, $\mathrm{CDCl}_{3}, 293 \mathrm{~K}$ ) spectrum of $\mathbf{P 7}$. 


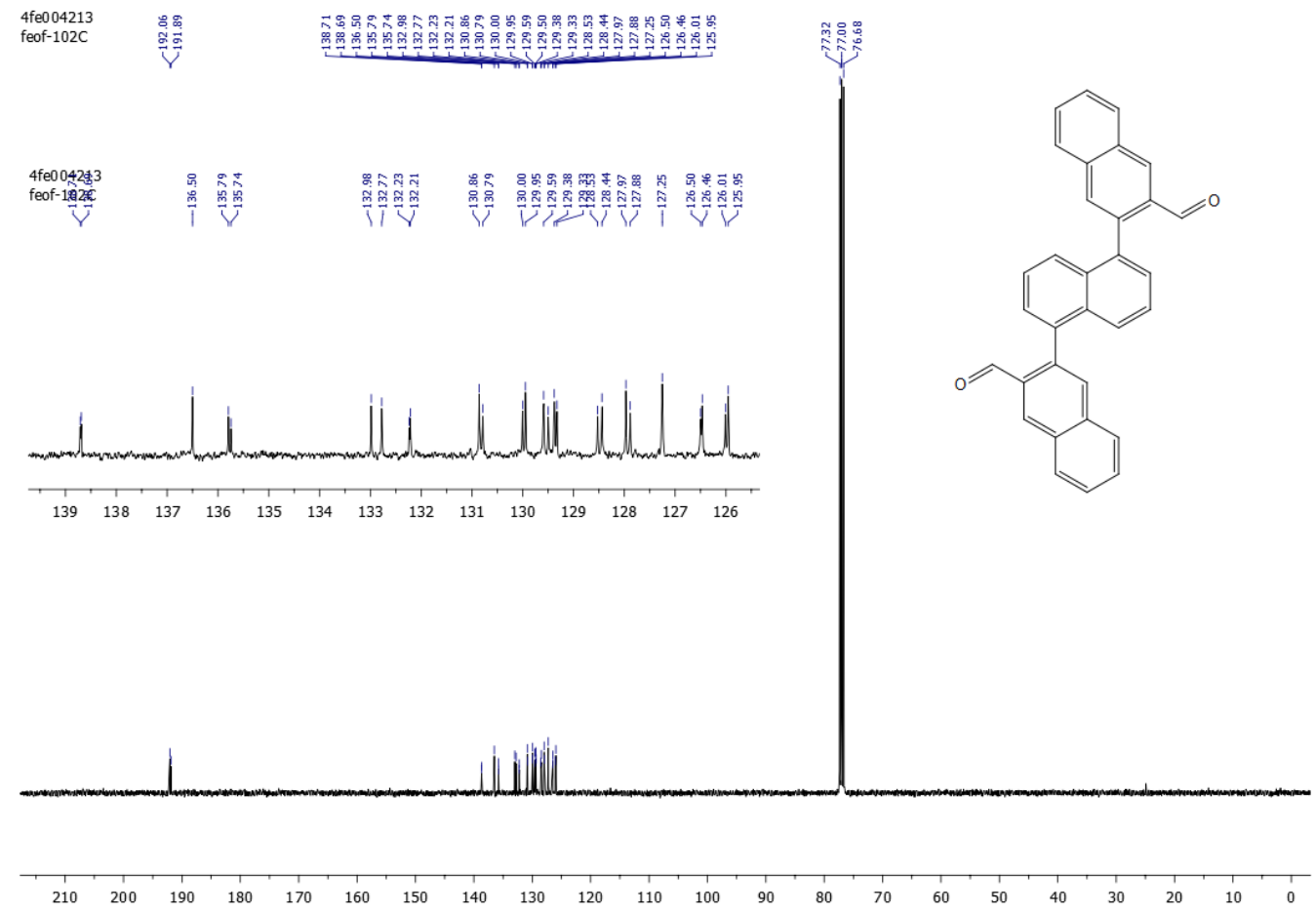

Figure S31. ${ }^{13} \mathrm{C}$ NMR (101 MHz, $\mathrm{CDCl}_{3}, 293 \mathrm{~K}$ ) spectrum of P7.

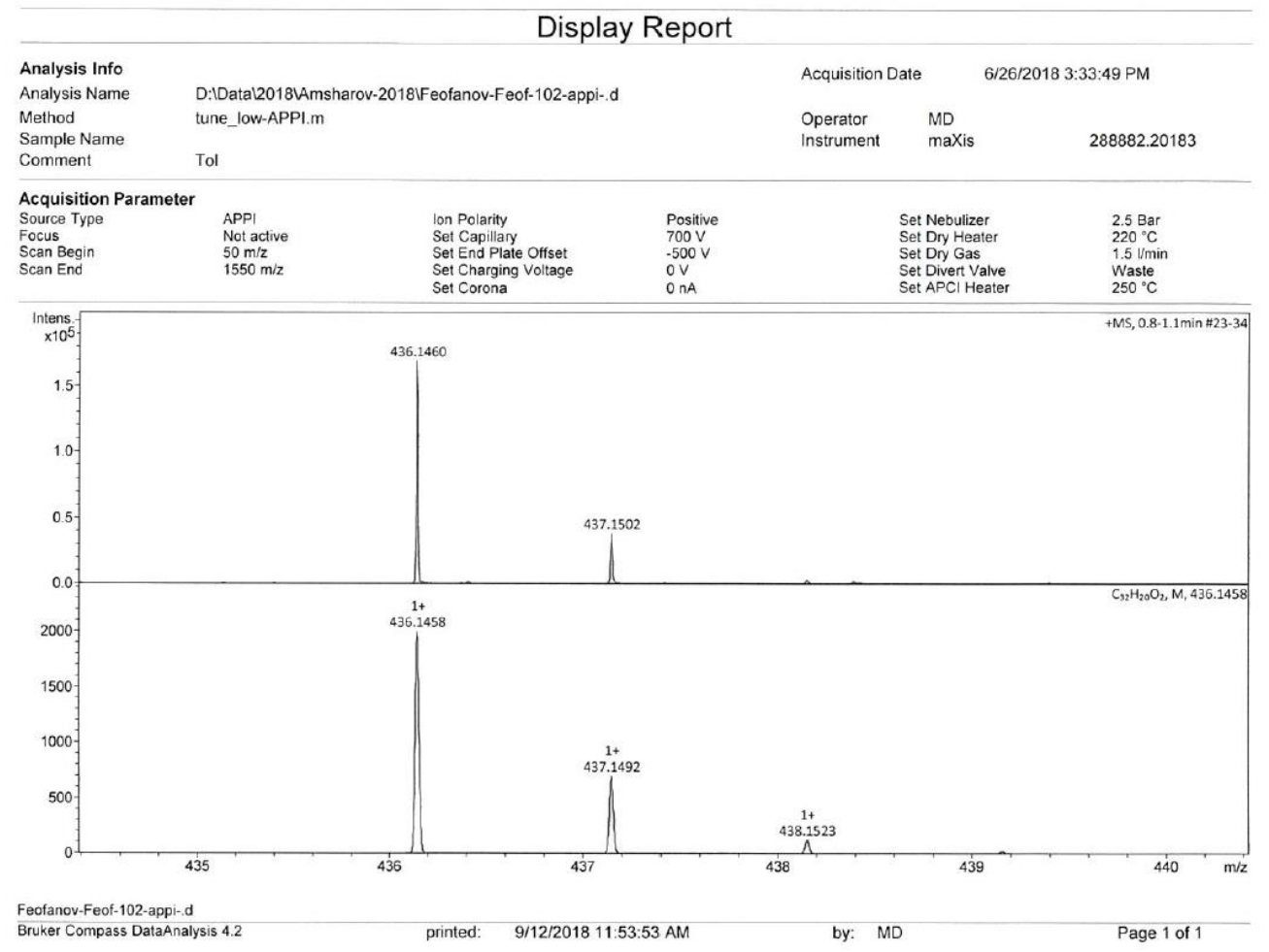

Figure S32. APPI-HRMS spectrum of P7. 


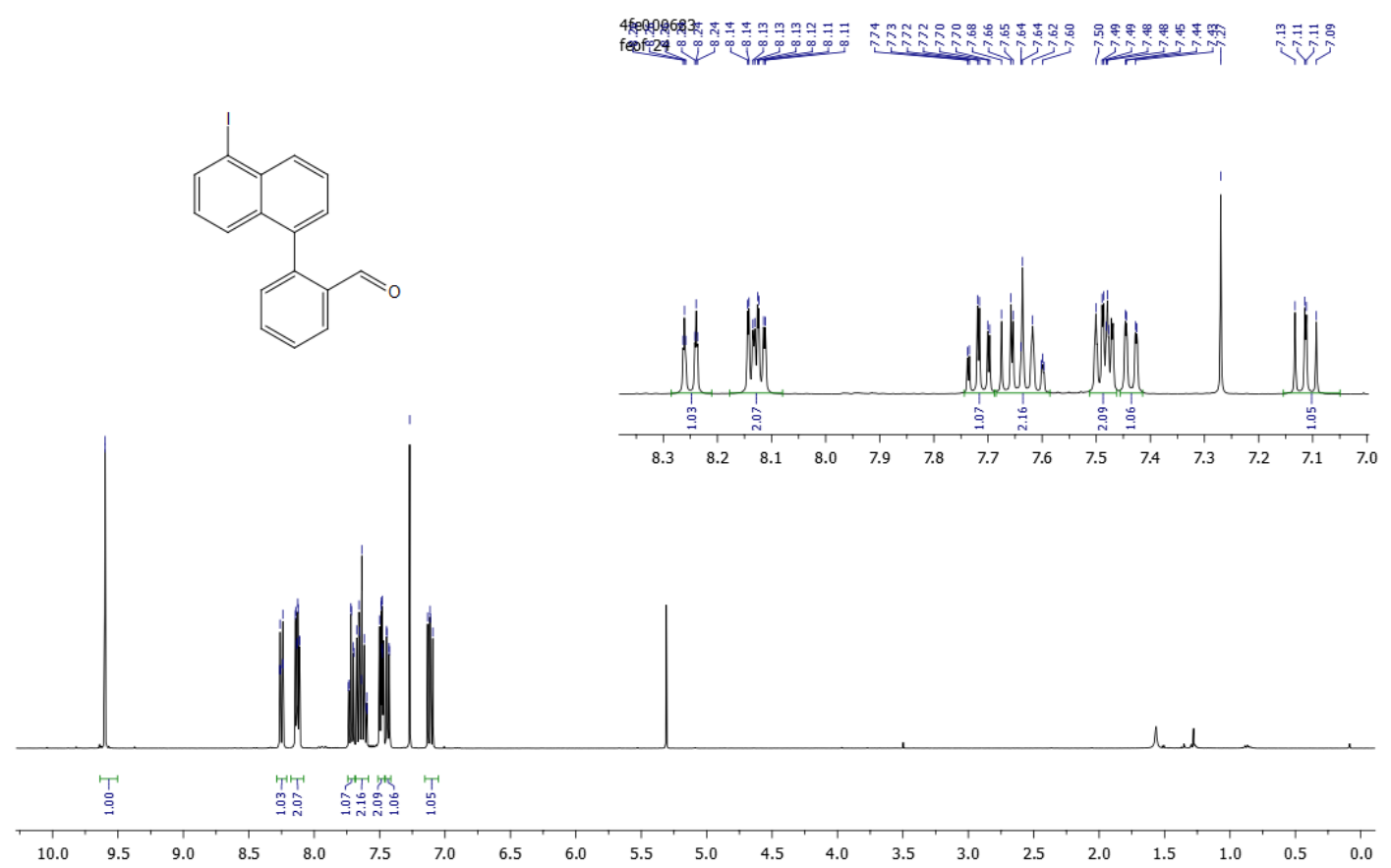

Figure S33. ${ }^{1} \mathrm{H}$ NMR (400 MHz, $\mathrm{CDCl}_{3}, 293 \mathrm{~K}$ ) spectrum of 2-(5-iodonaphthalen-1yl)benzaldehyde.

$4 \mathrm{e} 000785$

$$
\text { 产 }
$$
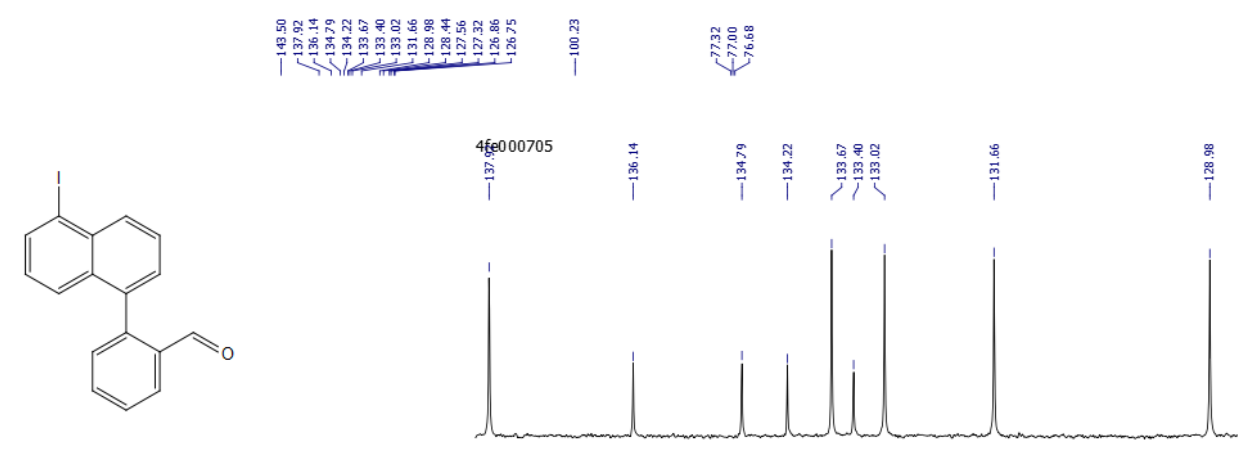

$$
\stackrel{\top}{138}
$$
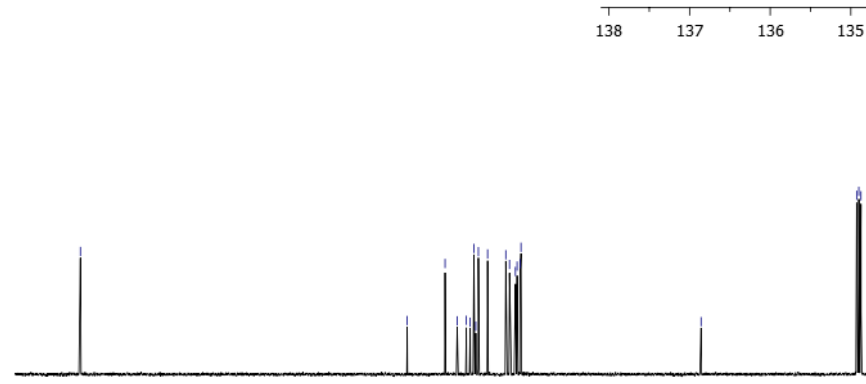$$
200
$$$$
200
$$

Figure S34. ${ }^{13} \mathrm{C}$ NMR $\left(101 \mathrm{MHz}, \mathrm{CDCl}_{3}, 293 \mathrm{~K}\right)$ spectrum of 2-(5-iodonaphthalen-1yl)benzaldehyde. 


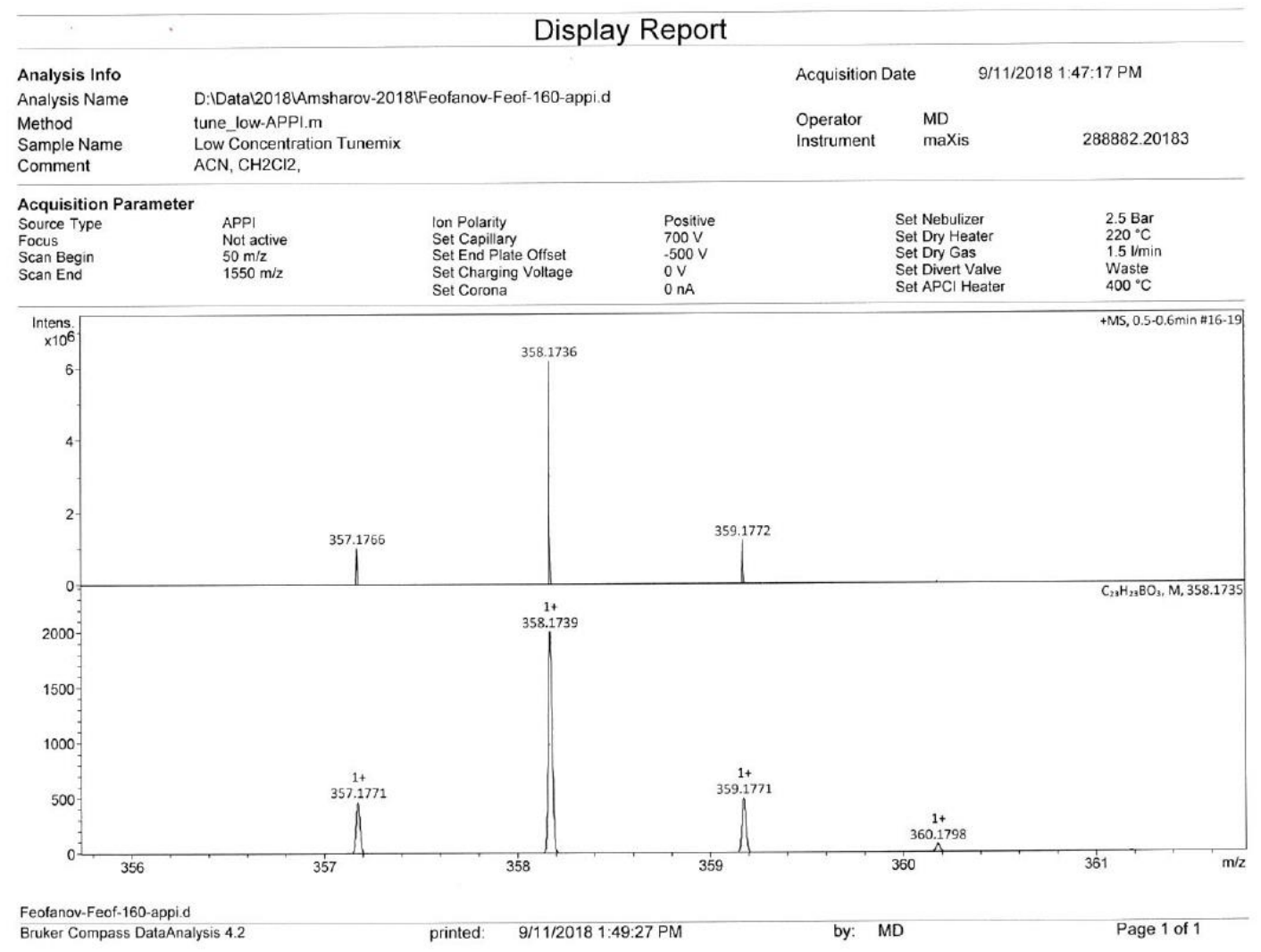

Figure S35. APPI-HRMS spectrum of 2-(5-iodonaphthalen-1-yl)benzaldehyde.

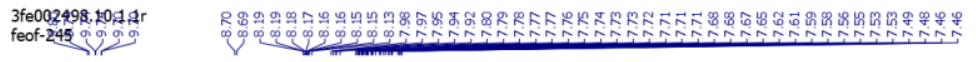

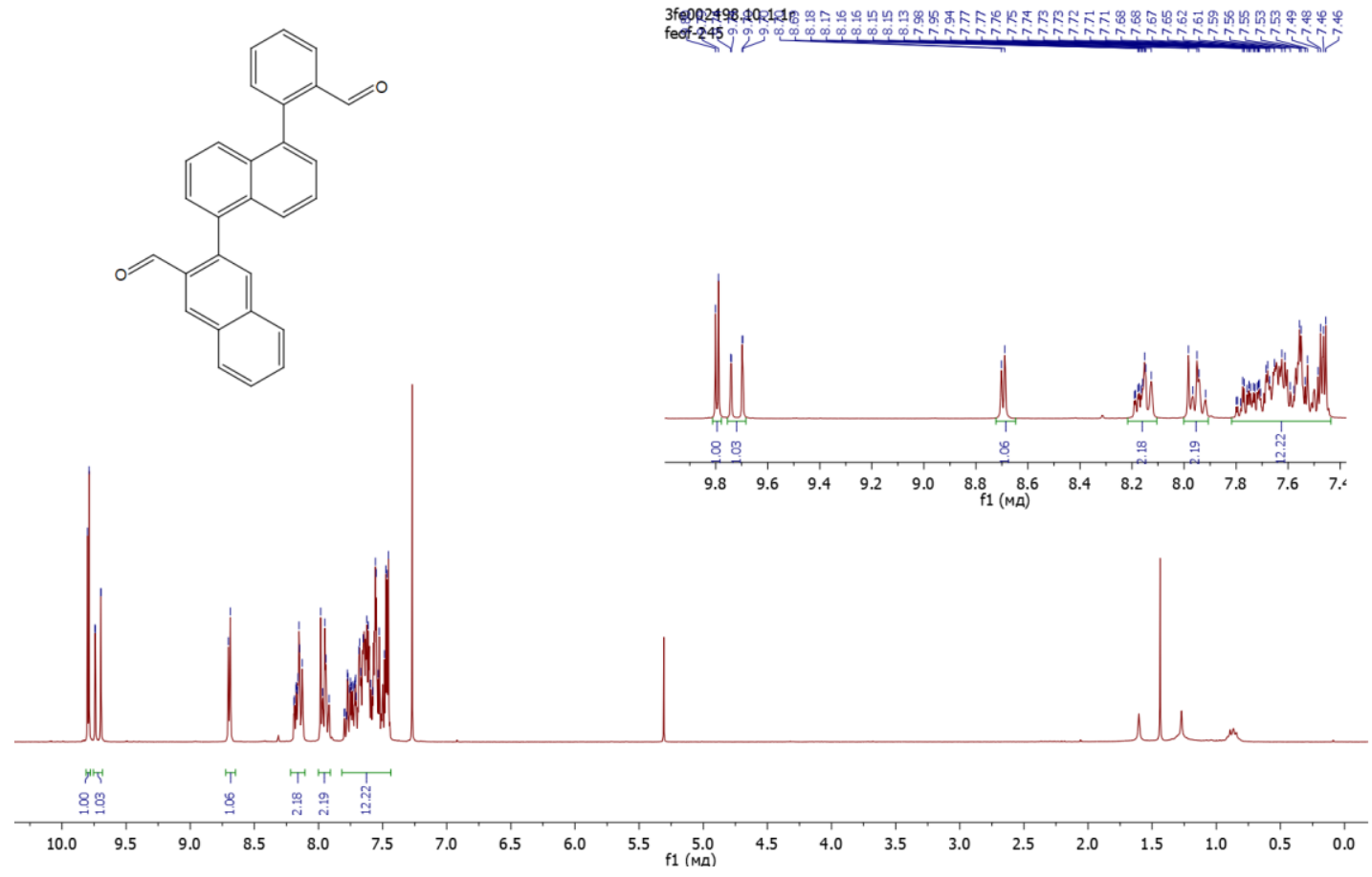

Figure S36. ${ }^{1} \mathrm{H}$ NMR $\left(300 \mathrm{MHz}, \mathrm{CDCl}_{3}, 293 \mathrm{~K}\right)$ spectrum of $\mathbf{P 8}$. 

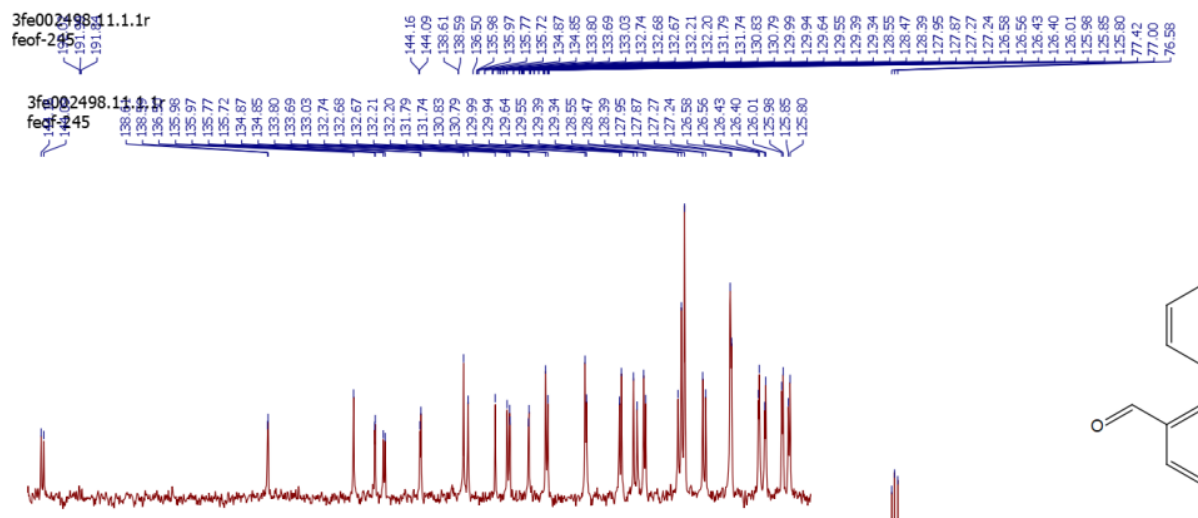

$\begin{array}{lllllllllllllllllllllll}144 & 143 & 142 & 141 & 140 & 139 & 138 & 137 & 136 & 135 & 134 & 133 & 132 & 131 & 130 & 129 & 128 & 127 & 126\end{array}$
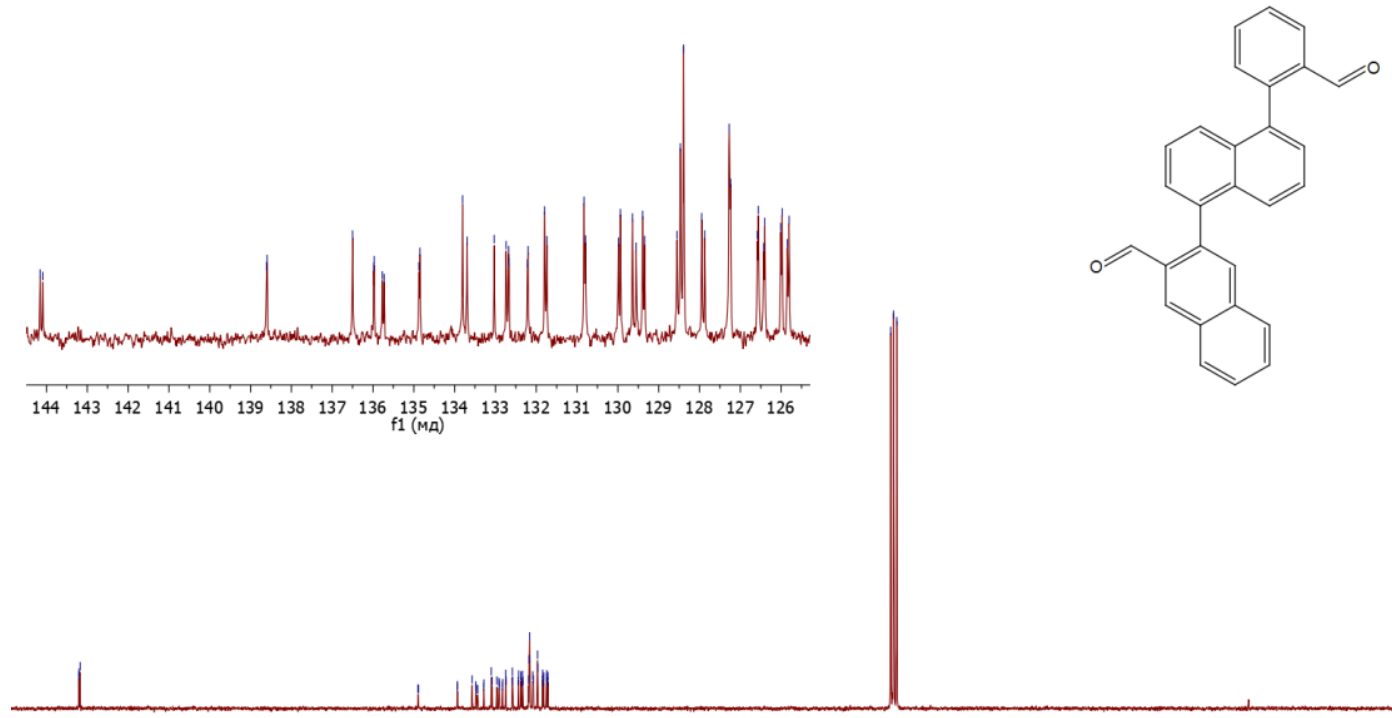

\begin{tabular}{lllllllllllllllllllllll}
\hline & 190 & 190 & 180 & 170 & 160 & 150 & 140 & 130 & 120 & 110 & 100 & 90 & 80 & 70 & 60 & 50 & 40 & 30 & 20 & 10 & 0
\end{tabular}

Figure S37. ${ }^{13} \mathrm{C}$ NMR (78 MHz, $\left.\mathrm{CDCl}_{3}, 293 \mathrm{~K}\right)$ spectrum of $\mathbf{P 8}$.

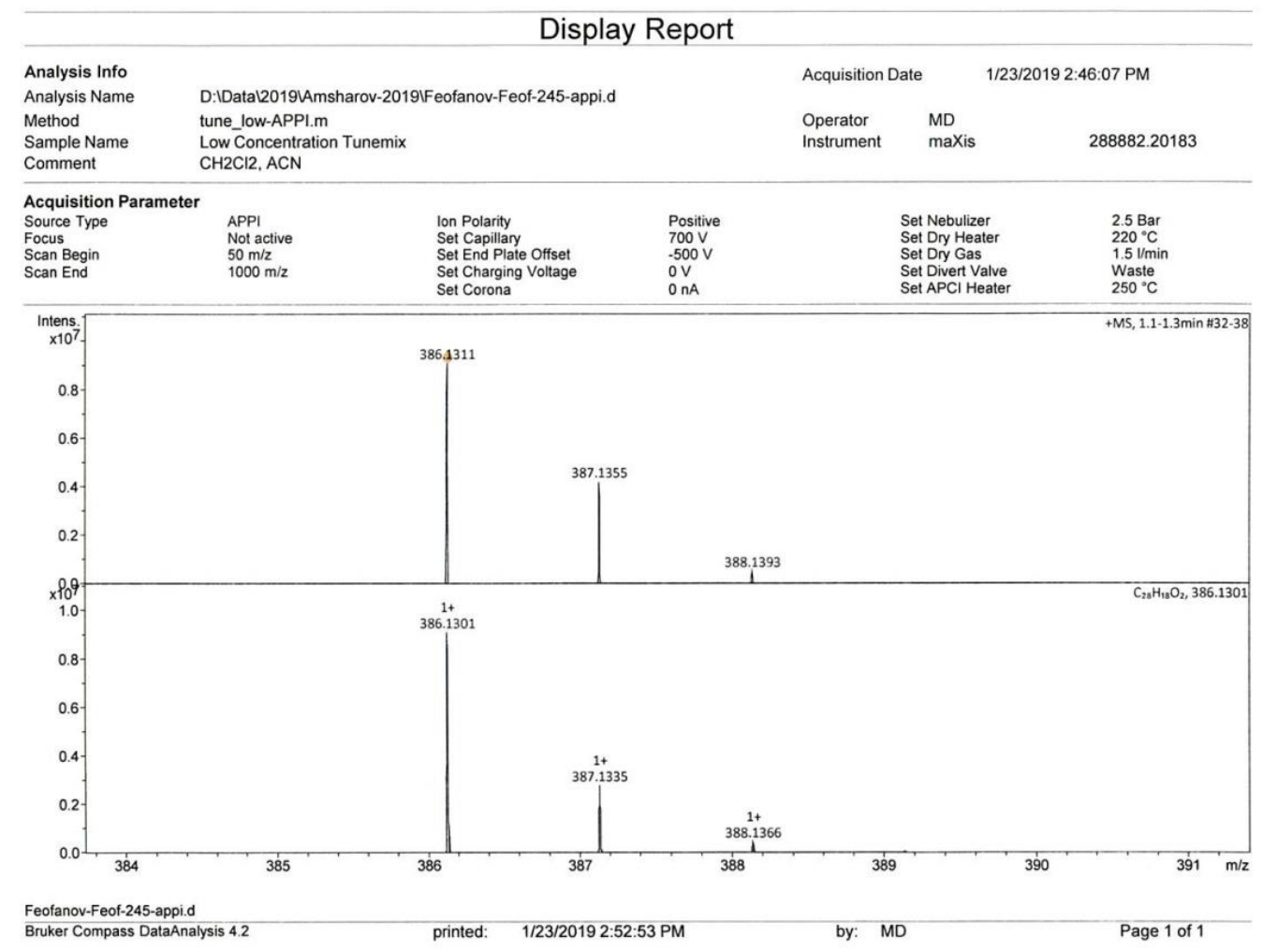

Figure S38. APPI-HRMS spectrum of $\mathbf{P 8}$. 

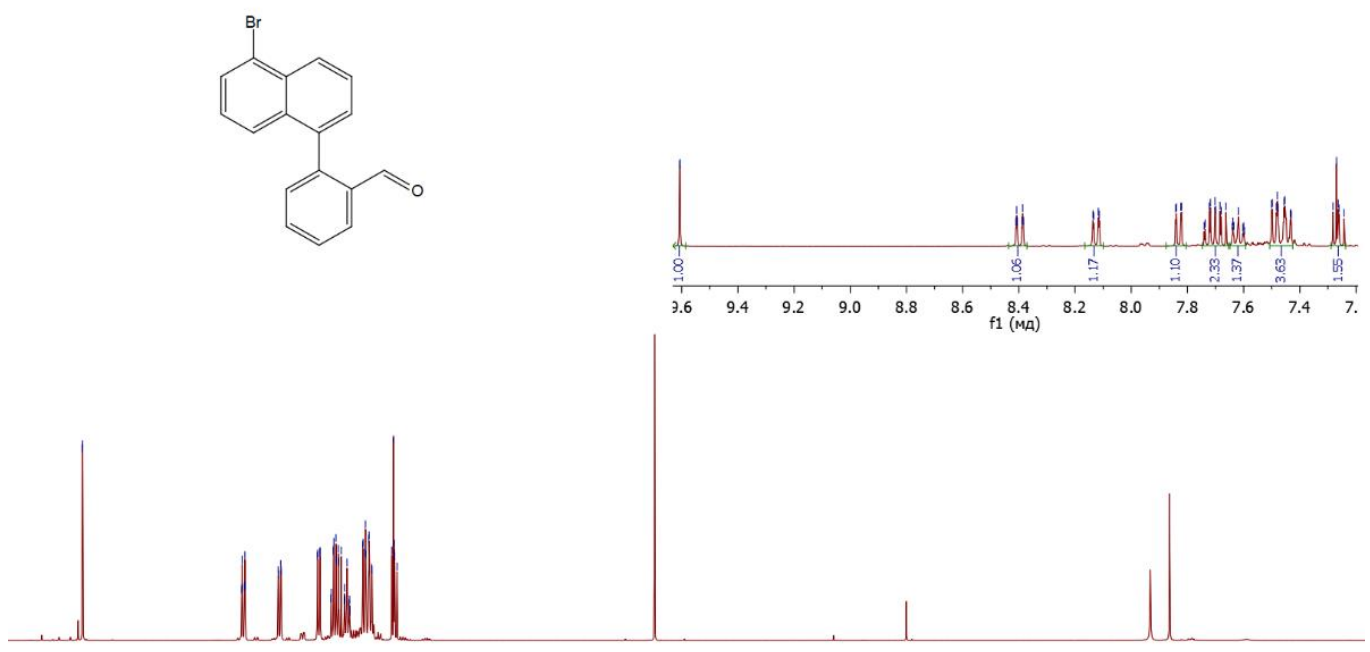

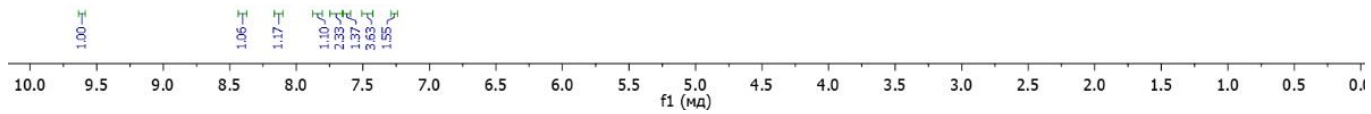

Figure S39. ${ }^{1} \mathrm{H} \mathrm{NMR}\left(400 \mathrm{MHz}, \mathrm{CDCl}_{3}, 293 \mathrm{~K}\right)$ spectrum of 2-(5-bromonaphthalen-1yl)benzaldehyde.

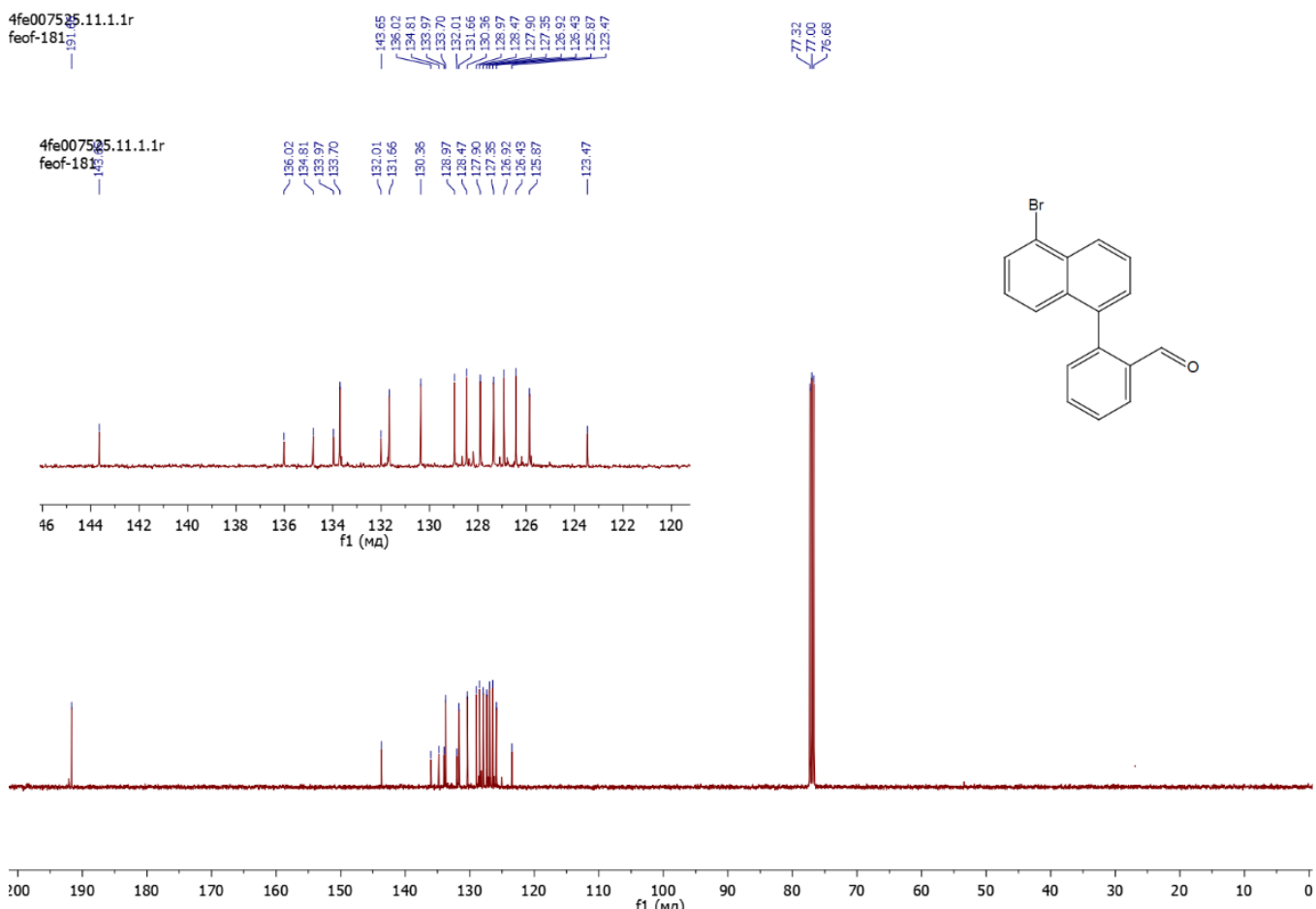

Figure S40. ${ }^{13} \mathrm{C}$ NMR $\left(101 \mathrm{MHz}, \mathrm{CDCl}_{3}, 293 \mathrm{~K}\right)$ spectrum of 2-(5-bromonaphthalen-1yl)benzaldehyde. 


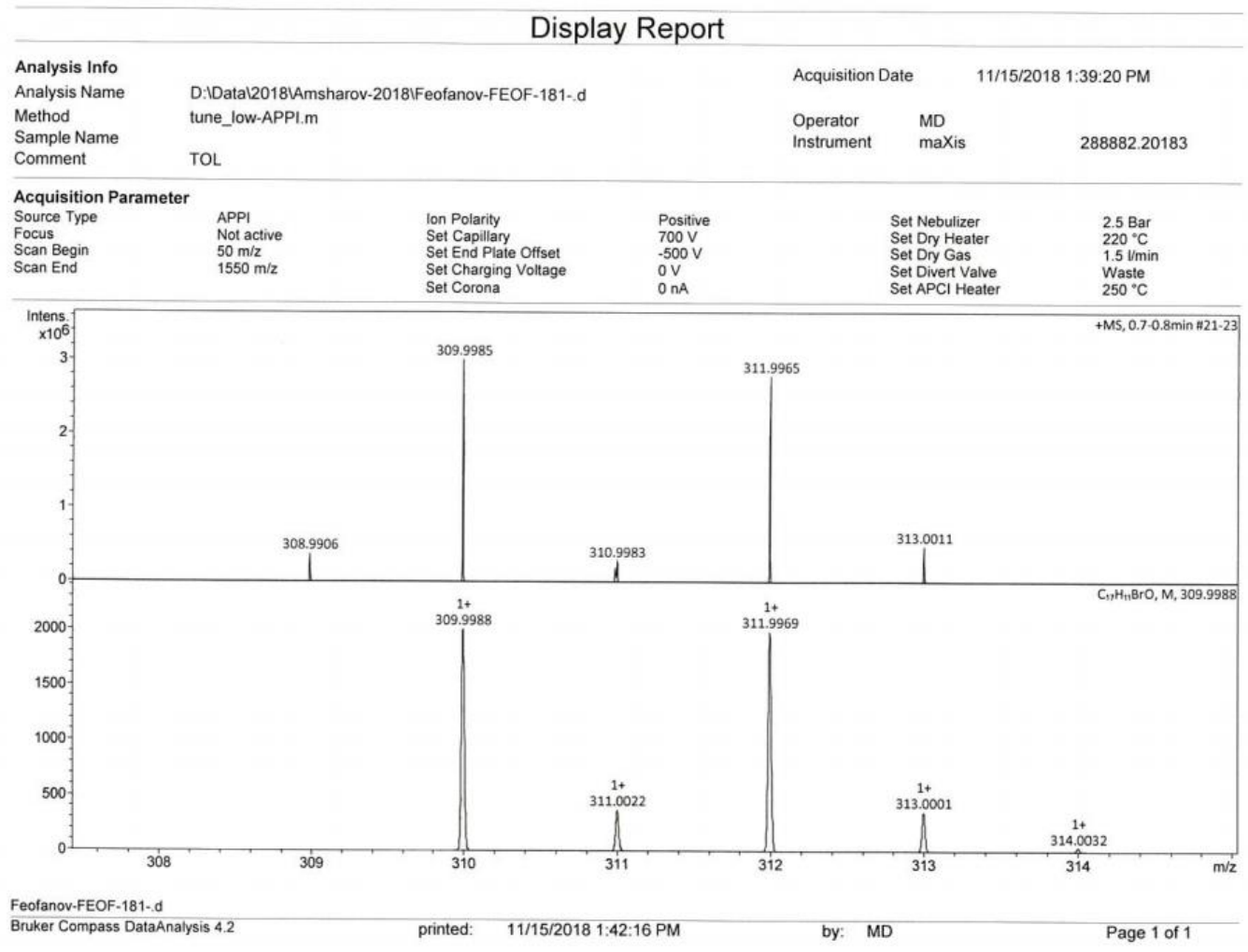

Figure S41. APPI-HRMS spectrum of 2-(5-bromonaphthalen-1-yl)benzaldehyde.

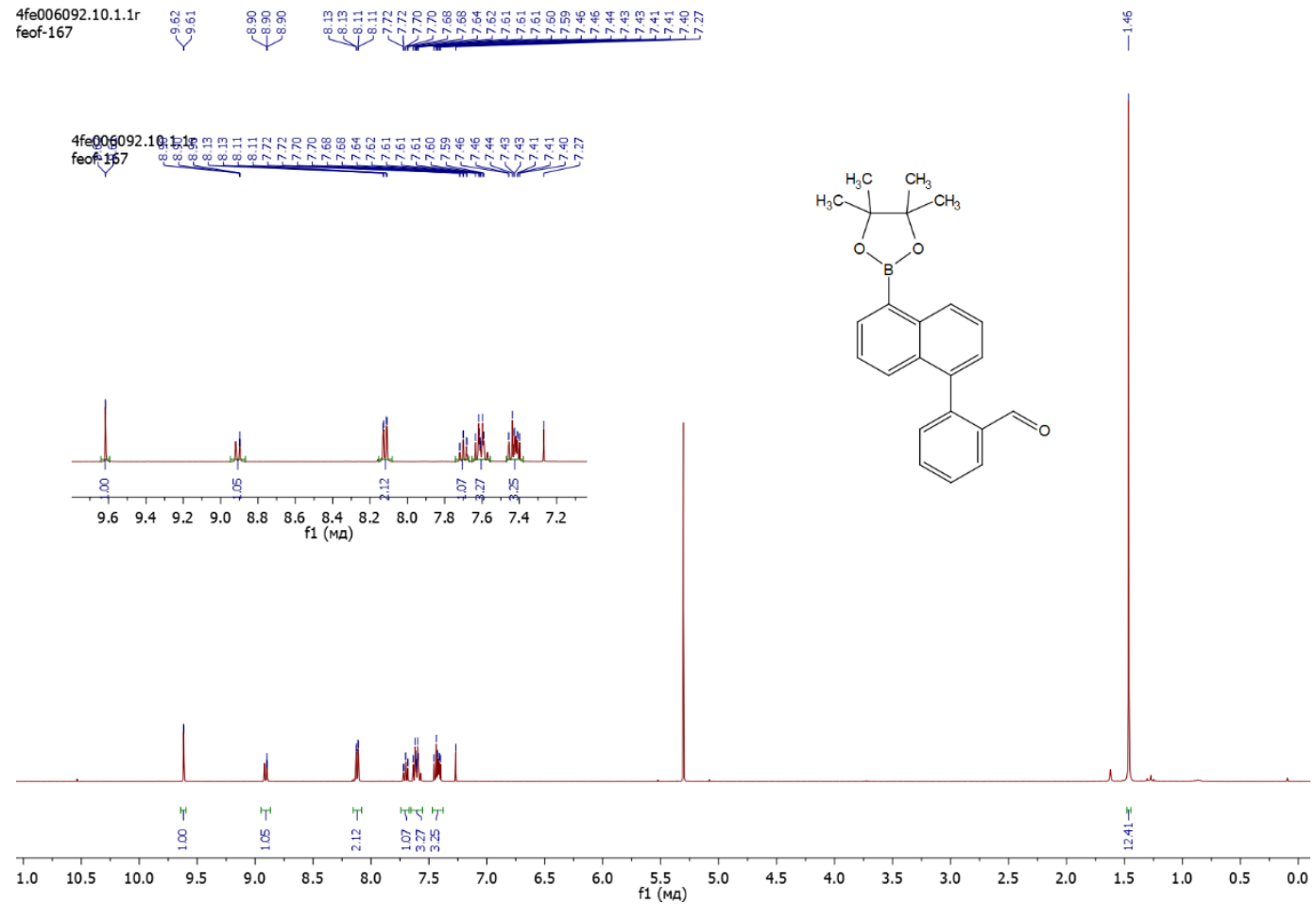

Figure S42. ${ }^{1} \mathrm{H}$ NMR (400 MHz, $\left.\mathrm{CDCl}_{3}, 293 \mathrm{~K}\right)$ spectrum of 2-(5-(4,4,5,5-tetramethyl-1,3,2dioxaborolan-2-yl)naphthalen-1-yl)benzaldehyde. 


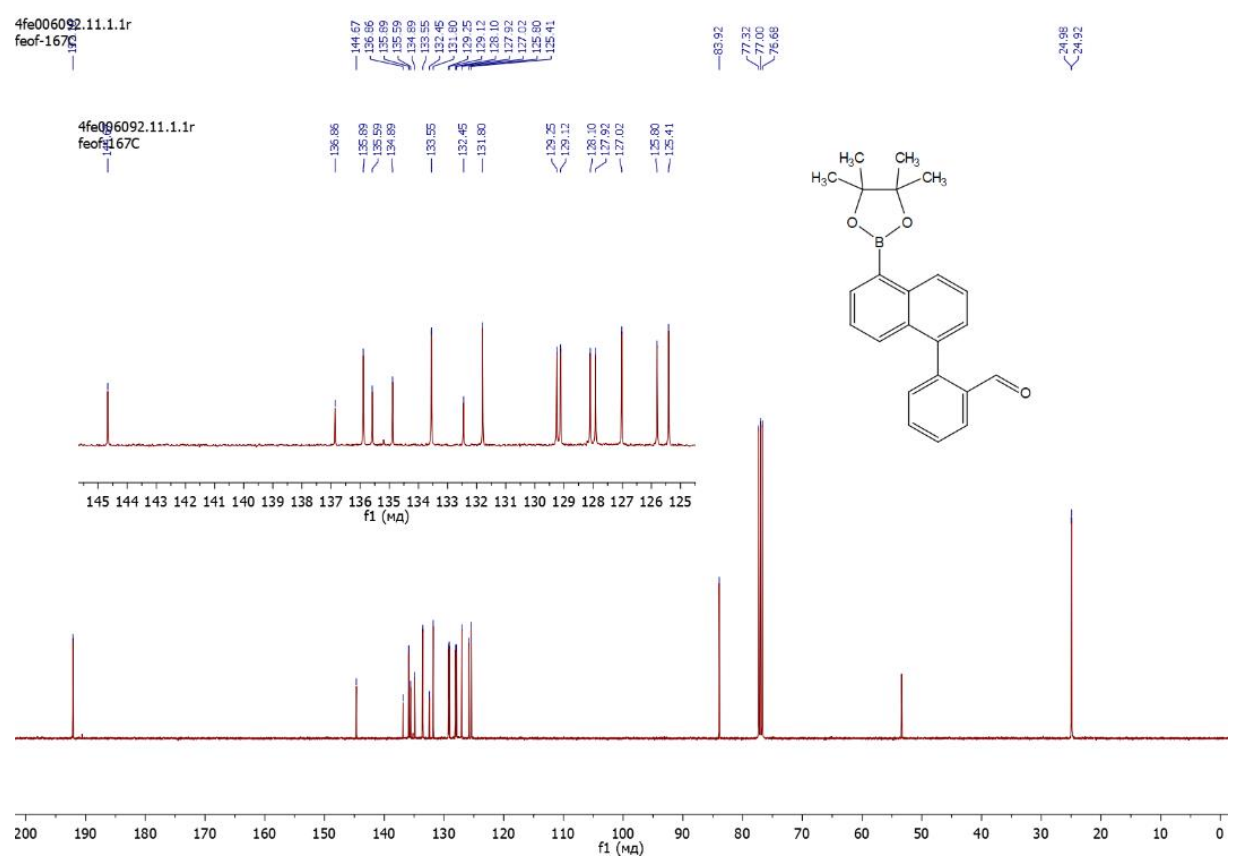

Figure S43. ${ }^{13} \mathrm{C}$ NMR $\left(101 \mathrm{MHz}, \mathrm{CDCl}_{3}, 293 \mathrm{~K}\right)$ spectrum of 2-(5-(4,4,5,5-tetramethyl-1,3,2dioxaborolan-2-yl)naphthalen-1-yl)benzaldehyde.

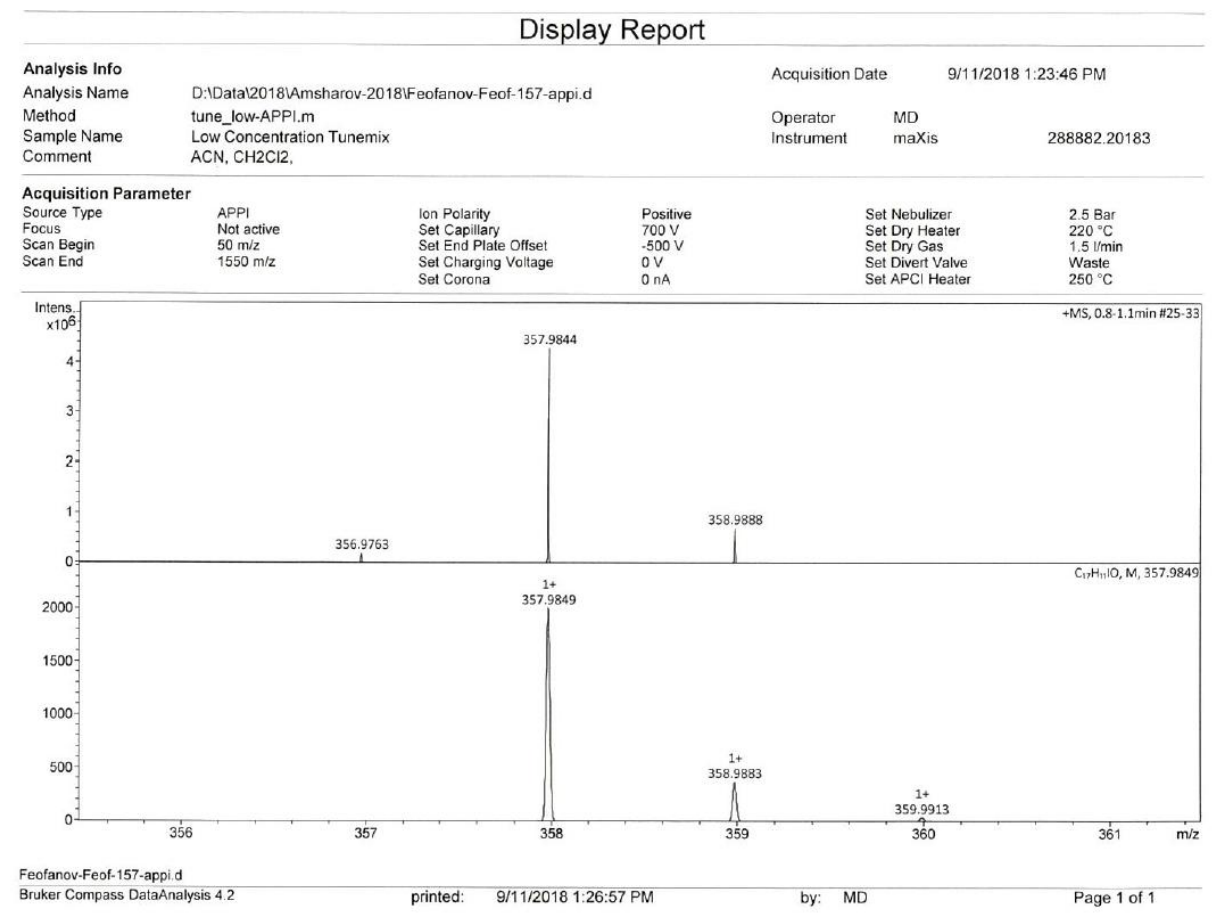

Figure S44. APPI-HRMS spectrum of 2-(5-(4,4,5,5-tetramethyl-1,3,2-dioxaborolan-2yl)naphthalen-1-yl)benzaldehyde. 


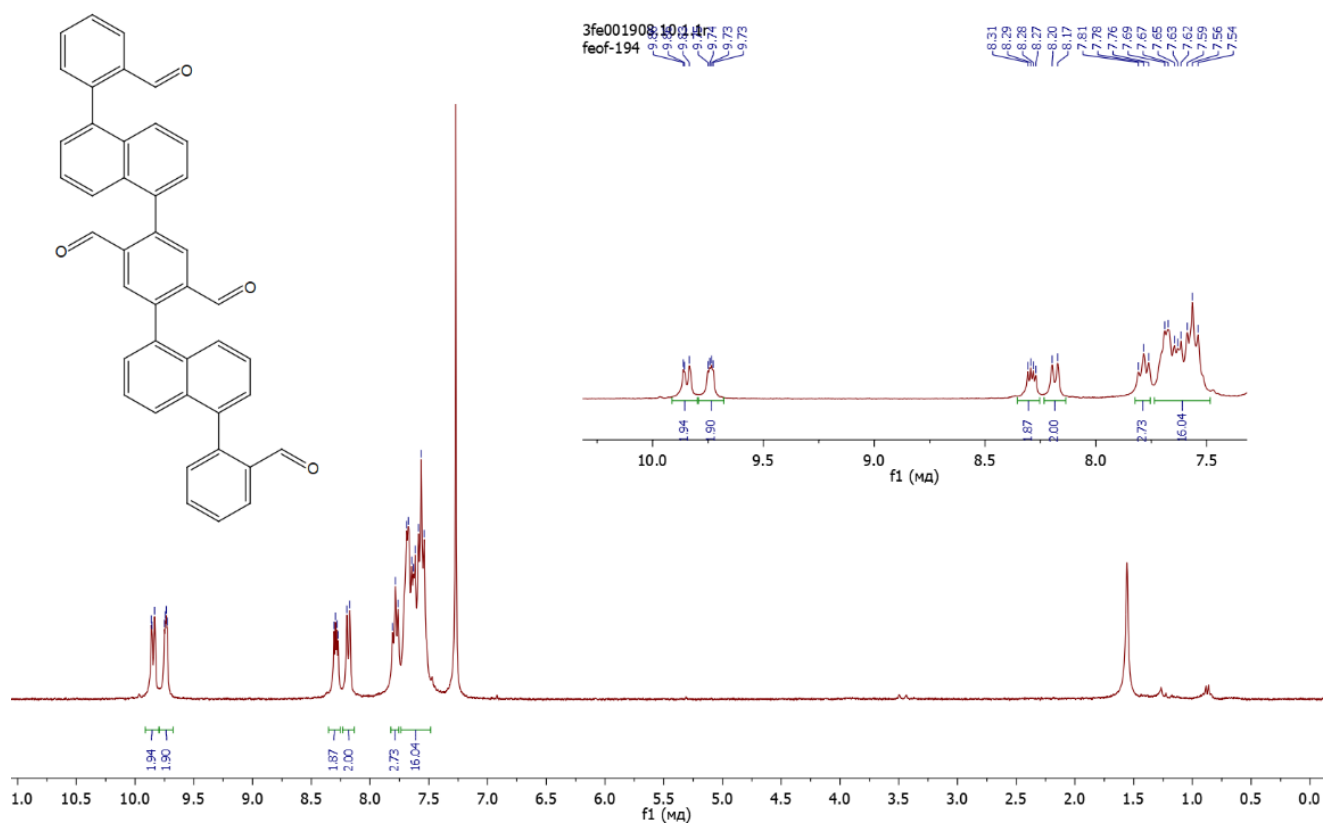

Figure S45. ${ }^{1} \mathrm{H} \mathrm{NMR}\left(400 \mathrm{MHz}, \mathrm{CDCl}_{3}, 293 \mathrm{~K}\right)$ spectrum of $\mathbf{P 9}$.

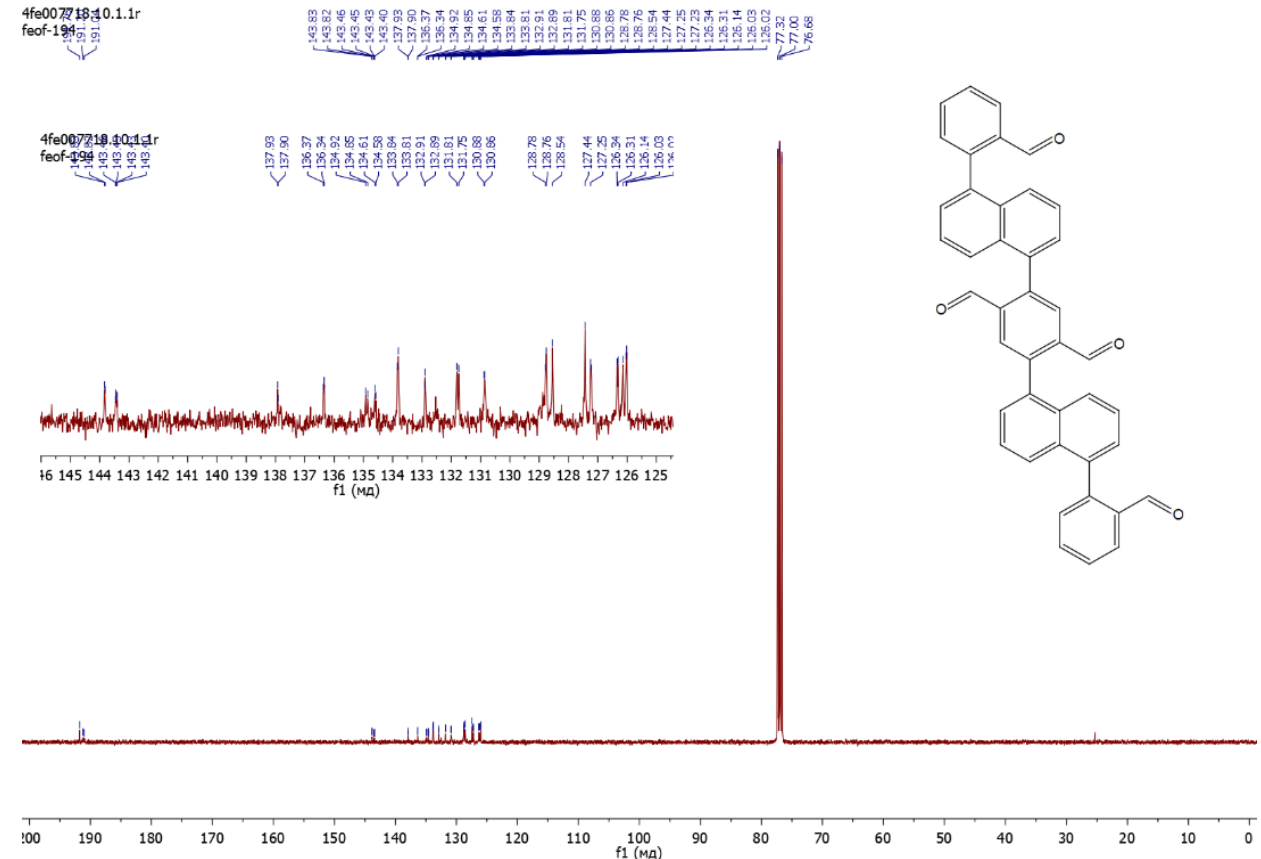

Figure S46. ${ }^{13} \mathrm{C}$ NMR (101 MHz, $\left.\mathrm{CDCl}_{3}, 293 \mathrm{~K}\right)$ spectrum of $\mathbf{P 9}$. 


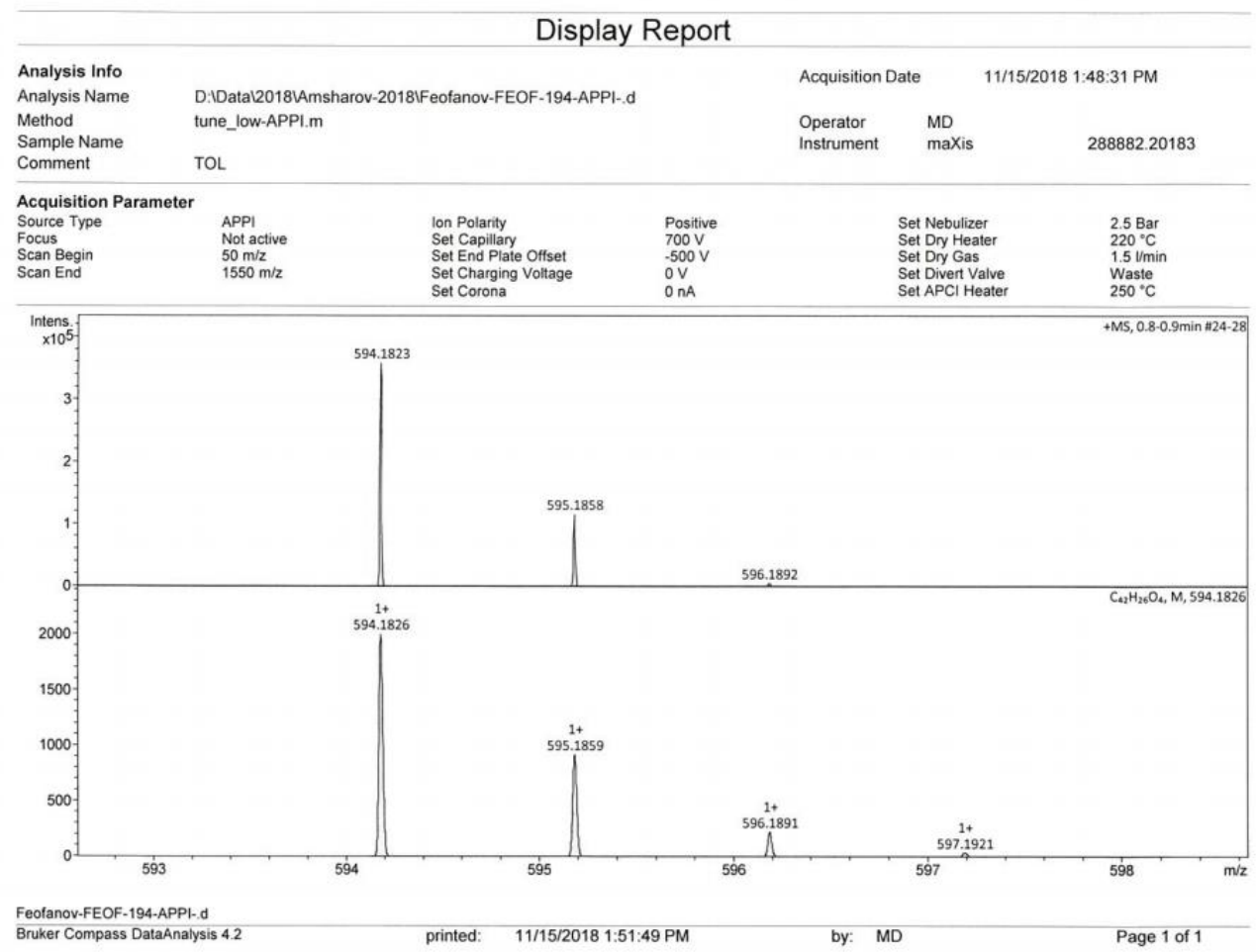

Figure S47. APPI-HRMS spectrum of P9.

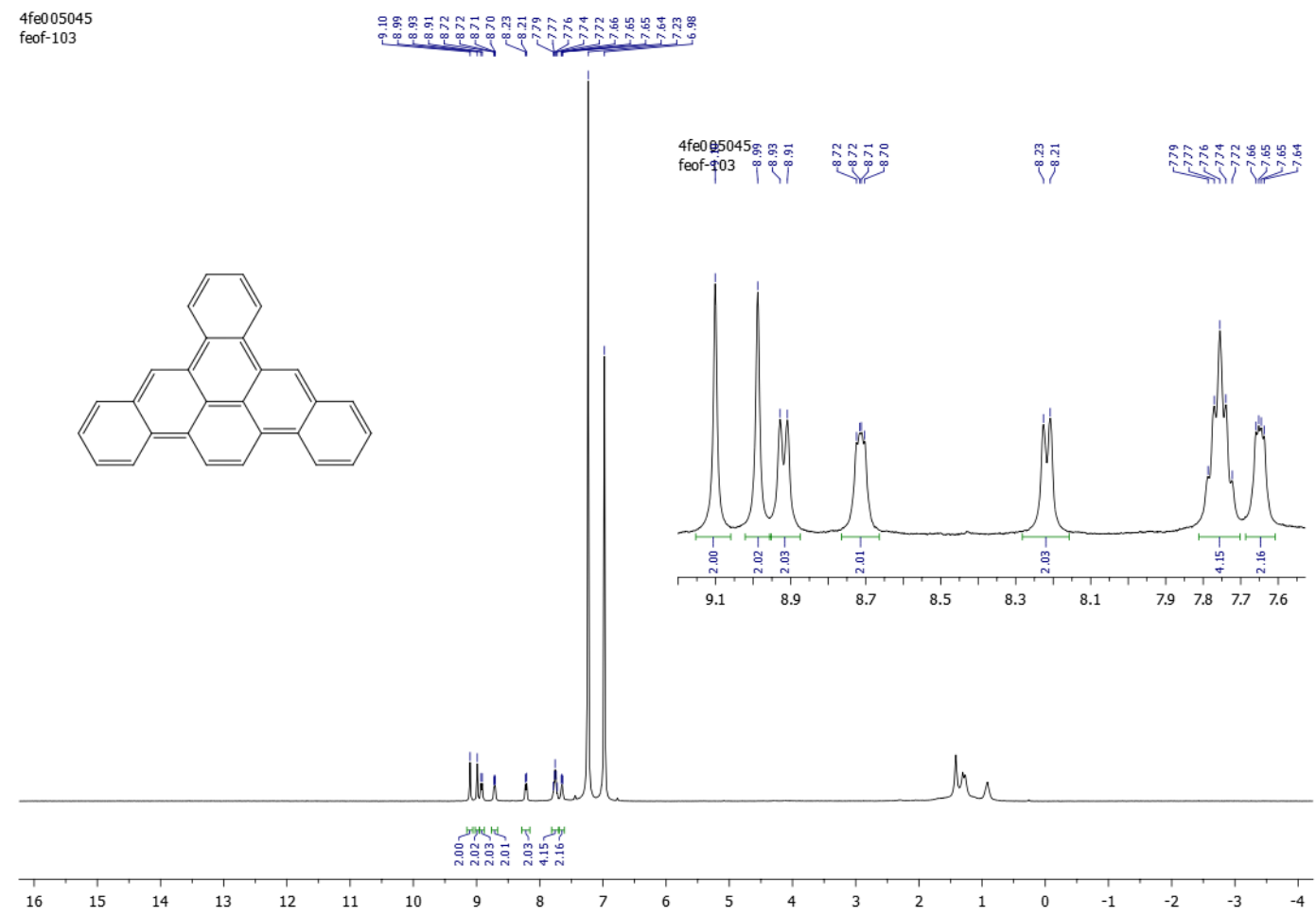

Figure S48. ${ }^{1} \mathrm{H}$ NMR (400 MHz, $\left.o-\mathrm{C}_{6} \mathrm{D}_{4} \mathrm{Cl}_{2}, 293 \mathrm{~K}\right)$ spectrum of NG1. 

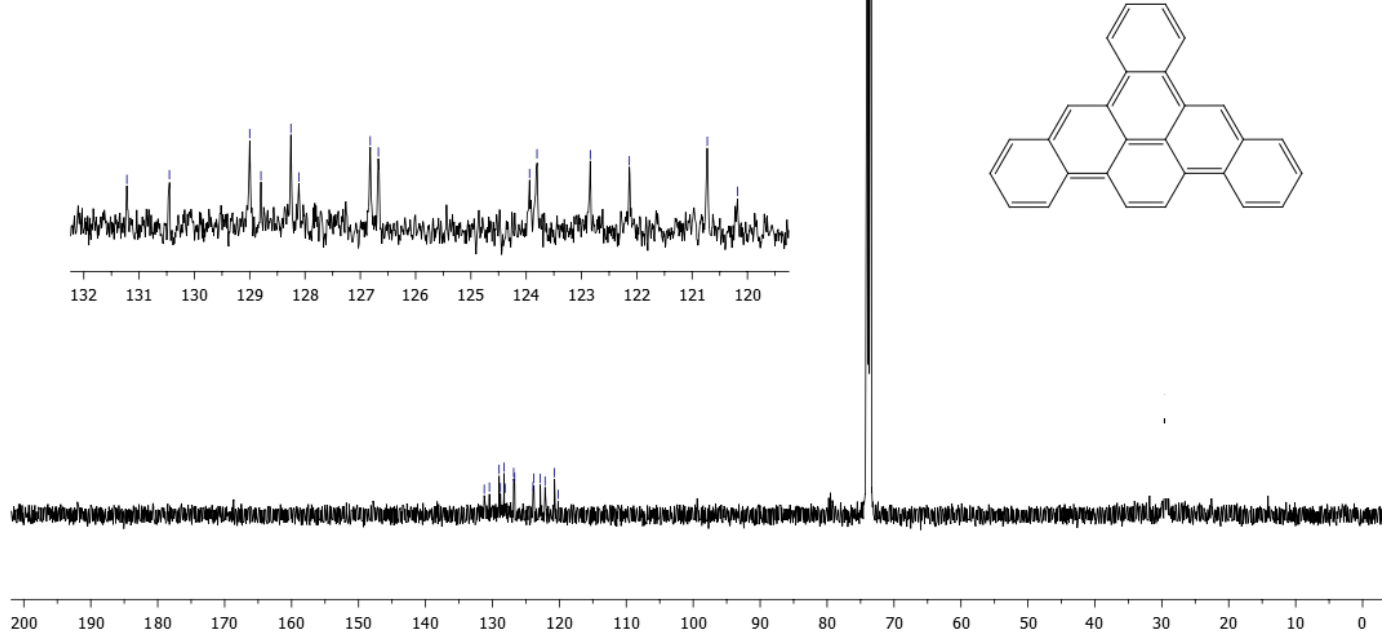

Figure S49. ${ }^{13} \mathrm{C}$ NMR (101 MHz, $\left.\mathrm{C}_{2} \mathrm{D}_{2} \mathrm{Cl}_{4}, 293 \mathrm{~K}\right)$ spectrum of NG1.

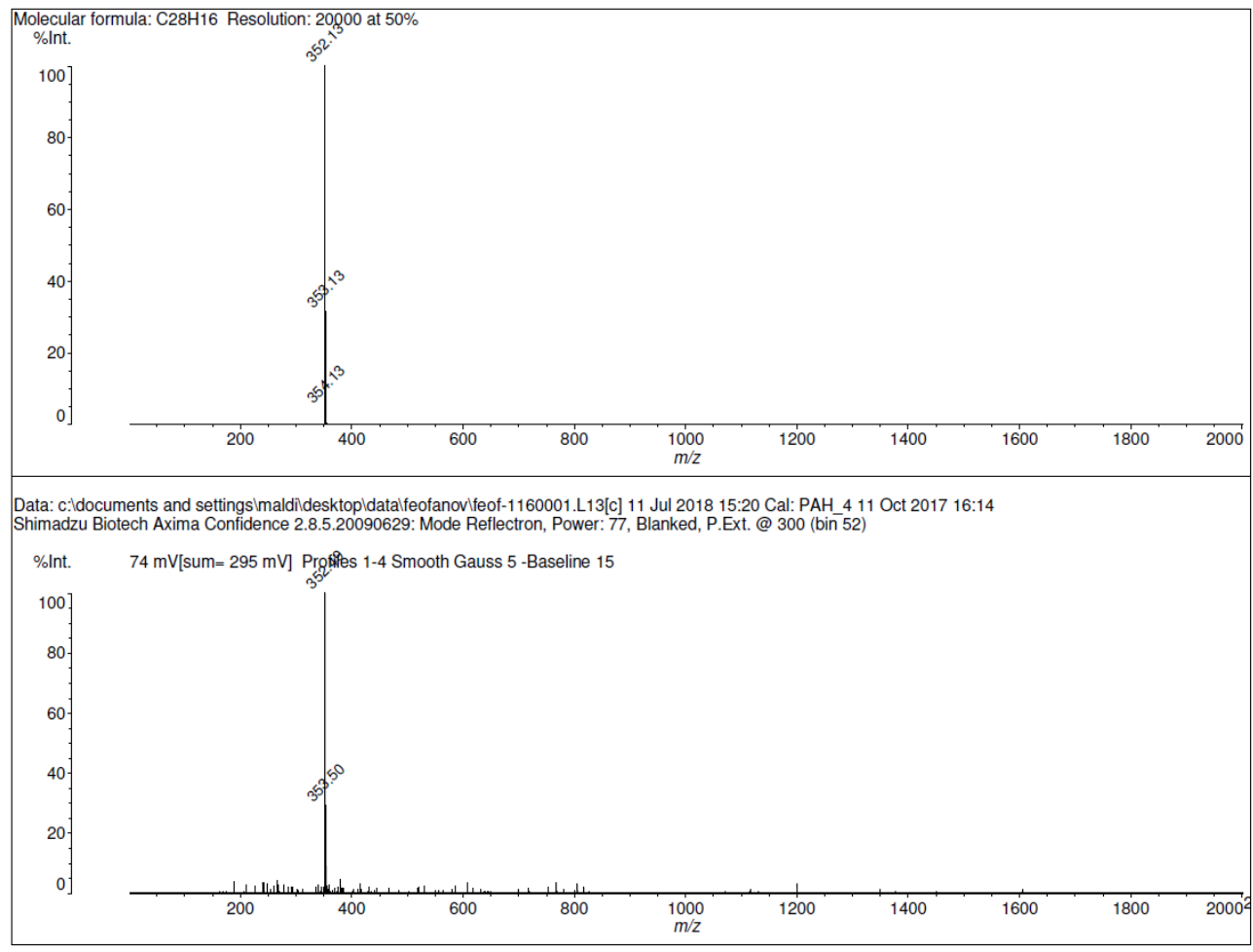

Figure S50. LDI-TOF MS spectrum of NG1. 


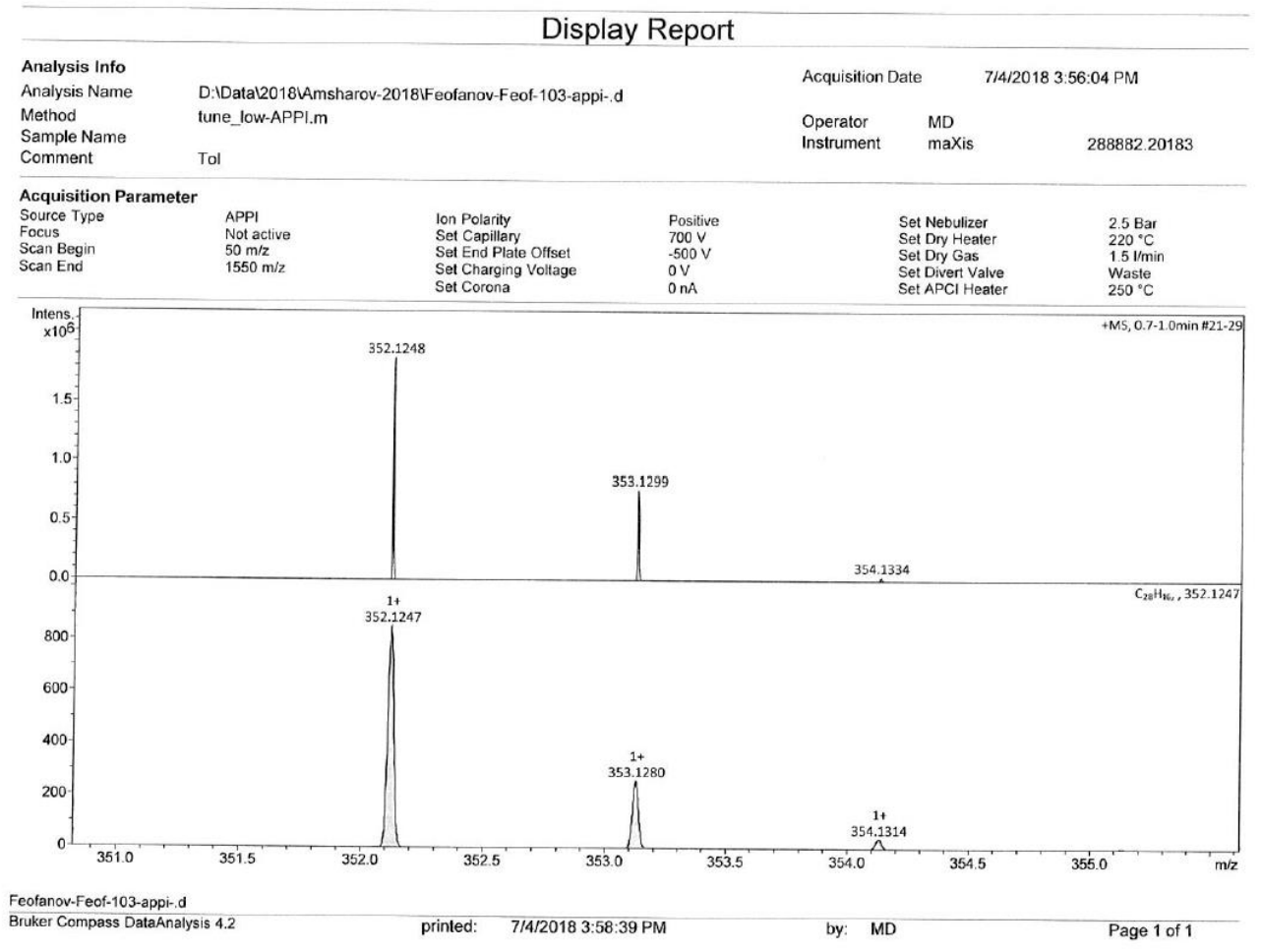

Figure S51. APPI-HRMS spectrum of NG1.

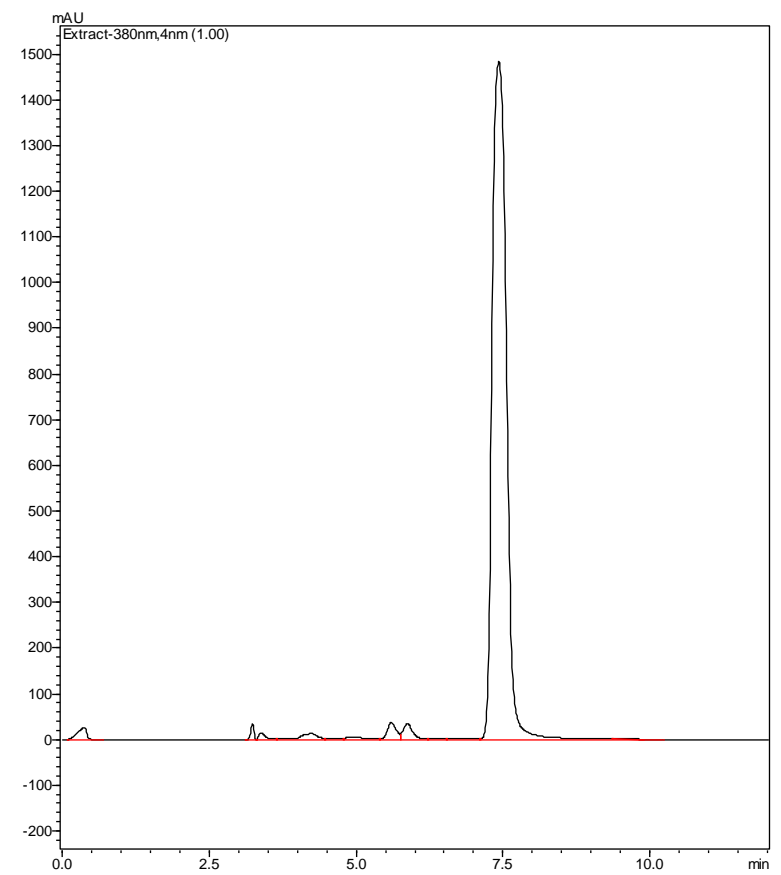

Figure S52. HPLC profile of NG1 after column chromatography; detected at $380 \mathrm{~nm}$ (PBr column, toluene-MeOH 1-1 as eluent, $\left.1 \mathrm{~mL} / \mathrm{min}, 40^{\circ} \mathrm{C}\right)$. 

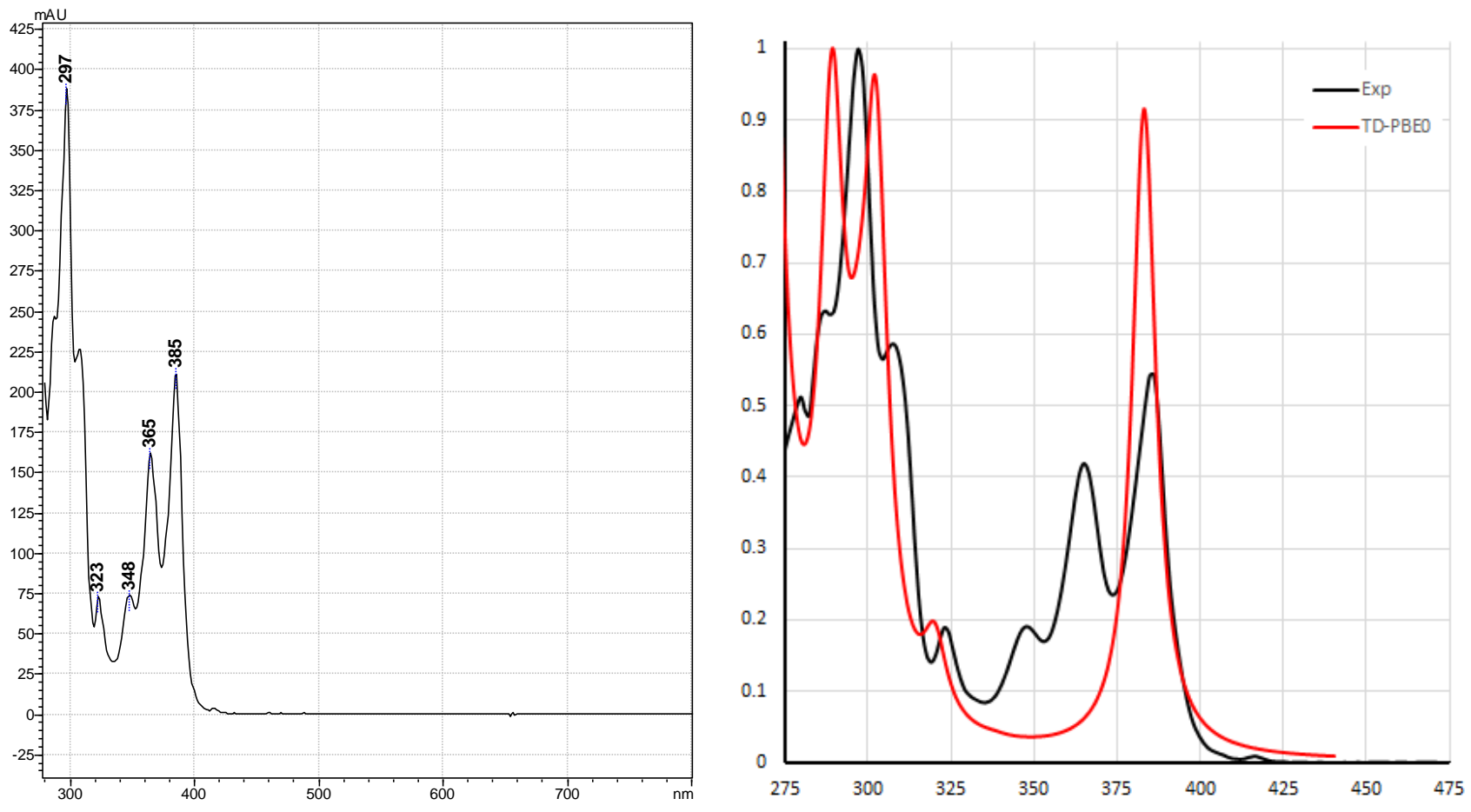

Figure S53. Left side: UV-Vis spectrum of NG1 (toluene-MeOH 1-1) from HPLC. Right side: Comparison of experimental (black) and predicted by TD-PBE0 UV/Vis spectra (red).

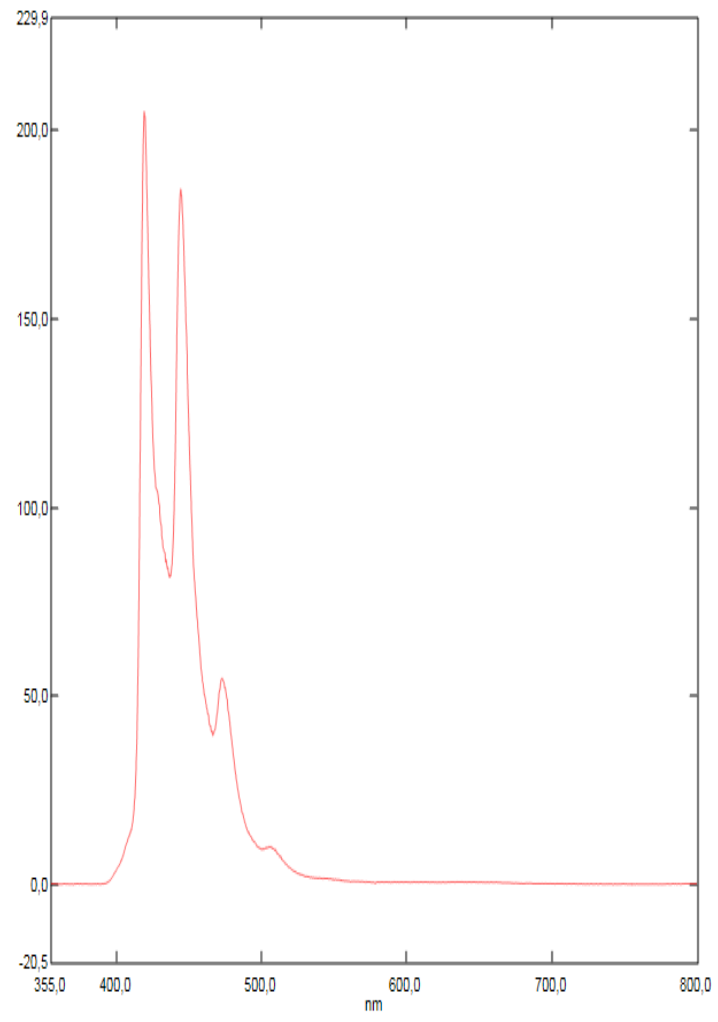

Figure S54. Fluorescence spectrum of NG1 (DCM). 


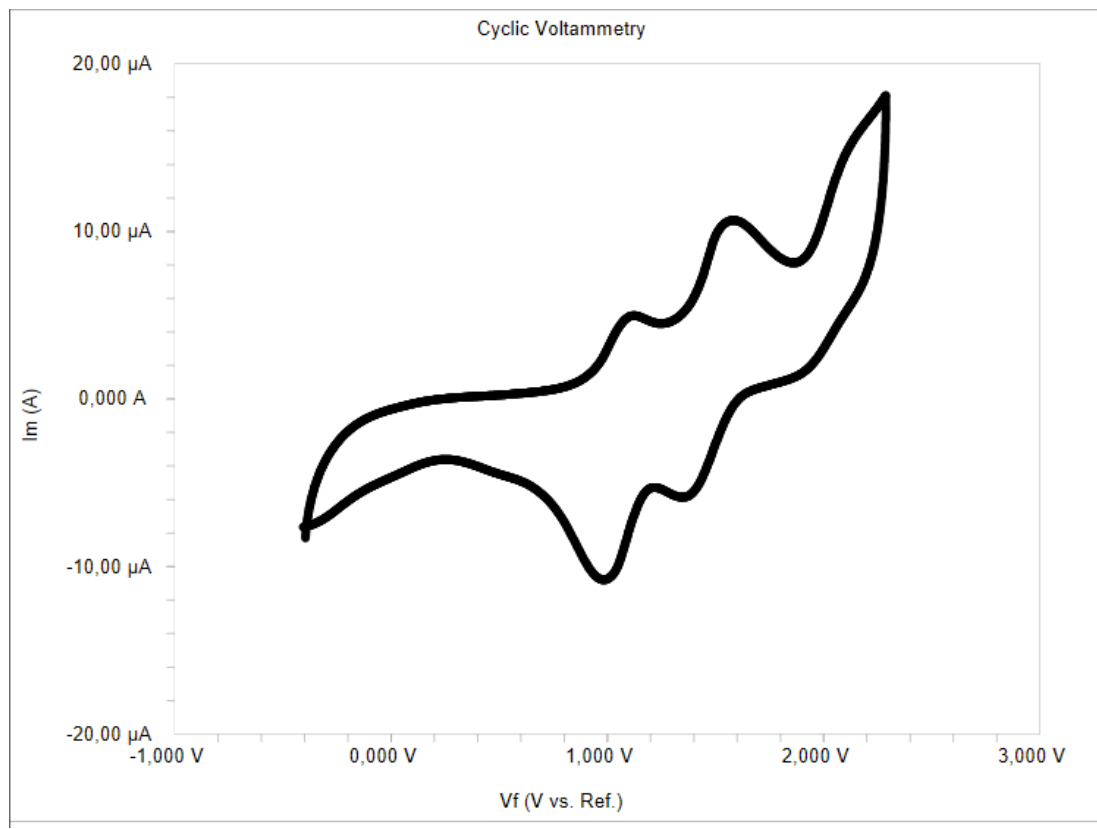

Figure S55. Cyclic voltammogram of NG1 measured in $0.1 \mathrm{M} \mathrm{Bu}_{4} \mathrm{NPF}_{6}$ in 1,2-dichlorobenzene at room temperature with $100 \mathrm{mV} / \mathrm{s}$ scan speed.
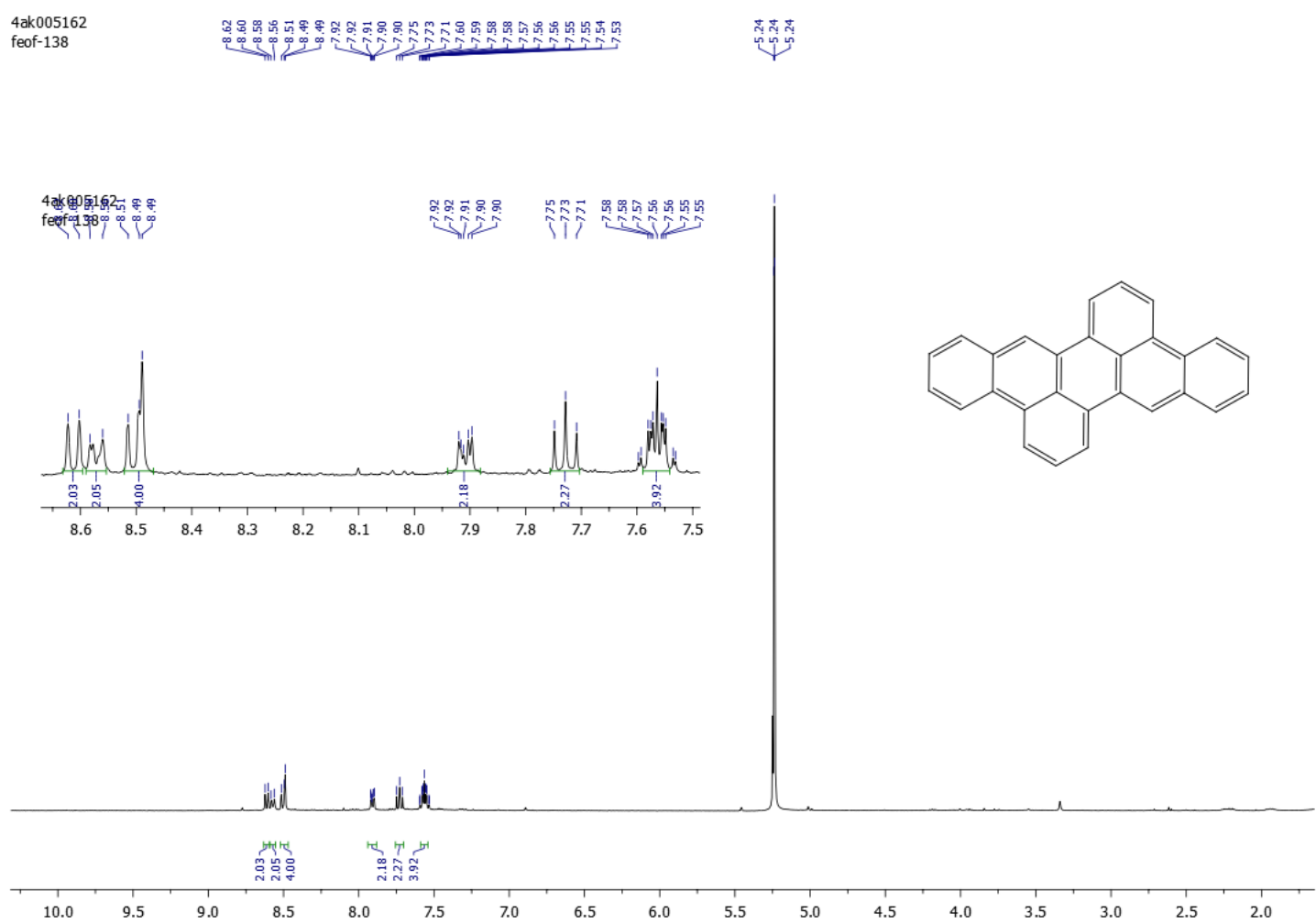

Figure S56. ${ }^{1} \mathrm{H}$ NMR (400 MHz, $\left.\mathrm{CD}_{2} \mathrm{Cl}_{2}, 293 \mathrm{~K}\right)$ spectrum of NG2. 


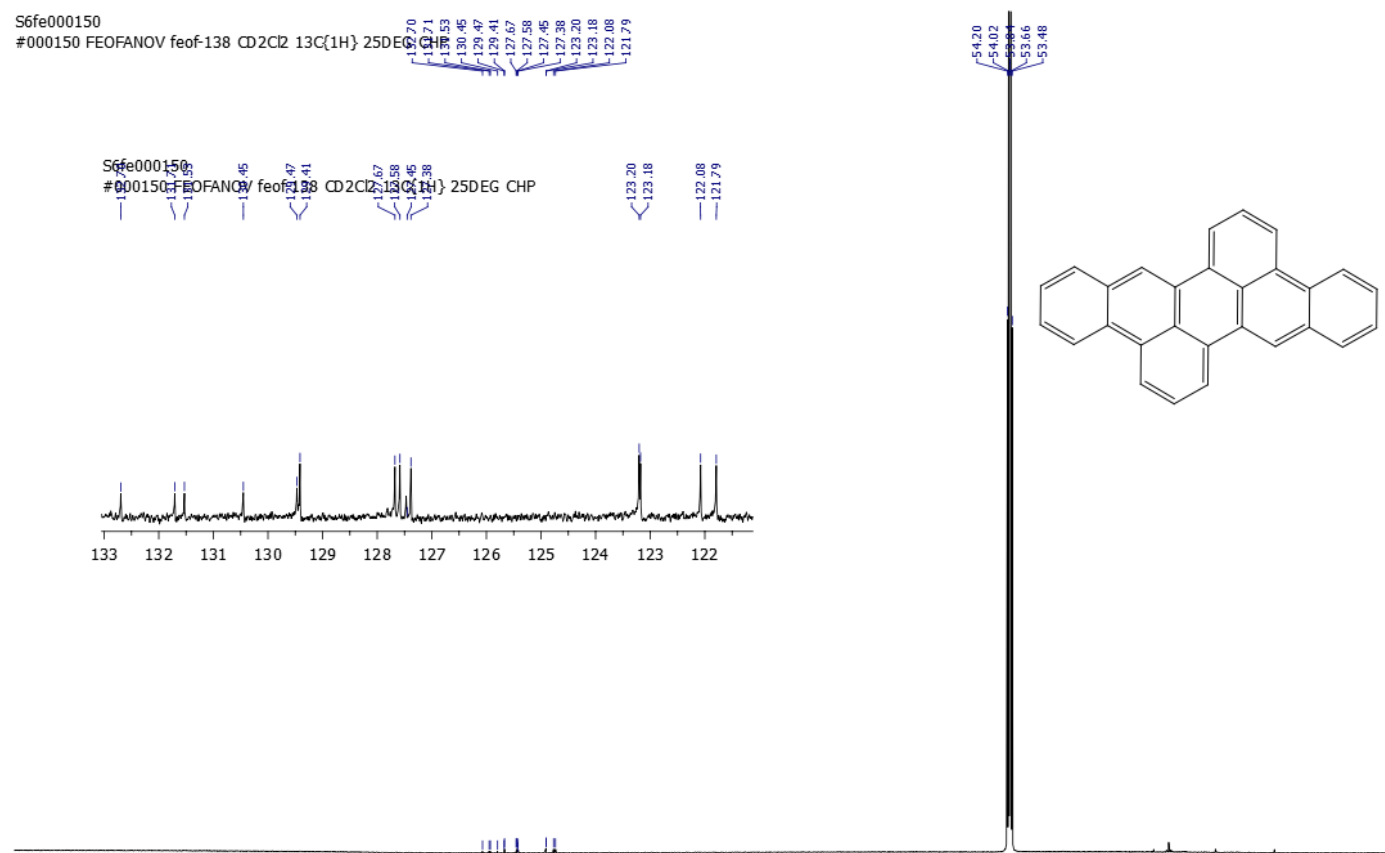

Figure S57. ${ }^{13} \mathrm{C}$ NMR $\left(150 \mathrm{MHz}, \mathrm{CD}_{2} \mathrm{Cl}_{2}, 293 \mathrm{~K}\right)$ spectrum of NG2.

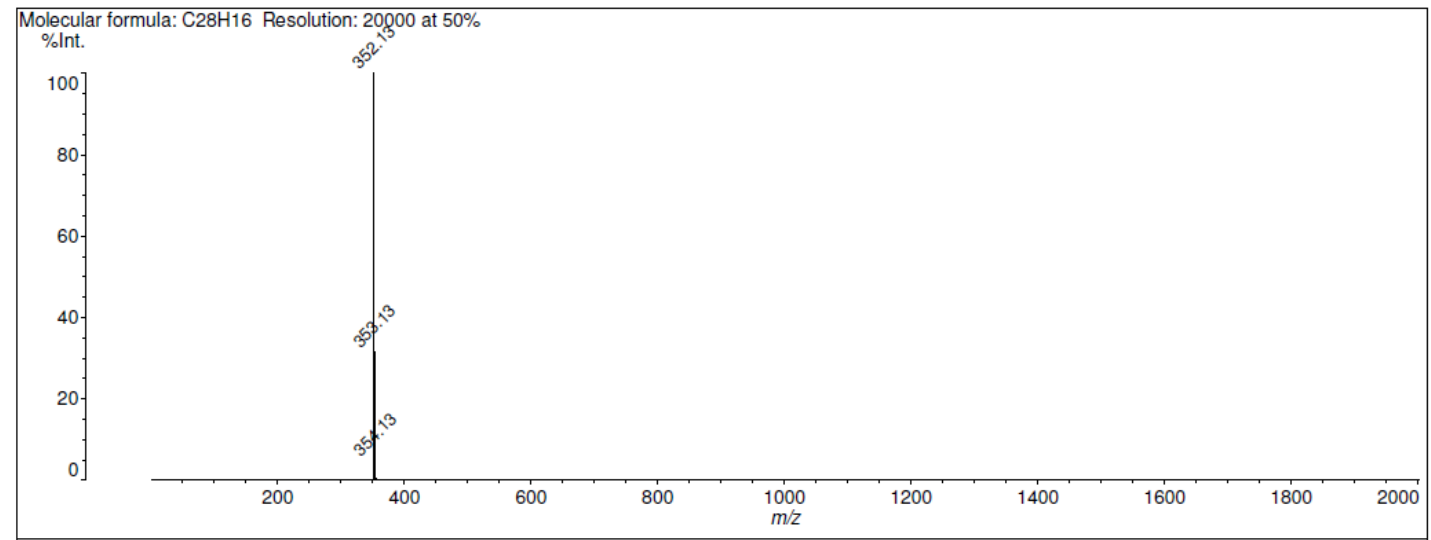

Data: C:IDocuments and SettingsIMALDIDesktoplDatalFeofanovifeof-138_10001.P9[c] 31 Jul 2018 12:38 Cal: PAH3 3 Jul 2018 16:32 Shimadzu Biotech Axima Confidence 2.8.5.20090629: Mode Reflectron, Power: 84, Blanked, P.Ext. @ 380 (bin 52)

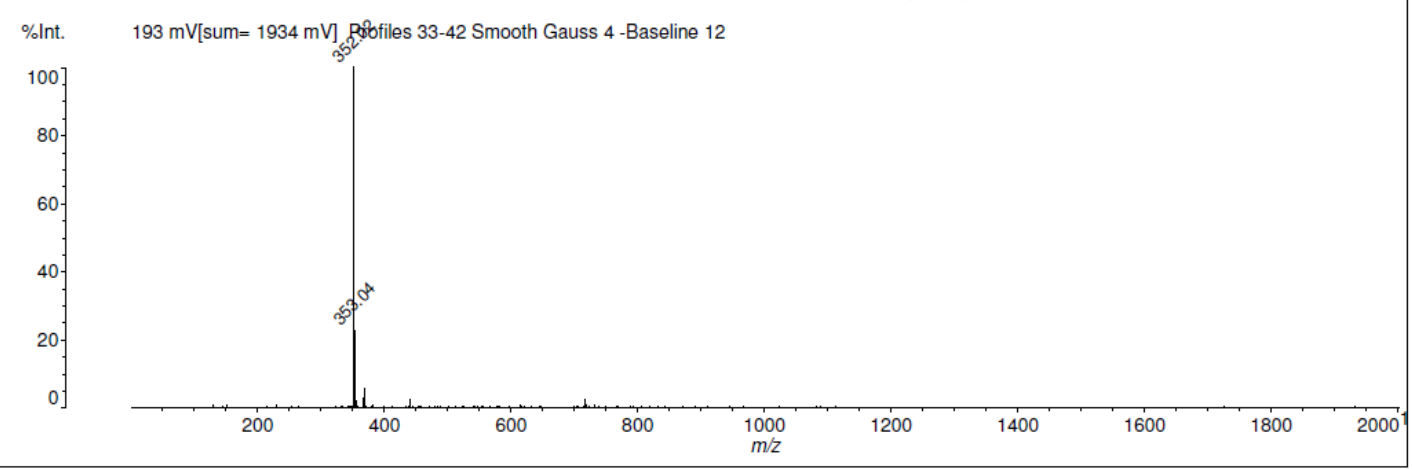

Figure S58. LDI-TOF MS spectrum of NG2. 


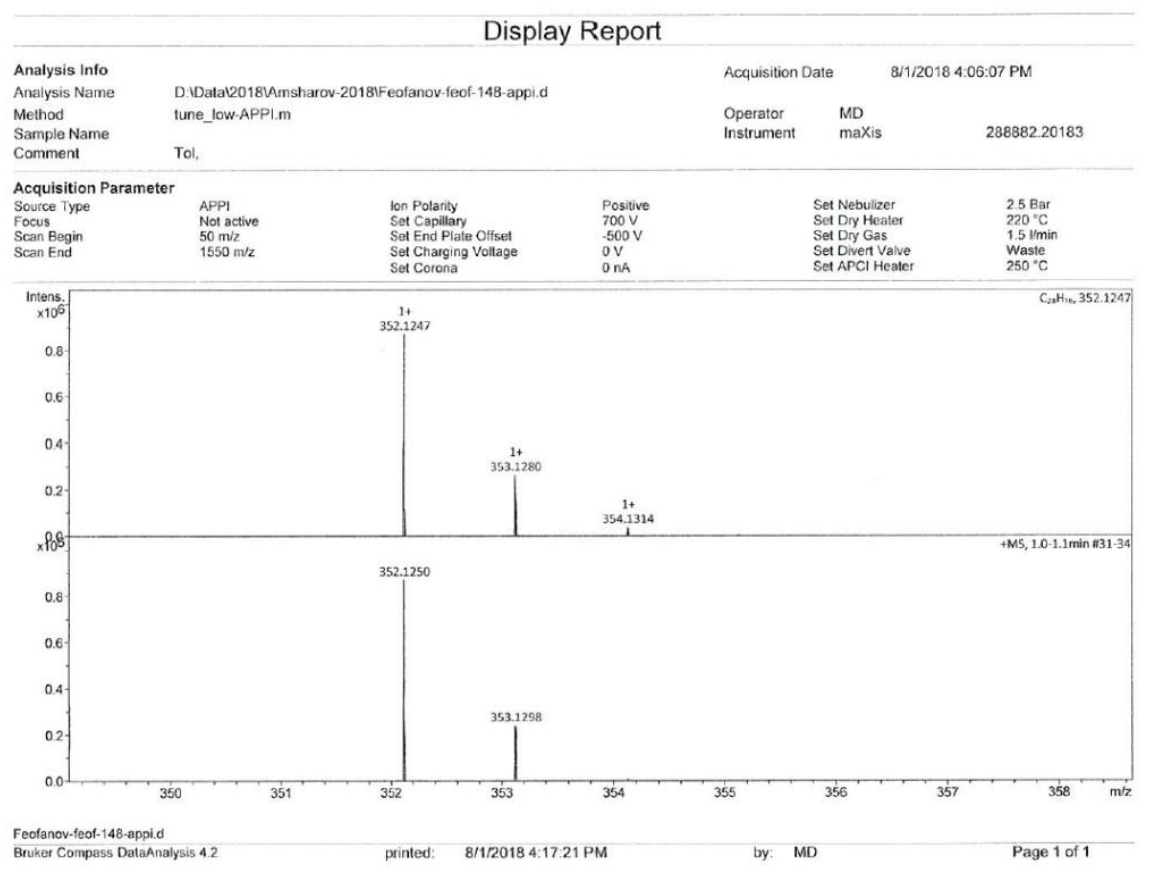

Figure S59. APPI-HRMS spectrum of NG2.

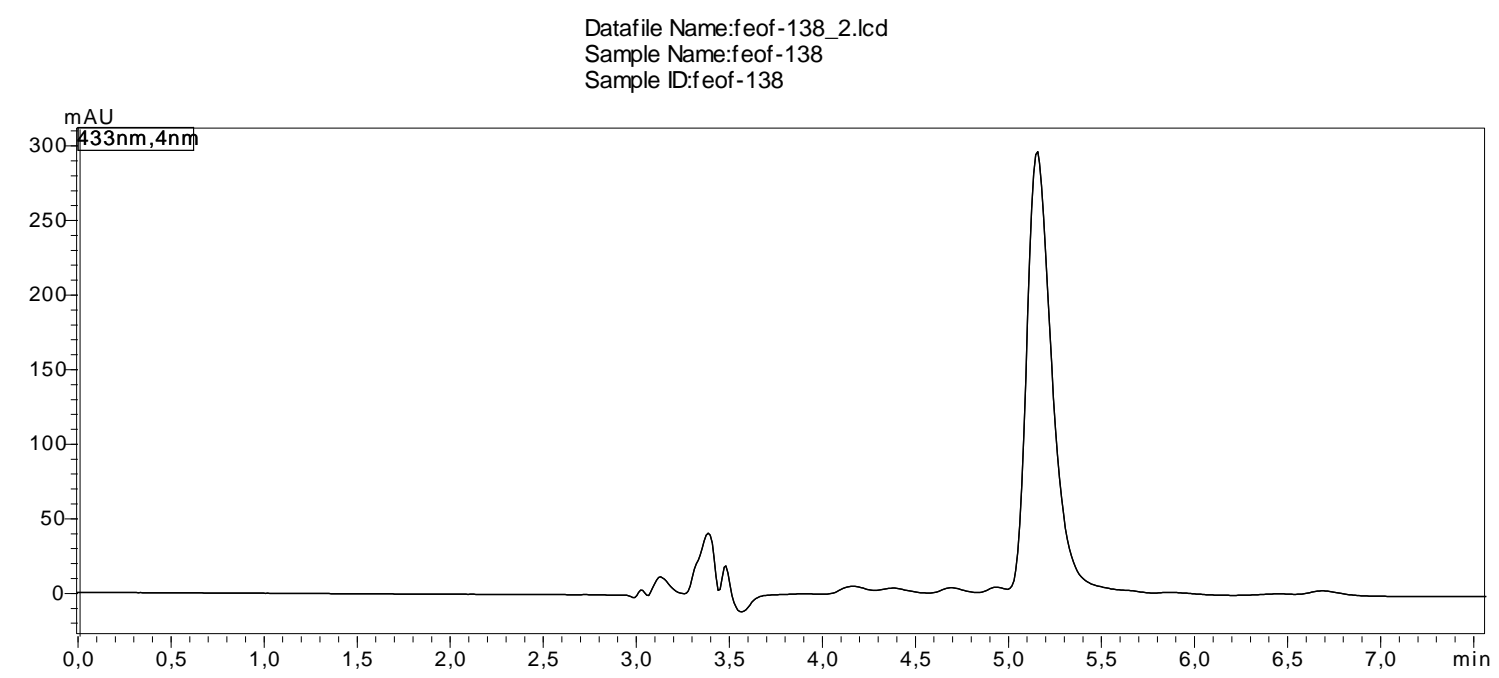

Figure S60. HPLC profile of NG2 after column chromatography; detected at $435 \mathrm{~nm}$ ( $\mathrm{PBr}$ column, toluene-MeOH $4-1$ as eluent, $1 \mathrm{~mL} / \mathrm{min}, 40^{\circ} \mathrm{C}$ ). 

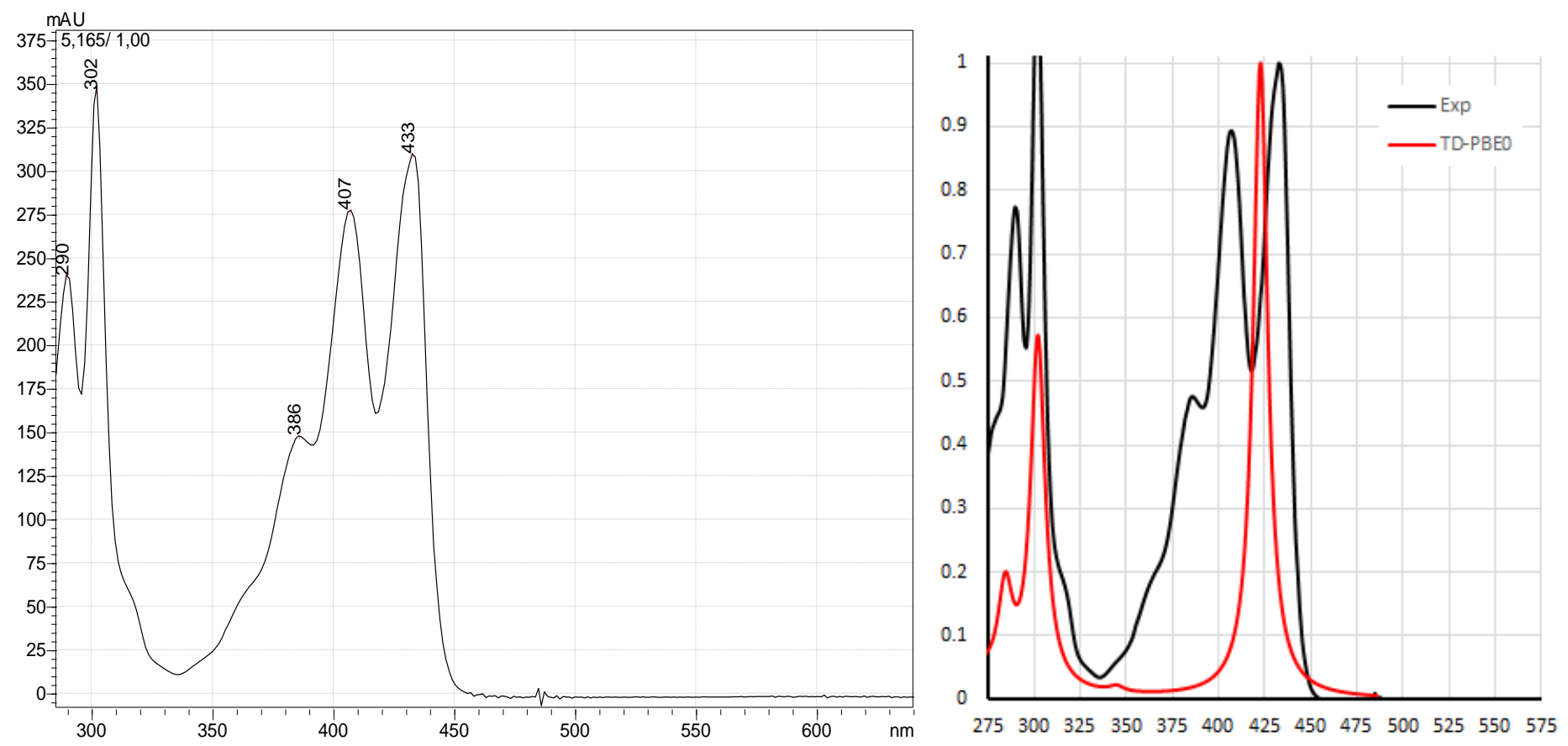

Figure S61. Left side: UV-Vis spectrum of NG2 (toluene-MeOH 4-1) from HPLC. Right side: Comparison of experimental (black) and predicted by TD-PBE0 UV/Vis spectra (red)

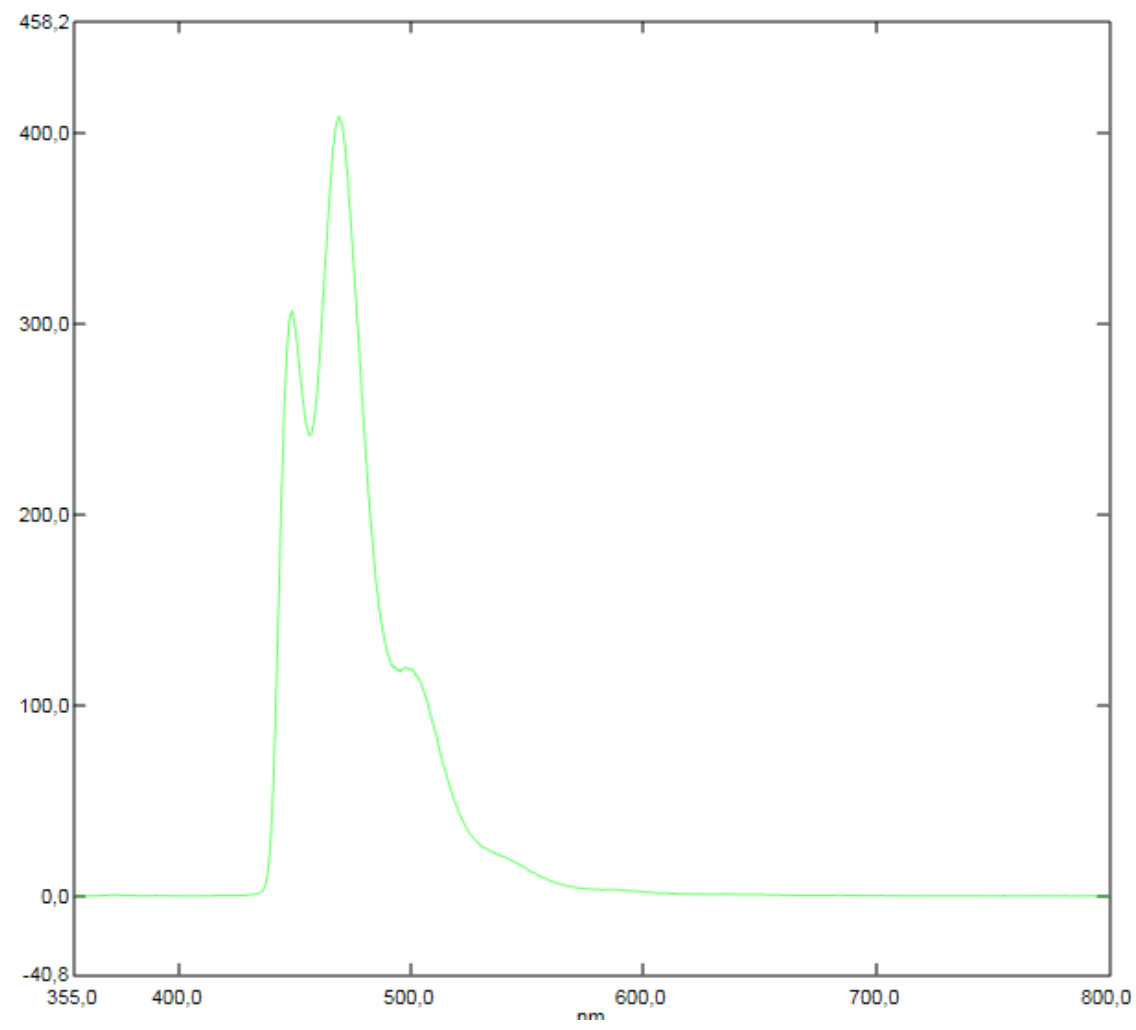

Figure S62. Fluorescence spectrum of NG1 (DCM). 


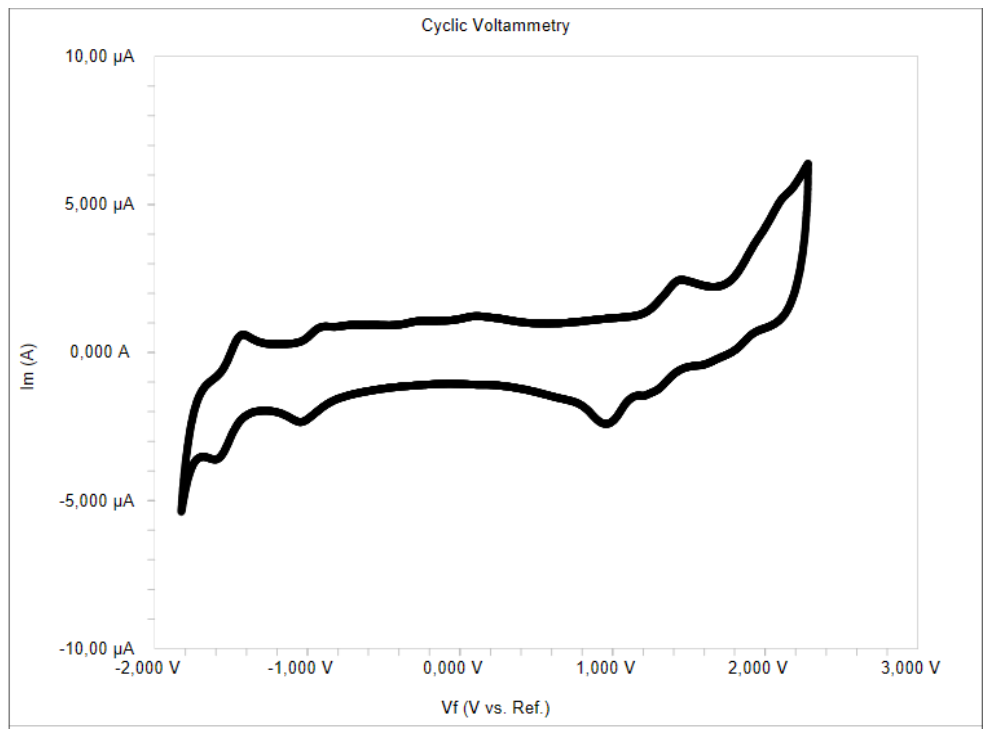

Figure S63. Cyclic voltammogram of NG2 measured in $0.1 \mathrm{M} \mathrm{Bu}_{4} \mathrm{NPF}_{6}$ in 1,2-dichlorobenzene at room temperature with $100 \mathrm{mV} / \mathrm{s}$ scan speed.

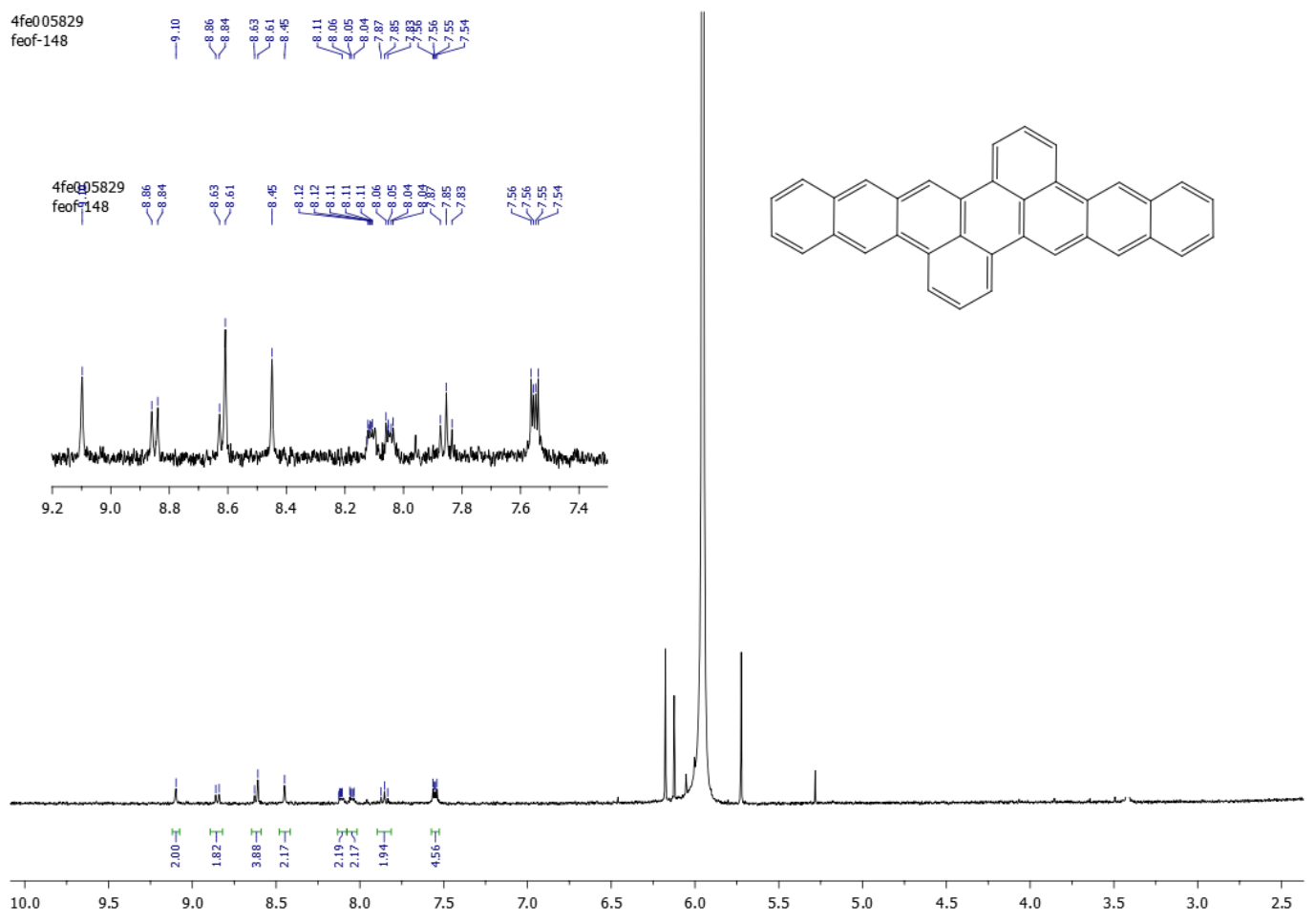

Figure S64. ${ }^{1} \mathrm{H} \mathrm{NMR}\left(400 \mathrm{MHz}, \mathrm{C}_{2} \mathrm{D}_{2} \mathrm{Cl}_{4}, 293 \mathrm{~K}\right)$ spectrum of NG3. 


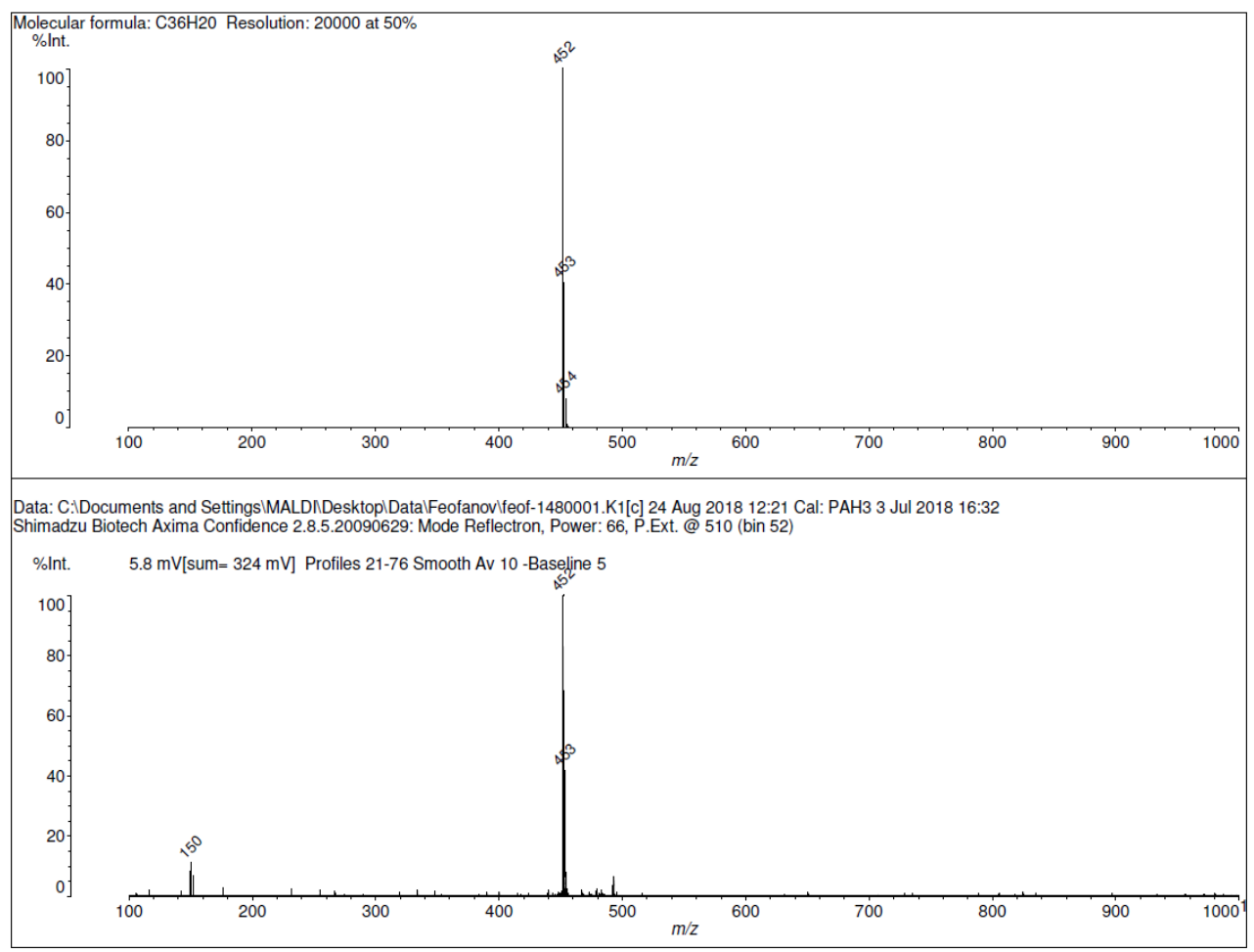

Figure S65. LDI-TOF MS spectrum of NG3.

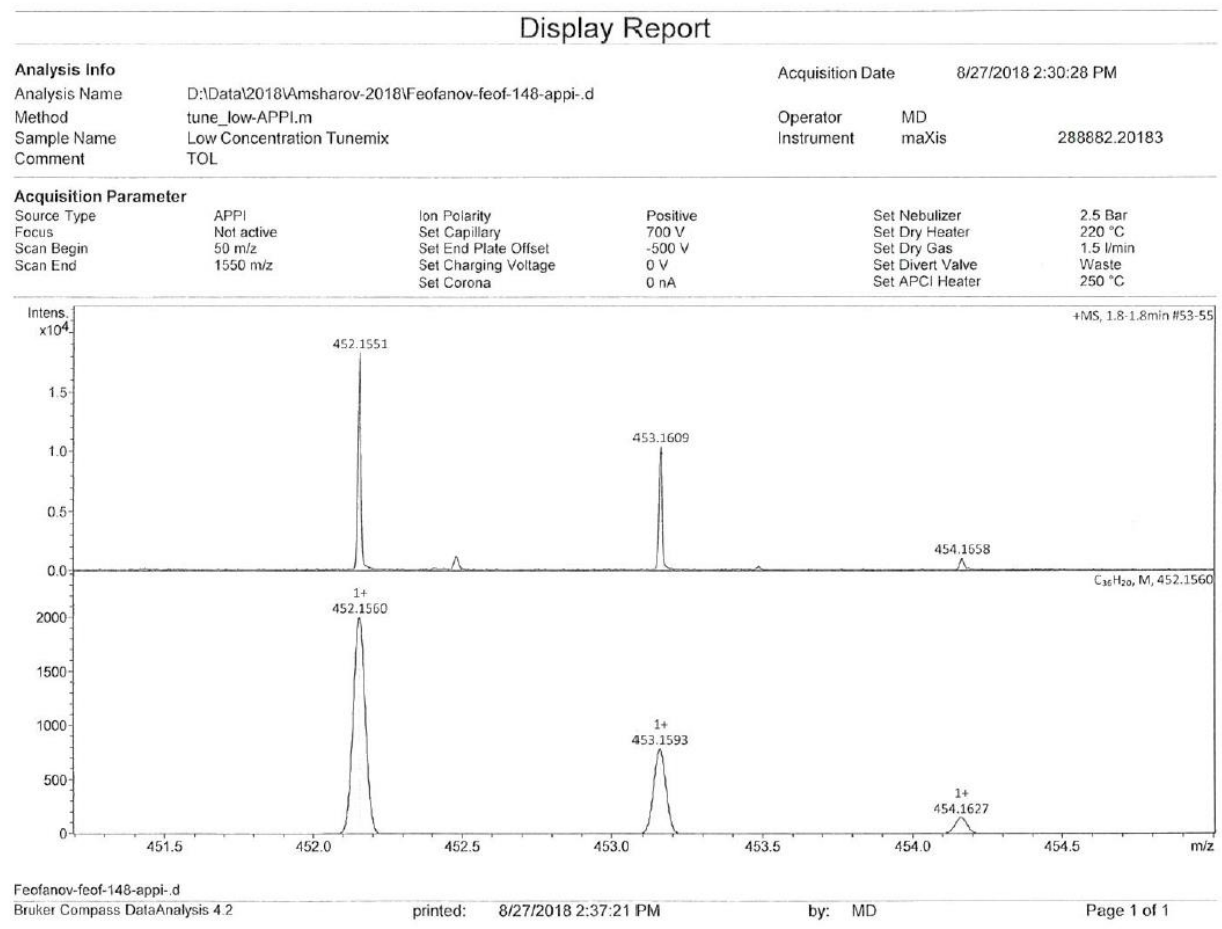

Figure S66. APPI-HRMS spectrum of NG3. 
Datafile Name:feof-148 1.Icd

Sample Name:feof-148

Sample ID:feof-148

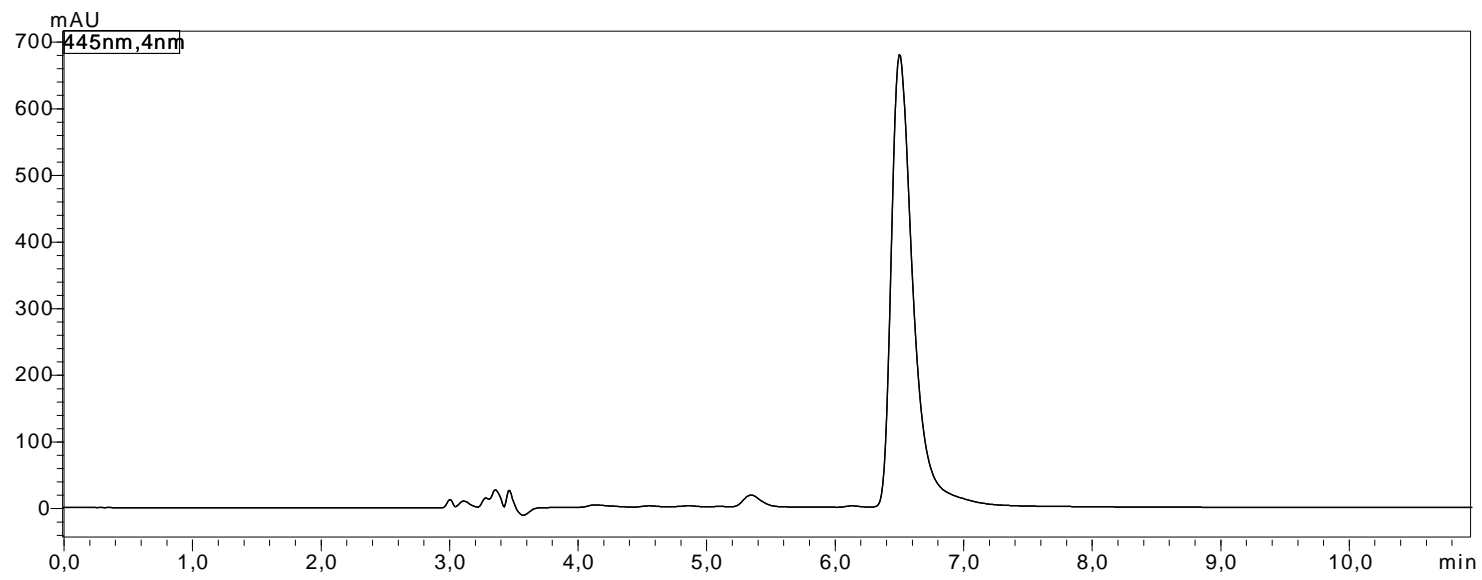

Figure S67. HPLC profile of NG3 after aqueous work-up; detected at $443 \mathrm{~nm}$ ( $\mathrm{PBr}$ column, toluene/MeOH 4-1, $\left.1 \mathrm{~mL} / \mathrm{min}, 40^{\circ} \mathrm{C}\right)$.
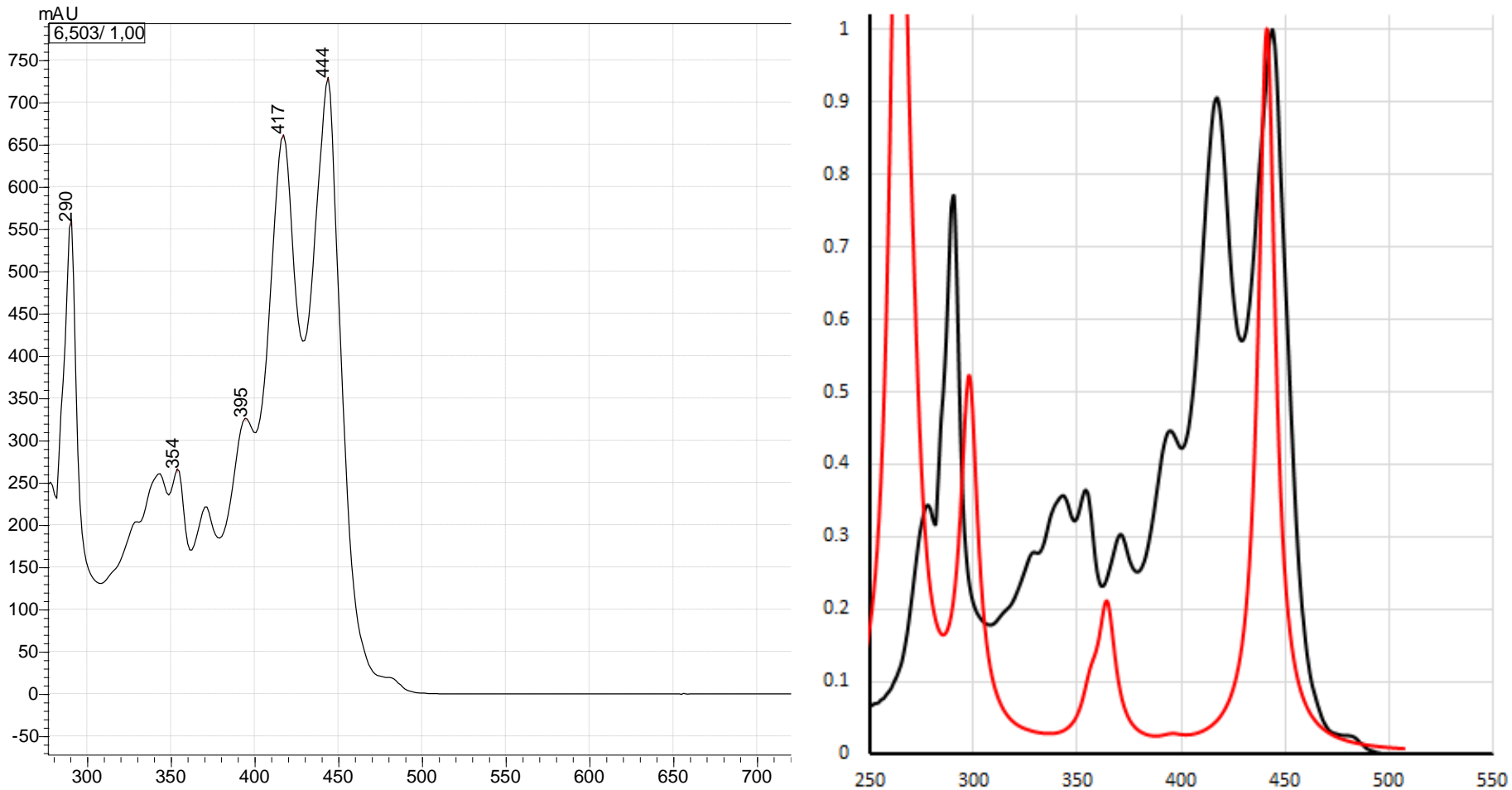

Figure S68. Left side: UV-Vis spectrum of NG3 (toluene-MeOH 4-1) from HPLC. Right side: Comparison of experimental (black) and predicted by TD-PBE0 UV/Vis spectra (red). 


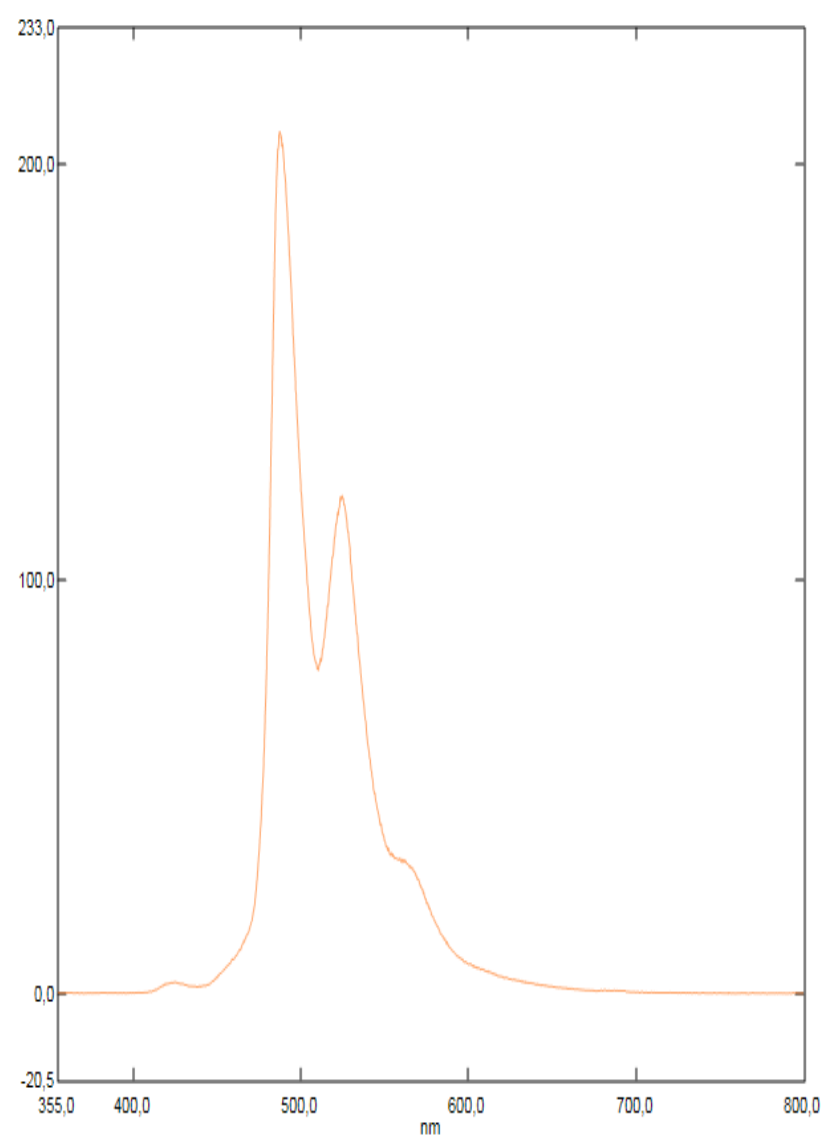

Figure S69. Fluorescence spectrum of NG3 (DCM).

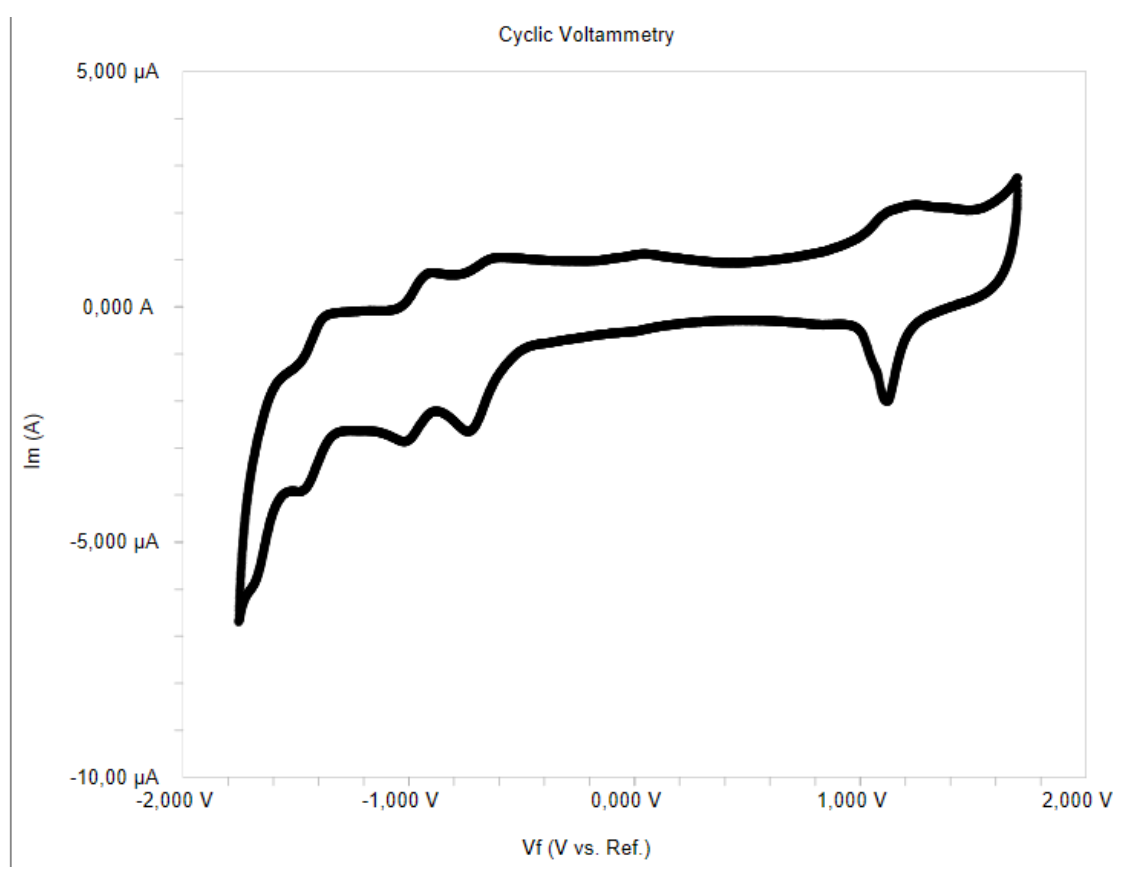

Figure S70. Cyclic voltammogram of NG3 measured in $0.1 \mathrm{M} \mathrm{Bu}_{4} \mathrm{NPF}_{6}$ in 1,2-dichlorobenzene at room temperature with $100 \mathrm{mV} / \mathrm{s}$ scan speed. 


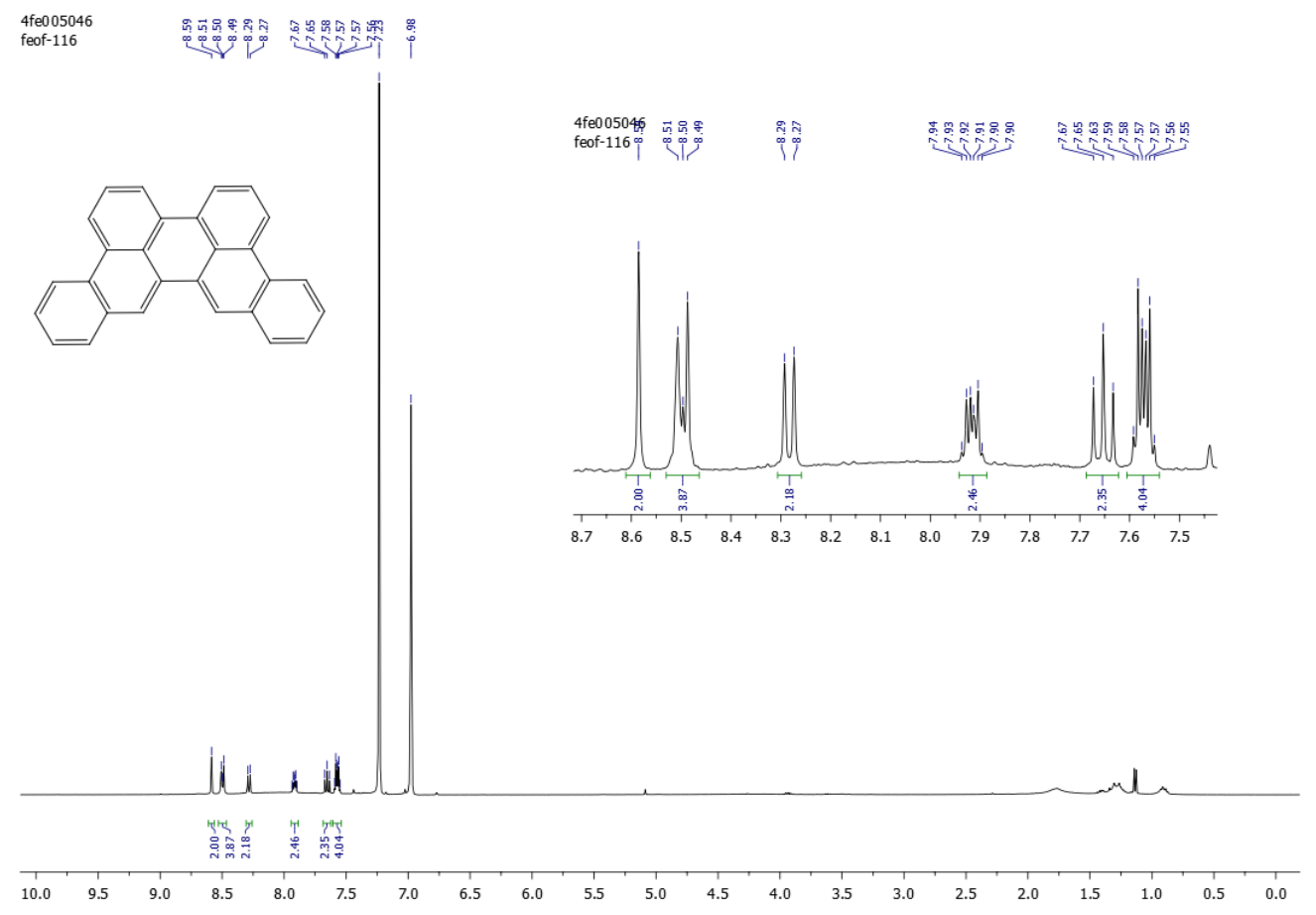

Figure S71. ${ }^{1} \mathrm{H}$ NMR (400 MHz, $\left.o-\mathrm{C}_{6} \mathrm{D}_{4} \mathrm{Cl}_{2}, 293 \mathrm{~K}\right)$ spectrum of NG4.
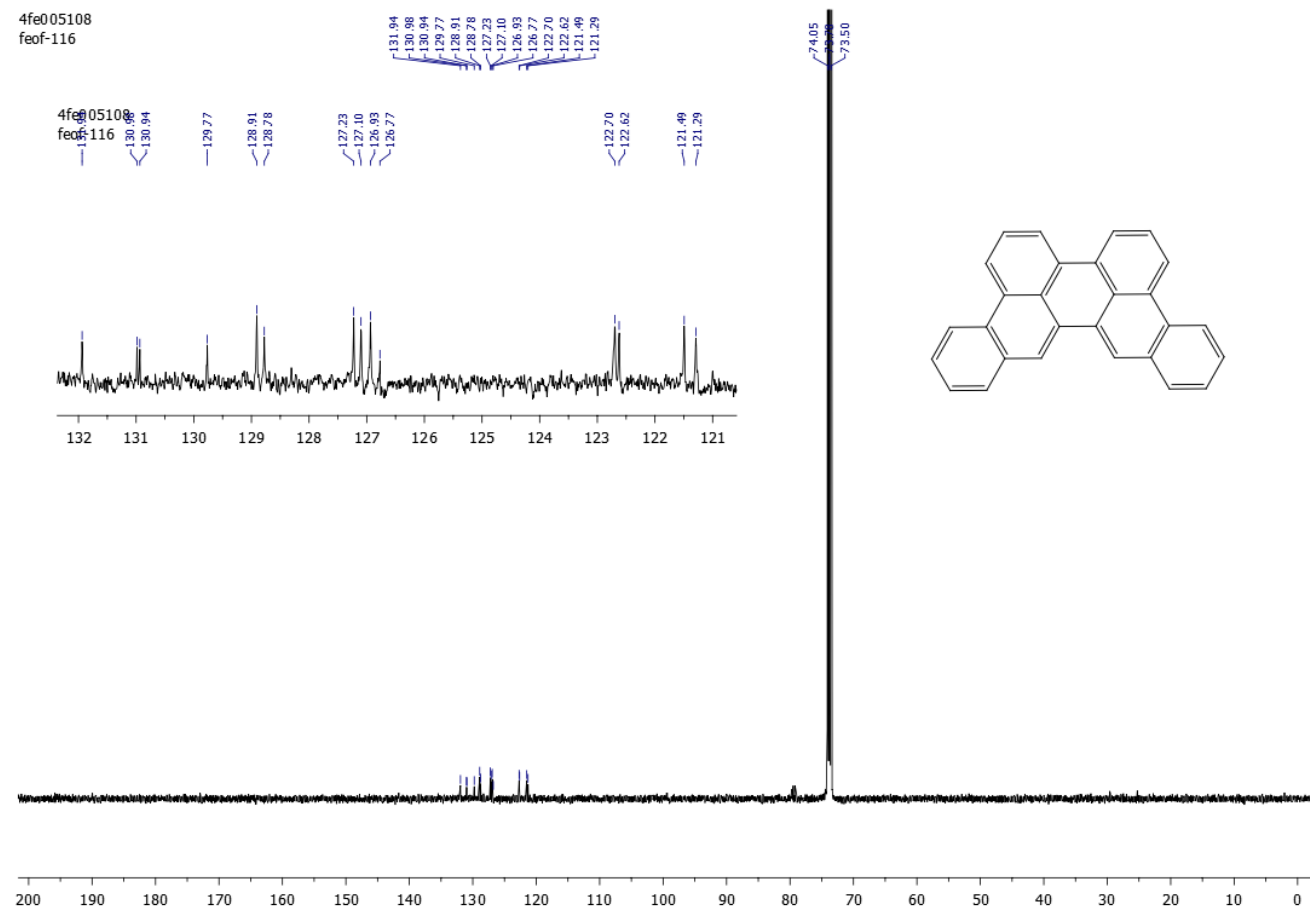

Figure S72. ${ }^{13} \mathrm{C}$ NMR (101 MHz, $\left.\mathrm{C}_{2} \mathrm{D}_{2} \mathrm{Cl}_{4}, 293 \mathrm{~K}\right)$ spectrum of NG4. 


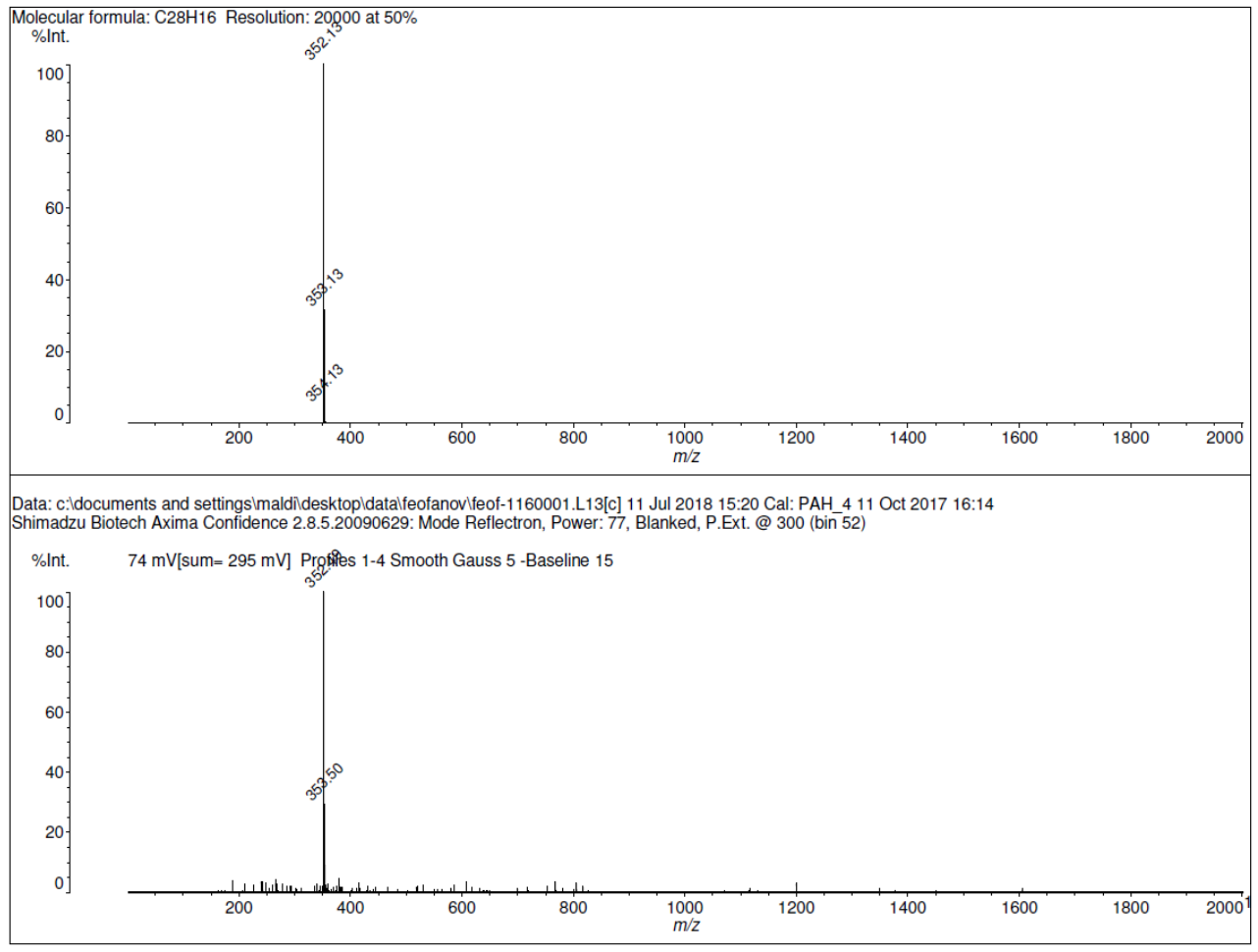

Figure S73. LDI-TOF MS spectrum of NG4.

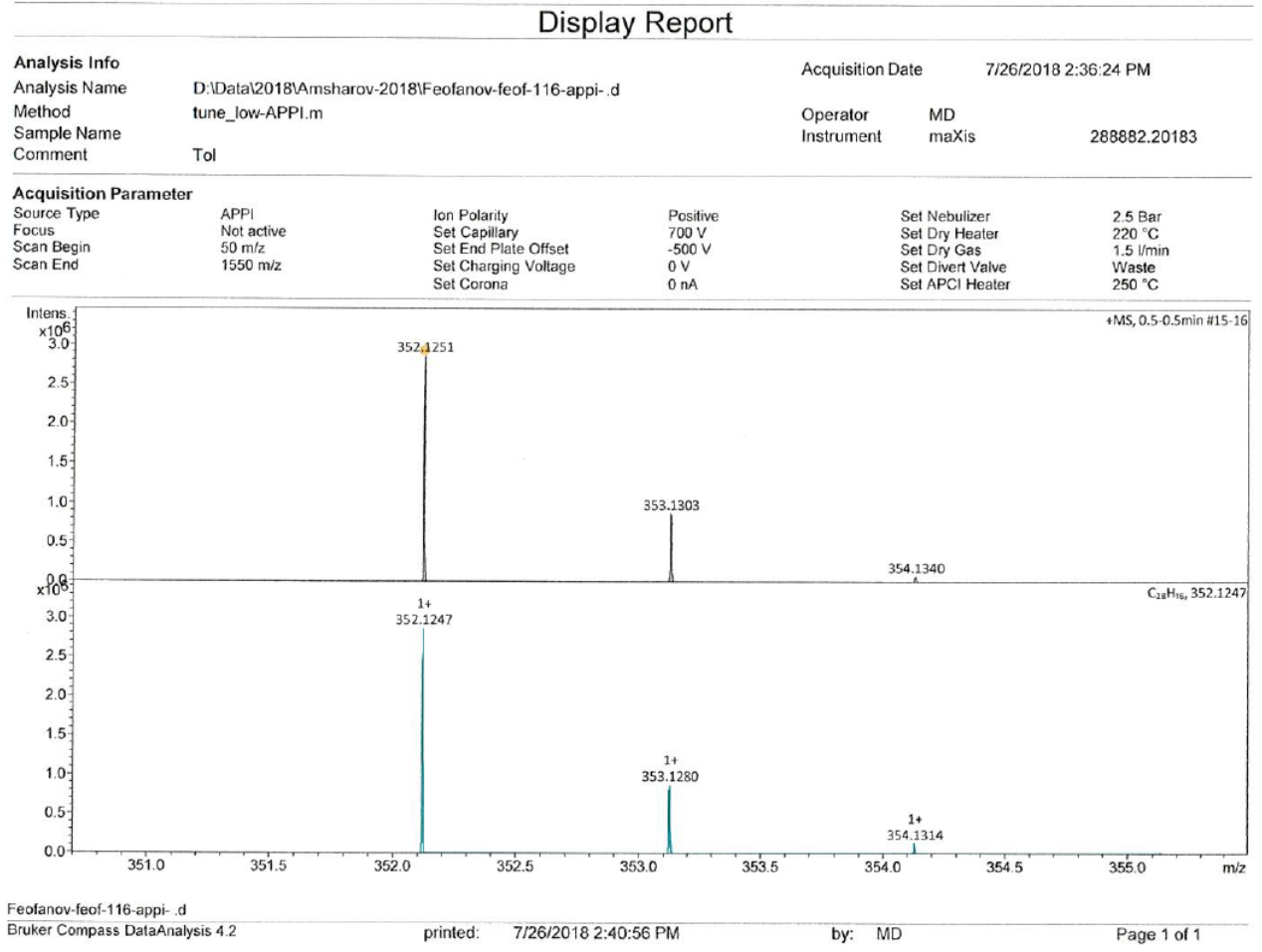

Figure S74. APPI-HRMS spectrum of NG4. 


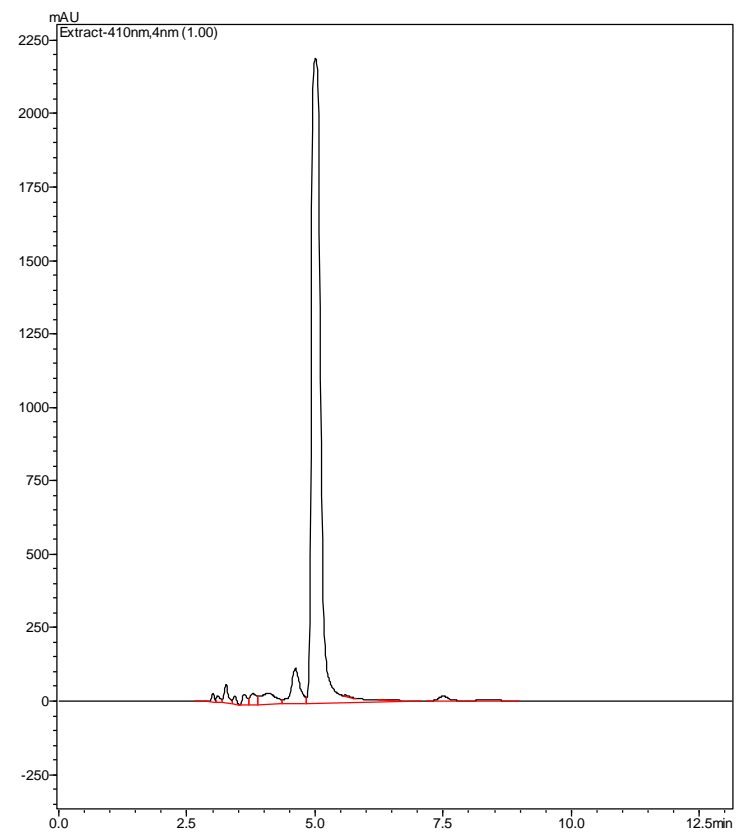

Figure S75. HPLC profile of NG4 after aqueous work-up; detected at $410 \mathrm{~nm}$ (PBr column, toluene$\mathrm{MeOH} 1-1$ as eluent, $1 \mathrm{~mL} / \mathrm{min}, 40^{\circ} \mathrm{C}$ ).
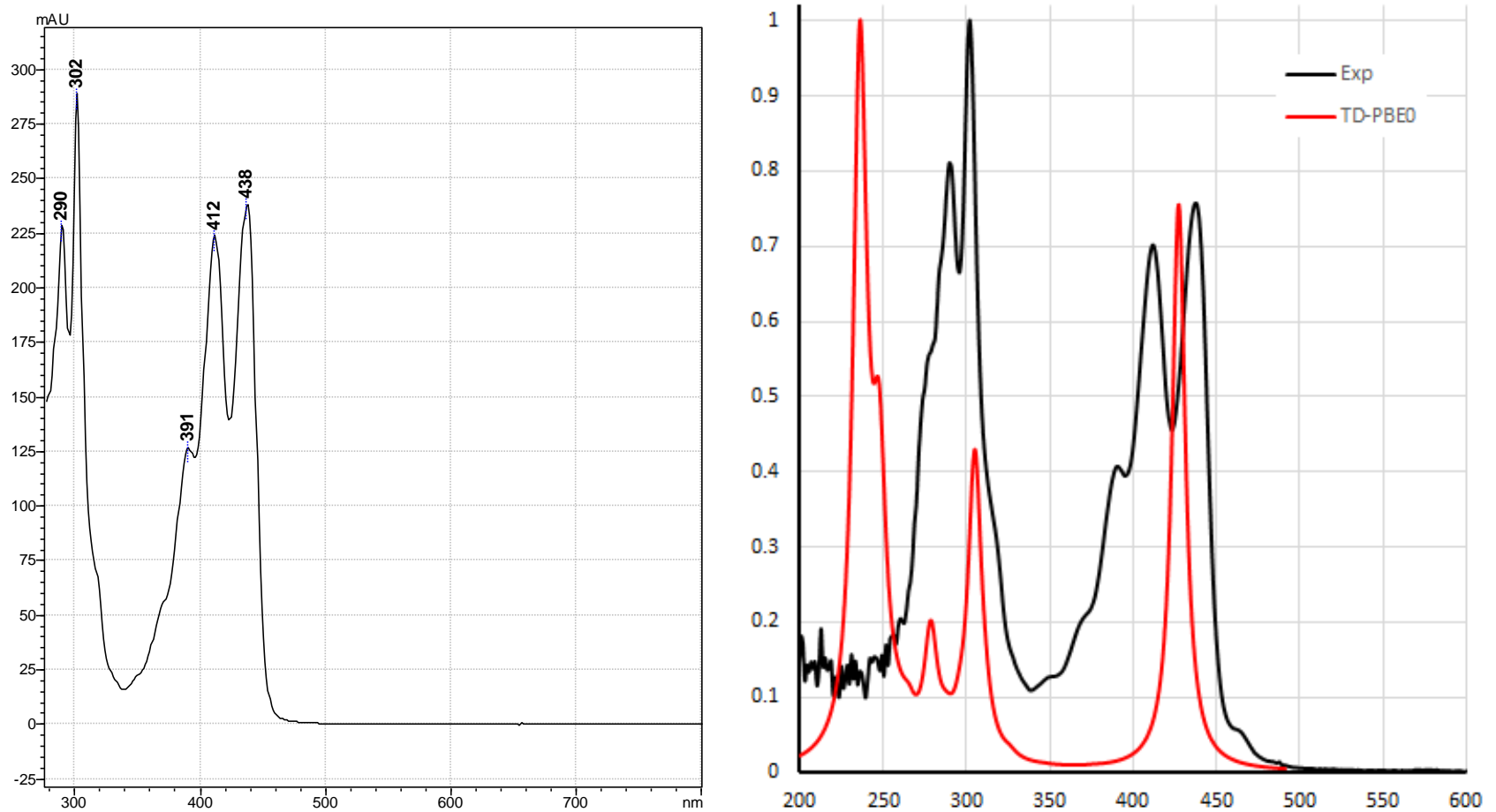

Figure S76. Left side: UV-Vis spectrum of NG4 (toluene-MeOH 4-1) from HPLC. Right side: Comparison of experimental (black) and predicted by TD-PBE0 UV/Vis spectra (red). 


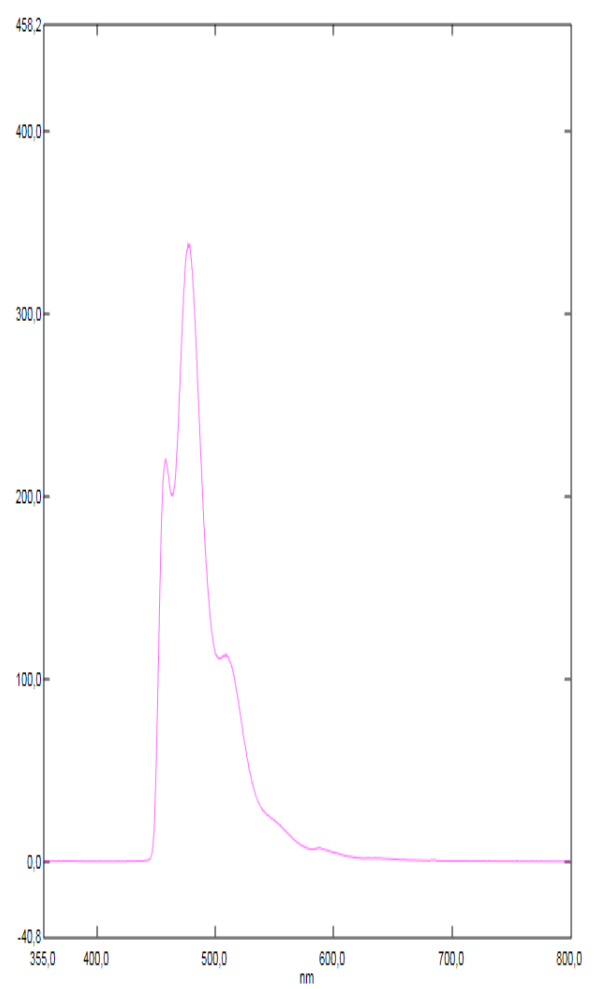

Figure S77. Fluorescence spectrum of NG4 (DCM).

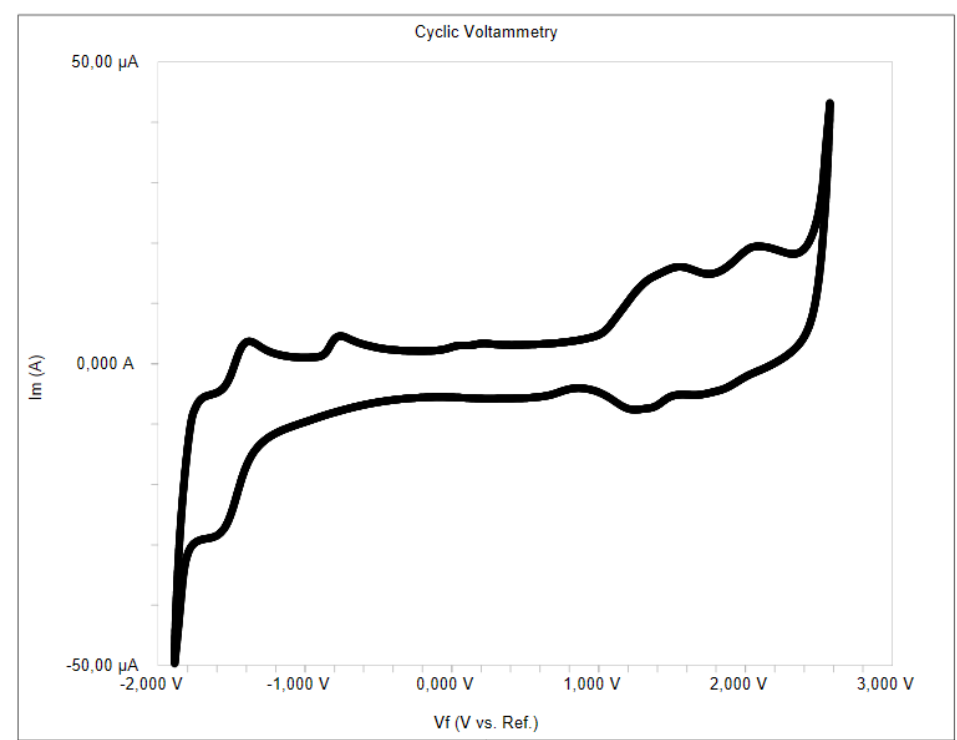

Figure S78. Cyclic voltammogram of NG4 measured in $0.1 \mathrm{M} \mathrm{Bu}_{4} \mathrm{NPF}_{6}$ in 1,2-dichlorobenzene at room temperature with $100 \mathrm{mV} / \mathrm{s}$ scan speed. 


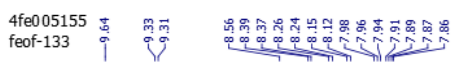

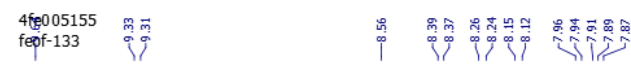

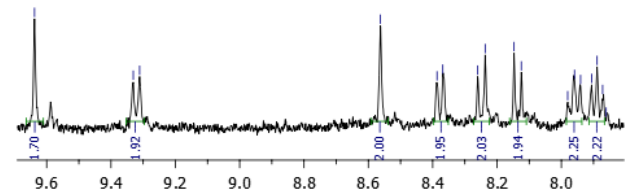

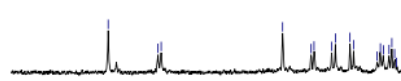

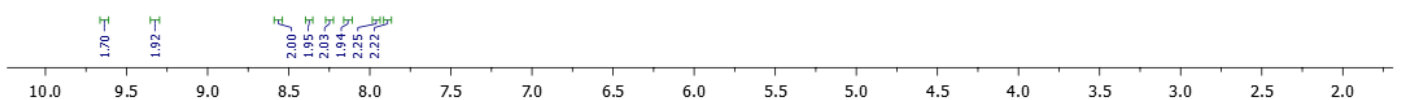

Figure S79. ${ }^{1} \mathrm{H}$ NMR (400 MHz, $\left.\mathrm{CD}_{2} \mathrm{Cl}_{2}, 293 \mathrm{~K}\right)$ spectrum of NG5.

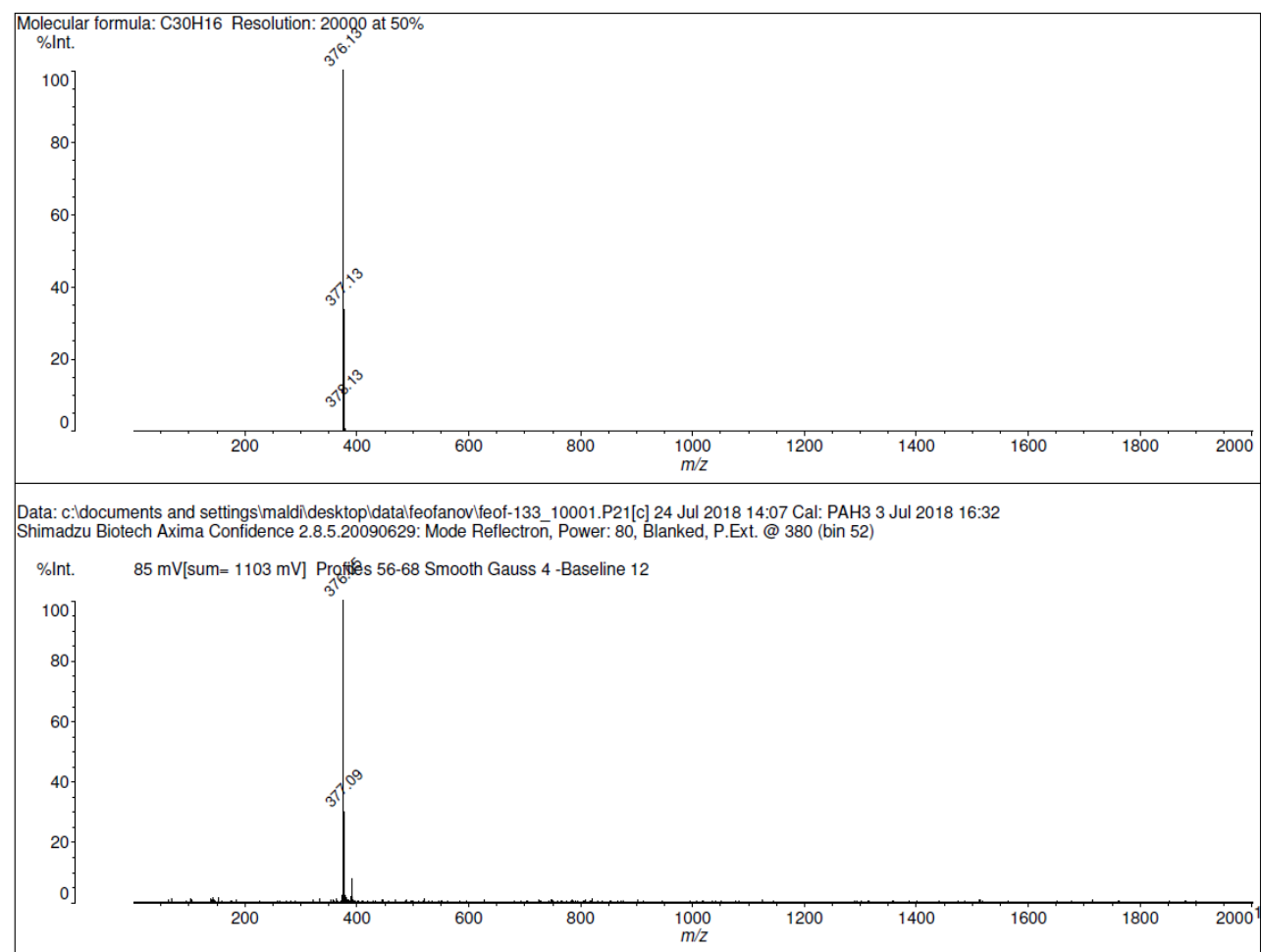

Figure S80. LDI-TOF MS spectrum of NG5. 


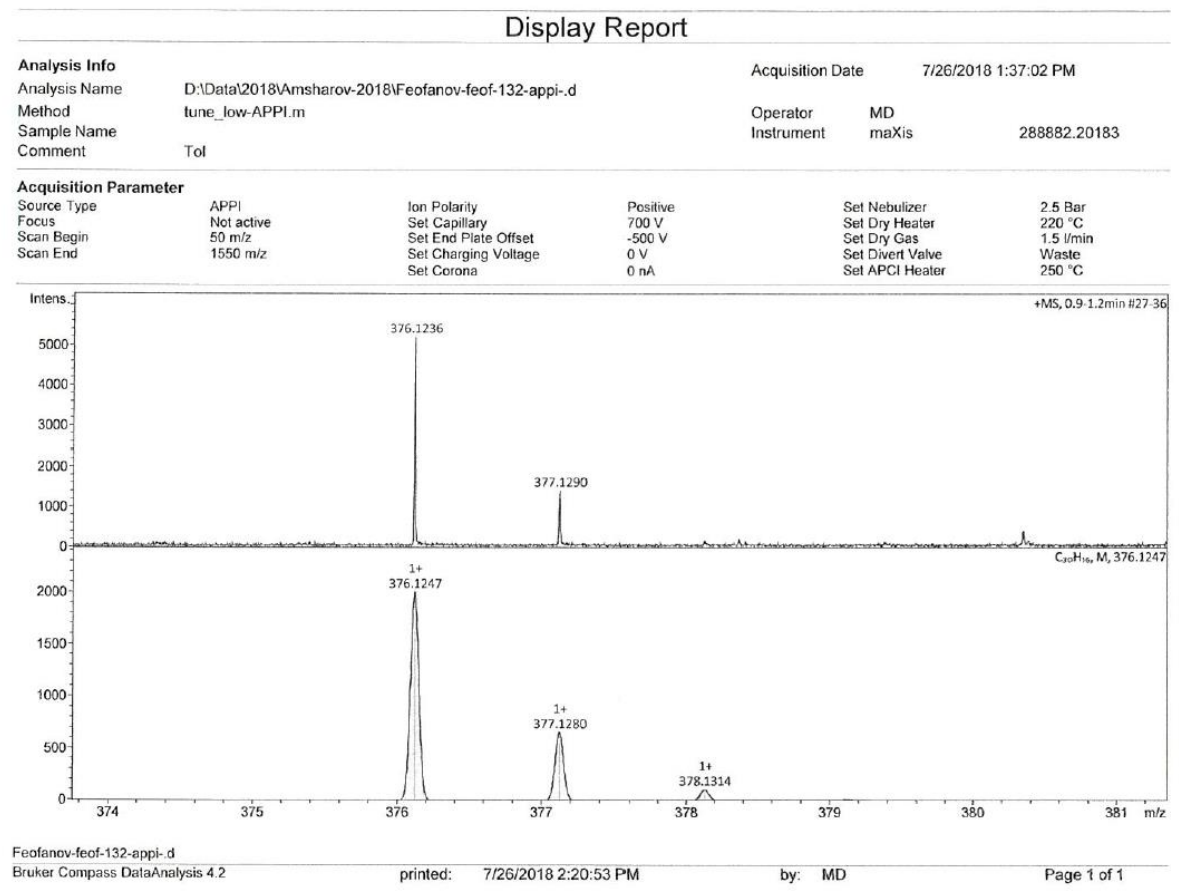

Figure S81. APPI-HRMS spectrum of NG5.

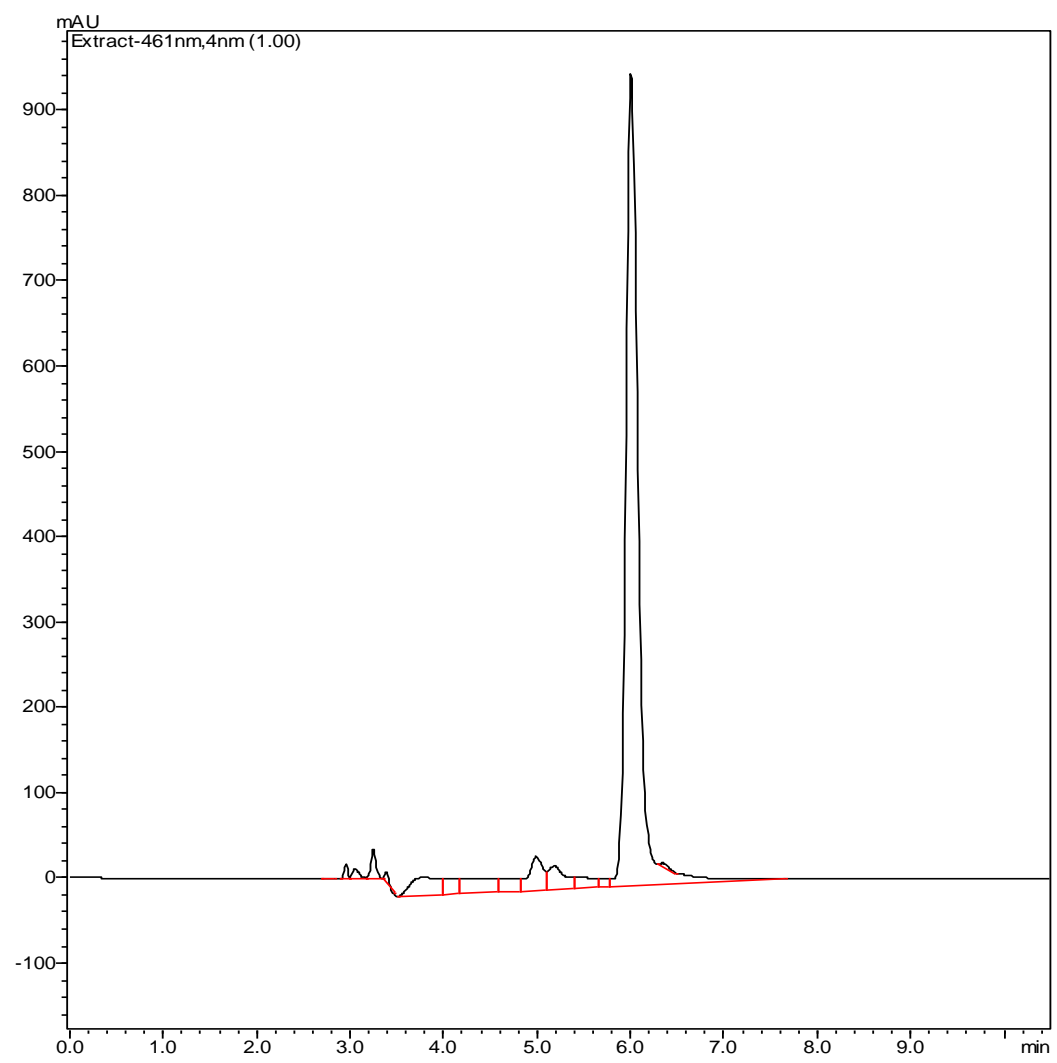

Figure S82. HPLC profile of NG5 after aqueous work-up; detected at $461 \mathrm{~nm}$ (PBr column, toluene$\mathrm{MeOH} 4-1$ as eluent, $1 \mathrm{~mL} / \mathrm{min}, 40^{\circ} \mathrm{C}$ ). 

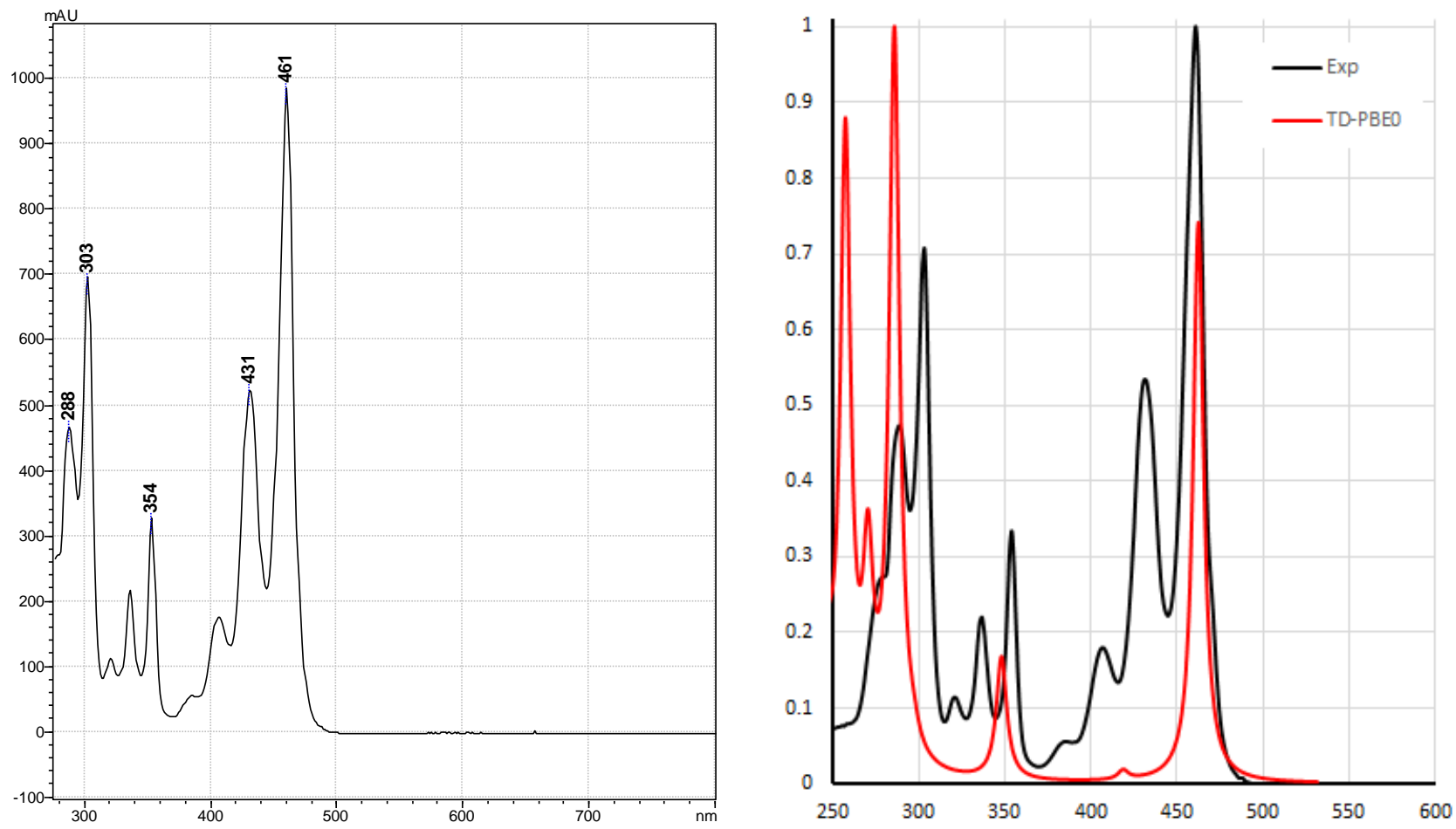

Figure S83. Left side: UV-Vis spectrum of NG5 (toluene-MeOH 4-1) from HPLC. Right side: Comparison of experimental (black) and predicted by TD-PBE0 UV/Vis spectra (red)

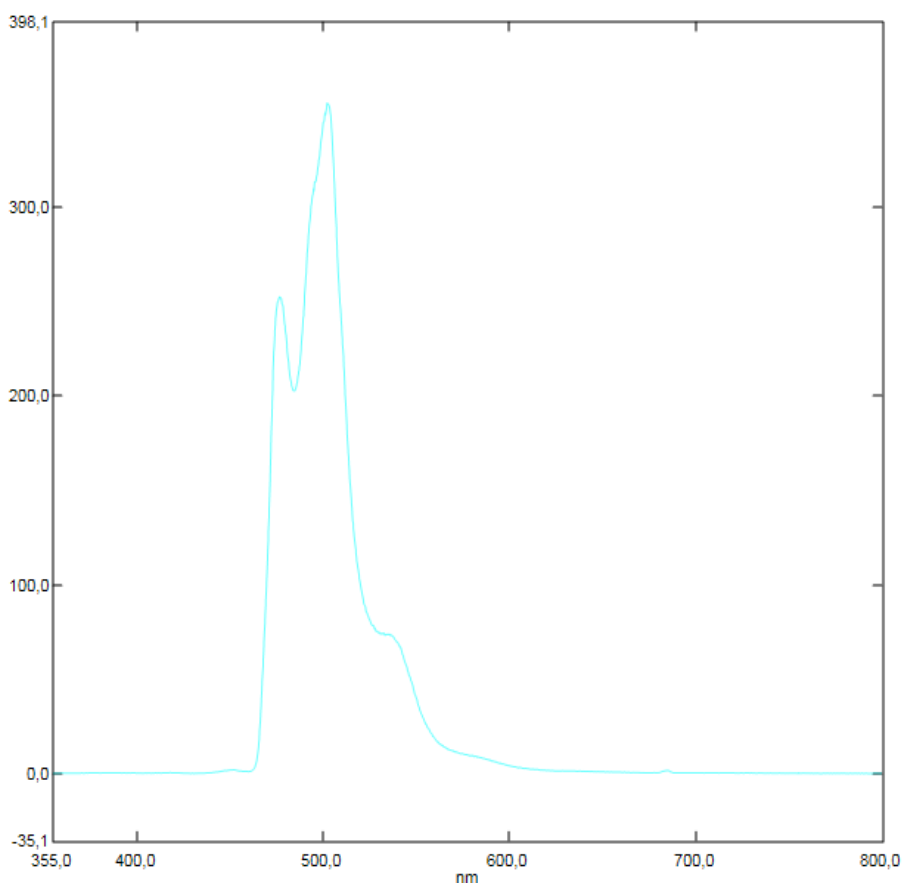

Figure S84. Fluorescence spectrum of NG5 (DCM). 


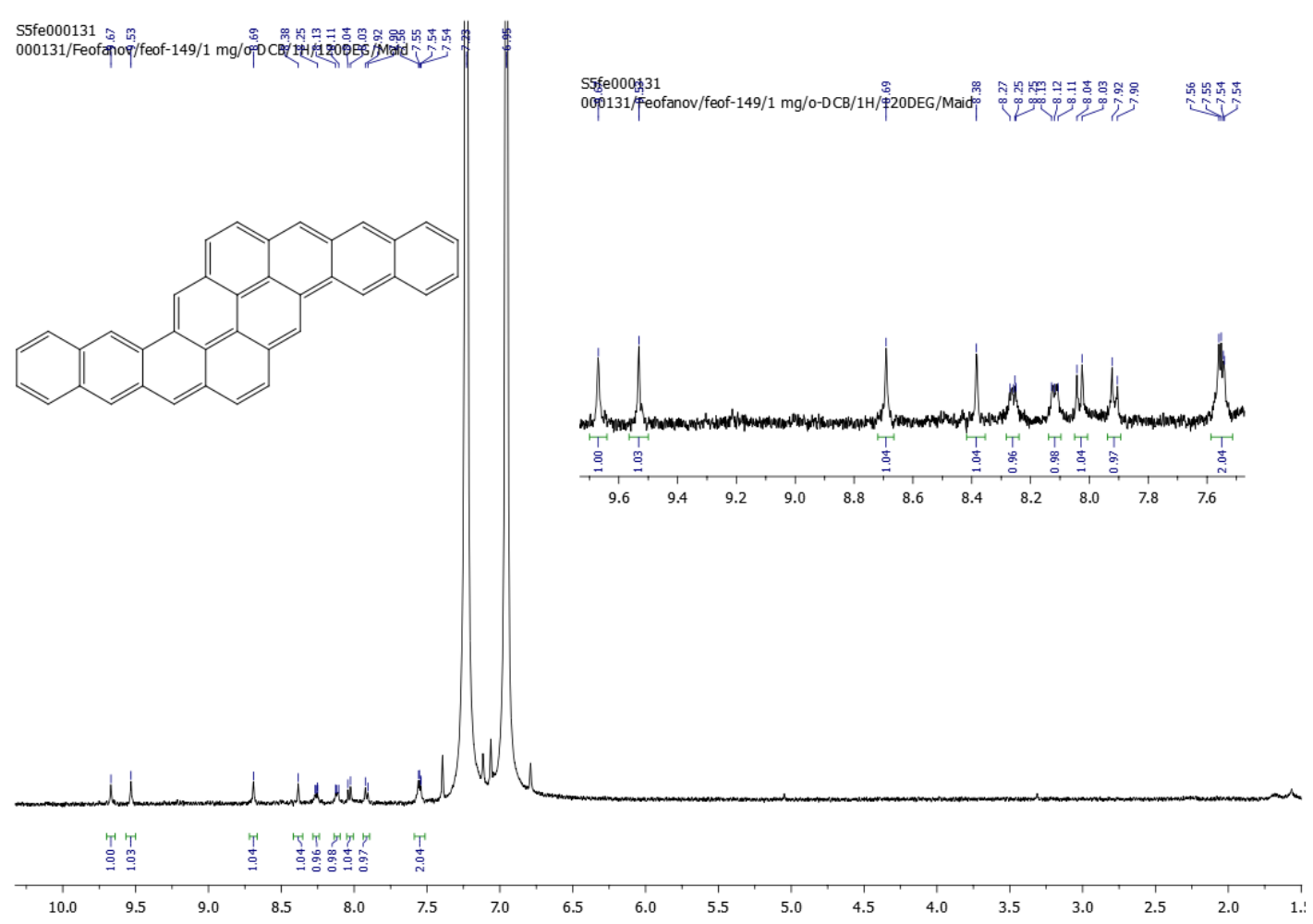

Figure S85. ${ }^{1} \mathrm{H}$ NMR (500 MHz, o- $\left.\mathrm{C}_{6} \mathrm{D}_{4} \mathrm{Cl}_{2}, 393 \mathrm{~K}\right)$ spectrum of NG6.

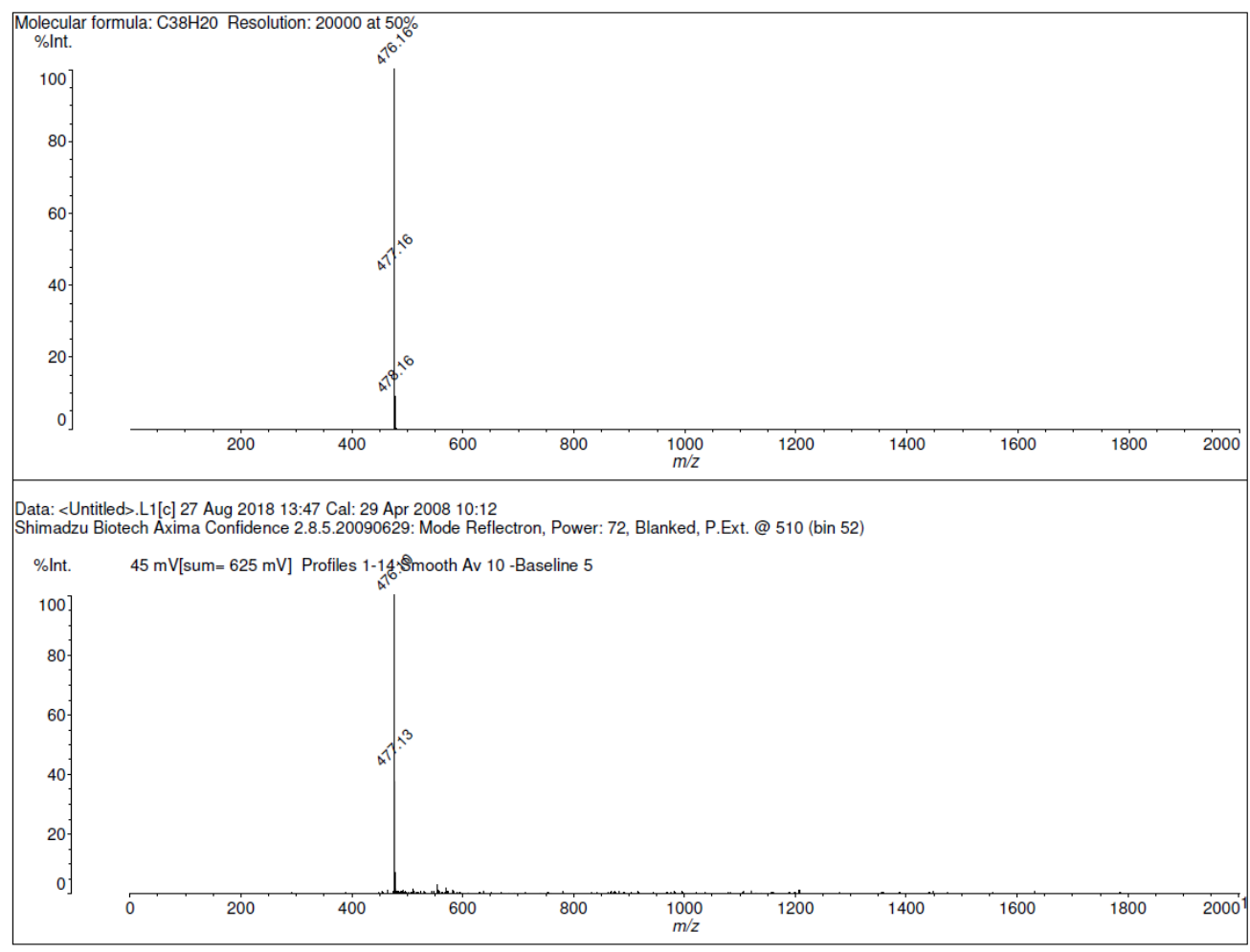

Figure S86. LDI-TOF MS spectrum of NG6. 


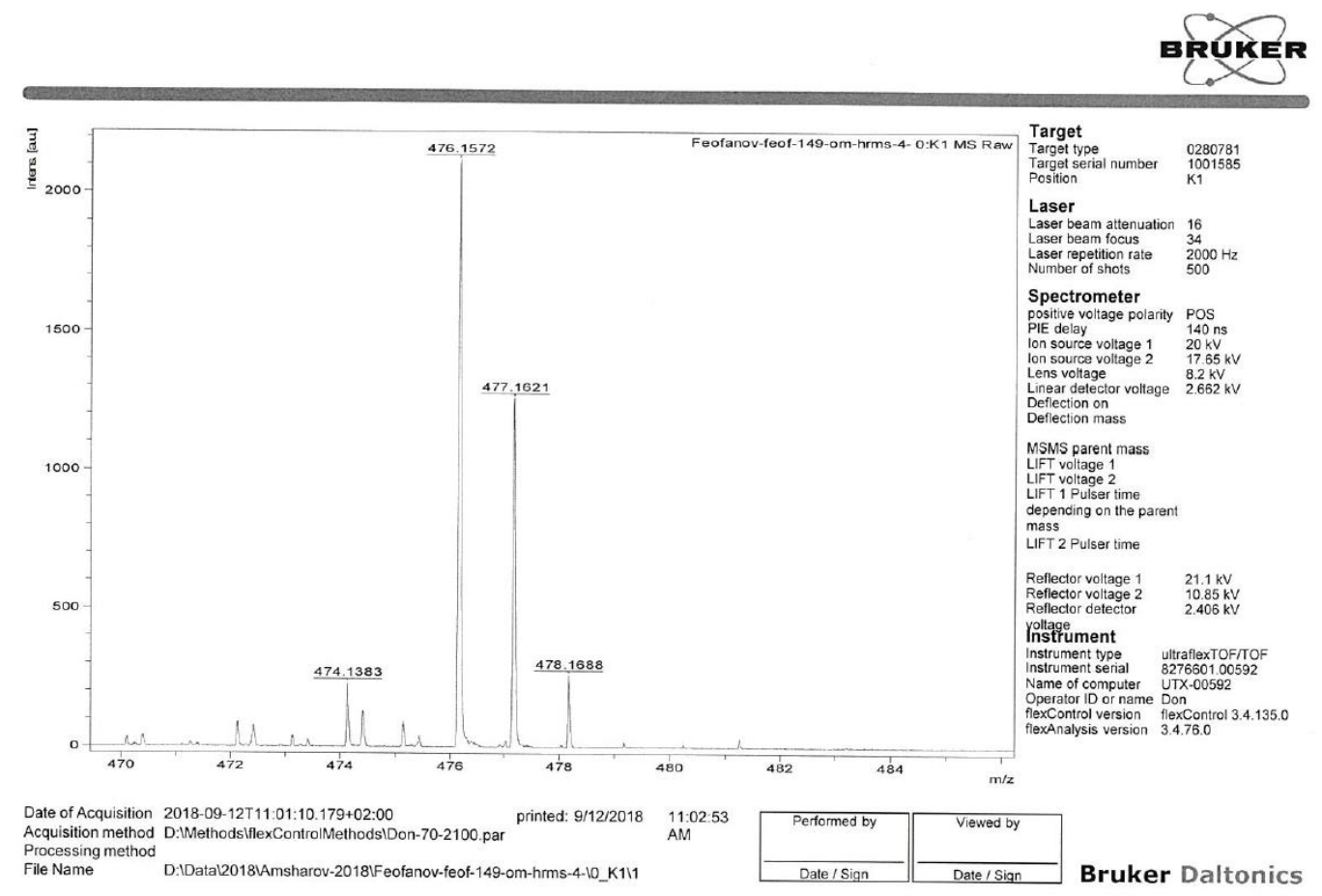

Figure S87. MALDI-TOF HRMS spectrum of NG6.

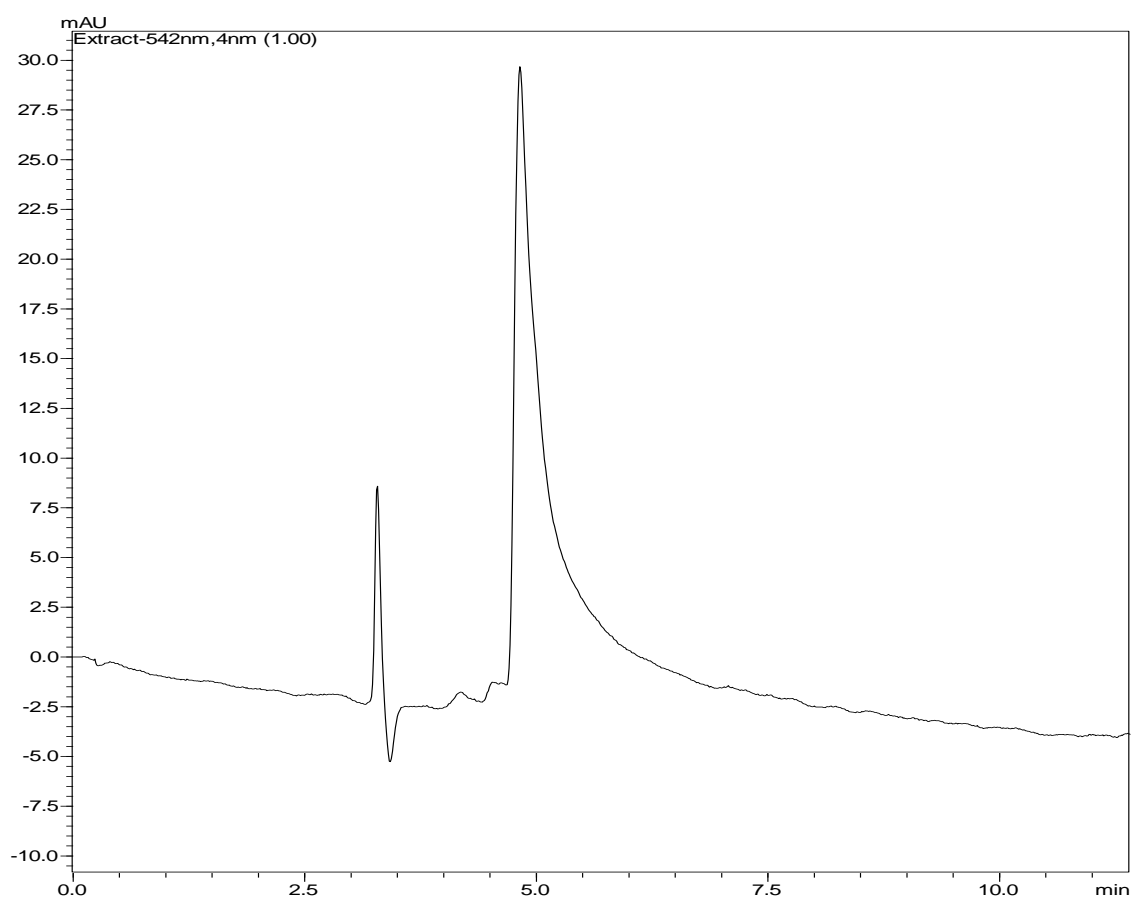

Figure S88. HPLC profile of NG6 after aqueous work-up; detected at 543 nm (PBr column, toluene-1.2dichlorobenzene $10-1$ as eluent, $1 \mathrm{~mL} / \mathrm{min}, 60^{\circ} \mathrm{C}$ ). 

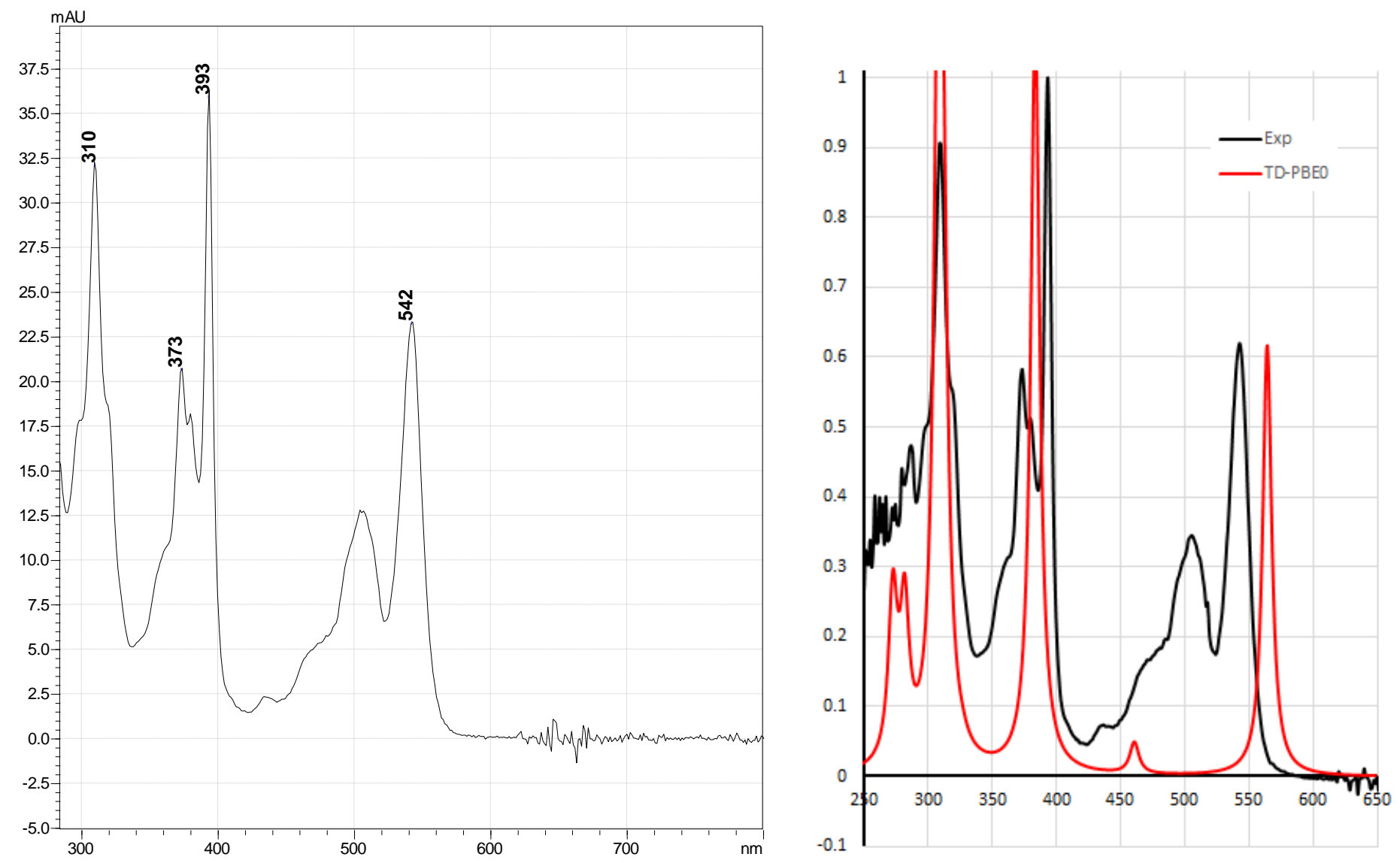

Figure S89. Left side: UV-Vis spectrum of NG6 (toluene-1.2-dichlorobenzene 10-1) from HPLC. Right side: Comparison of experimental (black) and predicted by TD-PBE0 UV/Vis spectra (red)

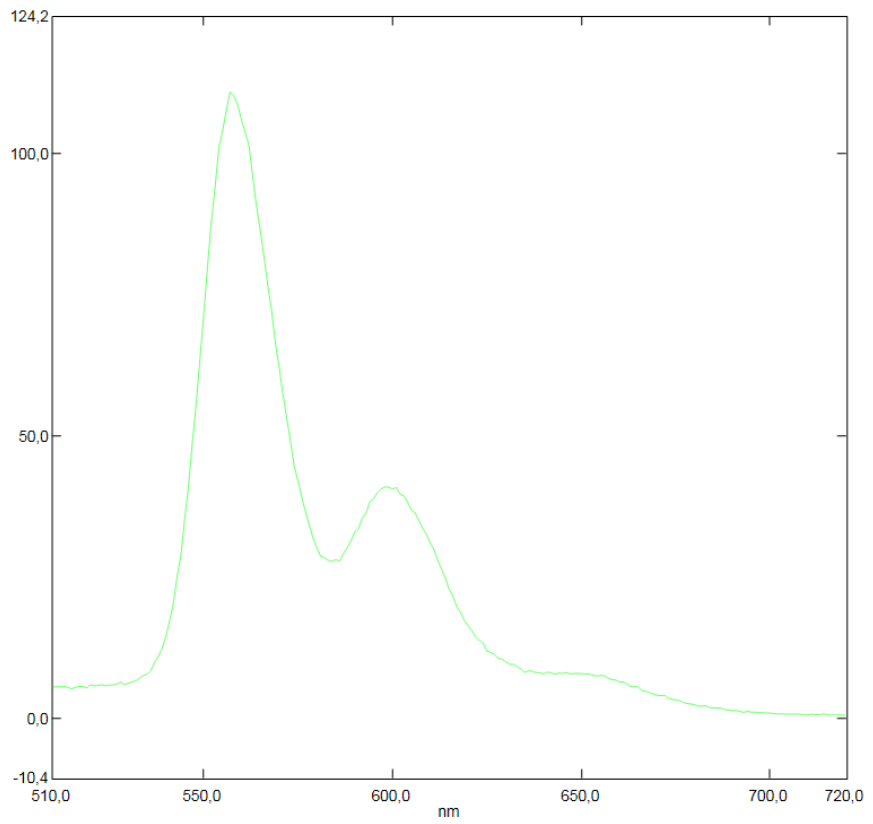

Figure S90. Fluorescence spectrum of NG6 (DCM). 


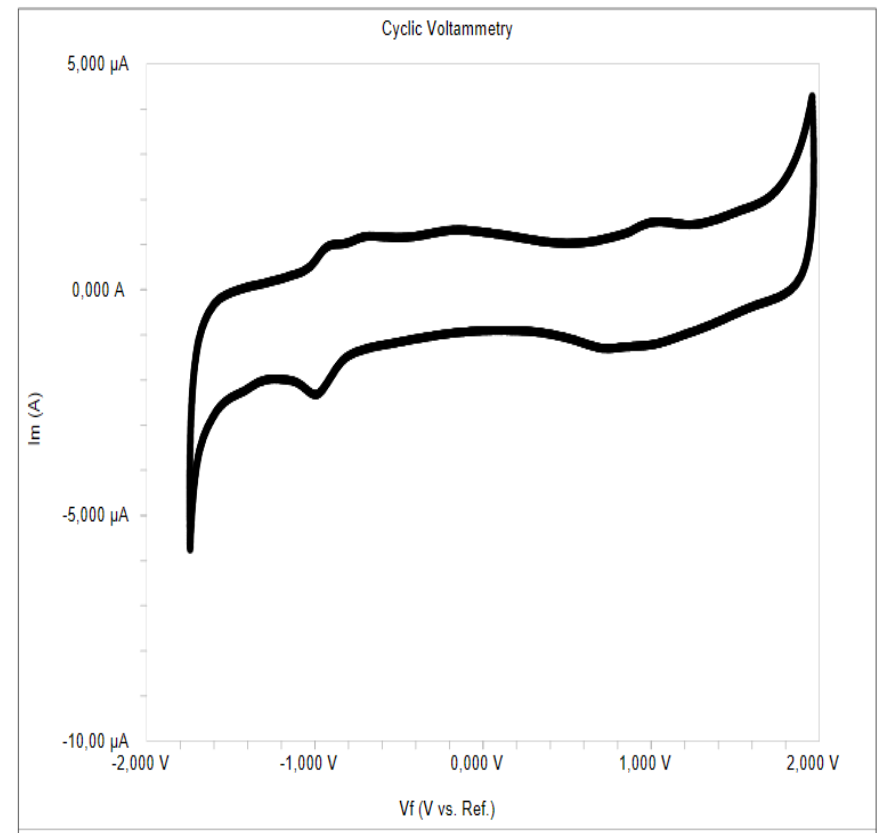

Figure S91. Cyclic voltammogram of NG6 measured in $0.1 \mathrm{M} \mathrm{Bu}_{4} \mathrm{NPF}_{6}$ in 1,2-dichlorobenzene at room temperature with $100 \mathrm{mV} / \mathrm{s}$ scan speed.

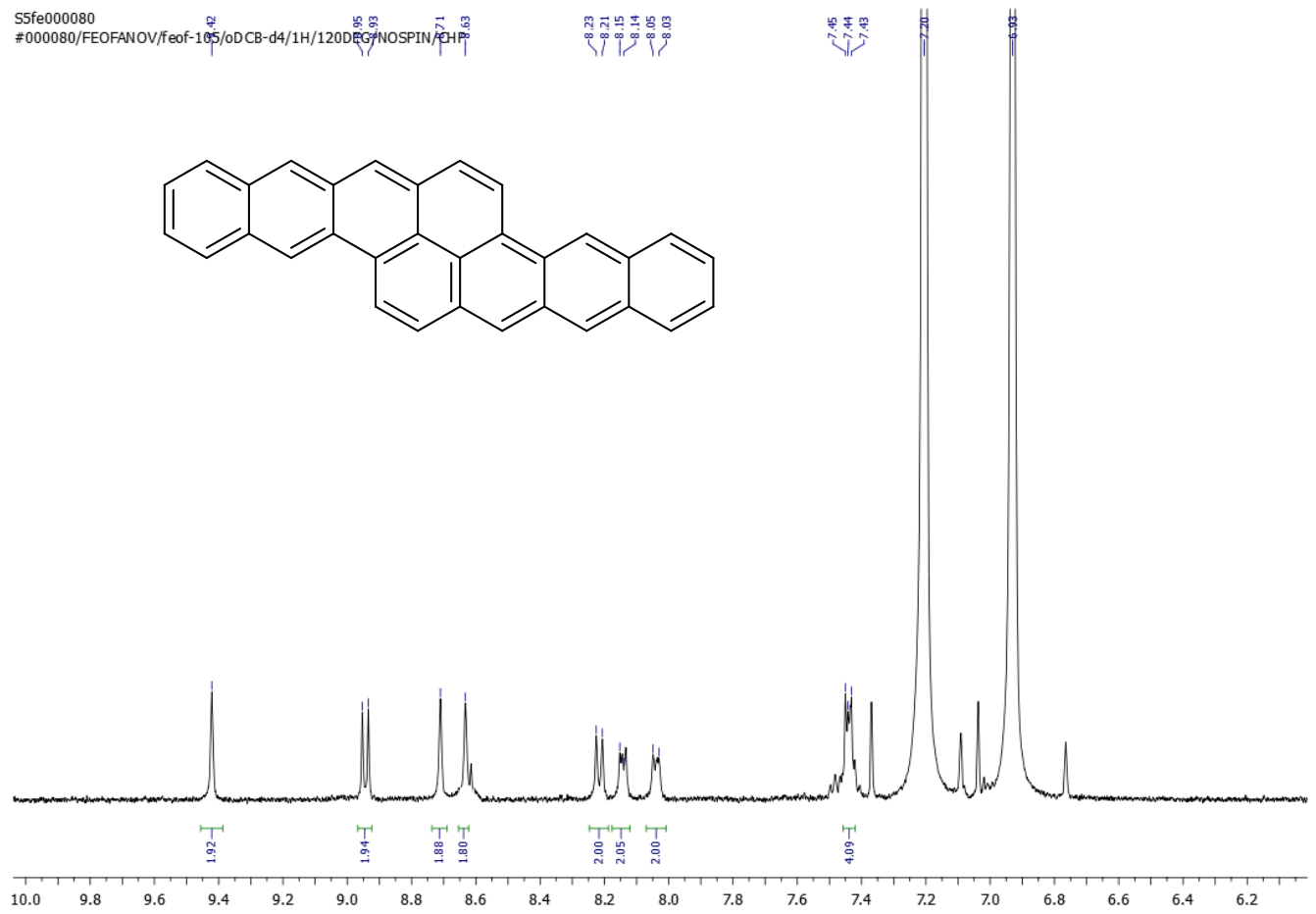

Figure S92. ${ }^{1} \mathrm{H}$ NMR (500 MHz, o- $\left.\mathrm{C}_{6} \mathrm{D}_{4} \mathrm{Cl}_{2}, 393 \mathrm{~K}\right)$ spectrum of NG7. 


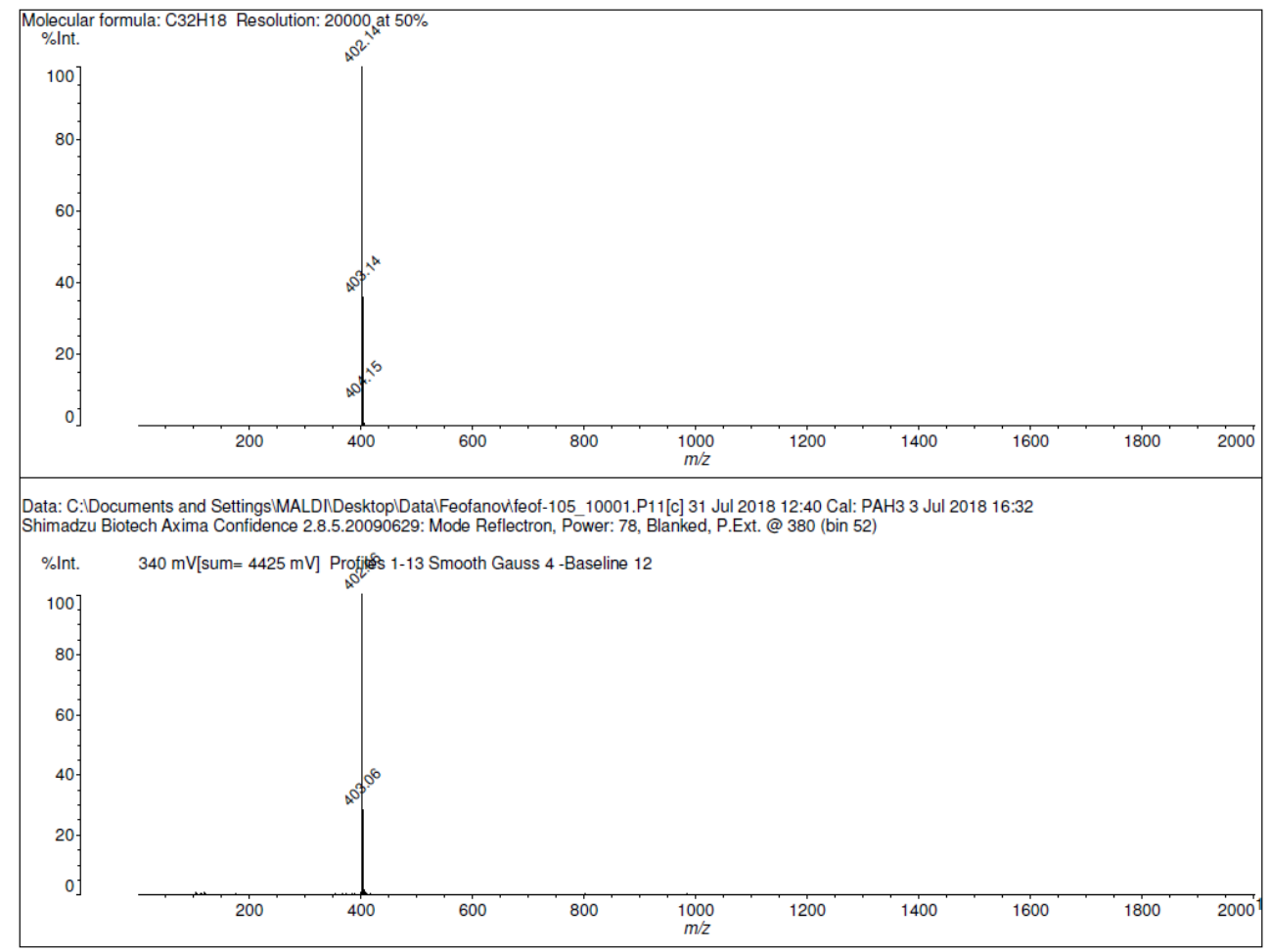

\section{Figure S93. LDI-TOF MS spectrum of NG7.}

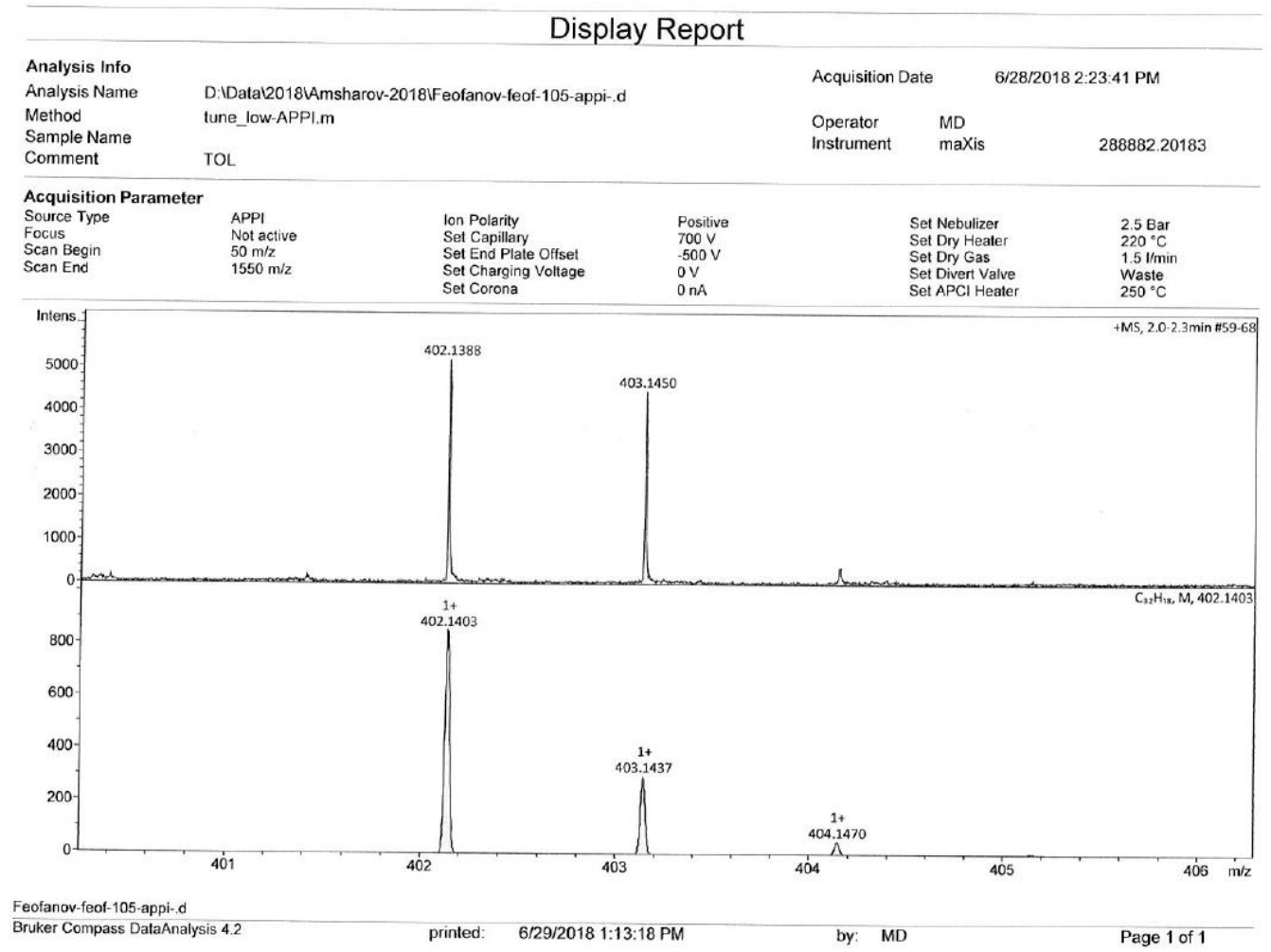

Figure S94. APPI-HRMS spectrum of NG7. 


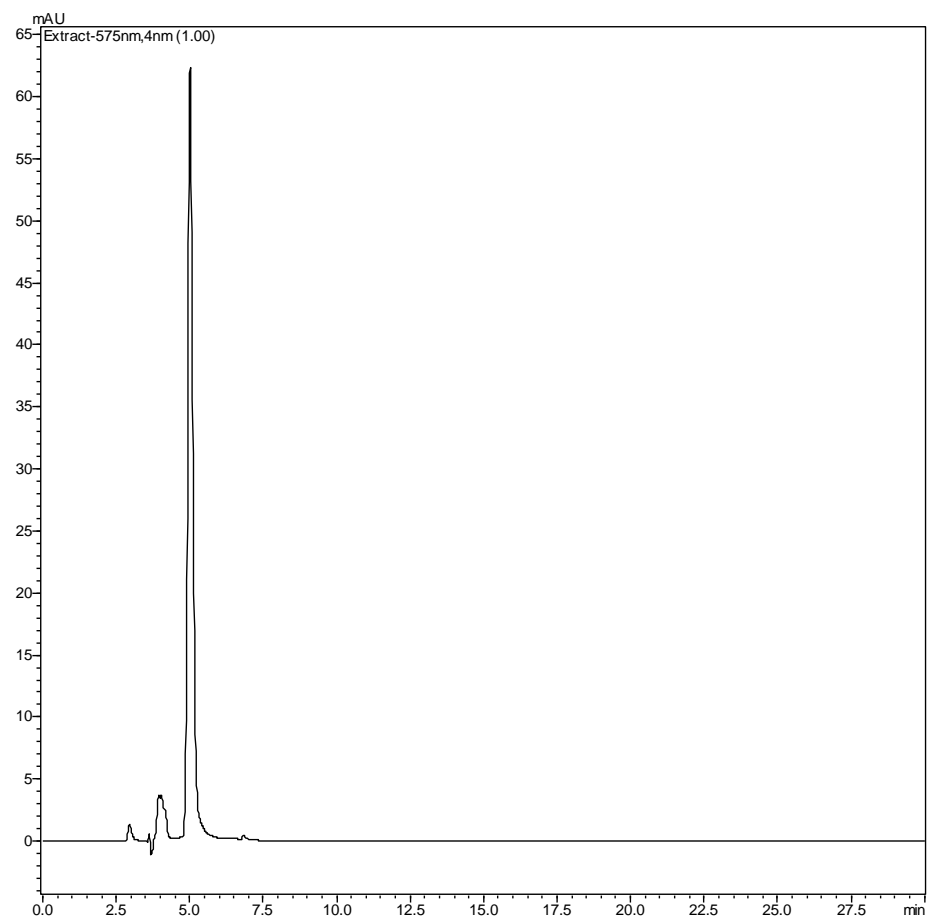

Figure S95. HPLC profile of NG7 after aqueous work-up; detected at $575 \mathrm{~nm}$ (PBr column, toluene, 1 $\left.\mathrm{mL} / \mathrm{min}, 40^{\circ} \mathrm{C}\right)$.
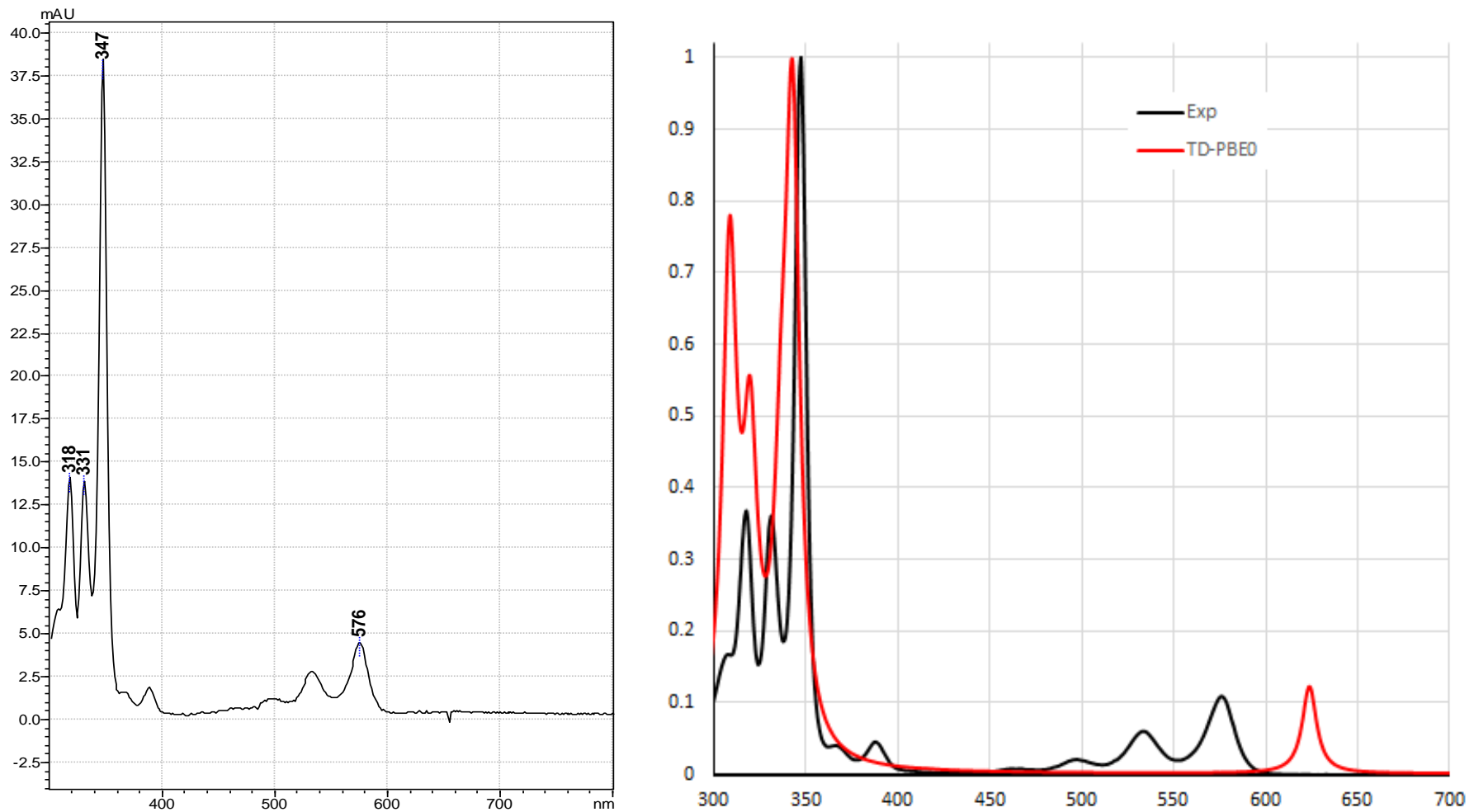

Figure S96. Left side: UV-Vis spectrum of NG7 (toluene) from HPLC. Right side: Comparison of experimental (black) and predicted by TD-PBE0 UV/Vis spectra (red) 


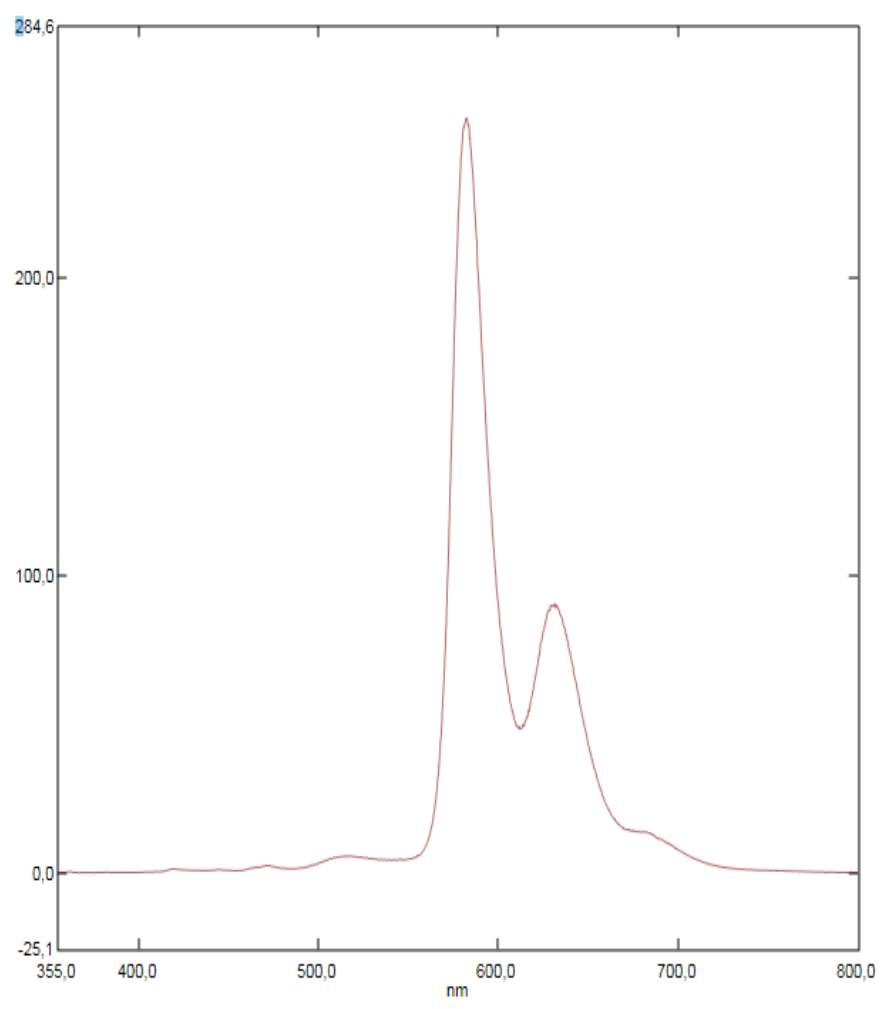

Figure S97. Fluorescence spectrum of NG7 (DCM).

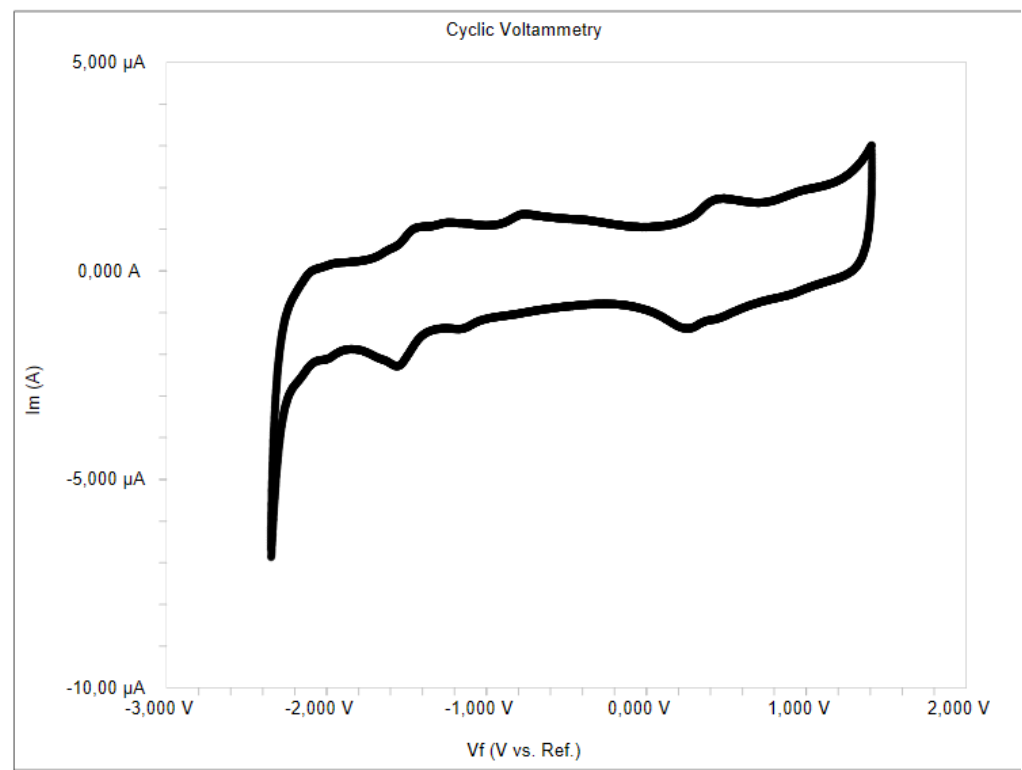

Figure S98. Cyclic voltammogram of NG7 measured in $0.1 \mathrm{M} \mathrm{Bu}_{4} \mathrm{NPF}_{6}$ in 1,2-dichlorobenzene at room temperature with $100 \mathrm{mV} / \mathrm{s}$ scan speed. 


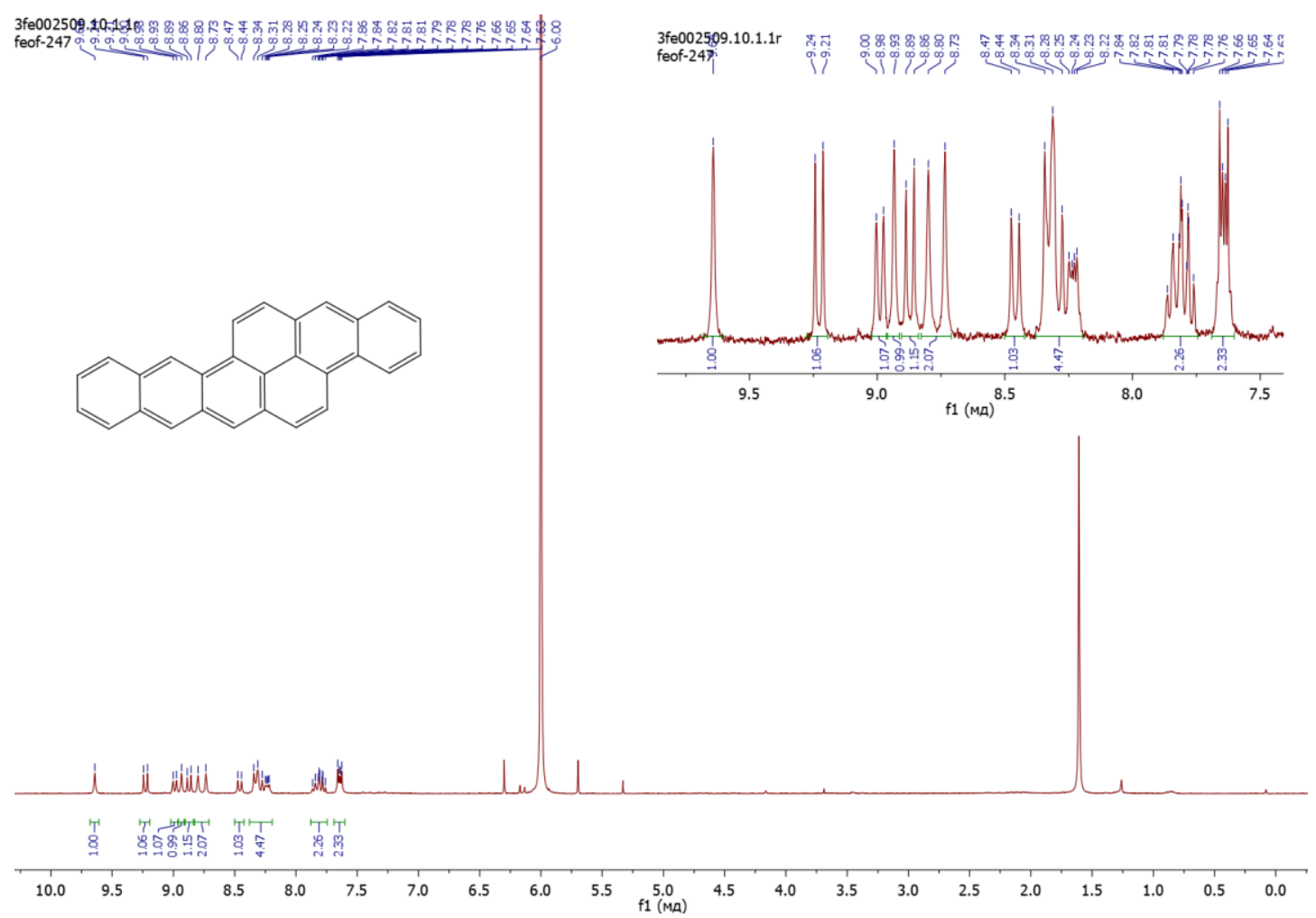

Figure S99. ${ }^{1} \mathrm{H}$ NMR (300 MHz, $\left.\mathrm{C}_{2} \mathrm{D}_{2} \mathrm{Cl}_{4}, 293 \mathrm{~K}\right)$ spectrum of NG8.

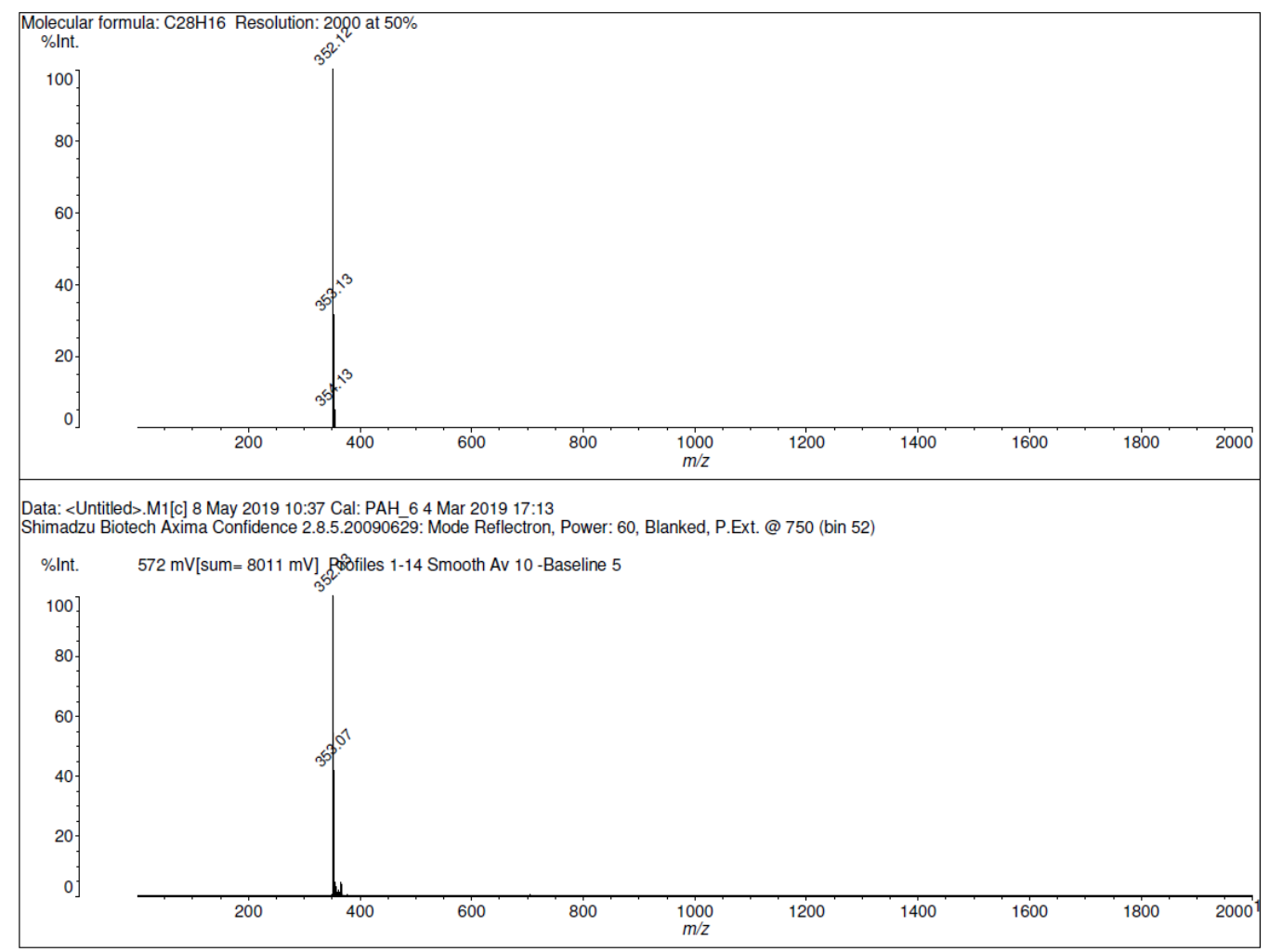

Figure S100. LDI-TOF MS spectrum of NG8. 


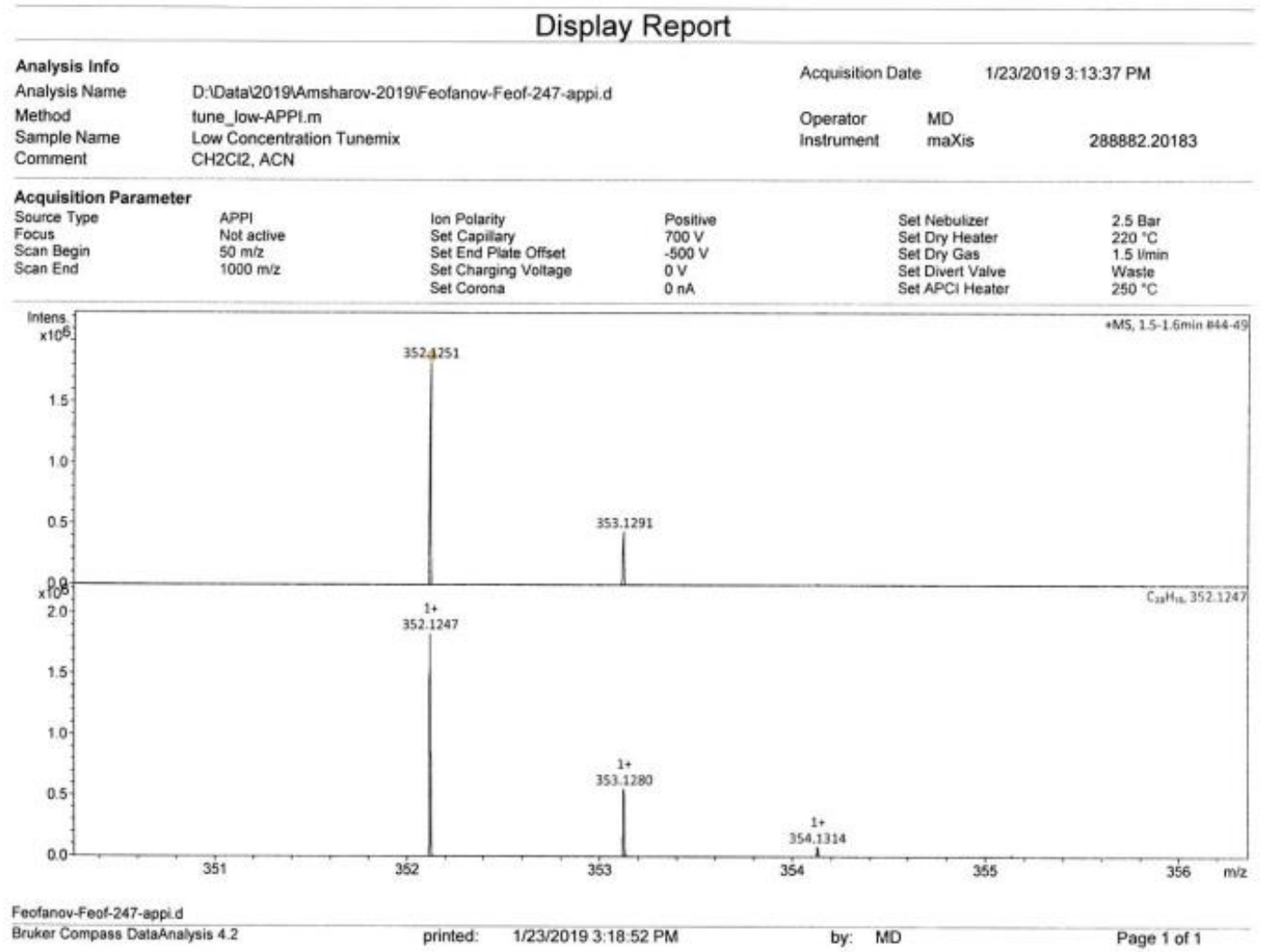

Figure S101. APPI-HRMS spectrum of NG8.

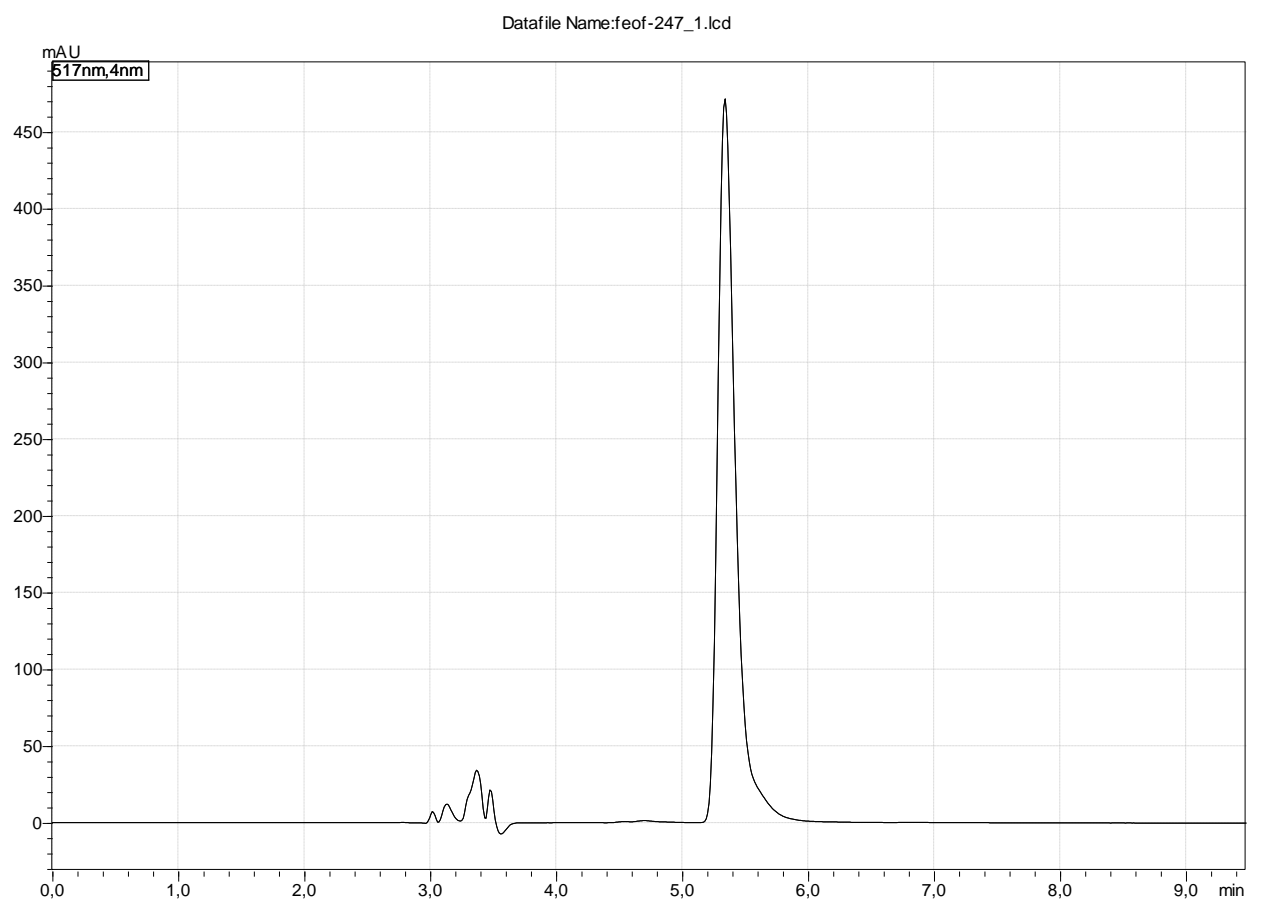

Figure S102. HPLC profile of NG8 after aqueous work-up; detected at $517 \mathrm{~nm}$ (PBr column, toluene, 1 $\mathrm{mL} / \mathrm{min}, 40^{\circ} \mathrm{C}$ ). 

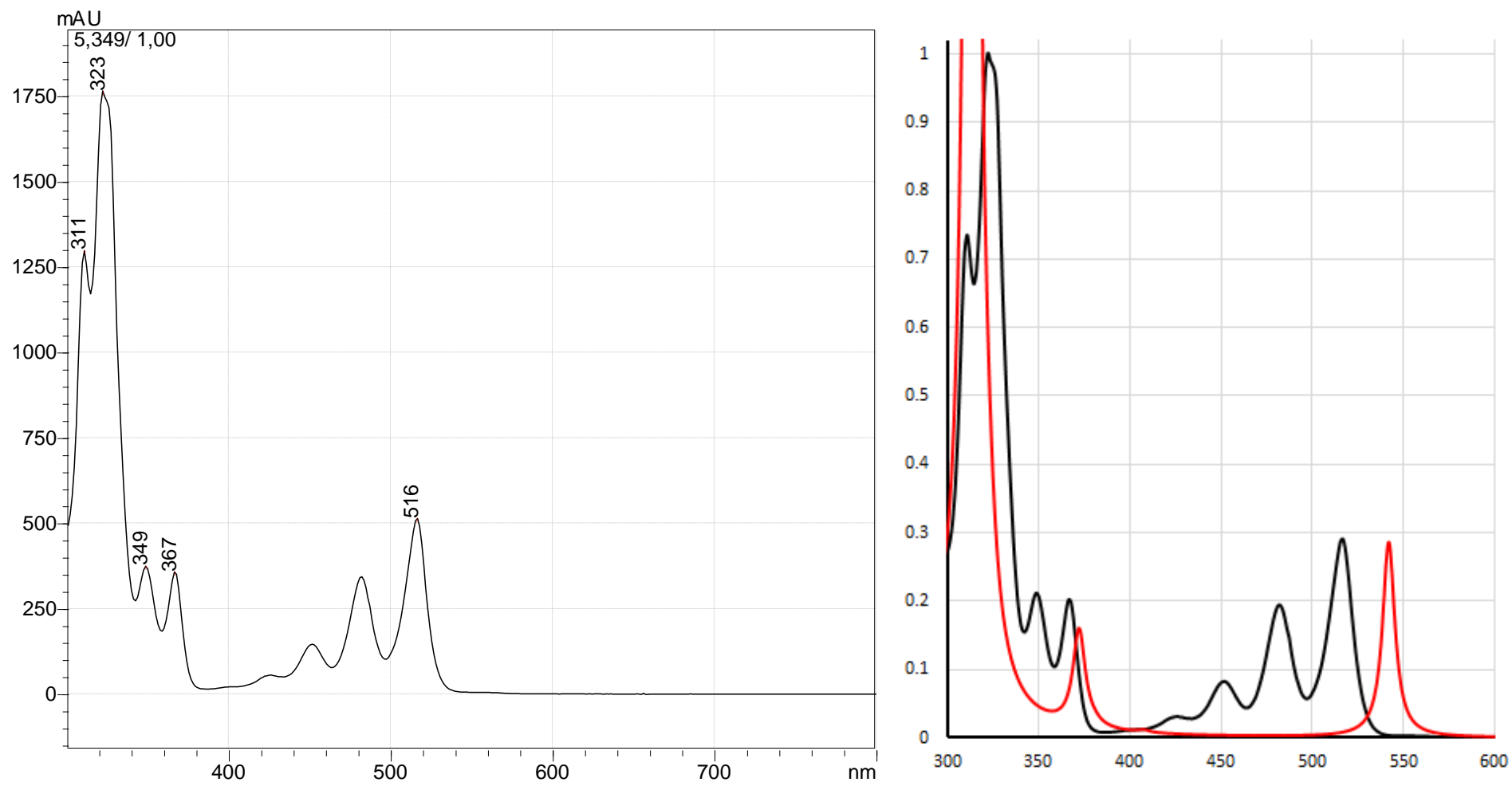

Figure S103. Left side: UV-Vis spectrum of NG8 (toluene) from HPLC. Right side: Comparison of experimental (black) and predicted by TD-PBE0 UV/Vis spectra (red)

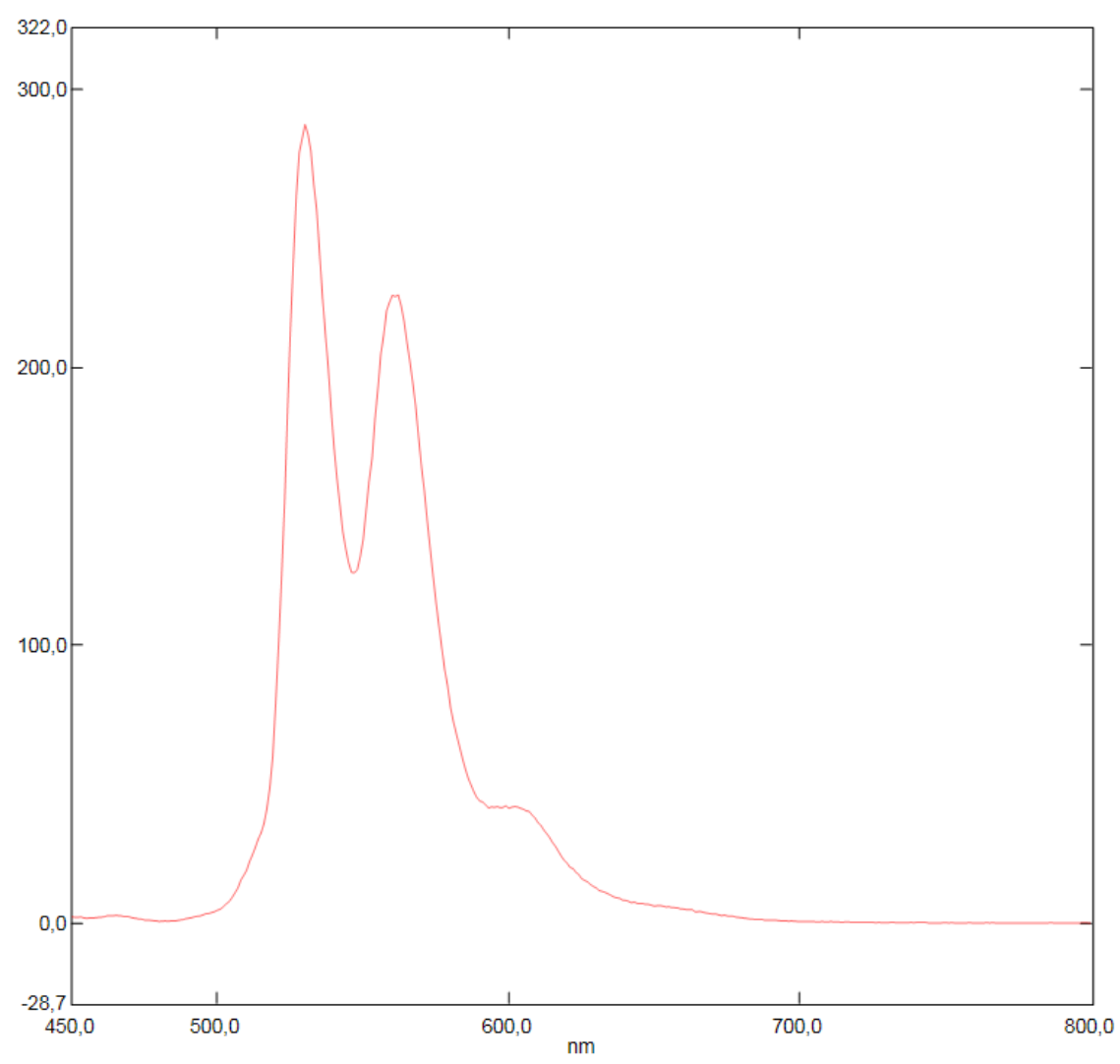


Figure S104. Fluorescence spectrum of NG8 (DCM).

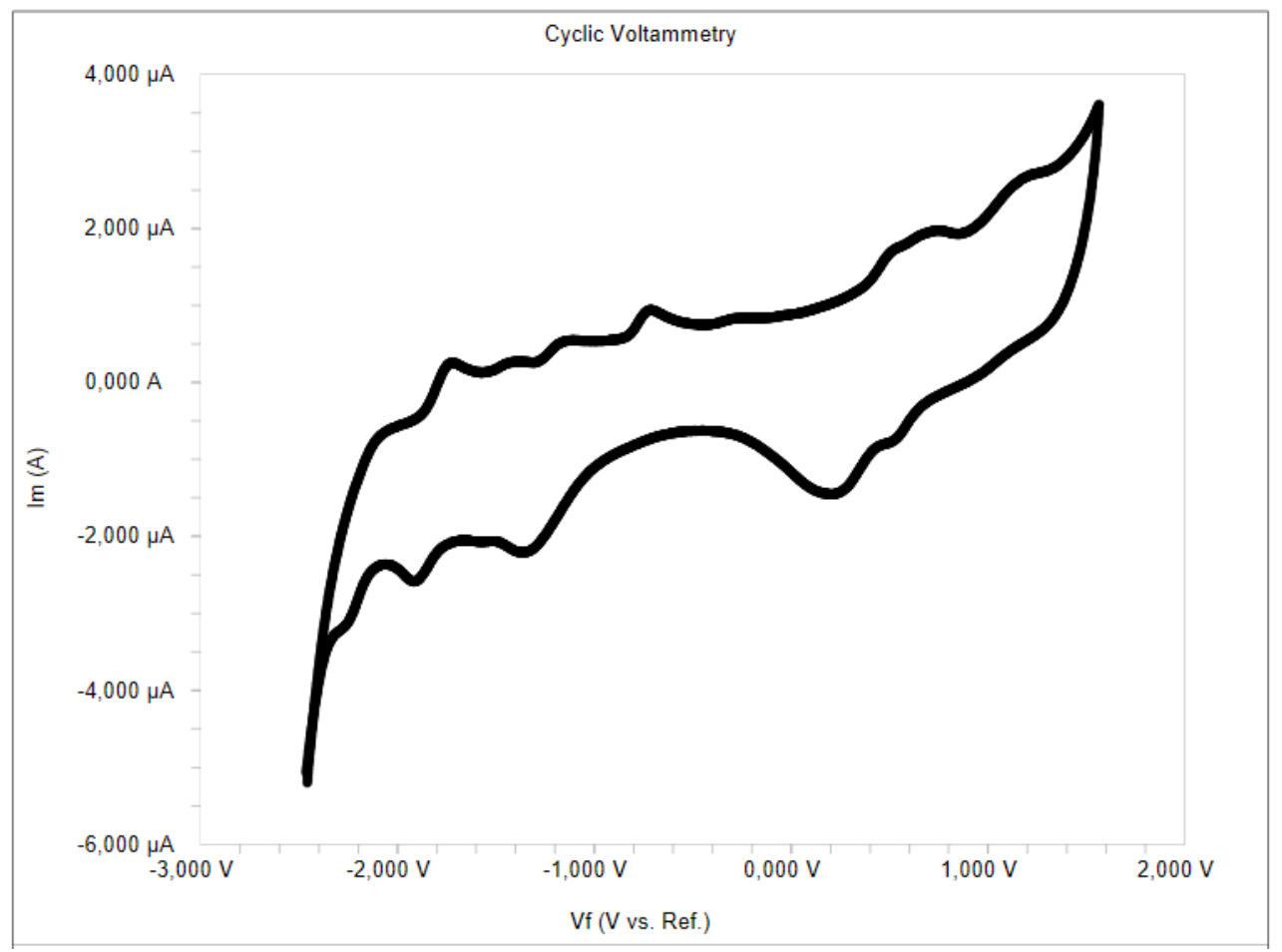

Figure S105. Cyclic voltammogram of NG8 measured in $0.1 \mathrm{M} \mathrm{Bu}_{4} \mathrm{NPF}_{6}$ in 1,2-dichlorobenzene at room temperature with $100 \mathrm{mV} / \mathrm{s}$ scan speed.

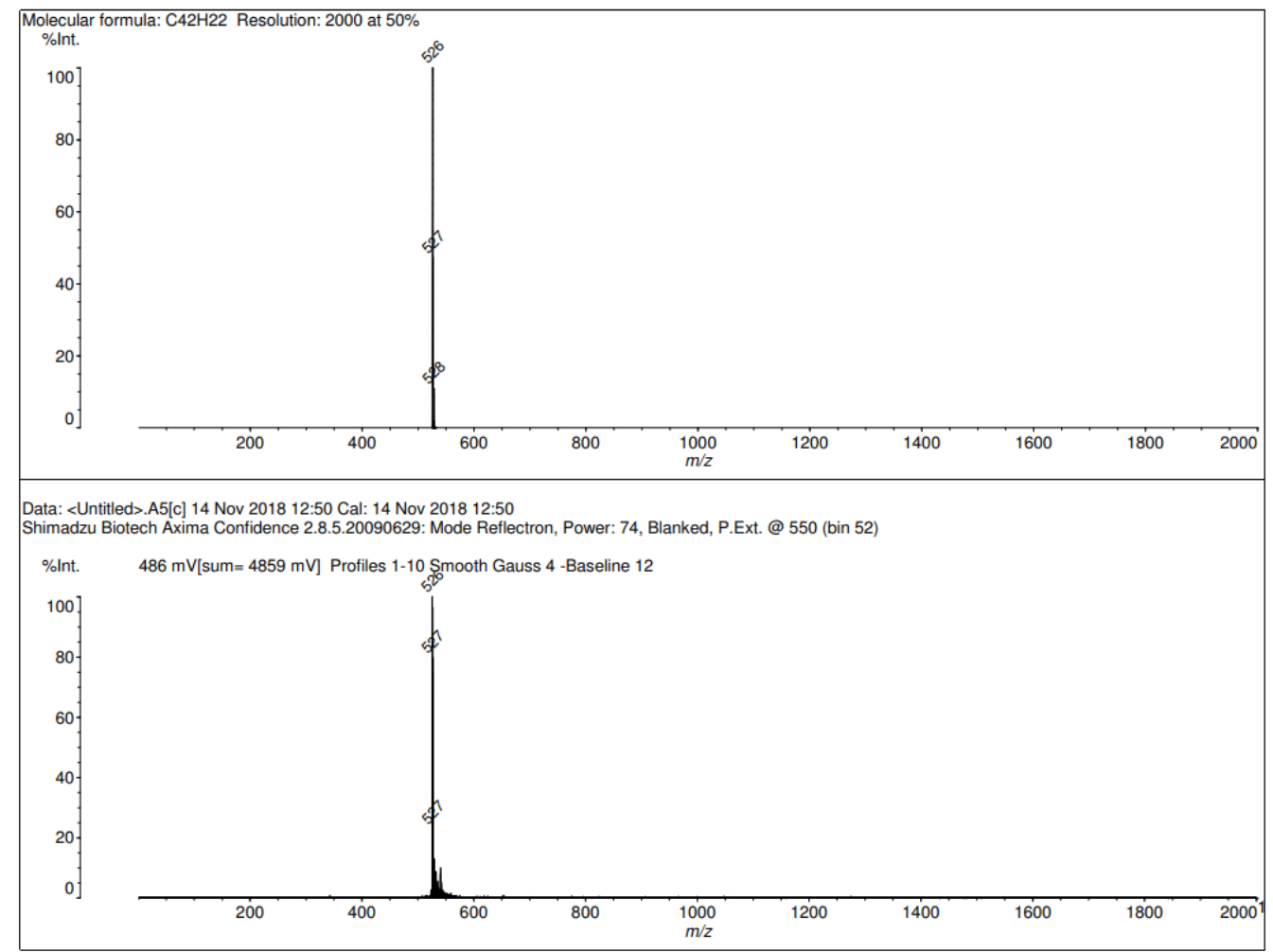


Figure S106. LDI-TOF MS spectrum of NG9.

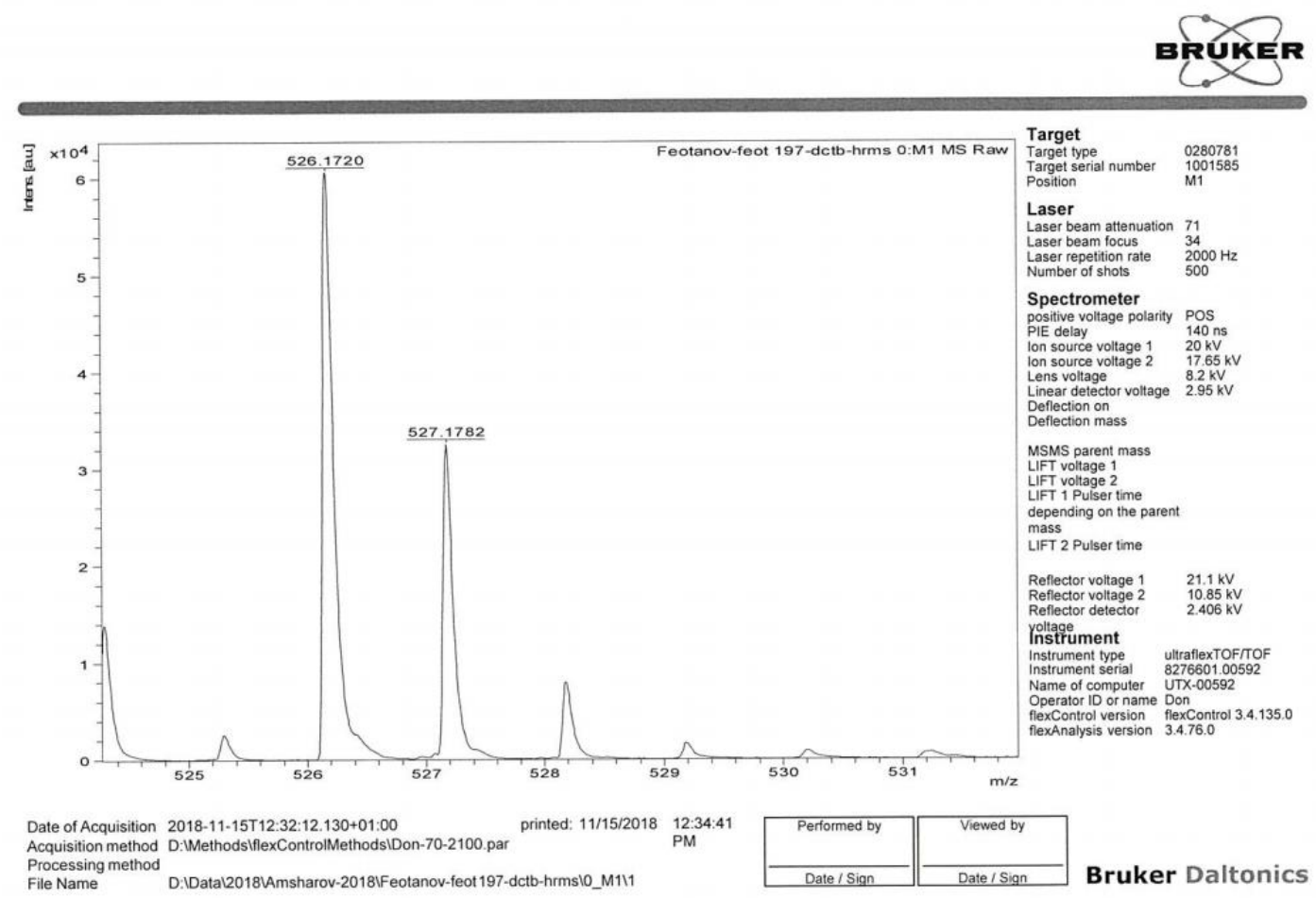

Figure S107. MALDI-TOF HRMS spectrum of NG9.

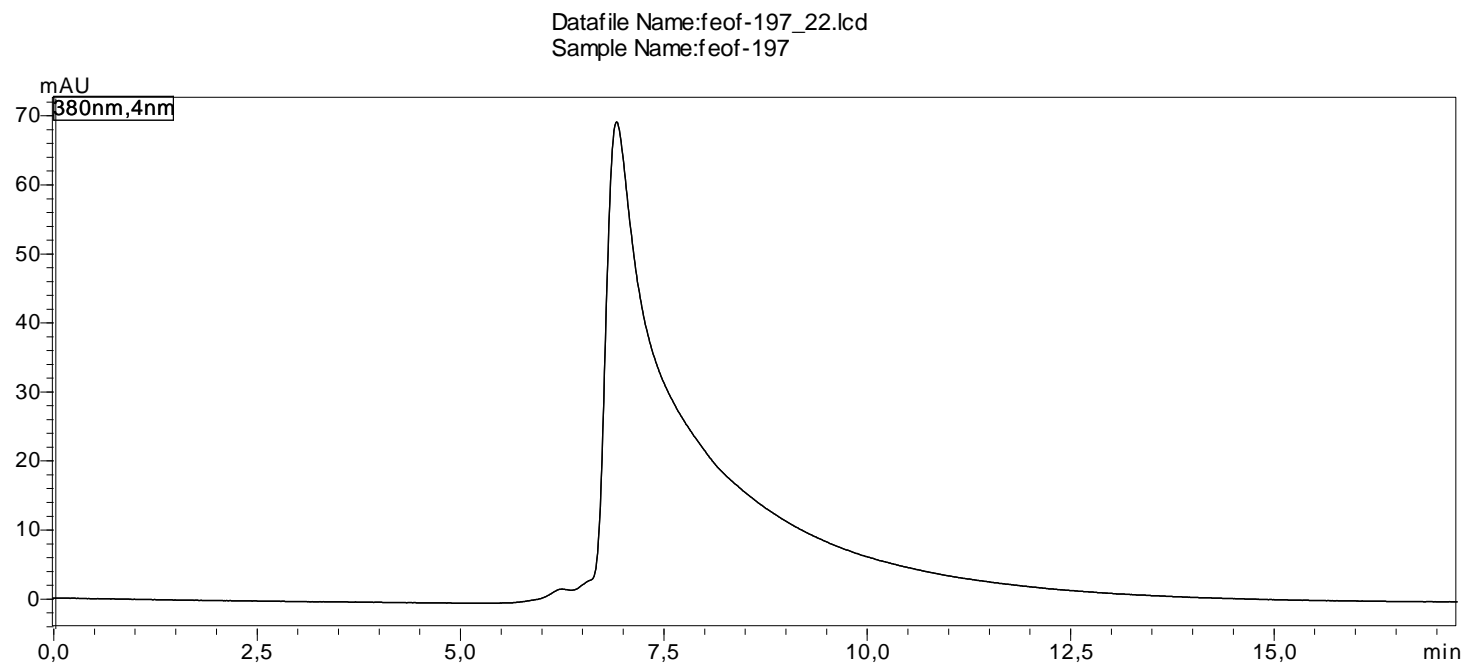

Figure S108. HPLC profile of NG9 after sublimation; detected at $380 \mathrm{~nm}(\mathrm{PBr}$ column, $o$-DCB, 0.5 $\left.\mathrm{mL} / \mathrm{min}, 85^{\circ} \mathrm{C}\right)$. 

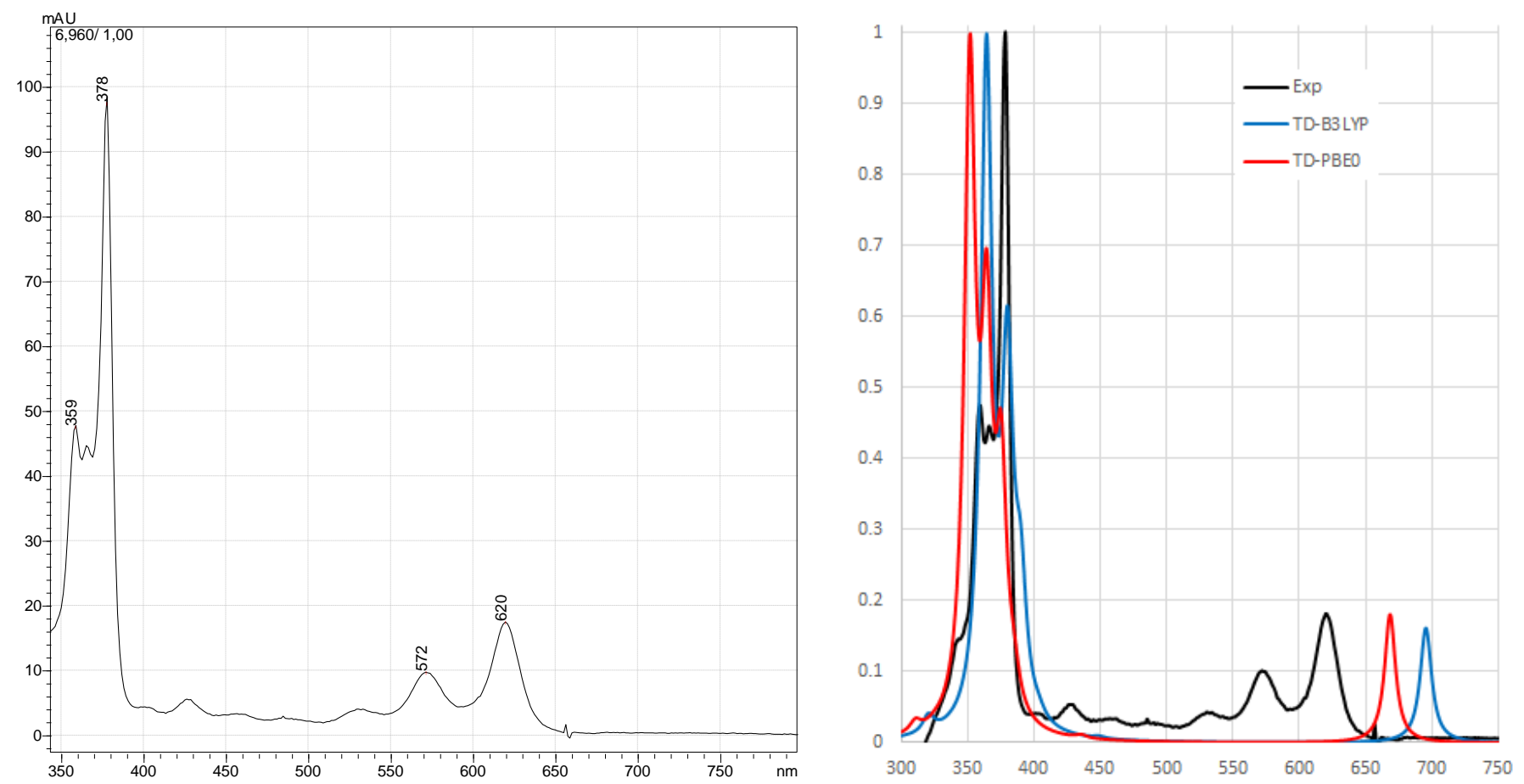

Figure S109. Left side: UV-Vis spectrum of NG9 (1,2-dichlorobenzene) from HPLC. Right side: Comparison of experimental (black) and predicted by TD-PBE0 (red) and TD-B3LYP (blue) $\mathrm{UV} / \mathrm{Vis}$ spectra.

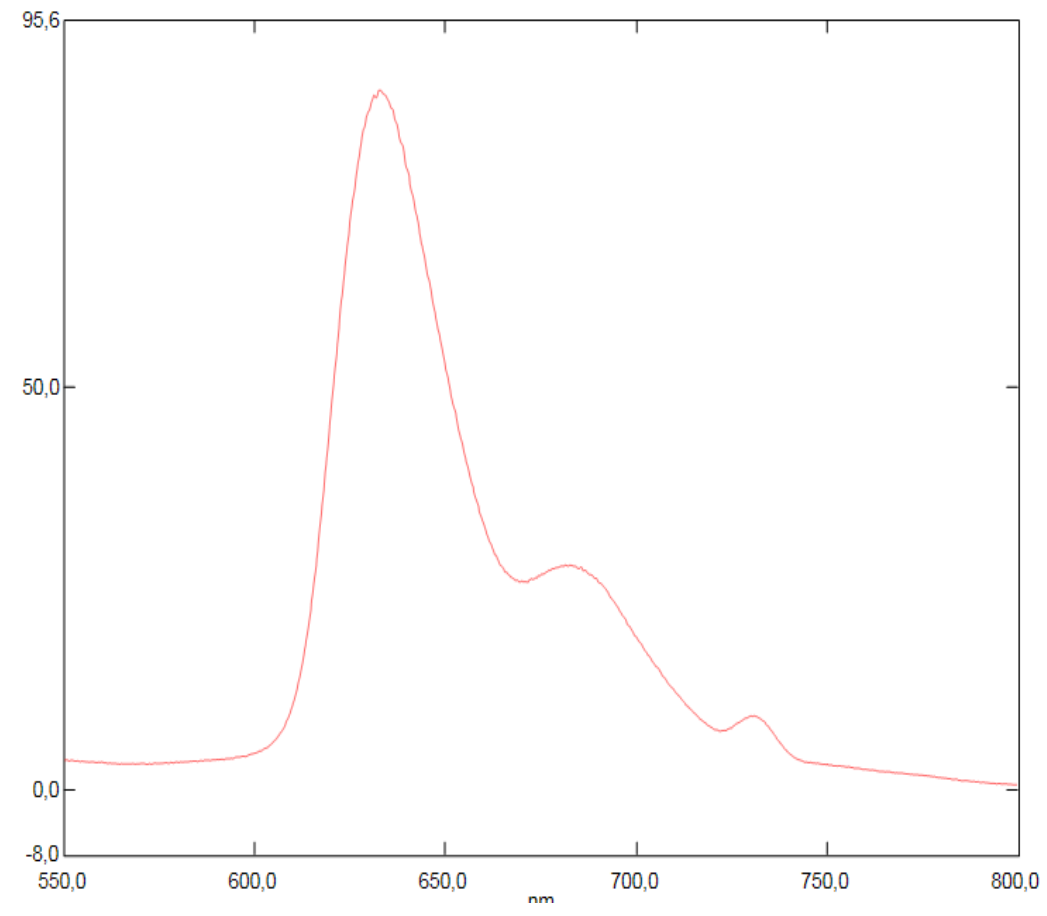

Figure S110. Fluorescence spectrum of NG9 (o-DCB). 


\section{Optimized geometry coordinates of NGs.}

\section{NG1}

44

symmetry c $2 \mathrm{v}$

$\begin{array}{lrrr}\mathrm{C} & 0.000000000 & 5.704308000 & -1.350436000 \\ \mathrm{C} & 0.000000000 & 4.981280000 & -0.181295000 \\ \mathrm{C} & 0.000000000 & 5.028098000 & -2.587683000 \\ \mathrm{H} & 0.000000000 & 5.488112000 & 0.778161000 \\ \mathrm{H} & 0.000000000 & 5.595335000 & -3.511588000 \\ \mathrm{C} & 0.000000000 & 3.562680000 & -0.200834000 \\ \mathrm{C} & 0.000000000 & 3.650385000 & -2.631840000 \\ \mathrm{H} & 0.000000000 & 3.165782000 & -3.599005000 \\ \mathrm{C} & 0.000000000 & 2.875207000 & -1.445986000 \\ \mathrm{H} & 0.000000000 & 6.788047000 & -1.324987000 \\ \mathrm{C} & 0.000000000 & 1.429539000 & -1.433428000 \\ \mathrm{C} & 0.000000000 & 0.717868000 & -0.203921000 \\ \mathrm{C} & 0.000000000 & 0.682338000 & -2.639564000 \\ \mathrm{C} & 0.000000000 & -0.717868000 & -0.203921000 \\ \mathrm{C} & 0.000000000 & -0.682338000 & -2.639564000 \\ \mathrm{C} & 0.000000000 & -1.429539000 & -1.433428000 \\ \mathrm{H} & 0.000000000 & 1.196983000 & -3.590546000 \\ \mathrm{H} & 0.000000000 & -1.196983000 & -3.590546000 \\ \mathrm{C} & 0.000000000 & -2.875207000 & -1.445986000 \\ \mathrm{C} & 0.000000000 & -3.562680000 & -0.200834000 \\ \mathrm{C} & 0.000000000 & -3.650385000 & -2.631840000 \\ \mathrm{C} & 0.000000000 & -4.981280000 & -0.181295000 \\ \mathrm{C} & 0.000000000 & -5.028098000 & -2.587683000 \\ \mathrm{C} & 0.000000000 & -5.704308000 & -1.350436000 \\ \mathrm{H} & 0.000000000 & -3.165782000 & -3.599005000 \\ \mathrm{H} & 0.000000000 & -5.488112000 & 0.778161000 \\ \mathrm{H} & 0.000000000 & -5.595335000 & -3.511588000 \\ \mathrm{H} & 0.000000000 & -6.788047000 & -1.324987000 \\ \mathrm{C} & 0.000000000 & 2.820710000 & 1.006289000 \\ \mathrm{H} & 0.000000000 & 3.393689000 & 1.923767000 \\ \mathrm{C} & 0.000000000 & 1.444791000 & 1.043532000 \\ \mathrm{C} & 0.000000000 & -1.444791000 & 1.043532000 \\ \mathrm{C} & 0.000000000 & -2.820710000 & 1.006289000 \\ \mathrm{H} & 0.000000000 & -3.393689000 & 1.923767000 \\ \mathrm{C} & 0.000000000 & -0.706702000 & 2.317708000 \\ \mathrm{C} & 0.000000000 & 0.706702000 & 2.317708000 \\ \mathrm{C} & 0.000000000 & -1.380411000 & 3.556399000 \\ \mathrm{C} & 0.000000000 & 1.380411000 & 3.556399000 \\ \mathrm{C} & 0.000000000 & -0.698943000 & 4.758888000 \\ \mathrm{H} & 0.000000000 & 0.698943000 & 4.758888000 \\ & 0.000000000 & -2.461147000 & 3.584161000 \\ \mathrm{H} & 0.000000000 & 2.461147000 & 3.584161000 \\ & 0.000000000 & -1.248813000 & 5.693081000 \\ & & \end{array}$

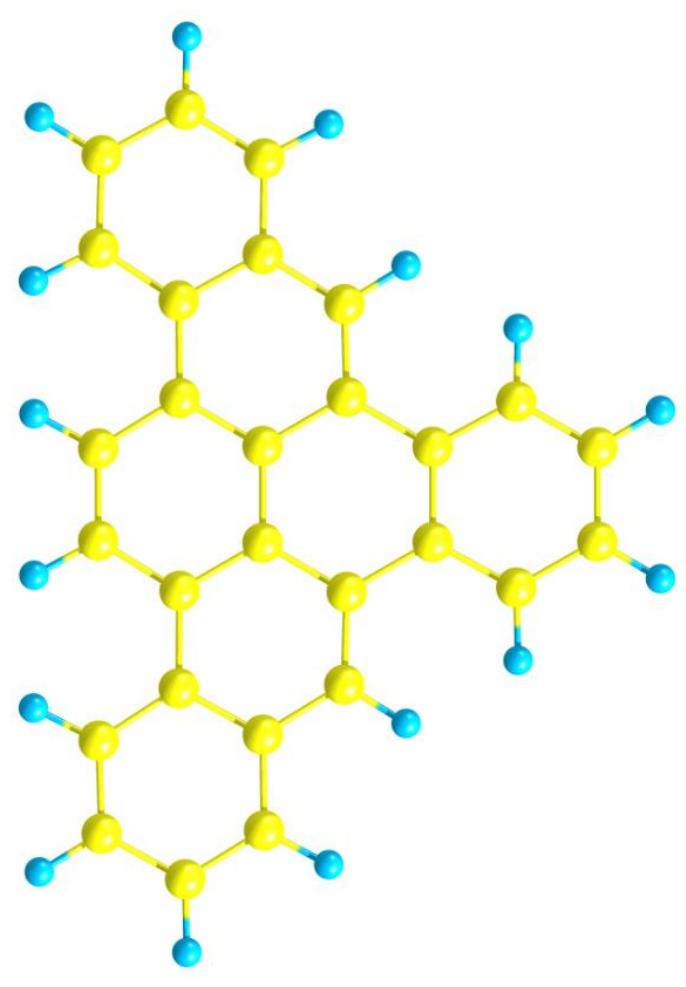




\section{NG2}

44

symmetry c $2 \mathrm{~h}$

$\begin{array}{lrrr}\mathrm{C} & 2.359608000 & 5.731618000 & 0.000000000 \\ \mathrm{C} & 1.198201000 & 4.991648000 & 0.000000000 \\ \mathrm{C} & 3.602184000 & 5.071924000 & 0.000000000 \\ \mathrm{H} & 0.232463000 & 5.486512000 & 0.000000000 \\ \mathrm{H} & 4.519998000 & 5.648944000 & 0.000000000 \\ \mathrm{C} & 1.236564000 & 3.576033000 & 0.000000000 \\ \mathrm{C} & 3.661277000 & 3.691987000 & 0.000000000 \\ \mathrm{H} & 4.634116000 & 3.218578000 & 0.000000000 \\ \mathrm{C} & 2.487360000 & 2.905536000 & 0.000000000 \\ \mathrm{H} & 2.319478000 & 6.814944000 & 0.000000000 \\ \mathrm{C} & 2.493458000 & 1.450399000 & 0.000000000 \\ \mathrm{C} & 1.259002000 & 0.728682000 & 0.000000000 \\ \mathrm{C} & 3.701392000 & 0.726537000 & 0.000000000 \\ \mathrm{C} & 1.269897000 & -0.696903000 & 0.000000000 \\ \mathrm{C} & 3.701392000 & -0.652938000 & 0.000000000 \\ \mathrm{C} & 2.497822000 & -1.356420000 & 0.000000000 \\ \mathrm{H} & 4.647803000 & 1.249361000 & 0.000000000 \\ \mathrm{H} & 4.639841000 & -1.195861000 & 0.000000000 \\ \mathrm{C} & -2.487360000 & -2.905536000 & 0.000000000 \\ \mathrm{C} & -1.236564000 & -3.576033000 & 0.000000000 \\ \mathrm{C} & -3.661277000 & -3.691987000 & 0.000000000 \\ \mathrm{C} & -1.198201000 & -4.991648000 & 0.000000000 \\ \mathrm{C} & -3.602184000 & -5.071924000 & 0.000000000 \\ \mathrm{C} & -2.359608000 & -5.731618000 & 0.000000000 \\ \mathrm{H} & -4.634116000 & -3.218578000 & 0.000000000 \\ \mathrm{H} & -0.232463000 & -5.486512000 & 0.000000000 \\ \mathrm{H} & -4.519998000 & -5.648944000 & 0.000000000 \\ \mathrm{H} & -2.319478000 & -6.814944000 & 0.000000000 \\ \mathrm{C} & 0.031702000 & 2.818869000 & 0.000000000 \\ \mathrm{H} & -0.889146000 & 3.386889000 & 0.000000000 \\ \mathrm{C} & 0.004043000 & 1.447519000 & 0.000000000 \\ \mathrm{C} & -0.004043000 & -1.447519000 & 0.000000000 \\ \mathrm{C} & -0.031702000 & -2.818869000 & 0.000000000 \\ \mathrm{H} & 0.889146000 & -3.386889000 & 0.000000000 \\ \mathrm{C} & -1.259002000 & -0.728682000 & 0.000000000 \\ \mathrm{C} & -1.269897000 & 0.696903000 & 0.000000000 \\ \mathrm{C} & -2.493458000 & -1.450399000 & 0.000000000 \\ \mathrm{C} & -2.497822000 & 1.356420000 & 0.000000000 \\ \mathrm{C} & -3.701392000 & -0.726537000 & 0.000000000 \\ \mathrm{C} & -3.701392000 & 0.652938000 & 0.000000000 \\ \mathrm{H} & 2.540826000 & -2.436575000 & 0.000000000 \\ & -2.540826000 & 2.436575000 & 0.000000000 \\ & -4.639841000 & 1.195861000 & 0.000000000\end{array}$

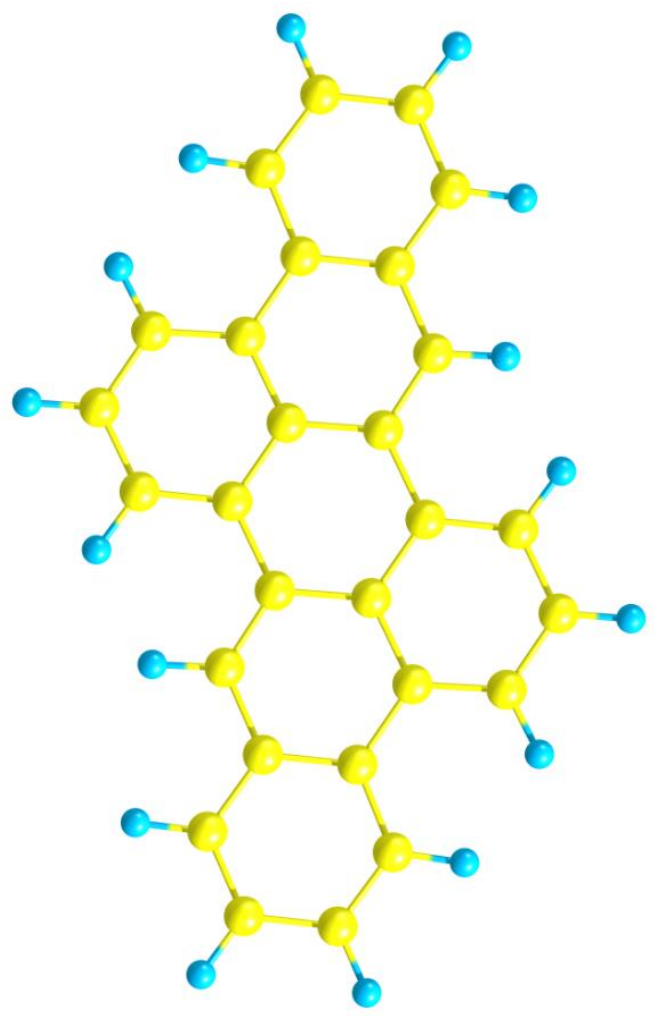




\section{NG3}

56

symmetry c1

\begin{tabular}{|c|c|c|c|}
\hline $\boldsymbol{\tau}$ & 6.130626000 & 0.897358000 & 0.256116000 \\
\hline & 4.866637000 & 1.503473000 & 0.234703000 \\
\hline & 4.796166000 & 2.581934000 & 0.339388000 \\
\hline & 3.696004000 & 0.761402000 & 0.077085000 \\
\hline & 5.018796000 & -1.267563000 & -0.044942000 \\
\hline & 5.114186000 & -2.341282000 & -0.147811000 \\
\hline & 3.765573000 & -0.665867000 & -0.061915000 \\
\hline & 2.512163000 & -1.410061000 & -0.195942000 \\
\hline & 1.266092000 & -0.715467000 & -0.186257000 \\
\hline & 2.503011000 & -2.808472000 & -0.326581000 \\
\hline & 0.046252000 & -1.443817000 & -0.238481000 \\
\hline & 1.313749000 & -3.505900000 & -0.439250000 \\
\hline$C$ & 0.097945000 & -2.831390000 & -0.391306000 \\
\hline I & 3.433650000 & -3.358909000 & -0.348528000 \\
\hline & 1.327299000 & -4.583062000 & -0.561086000 \\
\hline & -3.765573000 & 0.665871000 & -0.061901000 \\
\hline & -3.696006000 & -0.761400000 & 2000 \\
\hline & -5.018801000 & 1.267562000 & -0.044953000 \\
\hline $\mathrm{C}$ & -4.866639000 & -1.503471000 & 0.234713000 \\
\hline $\mathrm{H}$ & -5.114196000 & 2.341277000 & -0.147856000 \\
\hline H & -4.796167000 & -2.581933000 & 0.339393000 \\
\hline & 2.423283000 & 1.408572000 & 0.031572000 \\
\hline $\mathrm{H}$ & 2.429492000 & 2.486892000 & 960000 \\
\hline $\mathrm{C}$ & 1.243524000 & 0.734756000 & -0.124678000 \\
\hline $\mathrm{C}$ & -1.243523000 & -0.734758000 & -0.124657000 \\
\hline $\mathrm{C}$ & -2.423289000 & -1.408573000 & 0.031558000 \\
\hline $\mathrm{H}$ & -2.429507000 & -2.486891000 & 0.124968000 \\
\hline $\mathrm{C}$ & -1.266091000 & 0.715466000 & -0.186250000 \\
\hline $\mathrm{C}$ & -0.046249000 & 1.443813000 & -0.238506000 \\
\hline $\mathrm{C}$ & -2.512157000 & 1.410065000 & -0.195911000 \\
\hline $\mathrm{C}$ & -0.097937000 & 2.831385000 & -0.391347000 \\
\hline $\mathrm{C}$ & -2.502999000 & 2.808481000 & -0.326516000 \\
\hline $\mathrm{C}$ & -1.313739000 & 3.505904000 & -0.439228000 \\
\hline $\mathrm{H}$ & -0.814132000 & -3.404819000 & -0.484609000 \\
\hline $\mathrm{H}$ & 0.814143000 & 3.404800000 & -0.484714000 \\
\hline $\mathrm{H}$ & -3.433634000 & 3.358929000 & -0.348383000 \\
\hline $\mathrm{H}$ & -1.327287000 & 4.583068000 & -0.561048000 \\
\hline $\mathrm{C}$ & -6.206645000 & 0.529145000 & 0.109240000 \\
\hline $\mathrm{C}$ & -6.130629000 & -0.897357000 & 0.256126000 \\
\hline $\mathrm{C}$ & -7.338499000 & -1.637870000 & 0.414250000 \\
\hline $\mathrm{C}$ & -8.553282000 & -1.004047000 & 0.426737000 \\
\hline $\mathrm{C}$ & -8.629335000 & 0.407495000 & 0.281431000 \\
\hline $\mathrm{C}$ & -7.488073000 & 1.151233000 & 0.127323000 \\
\hline $\mathrm{H}$ & -7.546208000 & 2.229168000 & 0.016573000 \\
\hline $\mathrm{H}$ & -9.598220000 & 0.893751000 & 0.293258000 \\
\hline $\mathbf{H}$ & -9.465252000 & -1.577718000 & 0.547765000 \\
\hline $\mathbf{H}$ & -7.278727000 & -2.715608000 & 0.524962000 \\
\hline & 6.206640000 & -0.529147000 & 0.109252000 \\
\hline & 7.488066000 & -1.151238000 & 0.127357000 \\
\hline
\end{tabular}

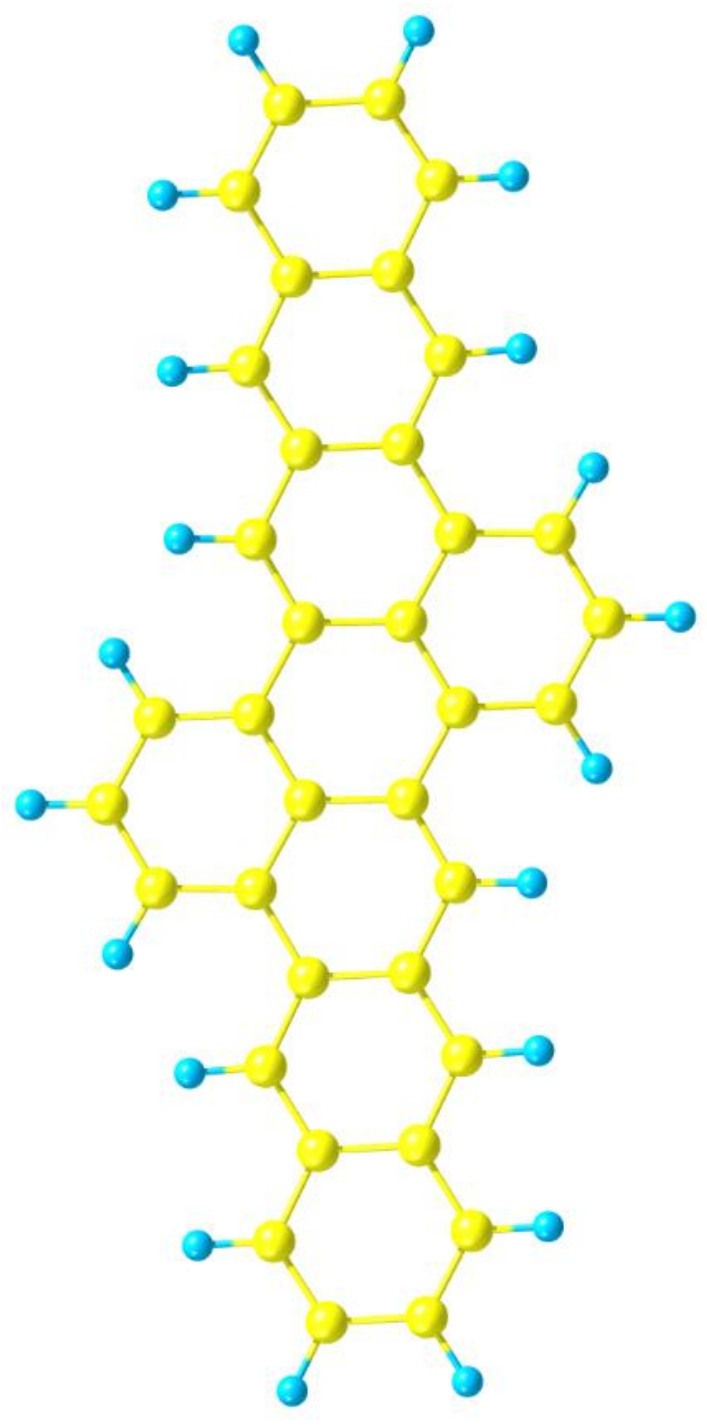




$\begin{array}{lrrr}\mathrm{C} & 8.629330000 & -0.407500000 & 0.281460000 \\ \mathrm{C} & 8.553278000 & 1.004046000 & 0.426736000 \\ \mathrm{C} & 7.338497000 & 1.637872000 & 0.414228000 \\ \mathrm{H} & 7.278727000 & 2.715613000 & 0.524916000 \\ \mathrm{H} & 9.465250000 & 1.577717000 & 0.547756000 \\ \mathrm{H} & 9.598214000 & -0.893758000 & 0.293311000 \\ \mathrm{H} & 7.546199000 & -2.229176000 & 0.016632000\end{array}$




\section{NG4}

44

symmetry c1

$\begin{array}{lrrr}\mathrm{C} & -4.943900000 & -2.906326000 & 0.251128000 \\ \mathrm{C} & -3.568405000 & -2.857744000 & 0.292453000 \\ \mathrm{C} & -5.675252000 & -1.718112000 & 0.070004000 \\ \mathrm{H} & -2.991378000 & -3.766691000 & 0.427716000 \\ \mathrm{H} & -6.758313000 & -1.750445000 & 0.034842000 \\ \mathrm{C} & -2.877882000 & -1.628816000 & 0.155114000 \\ \mathrm{C} & -5.022093000 & -0.508379000 & -0.061037000 \\ \mathrm{H} & -5.616153000 & 0.385853000 & -0.195390000 \\ \mathrm{C} & -3.612193000 & -0.424872000 & -0.018937000 \\ \mathrm{H} & -5.462096000 & -3.852715000 & 0.355776000 \\ \mathrm{C} & -2.878623000 & 0.826547000 & -0.124563000 \\ \mathrm{C} & -1.451966000 & 0.822241000 & -0.062226000 \\ \mathrm{C} & -3.543523000 & 2.059980000 & -0.278752000 \\ \mathrm{C} & -0.734227000 & 2.053260000 & -0.083260000 \\ \mathrm{C} & -2.836351000 & 3.241430000 & -0.358400000 \\ \mathrm{C} & -1.444074000 & 3.239113000 & -0.254872000 \\ \mathrm{H} & -4.622660000 & 2.091354000 & -0.340333000 \\ \mathrm{H} & -3.362272000 & 4.179731000 & -0.493876000 \\ \mathrm{C} & 3.612187000 & -0.424884000 & 0.018916000 \\ \mathrm{C} & 2.877865000 & -1.628829000 & -0.155061000 \\ \mathrm{C} & 5.022091000 & -0.508384000 & 0.060909000 \\ \mathrm{C} & 3.568383000 & -2.857761000 & -0.292413000 \\ \mathrm{C} & 5.675243000 & -1.718119000 & -0.070140000 \\ \mathrm{C} & 4.943880000 & -2.906341000 & -0.251169000 \\ \mathrm{H} & 5.616158000 & 0.385857000 & 0.195172000 \\ \mathrm{H} & 2.991350000 & -3.766713000 & -0.427611000 \\ \mathrm{H} & 6.758306000 & -1.750450000 & -0.035057000 \\ \mathrm{H} & 5.462070000 & -3.852734000 & -0.355812000 \\ \mathrm{C} & -1.455781000 & -1.590505000 & 0.172431000 \\ \mathrm{H} & -0.945009000 & -2.536627000 & 0.300998000 \\ \mathrm{C} & -0.737602000 & -0.430261000 & 0.031987000 \\ \mathrm{C} & 0.737600000 & -0.430271000 & -0.031941000 \\ \mathrm{C} & 1.455765000 & -1.590530000 & -0.172290000 \\ \mathrm{H} & 0.944988000 & -2.536641000 & -0.300903000 \\ \mathrm{C} & 1.451974000 & 0.822231000 & 0.062274000 \\ \mathrm{C} & 0.734236000 & 2.053254000 & 0.083311000 \\ \mathrm{C} & 2.878627000 & 0.826533000 & 0.124585000 \\ \mathrm{C} & 1.444099000 & 3.239106000 & 0.254866000 \\ \mathrm{C} & 3.543540000 & 2.059967000 & 0.278757000 \\ \mathrm{H} & 2.836379000 & 3.241418000 & 0.358383000 \\ & -0.923293000 & 4.184742000 & -0.316594000 \\ \mathrm{H} & 0.923334000 & 4.184746000 & 0.316546000 \\ & 3.362306000 & 4.179720000 & 0.493838000\end{array}$

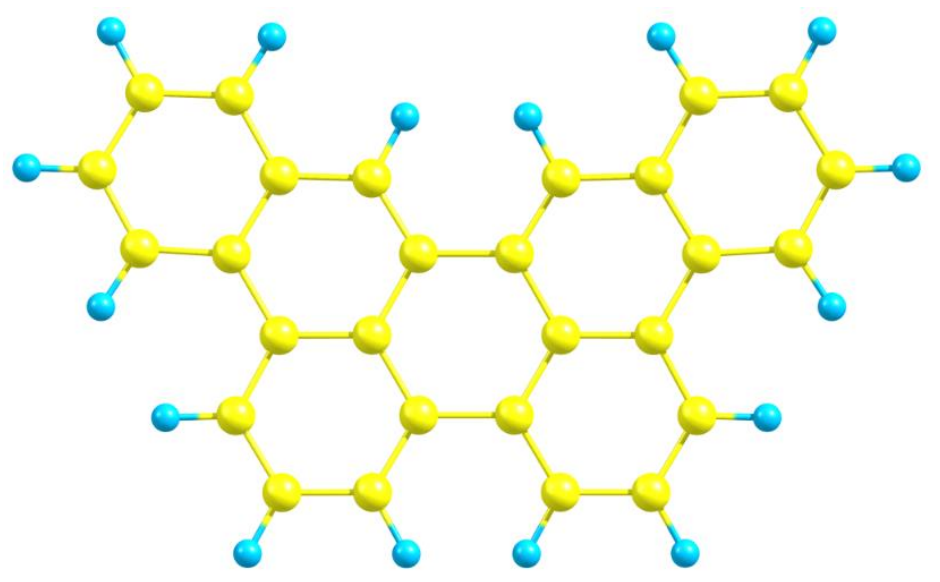




\section{NG5}

46

symmetry c 1

$\begin{array}{lrrr}\mathrm{C} & 6.151535000 & 1.582386000 & 0.000702000 \\ \mathrm{C} & 4.775641000 & 1.671643000 & 0.000610000 \\ \mathrm{C} & 6.785029000 & 0.322996000 & 0.000429000 \\ \mathrm{H} & 4.321773000 & 2.653978000 & 0.000931000 \\ \mathrm{H} & 7.867291000 & 0.260647000 & 0.000418000 \\ \mathrm{C} & 3.958943000 & 0.515759000 & 0.000218000 \\ \mathrm{C} & 6.023441000 & -0.821296000 & 0.000168000 \\ \mathrm{H} & 6.498765000 & -1.796657000 & -0.000010000 \\ \mathrm{C} & 4.605355000 & -0.759035000 & 0.000087000 \\ \mathrm{H} & 6.749341000 & 2.486770000 & 0.001037000 \\ \mathrm{C} & 3.828680000 & -1.945356000 & -0.000118000 \\ \mathrm{C} & 2.449913000 & -1.914752000 & -0.000162000 \\ \mathrm{C} & 1.661709000 & -3.123844000 & -0.000459000 \\ \mathrm{C} & -2.509704000 & -0.569018000 & -0.000114000 \\ \mathrm{C} & -1.769129000 & 0.645140000 & -0.000242000 \\ \mathrm{C} & -4.605378000 & 0.759040000 & 0.000081000 \\ \mathrm{C} & -3.828662000 & 1.945381000 & -0.000126000 \\ \mathrm{H} & -4.341441000 & 2.902209000 & -0.000163000 \\ \mathrm{C} & 2.509730000 & 0.569054000 & 0.000014000 \\ \mathrm{C} & 1.769146000 & -0.645131000 & -0.000112000 \\ \mathrm{C} & 0.347256000 & -0.616301000 & -0.000280000 \\ \mathrm{C} & -0.407116000 & -1.836060000 & -0.000349000 \\ \mathrm{C} & 0.308919000 & -3.088186000 & -0.000467000 \\ \mathrm{C} & -1.791936000 & -1.785807000 & -0.000155000 \\ \mathrm{C} & 1.791903000 & 1.785803000 & -0.000137000 \\ \mathrm{H} & 2.327653000 & 2.726580000 & -0.000280000 \\ \mathrm{C} & -0.347278000 & 0.616291000 & -0.000285000 \\ \mathrm{C} & 0.407087000 & 1.836061000 & -0.000290000 \\ \mathrm{C} & -0.308907000 & 3.088191000 & -0.000511000 \\ \mathrm{H} & 0.265626000 & 4.008589000 & -0.000726000 \\ \mathrm{C} & -1.661735000 & 3.123884000 & -0.000533000 \\ \mathrm{C} & -2.449923000 & 1.914733000 & -0.000311000 \\ \mathrm{C} & -3.958925000 & -0.515753000 & 0.000089000 \\ \mathrm{C} & -4.775607000 & -1.671633000 & 0.000402000 \\ \mathrm{C} & -6.151522000 & -1.582396000 & 0.000687000 \\ \mathrm{C} & -6.785029000 & -0.323050000 & 0.000640000 \\ \mathrm{C} & -6.023440000 & 0.821271000 & 0.000317000 \\ \mathrm{H} & -6.498833000 & 1.796594000 & 0.000236000 \\ \mathrm{H} & -2.186384000 & 4.073565000 & -0.000815000 \\ & -4.321740000 & -2.653975000 & 0.000389000 \\ \mathrm{H} & -6.749254000 & -2.486838000 & 0.000978000 \\ \mathrm{H} & -2.367290000 & -0.260641000 & 0.000824000 \\ \mathrm{H} & -2.1853780000 & -4.008616000 & -0.000432000 \\ \mathrm{H} & -4.073551000 & -0.000721000 \\ \mathrm{H} & -0.000259000\end{array}$

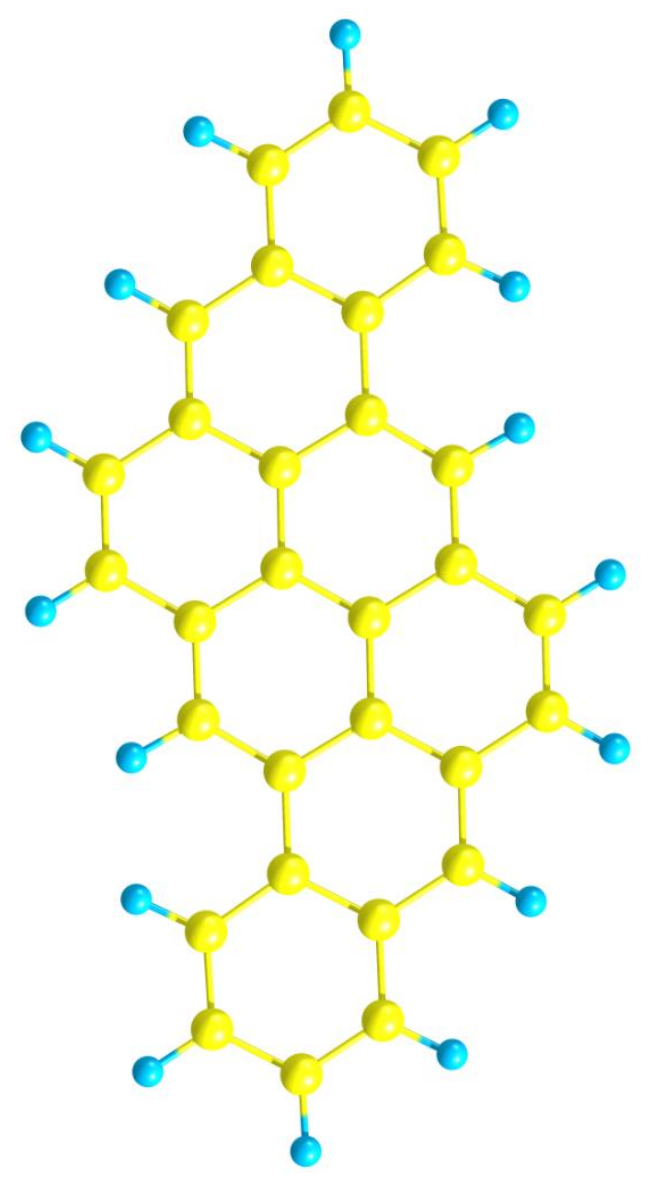




\section{NG6}

58

symmetry c $2 \mathrm{~h}$

\begin{tabular}{|c|c|c|c|}
\hline 工 & -0.748344000 & 6.327570000 & 0.000000000 \\
\hline & -1.000971000 & 4.946587000 & 0.000000000 \\
\hline & -2.037352000 & 4.632143000 & 0.000000000 \\
\hline & 0.020390000 & 3.998974000 & 0.000000000 \\
\hline & 1.641922000 & 5.845817000 & 0.000000000 \\
\hline & 2.673089000 & 6.185740000 & 0.000000000 \\
\hline & 1.385264000 & 4.470294000 & 0.000000000 \\
\hline & 2.452967000 & 3.529951000 & 0.000000000 \\
\hline & 2.231858000 & 2.172213000 & 0.000000000 \\
\hline & 3.321393000 & 1.225324000 & 0.000000000 \\
\hline & 0.224434000 & -2.561043000 & 0.000000000 \\
\hline & -0.876788000 & -1.667908000 & 0.000000000 \\
\hline $\mathrm{C}$ & -1.385264000 & -4.470294000 & 0.000000000 \\
\hline $\mathrm{C}$ & -2.452967000 & -3.529951000 & 0.000000000 \\
\hline & -3.471573000 & -3.905227000 & 0.000000000 \\
\hline & -0.224434000 & 2.561043000 & 0.000000000 \\
\hline$c$ & 0.876788000 & 8000 & 0.000000000 \\
\hline & 0.657579000 & 0.262204000 & 0.000000000 \\
\hline C & 1.763107000 & -0.648774000 & 0.000000000 \\
\hline $\mathrm{C}$ & 3.101509000 & -0.109674000 & 0.000000000 \\
\hline $\mathrm{C}$ & 1.525149000 & -2.014724000 & 0.000000000 \\
\hline $\mathrm{C}$ & -1.525149000 & 2.014724000 & 0.000000000 \\
\hline $\mathrm{H}$ & -2.385380000 & 92000 & 0.000000000 \\
\hline $\mathrm{C}$ & -0.657579000 & 204000 & 0.000000000 \\
\hline $\mathrm{C}$ & -1.763107000 & 0.648774000 & 0.000000000 \\
\hline $\mathrm{C}$ & -3.101509000 & 0.109674000 & 0.000000000 \\
\hline $\mathrm{H}$ & -3.935118000 & 0.804209000 & 0.000000000 \\
\hline $\mathrm{C}$ & -3.321393000 & -1.225324000 & 0.000000000 \\
\hline $\mathrm{C}$ & -2.231858000 & -2.172213000 & 0.000000000 \\
\hline $\mathrm{C}$ & -0.020390000 & -3.998974000 & 0.000000000 \\
\hline $\mathrm{C}$ & 1.000971000 & -4.946587000 & 0.000000000 \\
\hline $\mathrm{C}$ & 0.748344000 & -6.327570000 & 0.000000000 \\
\hline $\mathrm{C}$ & -1.641922000 & -5.845817000 & 0.000000000 \\
\hline $\mathrm{H}$ & -2.673089000 & -6.185740000 & 0.000000000 \\
\hline $\mathrm{H}$ & -4.333868000 & -1.615218000 & 0.000000000 \\
\hline $\mathrm{H}$ & 2.037352000 & -4.632143000 & 0.000000000 \\
\hline $\mathrm{H}$ & 2.385380000 & -2.672292000 & 0.000000000 \\
\hline $\mathrm{H}$ & 3.935118000 & -0.804209000 & 0.000000000 \\
\hline $\mathrm{H}$ & 4.333868000 & 1.615218000 & 0.000000000 \\
\hline $\mathrm{H}$ & 3.471573000 & 3.905227000 & 0.000000000 \\
\hline $\mathrm{C}$ & -0.612944000 & -6.792603000 & 0.000000000 \\
\hline $\mathrm{C}$ & -0.858833000 & -8.198910000 & 0.000000000 \\
\hline $\mathrm{C}$ & 0.178406000 & -9.091993000 & 0.000000000 \\
\hline $\mathrm{C}$ & 1.525149000 & -8.632465000 & 0.000000000 \\
\hline $\mathrm{C}$ & 1.799993000 & -7.290722000 & 0.000000000 \\
\hline $\mathrm{H}$ & 2.827156000 & -6.940677000 & 0.000000000 \\
\hline & 2.334657000 & -9.353576000 & 0.000000000 \\
\hline & -0.021093000 & -10.157653000 & 0.000000000 \\
\hline & -1.886202000 & -8.547929000 & 0.000000000 \\
\hline
\end{tabular}

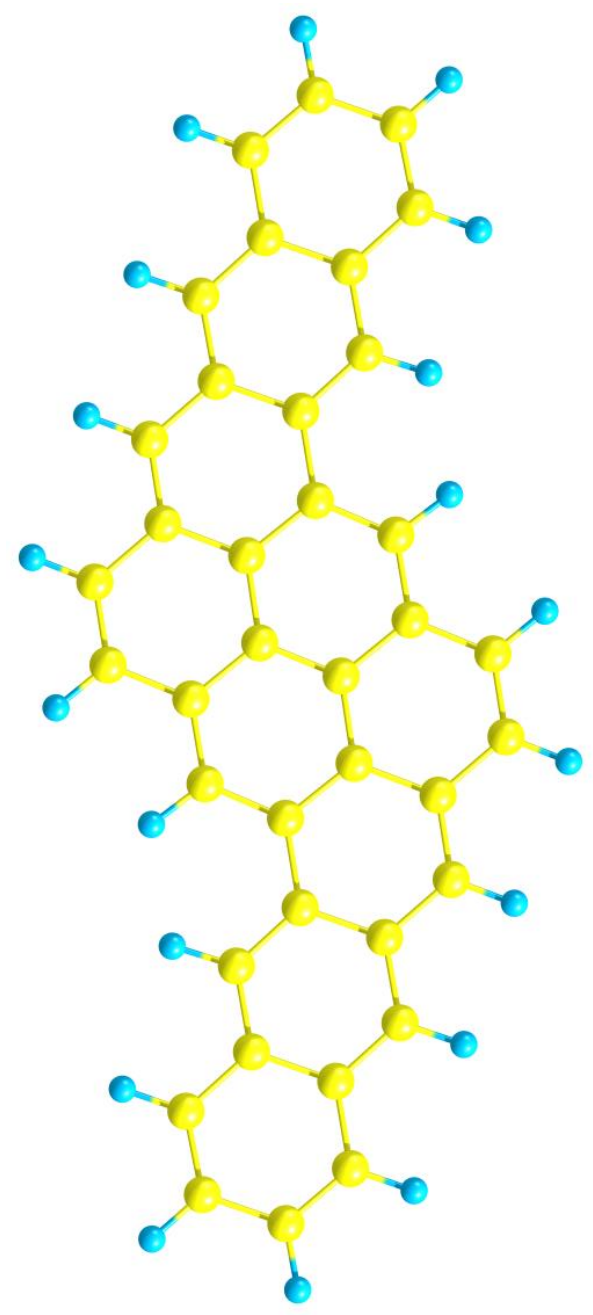




$\begin{array}{lrcc}\mathrm{C} & -1.799993000 & 7.290722000 & 0.000000000 \\ \mathrm{H} & -2.827156000 & 6.940677000 & 0.000000000 \\ \mathrm{H} & -2.334657000 & 9.353576000 & 0.000000000 \\ \mathrm{C} & -1.525149000 & 8.632465000 & 0.000000000 \\ \mathrm{C} & -0.178406000 & 9.091993000 & 0.000000000 \\ \mathrm{H} & 0.021093000 & 10.157653000 & 0.000000000 \\ \mathrm{C} & 0.858833000 & 8.198910000 & 0.000000000 \\ \mathrm{H} & 1.886202000 & 8.547929000 & 0.000000000 \\ \mathrm{C} & 0.612944000 & 6.792603000 & 0.000000000\end{array}$




\section{NG7}

50

symmetry c $2 \mathrm{~h}$

\begin{tabular}{|c|c|c|c|}
\hline & 2.377014000 & -5.039843000 & 0.000000000 \\
\hline & 2.398754000 & -3.642032000 & 0.000000000 \\
\hline & 3.368757000 & -3.161363000 & 0.000000000 \\
\hline & 1.228939000 & -2.870611000 & 0.000000000 \\
\hline & -0.060235000 & -4.972278000 & 0.000000000 \\
\hline & -1.020281000 & -5.479429000 & 0.000000000 \\
\hline & -0.039414000 & -3.567799000 & 0.000000000 \\
\hline$c$ & -1.239369000 & -2.821993000 & 0.000000000 \\
\hline 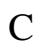 & -1.239369000 & -1.439376000 & 0.000000000 \\
\hline & -2.458923000 & -0.686382000 & 0.000000000 \\
\hline & -1.232680000 & 1.425711000 & 0.000000000 \\
\hline & 0.039414000 & 3.567799000 & 0.000000000 \\
\hline & 1.239369000 & 2.821993000 & 0.000000000 \\
\hline $\mathrm{H}$ & 2.185340000 & 3.354363000 & 0.000000000 \\
\hline$C$ & 1.232680000 & -1.425711000 & 0.000000000 \\
\hline $\mathrm{C}$ & -0.010803000 & 0.718881000 & 0.000000000 \\
\hline & -2.454920000 & 0.671035000 & 0.000000000 \\
\hline $\mathrm{C}$ & 0.010803000 & -0.718881000 & 0.000000000 \\
\hline $\mathrm{C}$ & 2.454920000 & -0.671035000 & 0.000000000 \\
\hline $\mathrm{H}$ & 3.402994000 & -1.191331000 & 0.000000000 \\
\hline $\mathrm{C}$ & 2.458923000 & 0.686382000 & 0.000000000 \\
\hline $\mathrm{C}$ & 1.239369000 & 1.439376000 & 0.000000000 \\
\hline $\mathrm{C}$ & -1.228939000 & 2.870611000 & 0.000000000 \\
\hline $\mathrm{C}$ & -2.398754000 & 3.642032000 & 0.000000000 \\
\hline $\mathrm{C}$ & -2.377014000 & 5.039843000 & 0.000000000 \\
\hline $\mathrm{C}$ & 0.060235000 & 4.972278000 & 0.000000000 \\
\hline $\mathrm{H}$ & 1.020281000 & 5.479429000 & 0.000000000 \\
\hline $\mathrm{H}$ & 3.398213000 & 1.229189000 & 0.000000000 \\
\hline $\mathrm{H}$ & -3.368757000 & 3.161363000 & 0.000000000 \\
\hline $\mathrm{H}$ & -3.402994000 & 1.191331000 & 0.000000000 \\
\hline $\mathrm{H}$ & -3.398213000 & -1.229189000 & 0.000000000 \\
\hline $\mathrm{H}$ & -2.185340000 & -3.354363000 & 0.000000000 \\
\hline $\mathrm{C}$ & -1.109389000 & 5.728517000 & 0.000000000 \\
\hline $\mathrm{C}$ & -1.104445000 & 7.159124000 & 0.000000000 \\
\hline $\mathrm{C}$ & -2.275012000 & 7.863926000 & 0.000000000 \\
\hline $\mathrm{C}$ & -3.528863000 & 7.183755000 & 0.000000000 \\
\hline $\mathrm{C}$ & -3.576428000 & 5.817494000 & 0.000000000 \\
\hline $\mathrm{H}$ & -4.530590000 & 5.300631000 & 0.000000000 \\
\hline $\mathrm{H}$ & -4.446879000 & 7.760433000 & 0.000000000 \\
\hline $\mathrm{H}$ & -2.258077000 & 8.947966000 & 0.000000000 \\
\hline $\mathrm{H}$ & -0.149943000 & 7.675097000 & 0.000000000 \\
\hline $\mathrm{C}$ & 3.576428000 & -5.817494000 & 0.000000000 \\
\hline $\mathrm{H}$ & 4.530590000 & -5.300631000 & 0.000000000 \\
\hline $\mathrm{H}$ & 4.446879000 & -7.760433000 & 0.000000000 \\
\hline $\mathrm{C}$ & 3.528863000 & -7.183755000 & 0.000000000 \\
\hline $\mathrm{C}$ & 2.275012000 & -7.863926000 & 0.000000000 \\
\hline $\mathrm{H}$ & 2.258077000 & -8.947966000 & 0.000000000 \\
\hline $\mathrm{C}$ & 1.104445000 & -7.159124000 & 0.000000000 \\
\hline 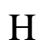 & 0.149943000 & -7.675097000 & 0.000000000 \\
\hline & 1.109389000 & -5.728517000 & 0.000000000 \\
\hline
\end{tabular}

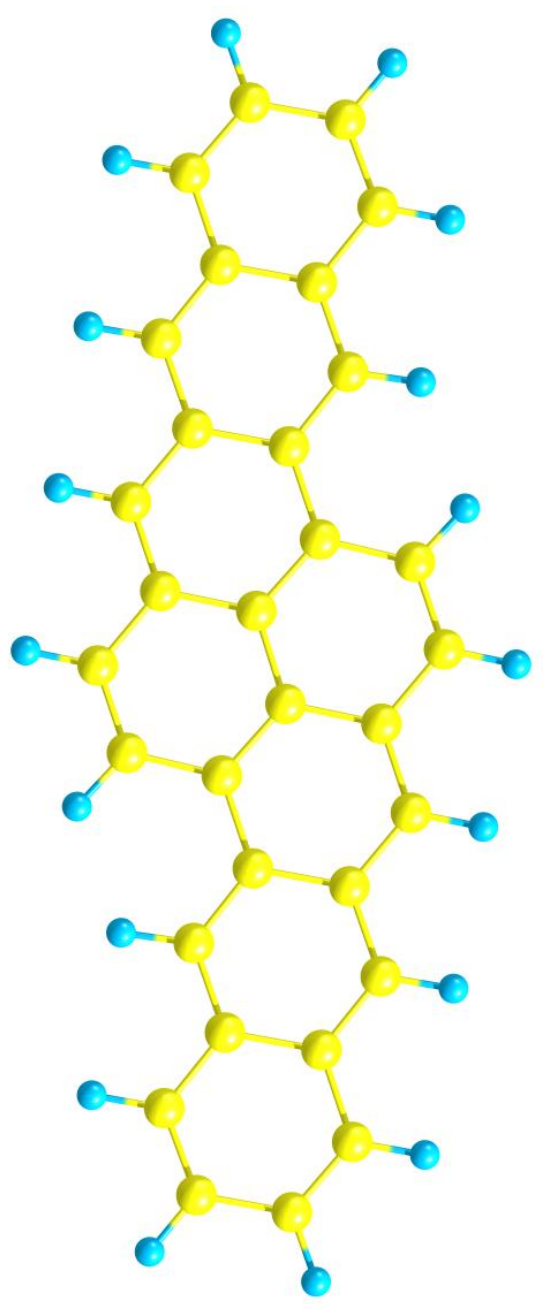




\section{NG8}

44

symmetry cs

$\begin{array}{lrrr}\mathrm{C} & 3.009539000 & 5.902620000 & 0.000000000 \\ \mathrm{C} & 1.906447000 & 5.084749000 & 0.000000000 \\ \mathrm{H} & 0.925466000 & 5.540493000 & 0.000000000 \\ \mathrm{C} & 2.030499000 & 3.666516000 & 0.000000000 \\ \mathrm{C} & 4.479943000 & 3.993803000 & 0.000000000 \\ \mathrm{H} & 5.474068000 & 3.559213000 & 0.000000000 \\ \mathrm{C} & 3.357207000 & 3.115527000 & 0.000000000 \\ \mathrm{C} & 3.530602000 & 1.721679000 & 0.000000000 \\ \mathrm{C} & 2.447701000 & 0.851592000 & 0.000000000 \\ \mathrm{C} & 2.622295000 & -0.568524000 & 0.000000000 \\ \mathrm{C} & 0.208586000 & -0.929558000 & 0.000000000 \\ \mathrm{C} & -2.260093000 & -1.268189000 & 0.000000000 \\ \mathrm{C} & -2.423032000 & 0.137254000 & 0.000000000 \\ \mathrm{H} & -3.428753000 & 0.545682000 & 0.000000000 \\ \mathrm{C} & 0.904226000 & 2.779841000 & 0.000000000 \\ \mathrm{C} & 0.000000000 & 0.466988000 & 0.000000000 \\ \mathrm{C} & 1.557683000 & -1.413857000 & 0.000000000 \\ \mathrm{C} & 1.111092000 & 1.377404000 & 0.000000000 \\ \mathrm{C} & -0.450883000 & 3.265103000 & 0.000000000 \\ \mathrm{H} & -0.634651000 & 4.330822000 & 0.000000000 \\ \mathrm{C} & -1.512939000 & 2.421971000 & 0.000000000 \\ \mathrm{C} & -1.342757000 & 0.996929000 & 0.000000000 \\ \mathrm{C} & -0.926481000 & -1.827227000 & 0.000000000 \\ \mathrm{C} & -0.801861000 & -3.221884000 & 0.000000000 \\ \mathrm{C} & -1.910127000 & -4.075475000 & 0.000000000 \\ \mathrm{C} & -3.372346000 & -2.124588000 & 0.000000000 \\ \mathrm{H} & -4.366518000 & -1.688184000 & 0.000000000 \\ \mathrm{H} & -2.523150000 & 2.817650000 & 0.000000000 \\ \mathrm{H} & 0.178346000 & -3.681457000 & 0.000000000 \\ \mathrm{H} & 1.742724000 & -2.479470000 & 0.000000000 \\ \mathrm{H} & 3.632520000 & -0.964047000 & 0.000000000 \\ \mathrm{H} & 4.537630000 & 1.316351000 & 0.000000000 \\ \mathrm{C} & -3.237108000 & -3.511796000 & 0.000000000 \\ \mathrm{C} & -4.360143000 & -4.397425000 & 0.000000000 \\ \mathrm{C} & -4.183865000 & -5.752694000 & 0.000000000 \\ \mathrm{C} & -2.871712000 & -6.311025000 & 0.000000000 \\ \mathrm{C} & -1.772351000 & -5.497927000 & 0.000000000 \\ \mathrm{H} & -0.773989000 & -5.923158000 & 0.000000000 \\ \mathrm{H} & -2.752161000 & -7.388544000 & 0.000000000 \\ \mathrm{H} & -5.043058000 & -6.413927000 & 0.000000000 \\ & -5.357843000 & -3.970937000 & 0.000000000 \\ \mathrm{C} & 4.315114000 & 5.353200000 & 0.000000000 \\ & 2.880647000 & 6.979108000 & 0.000000000\end{array}$

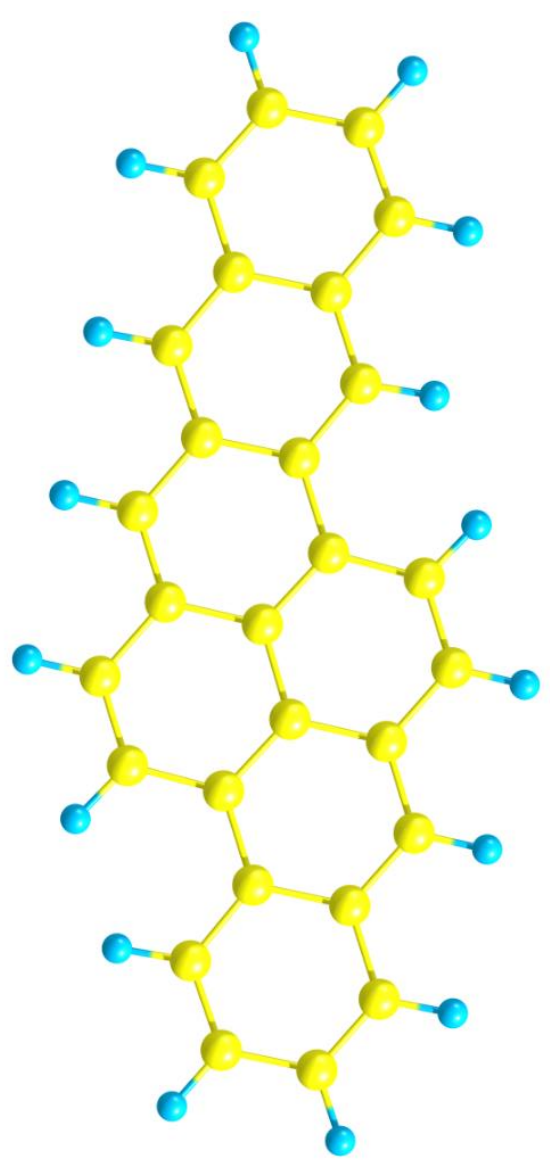




\section{NG9}

64

symmetry c $2 \mathrm{~h}$

\begin{tabular}{|c|c|c|c|}
\hline $\mathrm{C}$ & -0.965834000 & 1.028061000 & 0.000000000 \\
\hline 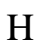 & 2.419070000 & 0.523991000 & 0.000000000 \\
\hline & 1.930918000 & -2.061308000 & 0.000000000 \\
\hline $\mathrm{C}$ & 1.572266000 & -3.395279000 & 0.000000000 \\
\hline 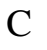 & 2.554904000 & -4.440551000 & 0.000000000 \\
\hline $\mathrm{C}$ & 0.822416000 & -6.164462000 & 0.000000000 \\
\hline $\mathrm{C}$ & -0.952568000 & -7.890987000 & 0.000000000 \\
\hline & -1.919479000 & -6.872419000 & 0.000000000 \\
\hline $\mathrm{H}$ & -2.970420000 & -7.144387000 & 0.000000000 \\
\hline $\mathrm{C}$ & -0.820244000 & -2.768075000 & 0.000000000 \\
\hline $\mathrm{C}$ & -0.176730000 & -5.158965000 & 0.000000000 \\
\hline $\mathrm{C}$ & 2.200790000 & -5.749915000 & 0.000000000 \\
\hline $\mathrm{C}$ & 0.176730000 & -3.766905000 & 0.000000000 \\
\hline $\mathrm{C}$ & -2.192821000 & -3.177562000 & 0.000000000 \\
\hline $\mathrm{H}$ & -2.973689000 & -2.428994000 & 0.000000000 \\
\hline $\mathrm{C}$ & -2.548246000 & -4.490023000 & 0.000000000 \\
\hline $\mathrm{C}$ & -1.564686000 & -5.529076000 & 0.000000000 \\
\hline $\mathrm{C}$ & 0.442629000 & -7.546781000 & 0.000000000 \\
\hline $\mathrm{C}$ & 1.383954000 & -8.614385000 & 0.000000000 \\
\hline $\mathrm{C}$ & 0.981913000 & -9.927509000 & 0.000000000 \\
\hline $\mathrm{C}$ & -1.334428000 & -9.264204000 & 0.000000000 \\
\hline $\mathrm{H}$ & -2.392358000 & 4000 & 0.000000000 \\
\hline $\mathrm{H}$ & -3.595964000 & -4.771444000 & 0.000000000 \\
\hline $\mathrm{H}$ & 2.444070000 & -8.399356000 & 0.000000000 \\
\hline $\mathrm{H}$ & 2.981759000 & -6.497930000 & 0.000000000 \\
\hline $\mathrm{H}$ & 3.602751000 & -4.159327000 & 0.000000000 \\
\hline $\mathrm{H}$ & 2.982981000 & -1.793572000 & 0.000000000 \\
\hline $\mathrm{C}$ & -0.394866000 & -10.260342000 & 0.000000000 \\
\hline $\mathrm{C}$ & 0.438138000 & 1.371234000 & 0.000000000 \\
\hline $\mathrm{C}$ & 0.965834000 & -1.028061000 & 0.000000000 \\
\hline $\mathrm{C}$ & 1.355647000 & 0.321161000 & 0.000000000 \\
\hline $\mathrm{C}$ & -1.355647000 & -0.321161000 & 0.000000000 \\
\hline $\mathrm{H}$ & -2.419070000 & -0.523991000 & 0.000000000 \\
\hline $\mathrm{C}$ & -1.930918000 & 2.061308000 & 0.000000000 \\
\hline $\mathrm{C}$ & -1.572266000 & 3.395279000 & 0.000000000 \\
\hline $\mathrm{C}$ & -2.554904000 & 4.440551000 & 0.000000000 \\
\hline $\mathrm{C}$ & -0.822416000 & 6.164462000 & 0.000000000 \\
\hline $\mathrm{C}$ & 0.952568000 & 7.890987000 & 0.000000000 \\
\hline $\mathrm{C}$ & 1.919479000 & 6.872419000 & 0.000000000 \\
\hline $\mathrm{H}$ & 2.970420000 & 7.144387000 & 0.000000000 \\
\hline $\mathrm{C}$ & 0.820244000 & 2.768075000 & 0.000000000 \\
\hline $\mathrm{C}$ & 0.176730000 & 5.158965000 & 0.000000000 \\
\hline $\mathrm{C}$ & -2.200790000 & 5.749915000 & 0.000000000 \\
\hline $\mathrm{C}$ & -0.176730000 & 3.766905000 & 0.000000000 \\
\hline $\mathrm{C}$ & 2.192821000 & 3.177562000 & 0.000000000 \\
\hline $\mathrm{H}$ & 2.973689000 & 2.428994000 & 0.000000000 \\
\hline $\mathrm{C}$ & 2.548246000 & 4.490023000 & 0.000000000 \\
\hline $\mathrm{C}$ & 1.564686000 & 5.529076000 & 0.000000000 \\
\hline & -0.442629000 & 7.546781000 & 0.000000000 \\
\hline
\end{tabular}

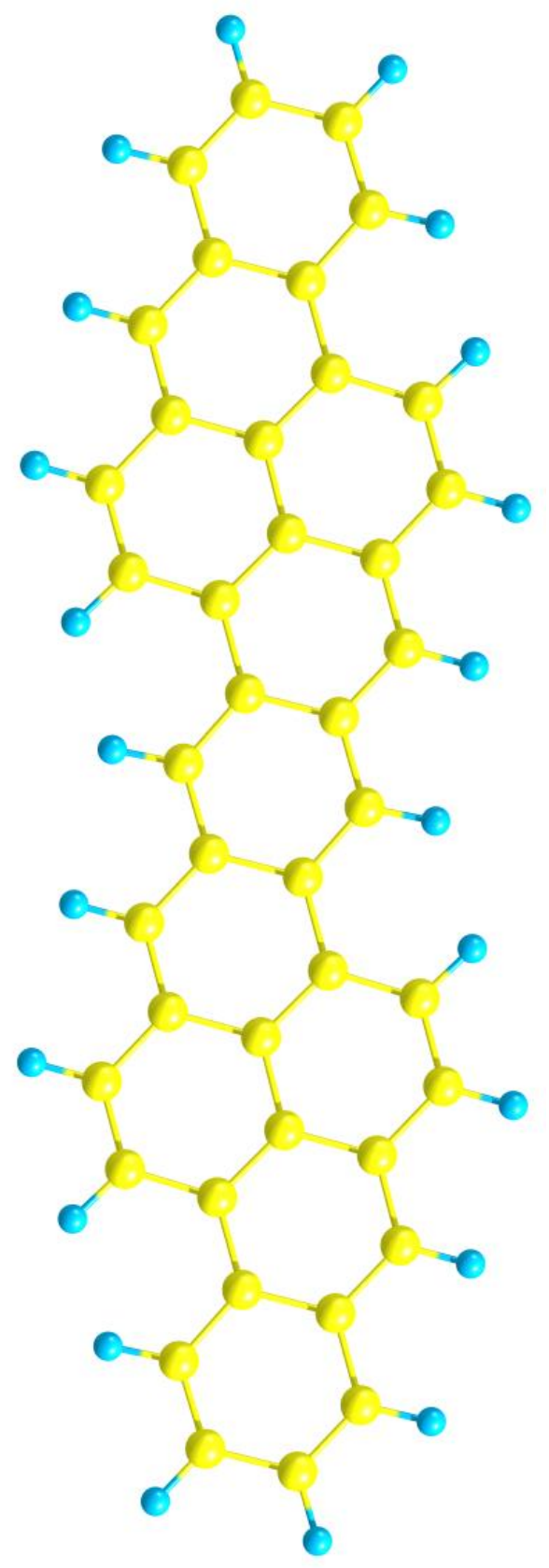




$\begin{array}{lrrr}\mathrm{C} & -1.383954000 & 8.614385000 & 0.000000000 \\ \mathrm{C} & -0.981913000 & 9.927509000 & 0.000000000 \\ \mathrm{C} & 1.334428000 & 9.264204000 & 0.000000000 \\ \mathrm{H} & 2.392358000 & 9.505094000 & 0.000000000 \\ \mathrm{H} & 3.595964000 & 4.771444000 & 0.000000000 \\ \mathrm{H} & -2.444070000 & 8.399356000 & 0.000000000 \\ \mathrm{H} & -2.981759000 & 6.497930000 & 0.000000000 \\ \mathrm{H} & -3.602751000 & 4.159327000 & 0.000000000 \\ \mathrm{H} & -2.982981000 & 1.793572000 & 0.000000000 \\ \mathrm{C} & 0.394866000 & 10.260342000 & 0.000000000 \\ \mathrm{C} & -0.438138000 & -1.371234000 & 0.000000000 \\ \mathrm{H} & 1.724794000 & -10.717114000 & 0.000000000 \\ \mathrm{H} & -0.698916000 & -11.300886000 & 0.000000000 \\ \mathrm{H} & 0.698916000 & 11.300886000 & 0.000000000 \\ \mathrm{H} & -1.724794000 & 10.717114000 & 0.000000000\end{array}$

